Doctoral Thesis

Cerebral bases of cognitive training in working memory executive tasks

Anna Miró Padilla

Supervisor: César Ávila Rivera

October 2018
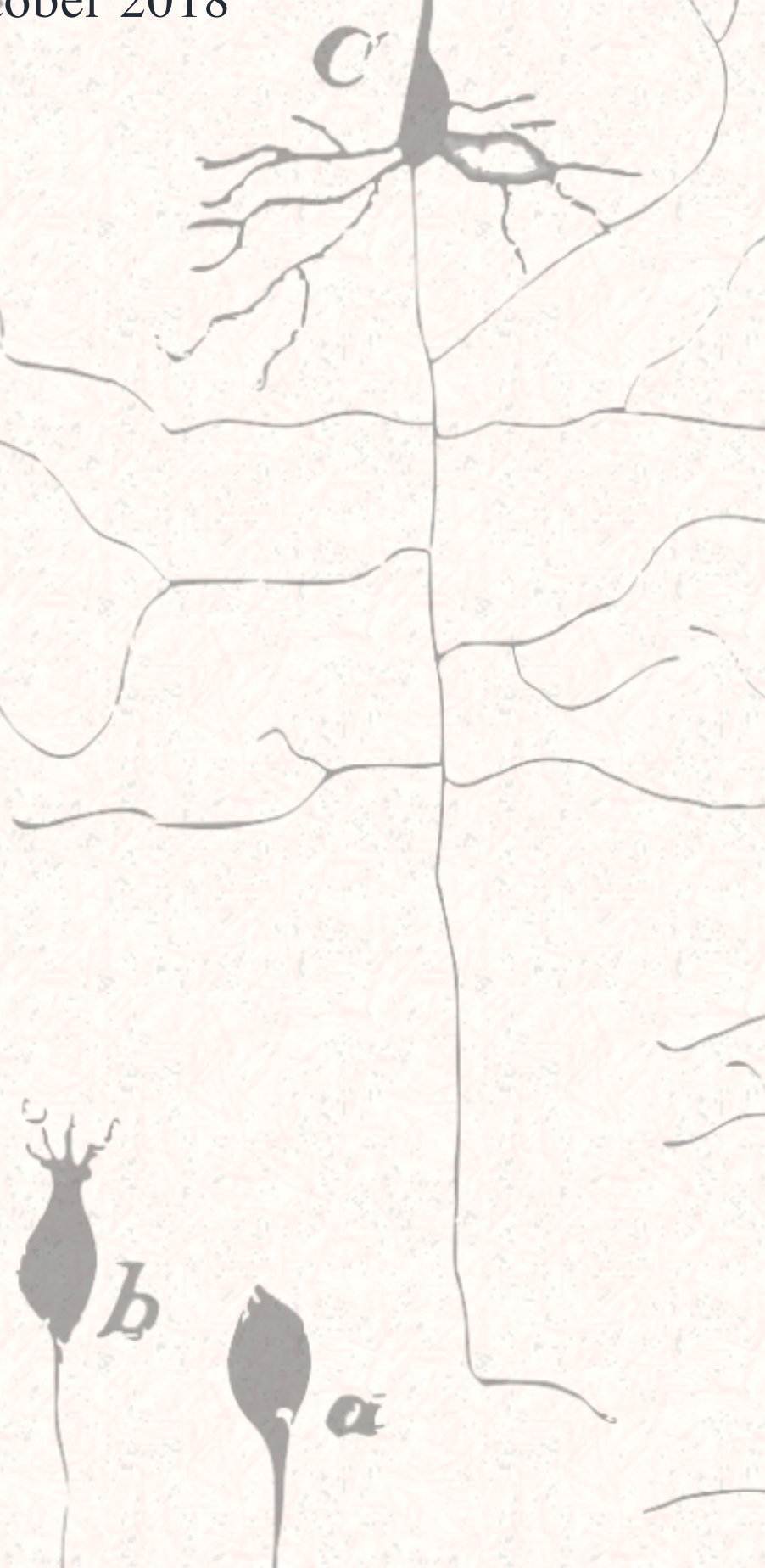


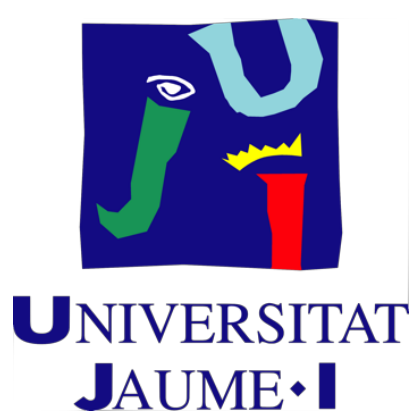

Programa de Doctorat en Psicologia

Escola de Doctorat de la Universitat Jaume I

\section{Cerebral bases of cognitive training in working memory executive tasks}

Memòria presentada per Anna Miró Padilla per a optar al grau de doctora per la Universitat Jaume I

Anna Miró Padilla

César Ávila Rivera

Castelló de la Plana, octubre de 2018 


\section{Finançament rebut}

Aquesta tesi doctoral s'ha pogut realitzar gràcies al finançament de:

Els programes de formació del personal investigador i del personal tècnic i de mobilitat del personal investigador de la Universitat Jaume I de Castelló; ja que la doctoranda ha segut beneficiària de l'ajuda predoctoral per a la formació de personal investigador (FPIUJI) i d'una beca per a realitzar estades temporals en altres centres d'investigació, per al personal docent $\mathrm{i}$ investigador de la Universitat.

Els projectes P1·1B2013-63 de la Universitat Jaume I i PSI2013-47504-R I PSI201678805-R del Ministerio de Economía y Competitividad. 


\section{Imatge de portada i contraportada:}

Dibuix adaptat de Santiago Ramón y Cajal: A-D, mostrant el desenvolupament filogenètic de cèl-lules nervioses madures en una sèrie de vertebrats: a-e, el desenvolupament ontogenètic de cèl·lules en creixement en un mamífer típic (en ambdós casos només es mostren cèl-lules piramidals del cervell); A, granota; B, llangardaix; C, rata; D, home; a, un neuroblast sense dendrites; b, començant per les dendrites; c, les dendrites més desenvolupades; d, primera aparició de branques col·laterals; e, desenvolupament ulterior de colaterals i dendrites. Wikicommons.

"El buen dibujo, como la buena preparación microscópica, son pedazos de la realidad, documentos científicos que conservan indefinidamente su valor y cuya revisión será siempre provechosa, cualesquiera que sean las interpretaciones a que hayan dado origen." (Cajal, 1899b). 
El camí que ha portat al desenvolupament d'aquesta tesi no ha sigut en solitari, per la qual cosa, m'agradaria donar les gràcies a la gent que m'ha ajudat a arribar fins el final.

En primer lloc, donar les gràcies al meu director de tesi, el Dr. César Ávila. Gràcies César per acceptar-me al teu grup i donar-me l'oportunitat de realitzar aquest treball d'investigació que mai m'havia plantejat realitzar. Encara recorde aquell inesperat correu electrònic que em vas enviar dient-me que volies parlar amb mi "sobre una possible col·laboració", col·laboració que s'ha allargat més de cinc anys. Tot aquest temps m'ha permès aprendre moltíssim i descobrir el fantàstic (encara que poc agraït) món de la investigació. Gràcies per facilitar-me els mitjans necessaris per dur a terme la recerca, pels consells donats i per tot el teu temps dedicat.

En segon lloc, a la Dra. Elisensa Bueichekú. Gracias Eli, sin ti no se cómo habría salido todo este trabajo. Desde el momento en el que llegué al laboratorio y durante todo el proceso de desarrollo de la tesis has estado ahí, aunque estuvieras lejos. ¡Cuántas horas cara al ordenador creando la n-back, haciendo correlaciones, analizando el (maldito) resting...! Pero también cuántos buenos ratos de risas y conversaciones hemos compartido. Gracias por tu paciencia infinita enseñándome y por tus siempre útiles y sinceras críticas que me han permitido aprender y crecer como investigadora. Este ha sido el principio de lo que espero que sean muchos años de investigación conjunta y, sobre todo, de amistad.

Donar infinites gràcies al Dr. Jorge Sepulcre i a la Dra. Laura Ortiz. Gracias Jorge por abrirme las puertas de tu laboratorio en Boston, tratarme como una más desde el primer minuto y permitirme vivir una experiencia difícilmente repetible. Tus enseñanzas y consejos me han permitido crecer tanto en lo profesional como en lo personal. Laura, muchas gracias por compartir conmigo tus conocimientos y técnicas de neuroimagen, por haberme integrado con los compañeros de laboratorio, por tus esfuerzos por hacerme hablar inglés y por haber hecho que las horas trabajando pasaran volando.

D’altra banda, agrair al Dr. Alfonso Barrós, a la Dra. Cristina Forn i a la Dra. Noelia Ventura. Gracias Alfonso por tus inigualables aportaciones, enseñanzas y consejos durante todos estos años y por hacerme reflexionar y ver las cosas desde otra perspectiva. Gràcies Cristina pels teus consells i la teua ajuda quan ho he necessitat i per deixar-me aprendre de la teua visió crítica del món acadèmic i dels teus coneixements en el camp de la neuropsicologia clínica. Noelia, gracias por guiarme en mis primeros pasos 
en el mundo de la neuroimagen, por tu ayuda y por estar siempre ahí, dispuesta a contestar todas mis preguntas.

A la resta de companys de laboratori: Jesús, Marian, Maya, Patri, Paola, Mireia, Víctor, Naiara, Lidón i Esteban. Gracias Jesús por las horas de charlas y risas, por tu amistad, tu apoyo y por toda la ayuda que me has prestado sin pestañear desde que llegaste. Marian y Maya, gracias por los buenos momentos compartidos en el laboratorio, por el apoyo y la ayuda que me habéis dado desde que comencé este camino. Es un placer trabajar con vosotras a diario. Paola i Patri, gràcies pel temps compartit, pel vostre suport, ajuda i amistat dins i fora del laboratori. Moltes gràcies Mireia per haver compartit els teus coneixements amb mi, les vesprades a l'escàner amb tu se'm feien més curtes. Gracias Víctor por estar siempre dispuesto a ayudarme. Y gracias Naiara, Lidón y Esteban, llegáis por detrás con una fuerza e ilusión que contagia.

Per a finalitzar, donar les gràcies a la meua família i amics. Gràcies Papà, Mamà i Núria per creure cegament en mi i donar-me el vostre suport incondicional. Gràcies Papà i Mamà per mantindrem els peus a terra, per ensenyar-me el valor de l'esforç i del treball, a ser crítica i a lluitar pel que una vol. Gracias Núria por estar ahí siempre, apoyándome y acompañándome a cada paso. Eres mi ejemplo a seguir y he tenido la gran suerte que seas mi hermana. Gracias Rafa por tu amistad, por tu apoyo todos estos años y por prestarme tu ayuda sin dudar siempre que la he necesitado. Maria, hace muchos años comenzamos un camino juntas que, aunque siga siendo el mismo, nos ha ido separando; gracias por seguir ahí igualmente. Gràcies Causa i Marc per ajudar-me a desemboirarme i fer-me oblidar que estava escrivint una tesi encara que continués davant de l'ordinador. Por último, gracias Alex por estar a mi lado todos estos años. Por apoyarme, comprenderme y ayudarme en cada momento. Gracias por levantarme el ánimo en los peores ratos y por alegrarte por mis logros como el que más. Todavía nos queda un largo camino que recorrer juntos.

Sempre recordaré amb molta estima els bons moments viscuts amb tots vosaltres.

Moltes gràcies a totes i a tots! 
$\begin{array}{ll}\text { List of Abbreviations } & 9\end{array}$

$\begin{array}{lr}\text { List of Figures } & 10\end{array}$

$\begin{array}{ll}\text { List of Tables } & 12\end{array}$

$\begin{array}{ll}\text { JUSTIFICATION } & 13\end{array}$

$\begin{array}{ll}\text { CHAPTER 1. General Introduction } & 17\end{array}$

1. What is working memory? 17

1.1. Working memory background 17

$\begin{array}{ll}\text { 1.2. Short-term memory vs working memory } & 18\end{array}$

2. Models and theories of working memory 20

2.1. Atkinson-Shiffring Model 21

2.2. Baddeley-Hitch Model 23

2.2.1. Phonological Loop 25

2.2.2. Visuospatial Sketchpad 27

2.2.3. Central Executive 28

2.2.4. Episodic Buffer $\quad 29$

2.2.5. The latest version of the model 30

2.2.6. Neuroanatomical localization of the components 31

2.3. Cowan's Embedded Processes Theory 34

2.4. A facet model of working memory 37

2.5. Component processes view of working memory 38

2.6. Conclusion 40

3. Working memory and the brain 41

3.1. Brain areas related to working memory 41

3.1.1. The role of frontal lobe 43

3.1.2. The role of parietal lobe $\quad 47$

3.1.3. The role of subcortical areas $\quad 50$

3.1.4. The role of the temporal lobe 53

3.2. Conclusion $\quad 54$

4. Working memory tasks: $n$-back and PASAT 55

4.1. $N$-back 56

4.2. PASAT 60

5. Brain plasticity, automaticity and cognitive training 62

5.1. Working memory training 66

5.1.1. Brain effects of working memory training on monkeys 68

5.1.2. Brain effects of working memory training on humans 69

5.2. The controversy of working memory training: transfer effects 76

$\begin{array}{ll}\text { 5.3. Conclusion } & 81\end{array}$ 
1. Overview of the experiment 82

2. Objectives and hypothesis $\quad 89$

3. Experimental studies 91

3.1. Study 1: Long-term brain effects of N-back training: an fMRI study 92

3.2. Study 2: Locating neural transfer effects of N-back training in the central $\begin{array}{ll}\text { executive: a longitudinal fMRI study } & 128\end{array}$

3.3. Study 3: Sustained and transient gray matter volume changes after N-back training: a VBM study

CHAPTER 3. General discussion

General conclusions 


\section{LIST OF ABBREVIATIONS}

ACC: anterior cingulate cortex

AC-PC: anterior commissure-posterior commissure plane

AI: anterior insula

ANOVA: analysis of variance

BA: Brodmann area

BOLD: blood-oxygen-level dependent imaging

CRUNCH: compensation-related

utilization of neural circuits hypothesis

DLPFC: dorsolateral prefrontal cortex

dMTS: delayed matching-to-sample

task

DTI: diffusion-tensor imaging

EEG: electroencephalography

FA: fractional anisotropy

fMRI: functional Magnetic Resonance

Imaging

FWE: family-wise error

FWHM: full-width at half-maximum

Gf: fluid intelligence

GM: gray matter

HIV: human immunodeficiency virus

IFG: inferior frontal gyrus
IPC: inferior parietal cortex

IQ: intelligent quotient

MD: mediodorsal nucleus

MNI: Montreal Neurological Institute

MRI: magnetic resonance imaging

MTL: medial temporal lobe

PASAT: paced auditory serial addition

test

PET: positron emission tomography

PHC: parahippocampal cortex

PRC: perirhinal cortex

ROI(s): region(s) of interest

$\mathbf{R T}(\mathbf{s})$ : reaction time(s)

S1, S2, S3: Session 1, 2 and 3

respectively

SFG: superior frontal gyrus

SMA: supplementary motor area

SPC: superior parietal cortex

SPM: Statistical Parametric Mapping

TIV: total intracranial volume

TS: training session

VBM: voxel-based morphometry

WAIS-III-R: Wechsler Adult

Intelligence Scale 


\section{LIST OF FIGURES}

\section{CHAPTER 1. General Introduction}

Figure 1: Atkinson-Shriffrin Model of memory (1968).

Figure 2: Baddeley and Hitch working memory system $(1971 ; 2000)$.

Figure 3: Baddeley, Allen and Hitch (2011) revised multi-component model.

Figure 4: Cerebral locations of the different components of the multi-component model.

Figure 5: Cowan's model of the information-processing system (1988).

Figure 6: Working memory processes and interactions in a process-based view.

Figure 7: Working memory main effects across 189 working memory studies.

Figure 8: Regions involved to executive processes of working memory.

Figure 9: Four examples of $n$-back task using different stimuli.

Figure 10: $N$-back main effects across 24 fMRI studies using $n$-back.

Figure 11: Example of the PASAT task.

Figure 12: Brain activation during the performance of PASAT in healthy controls.

Figure 13: Elements underlying working memory training-related increases in capacity.

Figure 14: Changes in gray matter volume after working memory training.

\section{CHAPTER 2. Experimental Section}

\section{Study 1}

Figure 1: Schematic description of the experimental design.

Figure 2: Schematic example of the three conditions of our $n$-back fMRI task and block timing details of the $n$-back fMRI task.

Figure 3: Results of the $n$-back accuracy behavioral analysis and results of the $n$-back RTs behavioral analysis. 
Figure 4: Results of the behavioral $n$-back training analysis of training group.

Figure 5: 2-back and 3-back general task activations in Session 1.

Figure 6: Results of the adaptive $n$-back post-training effects for 2-back load level.

Figure 7: Results of the adaptive $n$-back post-training effects for 3-back load level.

Supplementary Figure: One Sample $t$ test for the control group in (a) Session 1, (b)

Session 2, and (c) Session 3, showing the $n$-back general activations.

\section{Study 2}

Figure 1: Results of the PASAT behavioral analysis.

Figure 2: PASAT general task activations in Session 1.

Figure 3: Results of the adaptive $n$-back post-training effects for PASAT.

Figure 4: Correlations between the improvement in PASAT scores and left DLPFC.

Figure 5: Overlap of the $n$-back and PASAT activations.

\section{Study 3}

Figure 1: Schematic description of the experimental design.

Figure 2: Brain areas with GM volume changes in the trained group after perform a specific brief $n$-back training and GM volume (in ml) evolution across sessions in both groups in right superior parietal cortex and in right putamen.

Figure 3: Right putamen and right superior parietal cortex GM volume change (in $\mathrm{ml}$ ) from S1 to S2 relationships with the Matrix Reasoning Test direct scores. 


\section{LIST OF TABLES}

\section{CHAPTER 1. General Introduction}

Table 1: Differences between short-term memory and working memory.

\section{CHAPTER 2. Experimental Section}

\section{Study 1}

Table 1: List of brain activations in 2-back load level between groups comparison.

Table 2: List of brain activations in 3-back load level between groups comparison.

\section{Study 2}

Table 1: List of brain activations in PASAT between groups comparison. 
Imagine that you and your friends have just visited an important museum where different art styles are on display. One friend starts a conversation by saying that contemporary art is not really an "art", and he begins to make his case. You disagree and want to explain your arguments, but another friend interrupts you and defends contemporary art. She says that contemporary art is in line with our advancing world and the new technologies. You agree with her and think you have better arguments, but you cannot talk because she has not finished yet. In this situation, your brain is doing different things at once: listening to your friend and, at the same time, creating your reasons and the sentences you are going to say, changing them in order to avoid repeating her arguments when you finally speak, and maintaining your own argument until she stops talking. This is your working memory functioning in full swing.

As our example shows, working memory is necessary for a significant range of cognitive processes. It is a critical psychological function in daily life because it is a determinant in comprehension, reasoning, and problem-solving. Working memory refers not only to the capacity to retain information, but also to manipulate and transform it in order to plan and guide our behavior. Therefore, it is necessary to understand how working memory works at the behavioral and brain levels. Knowing how working memory works is a relevant issue not only for basic psychology, but also for applied disciplines such as clinical neuropsychology and neurorehabilitation. 
It has been shown that the brain is capable of reorganizing by modifying its structures and connections in order to adapt to environmental or internal changes. This capacity is called neuroplasticity. In recent years, studies have demonstrated that training in a cognitive process (e.g. semantic memory or fluency) causes a reorganization of the cerebral resources and improves that specific process. It is essential to completely understand how cognitive training contributes to the neuronal reorganization processes. The study of the capacity of cognitive training to modify the brain can be used to benefit learning in the healthy population and in patients with brain damage, dementia, or intellectual disability. Thus, knowledge about the cerebral basis of cognitive training is essential to properly apply neurorehabilitation programs. We believe that understanding how the healthy brain changes due to learning, training, and automation makes it possible to develop better neuropsychological theories and better neurorehabilitation programs that, in the long run, may be implemented.

The use of neuroimaging techniques is crucial to understanding how the brain functions. Functional Magnetic Resonance Imaging (fMRI) allows the non-invasive study of the cerebral plasticity processes that occur in the human brain. This measure has good temporal resolution and excels in the spatial domain, thus being suitable for human studies that involve one or more explorations. fMRI is the most widely used neuroimaging technique in cognitive training studies.

So, can we train working memory to improve it? A large amount of research has been done in recent years to try to answer this question, but without reaching any agreement. Most of the research that has been carried out on working memory has focused on behavioral studies. Therefore, there is a need to perform working memory training studies 
to understand the brain changes that cognitive training causes, and these data have to be longitudinal to figure out whether the consequences of the training remain over time after finishing it.

In our research group at the Universitat Jaume I, Neuropsychology and Functional Neuroimaging group, several studies have been carried out in the past few years about brain changes related to training on a task and the effects of practicing a skill. Hence, the studies have demonstrated that visual search training produces reduced activation in the posterior parietal cortex, and the observed training-related decreases could be associated with increased neural efficiency in specific key regions for task performance (Bueichekú et al., 2016). Moreover, Resting-State Functional Connectivity demonstrated that by studying the brain changes it is possible to predict the learning of foreign sounds (Ventura-Campos et al., 2013) and visual search efficiency (Bueichekú et al., 2015). The present study follows along the lines of the aforementioned studies and focuses on the neural basis of working memory training and investigating whether training in one specific task can be useful to perform another non-trained task. This project has the novelty of studying neural changes in the long-term in order to find out whether the effects of cognitive training remain stable, disappear, or change over time. We wanted to add an important piece of information to the current literature because, to date, we have not found any studies that include a follow-up session to evaluate the stability of the longterm effects in the brain after working memory training in healthy adults.

The central purpose of this thesis is to investigate the cerebral changes associated with working memory training and contribute more empirical data to the theories on the development of neurobehavioral and automaticity working memory. To this end, a 
longitudinal fMRI working memory paradigm will be used. We will explore the behavioral, functional and anatomical data before a brief working memory training, immediately after it, and five weeks after finishing the training. To do so, different analytical techniques are used, and the results are interpreted according to current neurocomputational theories. 


\section{What is working memory?}

The aforementioned scenario in the justification is only one of many examples of working memory uses. Following any movie's storyline, keeping in mind a phone number before dialing it or an address while you are driving, mentally preparing questions in a class to ask when the professor has finished, understanding a sentence, thinking of various possible strategies to beat your friend in a game, measuring and combining the ingredients without looking at the recipe... would be other valid examples. Every day, we keep pieces of information in mind in situations where there is no other external record, and we perform cognitive operations with them, manipulating or transforming them over short periods of time. This is working memory.

Working memory is a central cognitive process that interacts with other processes such as perception, short-term memory, long-term memory, and goal-directed actions. It is a set of processes that manipulates and transforms information through additional processes to guide decision-making and behavior. It can be defined as "a workspace that provides a temporary holding store so that relevant information is highly accessible and available for inspection and computation. When cognitive tasks are accomplished, the information can be easily erased, and the processes can begin again with other information" (Braver, 2006, p. 240). Although many cognitive functions can be carried out without the need for working memory, it is necessary for crucial cognitive processes, functions, and behaviors such as reading, reasoning, learning, or inhibition. 
Working memory capacity is not unalterable and unlimited. Any distraction could cause us to lose the information that we had been storing in the working memory space. If we lose our attention on what is being held in the working memory, it will be gone, and we will have to start the process of entering information again. The same thing will happen if we try to store too much information. Although people vary in their working memory capacity, it is quite restricted, even in a healthy population. In addition, a difficult cognitive task reduces the amount of space in the working memory and can result in a loss of information already held.

\subsection{Working memory background}

The concept of working memory is not new, but it has evolved during the past hundred years and can be tracked to the early days of modern psychology. William James (1890), an American psychologist, was the first to propose the distinction between two types of memory: primary memory and secondary memory. Primary memory was defined as the initial storage where the information was accessible and could be consciously manipulated. On the other hand, James defined secondary memory similarly to the current concept of long-term memory, which is all the information that we store for a lifetime that cannot be retrieved without initiating an active cognitive process (James, 1890). It was not until the second half of the 20th century when we again began to find studies on the short-term storage system due to the dominance of behaviorist views. A growing need for better anatomic knowledge gave rise to the development of new neuroimaging methods for exploring cognitive problems. After the two world wars, a number of cases of acquired brain injury where patients had long-term memory disorders, but preserved short-term memory (or the opposite), were studied and showed that short and long-term memory did not share the same anatomic surface. In 1949, Donald Hebb proposed two 
separate storage systems, one temporary and other permanent, that were separated in the brain (Hebb, 1949).

An influential cognitive theorist, Gorge Miller, studied the limitations of short-term memory in his famous paper "The Magical Number Seven Plus or Minus Two", which is sometimes referred to as Miller's Law. Based on short-term memorization tests, the author claimed that young adults could retain about seven items of information in shortterm memory. Miller proposed that memory is not limited in terms of single items; they can be grouped into "chunks". A chunk is a group of items that a person organizes. For example, three single numbers $(2,4,8)$ can be regrouped in only one $(248)$. What counts as a chunk depends on the knowledge of the individual, and it can be managed to expand its capacity (Miller, 1956). Years later, Nelson Cowan changed this statement, determining that the storage capacity is much less than seven, specifically $4 \pm 1$ (Cowan, 2001).

The working memory construct evolved from earlier ideas that proposed the existence of different short-term storage (López, 2011). The term working memory was first used in the book by Miller, Galanter and Pribram (1960) to give short-term memory a more practical point of view. They literally wrote “(...) we should like to speak of the memory we use for the execution of our plans as a kind of quick-access, working memory" (Pribram, Miller \& Galanter, 1960, p. 65). From then on, the working memory concept began to be used in different theories and models, and it became famous when it was adopted by Baddeley and Hitch (1974). They finally distinguished short-term memory from working memory in their well-known model, defining working memory as a limited capacity system that provides temporary storage and manipulation of information 
necessary for complex cognitive tasks such as comprehension, learning, and reasoning (Baddeley, 1992).

\subsection{Short-term memory vs. working memory}

Although it is now known that they are different concepts, some authors have been using short-term memory and working memory indistinctly in the literature due to the common aspects they share. Cowan (2009) said that "the distinction between short-term memory and working memory is one that depends on the definition that one accepts" (Cowan, 2009, p. 13). In the work by Aben, Stapert, \& Blokland (2012), the authors explained the complex conflict of terms and definitions that can be found in the current literature, and they concluded that the differentiation between these two kinds of memory is far from clear. We believe that it is important to know the differences between the two concepts. Short-term memory has been considered to have only one component and only hold information passively, whereas working memory has multiple components and actively manipulates and processes information (López, 2011). In his book, Dehn (2008) makes a good comparison of short-term and working memory that we reproduce below in Table 1. 
Table 1: Differences between short-term memory and working memory (Dehn, 2008).

\begin{tabular}{c|c}
\hline Short-term memory & Working memory \\
\hline $\begin{array}{c}\text { Passively holds information } \\
\text { visual) }\end{array}$ & Actively processes information \\
\hline $\begin{array}{c}\text { Its capacity is domain specific (verbal and } \\
\text { Activates information stored in long-term } \\
\text { memory mechanically }\end{array}$ & $\begin{array}{c}\text { Its capacity is less domain specific } \\
\text { from long-term memory }\end{array}$ \\
\hline $\begin{array}{c}\text { No management functions } \\
\text { memory operate regardless of long-term } \\
\text { metains information coming from external } \\
\text { conditions }\end{array}$ & $\begin{array}{c}\text { Some executive functions } \\
\text { Operations depend on long-term } \\
\text { memory structures }\end{array}$ \\
\hline $\begin{array}{c}\text { Rempormation of some cognitive } \\
\text { processes }\end{array}$ \\
\hline $\begin{array}{c}\text { Related to learning and high-level } \\
\text { cognitive functions }\end{array}$ \\
\hline
\end{tabular}

\section{Working Memory Models}

A number of models and theories have been proposed over the past half-century for how working memory works. Below, we outline some of the most relevant ones.

\subsection{Atkinson-Shiffrin Model}

In 1968, Richard Atkinson and Richard Shiffirn proposed a model for how memory works in humans, called the Atkinson-Shriffrin Model (Atkinson \& Shiffrin, 1968). It was also known in the literature as the Multi-Store Model or Modal Model because it assembled many of the characteristics of other models proposed in the 1960s(Goldstein, 2008). This conception asserted that the human memory processes in a linear manner, dividing it into three major components organized according to the duration of the information in each 
of them. The components were three types of storage: a sensory register, a short-term store, and a long-term store (Figure 1).

The sensory register (also sensory memory or sensory buffer) is a very brief form of memory that only lasts for milliseconds. Its function is to detect and hold the information for use in short-memory without processing it. The information is quickly forgotten if it does not draw attention. The data that are attended to are transferred to the short-term memory (Atkinson \& Shiffrin, 1968). There is a sensory register for each sense, but this form of memory has been more associated with the visual (called iconic memory) and auditory (called echoic memory) perception processes. The elements of the sensory register are collected only by external stimulation, in contrast to the other two types of memory described by this model. The short-term store (also short-term memory) described by Atkinson and Shiffrin is a form of memory with limited capacity (5-7 items) and duration (15-30 seconds). The duration will depend on the modality. The information input to short-term memory will last only a few seconds unless it is maintained through a process called rehearsal. This process works by repeating the data over and over and allows information to be stored in the long-term memory permanently. Therefore, whether the information codifies and arrives to the long-term memory depends on the short-term memory. The stored information does not have to be the same modality as its sensory input; for example, auditory input can be attended to as visual data. The amount of information that can be held and attended to in this storage is limited. Finally, the last component of the Model is the long-term store (or long-term memory). This is a permanent storage of information that can be held for years. These data can be transferred to the short-term memory in order to work with it and manipulate it. Following the Model, the long-term memory is limitless in its duration and capacity. 
Figure 1: Atkinson-Shriffrin Model of memory (1968).

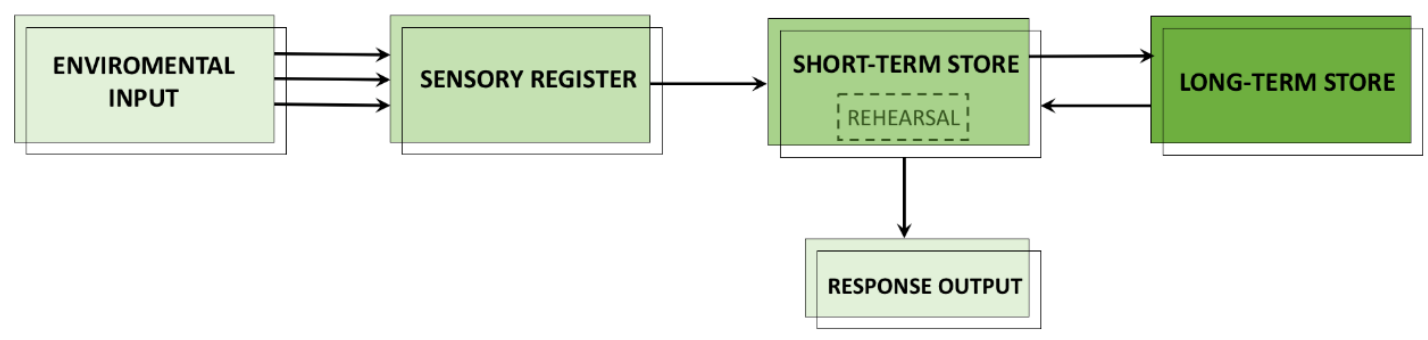

The Atkinson-Shriffrin Model of memory (1968) has a temporal sequential relationship between the different memories. Following this assumption, a disruption in the shortterm store would have consequences at the same time in the long-term store. Today, we know that this is not true. Clinical studies have demonstrated that an alteration in the short-term memory does not mean that the long-term memory will be affected too. This evidence led experts to consider that the memory components would not be linear, but rather independent parts whose association may work in parallel (López, 2011). In addition, the emergence of new working memory theories resulted in the disuse of this Model. In any case, the Multi-Store Model was extremely influential because it provided an easy explanation of information processing in memory, it stimulated research on shortterm memory, and it was a useful framework for interpreting memory performance (Braver, 2006).

\subsection{Baddeley-Hitch Model}

According to the Modal Model, it is not possible to carry out two tasks simultaneously because one of these tasks occupies the entire short-term memory. Baddeley and Hitch (1974), demonstrated in a number of experiments that humans were able to perform two tasks at the same time, such as reading or reasoning and remembering numbers at a time. These experiments led to the conclusion that short-term memory was a dynamic process with different components that can work individually. Baddeley and Hitch (1974) 
proposed a model where short-term memory came to be called working memory, defining it as "a system for the temporary holding and manipulation of information during the performance of a range of cognitive task such as comprehension, learning, and reasoning" (Baddeley, 1986, p. 34). They proposed a system that involves three main components: two short-term specialized temporary stores and one control system. The Baddeley-Hitch model (also called the multi-component model) changed the concept from one lineal, structural, and temporal vision of short-term memory to one functional and operational vision that maintains and manipulates the necessary information to perform different cognitive tasks. We are dealing with a functional system, which gives us the ability to manipulate and update the information in memory to achieve our task goals. Initially, the multi-component model consisted of three components: the phonological loop, the visuospatial sketchpad, and the central executive (Figure $\mathbf{2 A}$ ). A fourth component of the model was proposed by Baddeley (2000) in order to explain how the segregated information is integrated into a global experience and how it is related to long-term memory: the episodic buffer (Figure 2B).

A

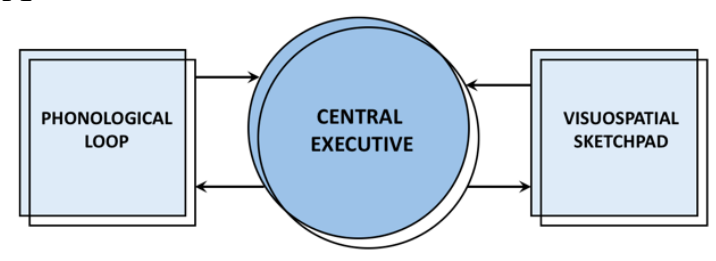

Figure 2: (A) Diagram of the initial three-component model of working memory proposed by Baddeley and Hitch (1971).

(B) Diagram of Baddeley's revised working memory system (Baddeley, 2000).

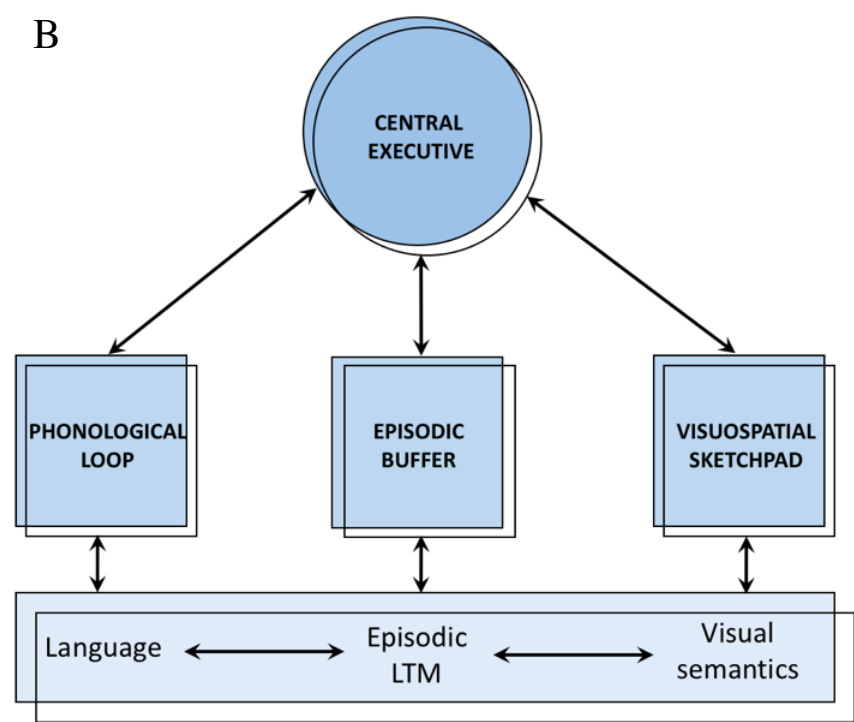




\subsubsection{Phonological Loop}

The Phonological loop is the best developed component of the working memory model (Baddeley, 2000). It was postulated to explain the evidence that existed about language codification in the long-term memory. It is in charge of preserving the language information containing verbal and auditory data. Information can enter into the phonological loop, presented auditory or visually, and it can come from external input as well as from inside our cognitive system. Reading information is transformed by silent articulation into the phonological code. The phonological loop involves two subcomponents: the phonological store and the articulatory rehearsal process. The first subcomponent is a temporary input store that has a limited capacity and holds speech sounds for a short period of time ( 2 seconds or less). The second subcomponent is in charge of avoiding the decay of information in the phonological store by means of repetition of the speech elements on a loop. The articulatory rehearsal process is also responsible for transforming the visually presented language into phonological code. Importantly, data in the phonological store will disappear unless the articulatory rehearsal process takes action to indefinitely maintain the information. Both subcomponents are supported by neuropsychological evidence. Patients with aphasia with developmental verbal dyspraxia are unable to set up the speech motor codes necessary for articulation, caused by a deficiency in the articulatory rehearsal process (Waters, 1992). Patients with dysarthria show a normal capacity for rehearsal, suggesting that it is the subvocal rehearsing that is crucial (Baddeley \& Wilson, 1985). Moreover, patients with brain damage who had language processing abilities intact were not able to perform verbal working memory tasks correctly, especially with auditory information. These patients had a common pattern of deficits and brain damage in the left inferior parietal cortex, indicating that their phonological loop was damaged (Vallar \& Papagno, 
2002). At the same time, behavioral studies suggest a number of phenomena that provide evidence for the phonological loop, and they are discussed in detail below:

- Phonological Similarity Effect: The letters or words with similar sounds (for example, $V, B, G, T$ and sun, son, or some) are more difficult for people with normal phonological processing to remember than words that are phonologically different. This effect supports Baddeley's assumption that verbal information is encoded phonetically in working memory, and it has been demonstrated in a large number of studies (Baddeley, 2003a).

- Word Length Effect: Short words (such as song, ice, fire, time...) are easier to remember than the same number of long words (e.g., ocarina, adventure, atmosphere, disability...). It takes longer to rehearse long words than short ones. The important thing in this effect is not the number of the syllables, but the time it takes to pronounce the word. The speaking time affects the speed of silent rehearsal, and so it would be easier to forget words that take longer to pronounce. According to Baddeley, the number of words you can pronounce in two seconds should be your verbal memory span.

- Articulatory Suppression: This effect occurs when people are disrupted while they perform a verbal working memory operation, keeping them from rehearsing the information. The efficiency is significantly impaired. Participants have to try to maintain visually presented words in working memory while they have to repeat aloud an irrelevant sound (for example "the, the, the..."). Speaking interferes with phonological processing and rehearsal and allows researchers to assess pure phonological loop capacity. Repeating a sound voids the word length effect, producing the loss of both long and short words from the phonological store.

This component acts as support for a wide range of verbal working memory tasks. Importantly, it is related to language comprehension and processing and plays a crucial role in language acquisition. Furthermore, this working memory component evolves to facilitate language acquisition (Baddeley, Gathercole, \& Papagno, 1998). It is critical for 
children learning their first language and for adults learning a new one. The capacity for listening to and repeating language is a good predictor of new vocabulary acquisition (Gathercole \& Baddeley, 1993).

\subsubsection{Visuospatial Sketchpad}

The Visuospatial sketchpad is a system responsible for creating, manipulating, and processing visual and spatial information coming from external input as well as from our mind. Its greater complexity makes the visuospatial sketchpad harder to investigate than the other components of the model. Just like the phonological loop, this component is composed of two subcomponents: visuospatial storage and a visuospatial rehearsal process. Both operate similarly to their analogs in the phonological loop. Visuospatial storage can hold three or four items for a few seconds and is divided in two sorts: visual (also called the visual cache, in charge of retaining shapes and colors) and spatial (also called the inner scribe, responsible for the storage of motion and direction) (Logie, 2011). The spatial subcomponent needs an active rehearsal system in order to hold movements and dynamic information (Olive, 2004). It is easier to remember structured visuospatial information than unstructured, and better recall may be provided by the visuospatial rehearsal system because it encodes visuospatial information into verbal data. The visuospatial rehearsal process is necessary for the retention of visuospatial information, and, as indicated above, it transforms visual or spatial data into verbal code by means of verbalizing the names of the objects or locations. Not all visual information is suitable for verbal encoding. During the verbal transformation, some visual or spatial data can be lost in the process (Baddeley, 2003b). However, research indicates that the visuospatial sketchpad can work along with the phonological loop to process both auditory and visual stimuli without either of the processes affecting the performance of the other (Denis, 
Logie \& Cornoldo, 2012). Any healthy individual is able to drive a car and speak and understand the conversation with the passenger. Baddeley (1996b), considered that the visuospatial sketchpad is more complex and demands more resources from the central executive (described below) than the phonological loop.

It has been proposed that the visuospatial sketchpad may have a role in geospatial orientation, understanding complex mechanical instructions, identification of objects and how to use them (Baddeley, 2003b), control and production of physical movement (Logie, 1996), and during reading, encoding words and letters allowing the reader to not lose his/her place in the text (Baddeley, 1986). Neuropsychological and neuroimaging studies show the distinction between verbal and spatial systems; distinct neural pathways are involved in each. Some brain damage patients have shown severe problems in rotating imaging tasks (spatial imagery), but they have preserved the capacity to perform tasks where nonspatial mental imagery is needed, whereas other patients have shown the reverse pattern (Farah, Hammond, Levine, \& Calvanio, 1988; Hanley, Young, \& Pearson, 1991).

\subsubsection{Central Executive}

Baddeley (2003b) said that the central executive is the least understood component of the model despite being the most important. This essential component is the system that makes the working memory "work", and it is the heart of the model and responsible for managing the other three components. It is necessary for regulating and manipulating cognitive processes involved in working memory; the central executive is crucial when individuals perform two tasks at the same time. In addition, this system works as a link between the other components by selecting information from diverse sources, but it does 
not have storage capacity by itself. Throughout his career, Baddeley (1996a, 2012) described it as an "attention controller" and reported a number of functions of the central executive:

- Selects relevant stimuli, rejecting unimportant information and determining when and which storage buffers are deposited.

- Coordinates performance on simultaneous cognitive tasks, integrating and allocating the information between different parts of the working memory system.

- Controls more cognitive processes, not only working memory, although most of them are related to it.

- Selects and executes plans and strategies.

- Activates, retrieves, and manipulates information from long-term memory, forming new associations. Moreover, helps to encode new information into long-term memory.

- It is activated when automatic processing breaks down and a more conscious control mechanism is needed.

\subsubsection{Episodic Buffer}

Over the years since the model was postulated, problems in trying to explain the interaction of the working memory with the long-term memory and the process of "chunking" (the ability to transform a number of items into only one for easier storage) showed the need to integrate a new component (Baddeley, 2000). Thus, the episodic buffer was created, a system able to store information in a multi-dimensional code. It is a limited-capacity component that is accessible to conscious awareness and capable of integrating information from perceptual sources and other memory systems and maintain these representations. This new component would explain how verbal and spatial information could be combined. Baddeley, (2000) said that the episodic buffer is controlled by the central executive and stores episodes whereby information is integrated across space and time. Namely, the system links the information across domains to form 
integrated units of visual, spatial, and verbal information with time sequencing. The buffer can facilitate problem-solving by creating new cognitive representations and modelling the external conditions. Moreover, the episodic buffer is important for learning because it integrates visual and verbal information, encoding them in the long-term memory. The actual functions of the episodic buffer were attributed previously to the central executive. Therefore Baddeley (2006) acknowledges this component as a fragment of it.

\subsubsection{The latest version of the model}

In 2011, after a number of new studies, Baddeley, Allen, and Hitch revised the model and included missing sensory modalities, in addition to phonological and visuospatial, shown by empirical research data. In Figure 3, we have reproduced the latest version of the model. The episodic buffer is now the only component controlled by the central executive. The phonological loop and the visuospatial sketchpad are only connected to the episodic buffer in charge of integrating the information, coordinating the "orders" coming from the central executive, and binding data from several dimensions into chunks. They speculate that the episodic buffer provides conscious access to the phonological loop and the visuospatial sketchpad. They also speculated without evidence that smell and taste have access to the buffer. The phonological loop and the visuospatial sketchpad have been relegated to lower level buffers, allowing visual, spatial, kinesthetic, and tactile data to be combined in the visuospatial sketchpad. Meanwhile, in the phonological loop, language-related information such as speech, writing, lip reading, and sign language could be manipulated (Baddeley, Allen, \& Hitch, 2011). Baddeley and colleagues concluded their work by accepting that their reformulation of the model raised further questions that had to be investigated, allowing subsequent research to help with the 
evolution of the model. To explain individual differences in visuospatial task efficiency, they suggested the existence of two attentional components in serial visual working memory. A new system is recruited to help the attentional system when its capacity is filled; the central executive provides the necessary resources to the episodic buffer to work efficiently (Allen, Baddeley, \& Hitch, 2014).

Figure 3: Diagram of the Baddeley, Allen and Hitch (2011) revised multi-component model.

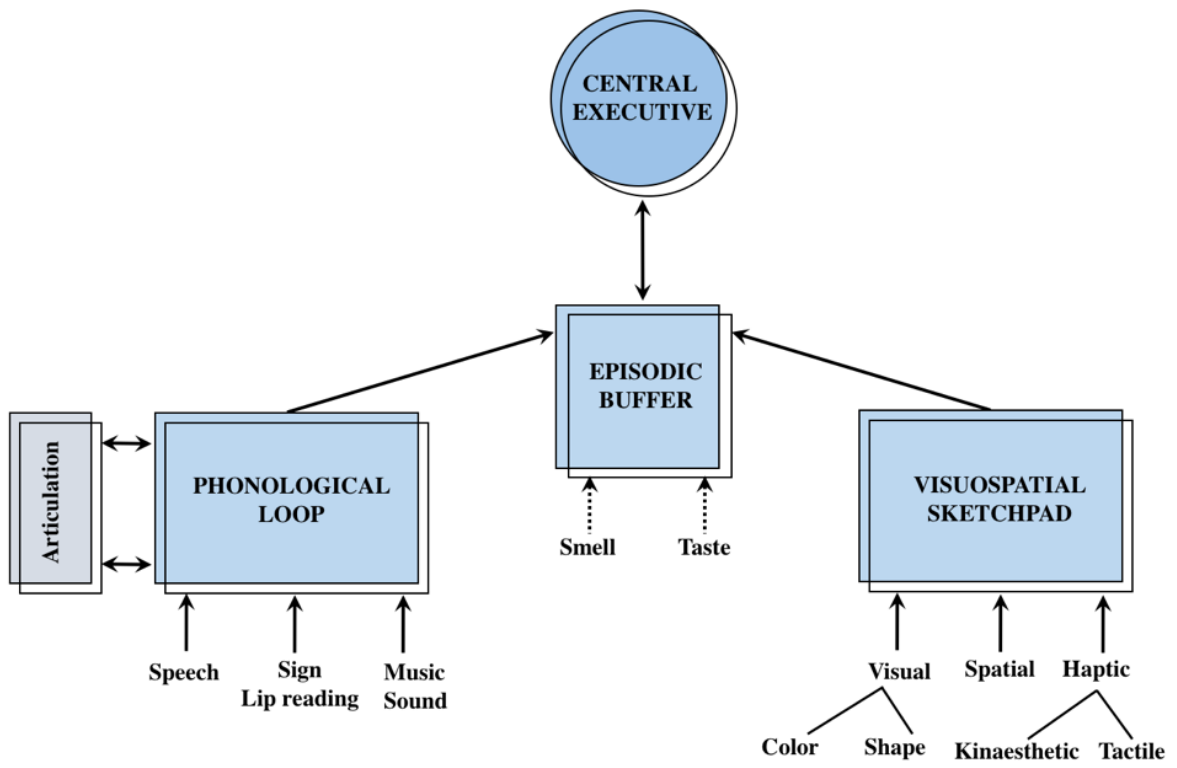

\subsubsection{Neuroanatomical localization of the components}

Baddeley's model is a conceptual model used to explain the results obtained from psychological and neuropsychological research in humans that serves to illustrate the mechanisms of diverse cognitive processes, including language comprehension, thinking, reasoning, and decision making. Thus, it is difficult to assign a certain brain area to each of its component. However, neuroimaging and neuropsychological studies have provided support for the multi-component model of working memory. While performing unlike working memory tasks, different brain areas are activated, as shown by a large number 
of neuroimaging studies. Phonological, visuospatial, and executive functions of working memory switch on distinct brain regions (Hedden \& Yoon, 2006). In Figure 4, we have tried to map the areas associated with each component, based on existing empirical information developed in the next paragraph.

Figure 4: An approximation to the cerebral locations of the different components of the multi-component model guided by neuroimaging and neuropsychological research. Based on Baddeley (2003; Figure 6). Subcortical areas are not represented. Green color: central executive (CE), red color: episodic buffer (EB), yellow color: phonological loop (PL) and blue color: visuospatial sketchpad (VS).
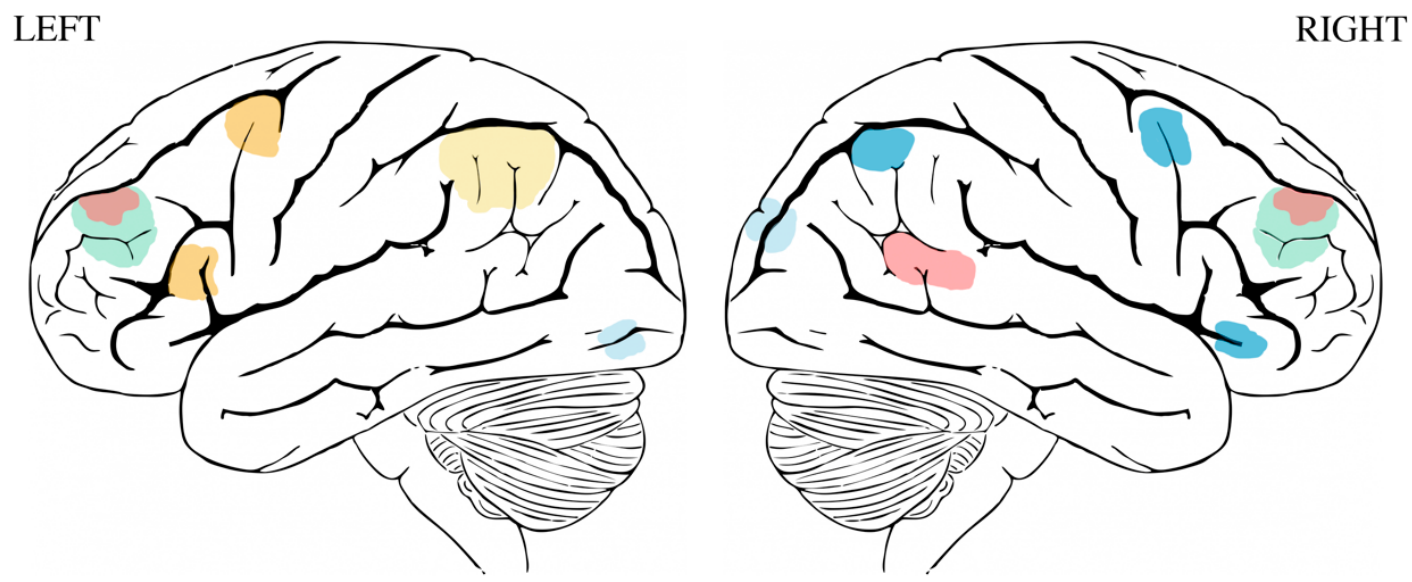

CE

EB: Cognitive functions

EB: Long-term related functions
PL: Phonological store

PL: Articulatory rehearshal
VS: Visuospatial store

VS: Visuospatial rehearshal

Regarding the phonological loop, each subcomponent is located in different brain regions, commonly in the left hemisphere. The phonological store relies on the left temporoparietal region (Brodmann area (BA) 40), and articulatory rehearsal depends on Broca's area (BA 6/44) in the frontal lobe (Baddeley, 2003a; Baldo \& Dronkers, 2006). Studies of patients with brain damage supported these statements (Vallar \& Papagno, 2002). In addition, research has identified visuospatial working memory as being mainly, but not exclusively, dependent on the right hemisphere, especially in frontal, parietal, and 
occipital areas. Specifically, visuospatial rehearsal may be situated in the right inferior parietal cortex (BA 40), right premotor cortex (BA 6), and right inferior frontal cortex (BA 47). However, the anterior extrastriate occipital cortex (BA 19) has bilaterally been related to visuospatial storage (Awh \& Jonides, 2001; Postle, Brush, \& Nick, 2004). There is also neurological evidence for episodic buffer. Originally, Baddeley (2000) identified its anatomical location in frontal areas due to its role in executive processing and its connections with the central executive. A more recent neuroimaging study supported the notion of an episodic buffer processing that is strongly related to long-term memory and executive functions (Rudner, Fransson, Ingvar, Nyberg \& Rönnberg, 2007). The authors found activation in the bilateral dorsolateral prefrontal cortex (DLPFC; BA 9/46), in the right middle temporal lobe (BA 21), and in the left hippocampus (BA 28). In her work, Balconi (2013) suggested that DLPFC allows to utilize old stored data (episodic memories) to manipulate new information (working memory) by activating the episodic buffer. Therefore, episodic buffer executive processes would activate prefrontal areas, whereas long-term memory related functions would turn on temporal regions.

Neuroimaging and lesion studies have connected executive functioning with the frontal lobes (Kane \& Engle, 2002; Smith \& Jonides, 1997; Wager \& Smith, 2003). More recent research has focused on the bilateral DLPFC (BA 9/46) for the control of cognitive processing, stimuli selection, attention focus, and adequate responses (Lara \& Wallis, 2015). Evidence with patients supports the notion of a central executive (Baddeley, Baddeley, Bucks, \& Wilcock, 2001; Müller \& Knight, 2006). In their study, Baddeley and colleagues (2001) demonstrated that patients with Alzheimer's disease presented cognitive impairment due to a dysfunction of the central executive. Alzheimer's disease patients were clearly impaired when performing a dual-task (an auditory digit span and a 
visuomotor tracking task), compared to healthy elderly participants. The central executive has also been investigated by evaluating the behavior of frontal lobe brain damage patients. These patients present a distinctive behavior called perseveration where they are unable to change their conduct when they have already started. This shows an impairment in the central executive's ability to control attention (Goldstein, 2008).

In conclusion, the Baddeley-Hitch model took a step forward in the working memory field by proposing a multi-component model, and it became the most widely accepted conceptual approach to describe the working memory principle. Lastly, it is necessary to add that the multi-component model has given rise to a large amount of research and is still highly influential. The model has been stimulating investigation on working memory for more than 40 years since it was first proposed. However, from a neuroscience perspective, this model has been criticized because of its specialized dedicated storage proposal and its unitary view of the working memory system. There are many types of information that need to be manipulated in the working memory to guide behavior, and there could not be enough independent buffers to provide space for all of them. Later cognitive models postulated that information is processed through the action of cerebral structures that reproduce the data being held, instead of passing the information to a specialized buffer. The concept of working memory as a unitary system that is in charge of storage and processing is beginning to run out of steam in the cognitive neuroscience field (D’Esposito, 2007).

\subsection{Cowan's Embedded Processes Theory}

Nelson Cowan is an American psychologist who also studied the concept of working memory. He proposed a different working memory model (Figure 5) in 1988, trying to 
explain the weakness that, in his view, the Baddeley's model had. He defined working memory as "cognitive processes that are maintained in an unusually accessible state" (Cowan 1999, p. 62). This definition of working memory means that Cowan's model focus on underlying cognitive processes that take place during task performance, unlike Baddeley's model, which describes the modularity and components of working memory. In his opinion, Baddeley's model could not explain what happens with other information (touch, smell, taste and abstract ideas) apart from verbal and spatial. In Cowan's model, the phonological and visuospatial stores are instances of the temporary activation of longterm memory information, and they are able to hold sensory data of all modalities (Cowan, 2010). He tries to explain a single way of functioning, regardless of the type of stimulus or input. This author closely related working memory with long-term memory; in his model, the two processes have a close interaction and mutual interdependence.

According to the embedded processes model of working memory, the memory organizes at two levels: the long-term memory and the focus of attention. The long-term memory would be the first level, with unlimited capacity, whereas the focus of attention is limited to $4 \pm 1$ chunks or episodes (each can contain more than one item), depending on the difficulty of the task. Elements can be activated voluntarily or involuntarily (Cowan, 2009). Moreover, this theory distinguishes two more main elements: the activated memory (a temporal activation sublevel of long-term memory) and the central executive (controls the attention). As with the Atkinson-Shriffrin Model, when a stimulus arrives to the system, it is held for milliseconds in the sensory store. Then, the stimulus is transferred to the activated memory or to the focus of attention. The information can be maintained in the focus of attention by means of strategies like rehearsal. The long-term memory interacts with the working memory in order to form new episodic relations 
between new information and that which already exist, encoding it sensorially or abstractly. The central executive is able to look into the long-term memory and introduce new information into the focus of attention. It can also replace information present in the focus with other information. In this model, the different processes of activated memory, central executive, and focus of attention work together to hold information in the mind for a short period of time to perform diverse cognitive tasks (Cowan, 2010).

Figure 5: Original Cowan's model of the information-processing system. Extracted from Cowan (1988).

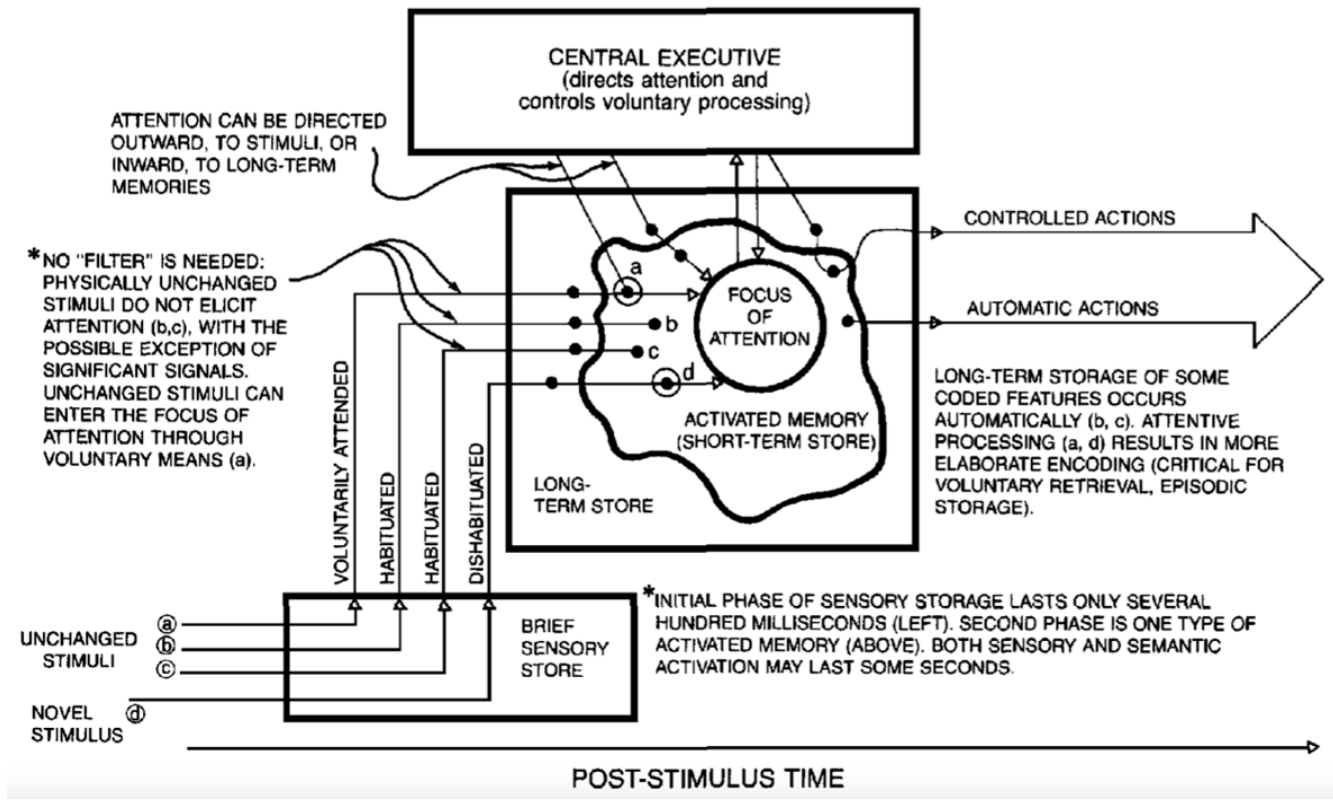

Baddeley wrote about Cowan's view that both models "agree on most issues but differ in our terminology and areas of current focus. I see Cowan's model as principally concerned, in my terminology, with the link between the central executive and the episodic buffer." (Baddeley 2012, p. 20). He continues by explaining that Cowan does not give an appropriate justification about what he postulates, with a more exhaustive analysis of the processes being necessary. Even so, both theories agreed on some aspects, such as the existence of one passive storage component and one active processing component in working memory. 


\subsection{A facet model of working memory}

Another theory emerged that focuses on the executive aspects of working memory. Oberauer and colleagues $(2000,2003)$ postulated a facet theoretical framework with two working memory dimensions (or facets). On the one hand, there is a content facet that differentiates two main general factors: verbal and numerical working memory and visuospatial working memory. This hypothesis is consistent with the differentiation of two domain-specific slave systems in Baddeley's model. On the other hand, a functional facet is formed by three different processes that together include most of the working memory functions: simultaneous storage and processing, supervision (executive functions), and coordination of information elements into structures. Simultaneous storage and processing is the main function of working memory; it keeps the information for a short period of time and processes it quickly and efficiently. In addition, supervision involves monitoring the processes and actions initiated, eliminating unimportant and distracting information, and performing selective activation of important information. In this model, coordination is the capacity to construct new links between items and integrate relations into structures (Oberauer, Süß, Schulze, Wilhelm \& Wittmann, 2000; Oberauer, Süß, Wilhelm \& Wittman, 2003). Evidence is provided for the facet model in studies on individual differences by demonstrating the three functional processes into which working memory can be subdivided (Buehner, Mangels, Krumm \& Ziegler, 2005; Oberauer et al., 2003).

According to Cowan (2010), Oberauer accepted the basics aspects of the Embedded Processes Theory, but he made some changes. He believed that the focus of attention is only able to hold one item at a time. In addition, he divided activated long-term memory into the three functional processes described previously, instead of two. However, 
although there is some disagreement about the terminology, both theories postulate the same thing. When someone has to remember some information, the long-term memory representations of these items are accessed during the process of perceptual recognition, and then they are held in an elevated state of activation (with attention) until these data are no longer needed to perform any task (D’Esposito \& Postle, 2015).

\subsection{Component processes view of working memory}

In recent years, a new theoretical framework has emerged to explain the working memory processes taking into account the new neuroimaging and neuropsychological evidence. This new paradigm analyzes working memory as a component processes instead of one whole working memory system. Thus, working memory is not considered a single construct according to this view, but it is considered a computational and cognitive function resulting from several combinations of processes that can be found in other cognitive functions (Eriksson, Vogel, Lansner, Bergström \& Nyberg, 2015; Fuster, 2009; Moscovitch and Winocur, 2002; Nyberg \& Eriksson, 2016).

Figure 6: Representation of the different working memory processes and interactions in a process-based view. Extracted and adapted from Nyberg and Eriksson (2016, p. 4, Figure 1). "Color-coding: perception in light blue, action in orange, motivation/drive in red, long-term memory representation and consolidation in blue, and, in yellow, the working memory processes that will be discussed. Arrow 2 represents reverberating activity in frontal, parietal, and representational areas during maintenance. Arrow 3 represents consolidation of working memory information into long-term memory via interactions with the medial-temporal lobe system. Arrow 4 represents associations between manipulation networks, mainly in the dorsofrontal cortex, and frontoparietal maintenance/ attention processes. Arrow 5 represents nigrostriatal dopaminergic neurotransmission and striatocortical interactions during working-memory updating. Arrow 6 represents diffuse dopamine gating signals from the ventral tegmental area (VTA) to the frontal cortex. Arrow 7 represents emotional input to rostrofrontal cortical regions, and arrow 8 represents how neurons in the rostrofrontal cortex, coding for cognitive and motivational context, influence other working-memory networks to 
support goal-directed behavior. Transparency of ellipses indicates major subcortical nodes for updating (striatum), consolidation (hippocampus), and motivation (amygdala, brain stem, and hypothalamus). SN, Substantia nigra."

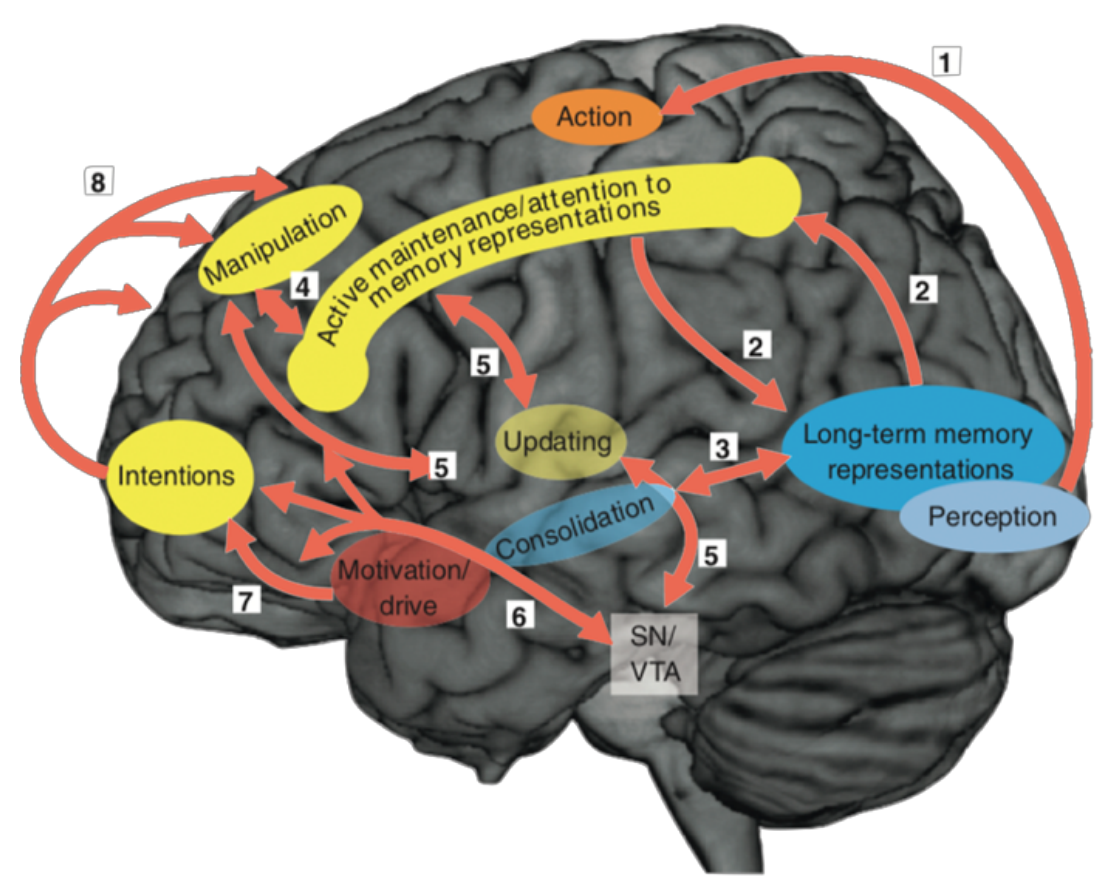

This component processing view of working memory bases its affirmations on neuroimaging and neuropsychological research and lesion studies. Nyberg and Eriksson, (2016) now defined working memory as "temporary online maintenance of information for the performance of a task in the (near) future, but more broadly to also include manipulation and updating of the aforementioned information, as well as coordinating behavior when multiple goals are active." (Nyberg \& Eriksson, 2016, p. 3). According to this proposal, the working memory operation can be explained by three of these processes: maintenance, manipulation, and updating (Figure 6; Eriksson et al., 2015; Nyberg \& Eriksson, 2016). In accordance with this theoretical framework, several different brain regions are involved in working memory because it is formed of different processes. Therefore, as Figure $\mathbf{6}$ shows, maintenance involves prefrontal and parietal areas, whereas manipulation only activates the prefrontal cortex, especially the 
dorsolateral part. Updating, in turn, is illustrated by arrow 5 in Figure 6, connecting the basal ganglia with frontoparietal cortices. In this view, the long-term memory is closely related to working memory due to the activity of the hippocampus because this area makes it possible to consolidate the information utilized in working memory (Nyberg \& Eriksson, 2016). More detailed information about the brain areas involved in working memory will be presented in the next section.

\subsection{Conclusion}

Since the concept of working memory was officially established, many years of research and discussion have passed. The concept has evolved from both neuroscience and psychology, and theories and models of working memory have steadily emerged to give, at each point in time, a better account of working memory processes. Thanks to empirical evidence collected through the different behavioral, neurophysiological and neuroimaging studies, a more comprehensive picture of working memory is being formed. However, none of the working memory models or theories seems to be complete; they do not explain all the aspects of this complicated construct. The large number of various models and theories demonstrate the different views of the nature, structure, function, and uses of working memory. In the past few years, advances in neuroimaging techniques and data analysis have provided more understanding about the working memory process, viewing it as a non-unitary system that allows processing and storage. Nevertheless, there is no agreement in the scientific field: specific mechanisms involved in working memory are still fiercely debated. Further investigations are still needed to provide evidence for the continuous updating of our models of the way working memory works. 


\section{Working memory and the brain}

Throughout the years, the neural correlates of working memory have been investigated in both animals and humans by means of diverse research techniques. Initially, researchers used patients who were brain injured in different cortical areas, analyzing their behavior in order to compare it with that of healthy people and other types of patients and trying to find the neural mechanisms of working memory. Most recently, with the advances in neuroimaging techniques, the field has come to expand and understand the complexity of the neural networks and connections of working memory. Functional magnetic resonance imaging (fMRI), electroencephalography (EEG), transcranial magnetic stimulation (TMS), positron-emission tomography (PET), and genetic analysis are some of the non-invasive technologies that have been used to investigate the neural basis of working memory in humans. The following sections are dedicated to briefly reviewing the evidence related to the role of the different brain areas and networks engaged during working memory, as they have been studied in neuroimaging studies (preferentially fMRI studies in humans).

\subsection{Brain areas related to working memory}

Localization of brain functions in humans has become much easier with the arrival of brain imaging methods. Normally, in task-related studies, the participants' brains are scanned while performing a working memory task with a control condition. Then, the conditions are compared to identify which brain areas are selectively activated or deactivated during performance of the working memory condition. Depending on the task and the type of stimuli used in it, some areas will be activated or not. But there are common regions that are always activated: the areas in charge of working memory 
processes such as manipulating, monitoring, updating, or selective attention. Neuroimaging data can provide a map of brain regions that mediate task performance. However, task-related functional imaging reveals activity in different brain regions involved in the task, but it is difficult to conclude that that specific region is necessary for a cognitive process. Lesion studies can be useful in this situation because they provide a concise way to define which areas are physically fundamental for a cognitive process.

Working memory processes are the result of the interaction between several brain areas. Various task-related and lesion neuroimaging studies have found that all the working memory tasks generally activate different areas that take part in the frontoparietal network (Figure 7) (Constantinidis \& Klingberg, 2016). In a meta-analysis by Nee et al. (2013), the brain regions involved in executive processes of working memory were studied, and a number of frontal and parietal areas were described. Figure 8 presents their outcomes. It is a useful and graphic image that summarizes the results of a large number of working memory investigations and can provide an indication of the frontoparietal areas involved in executive processes of working memory. Nevertheless, not only frontoparietal areas are involved in working memory because subcortical and temporal regions have also been related to different working memory processes, as explained below.

Figure 7: The main effect across 189 working memory studies on bilateral frontoparietal activation. Extracted from a meta-analysis focused on the study of the neural correlates of working memory, carried out by Rottschy et al. (2012, p. 835, Figure 1).

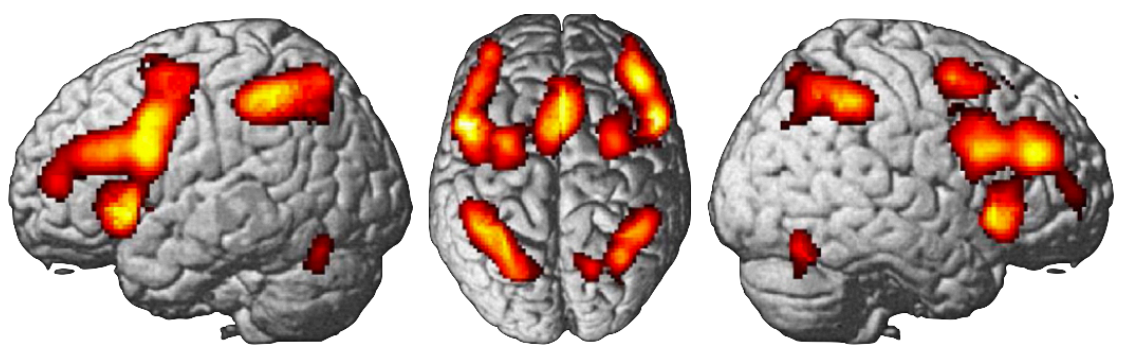


Figure 8: Representation of regions involved in executive processes of working memory. Extracted from a meta-analysis focused on the study of the brain regions involved in executive processes of working memory by Nee et al. (2013, p. 267, Figure 1). "Colors denote gyral definitions derived from the AAL atlas (Tzourio-Mazoyer et al., 2002). Prominent sulcal and gyral regions are denoted with bubbles. Literature associated with each region is listed. More general anatomical labels are associated with one or more gyri: midventrolateral prefrontal cortex $(\mathrm{VLPFC})=$ inferior frontal gyrus (IFG), pars triangularis and IFG, pars opercularis; dorsolateral prefrontal cortex (DLPFC) $=$ middle frontal gyrus (MFG); premotor cortex = precentral gyrus (preCG); posterior parietal cortex $(\mathrm{PPC})=$ superior parietal lobule (SPL) and inferior parietal lobule (IPL); temporal parietal junction $(\mathrm{TPJ})=$ supramarginal gyrus $(\mathrm{SMG})$. Other abbreviations: inferior frontal sulcus (IFS); inferior frontal junction (IFJ); superior frontal sulcus (SFS); intraparietal sulcus (IPS)."

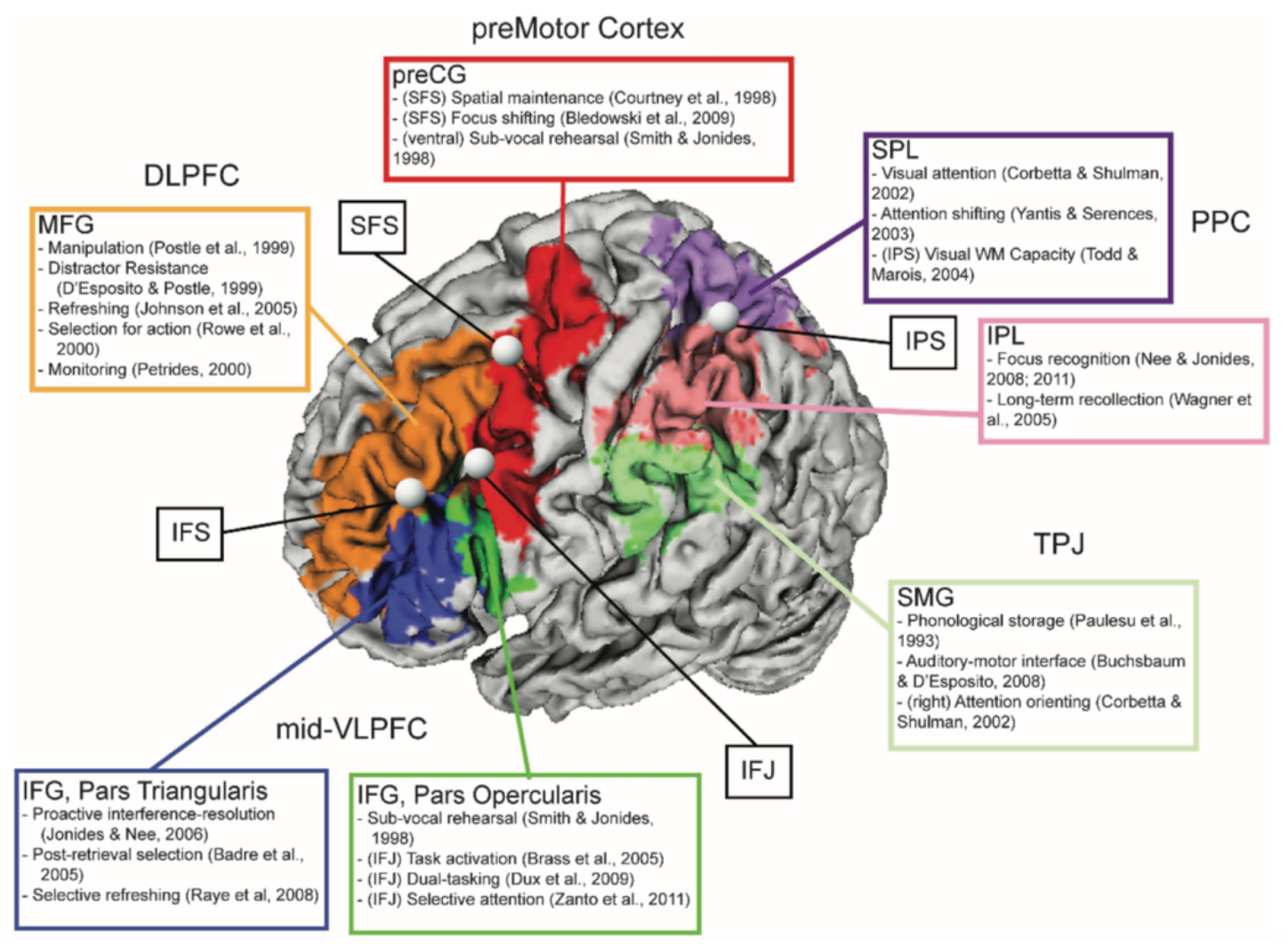

\subsubsection{The role of the frontal lobe}

The frontal lobe is located at the front of each cerebral hemisphere, and it is the largest of the four major lobes of the cerebral cortex in the human. It takes up about a third of all the cerebral cortex. Years of research have provided plenty of clinical evidence about the importance of the frontal lobe in working memory. Different areas of the human frontal 
cortex have been related to working memory performance. These areas include the superior frontal gyrus (SFG), the supplementary motor area (SMA), inferior frontal gyrus (IFG), and the DLPFC. Moreover, although they are not strictly frontal areas, the anterior insula (AI) and the anterior cingulate cortex (ACC) are also involved (Nee et al., 2013; Rottschy et al., 2012). These areas have been related to different functions underlying the performance of working memory tasks.

The main frontal region related to working memory in the frontal lobe is the prefrontal cortex. In the human brain, the prefrontal cortex covers the front part of the frontal lobe and occupies 29\% of the cerebral cortex (Fuster, 2008). Some of the earliest working memory neuroimaging studies demonstrated the importance of this region for this construct (D’Esposito et al., 1995; Goldman-Rakic, 1987; Jonides et al., 1993). The prefrontal cortex is so important in working memory that has been appointed as the neural substrate of the working memory (Müller \& Knight, 2006). Previously, the prefrontal cortex was considered a storehouse for the information about the relevant stimulus necessary for a task or operation. Neuroimaging studies in humans and animals contradicted this view (see D'Esposito \& Postle, 2015; Funahashi, 2017 for a review). Currently, it is known that the prefrontal cortex receives diverse information through bottom-up signaling through cortical and subcortical connections, and through top-down signaling, the prefrontal cortex commands the processes of other cortical and subcortical regions (Edin et al., 2009; Funahashi \& Andreau, 2013; Hwang, Velanova, \& Luna, 2010; Johnstone, van Reekum, Urry, Kalin, \& Davidson, 2007; Lee \& D’Esposito, 2012; Tomita, Ohbayashi, Nakahara, Hasegawa, \& Miyashita, 1999). There are two types of top-down control signals that depart from the prefrontal cortex, according to D'Esposito \& Postle (2015): signals that modulate gain by either enhancing task-relevant information 
or suppressing task-irrelevant information, and signals that can modulate the selectivity of information represented in posterior cortical regions. Updating and monitoring of information, stimuli selection and inhibition, attention focus and switching, coordinating and integrating information and providing adequate responses are examples of executive functions linked to prefrontal cortex activity (Alvarez \& Emory, 2006; Lara \& Wallis, 2015; Postle, 2006; Stuss \& Alexander, 2000). These executive functions fit the functions of the central executive proposed by Baddeley very closely.

The prefrontal cortex is a region that comprises severe different architectonic areas. One of the areas that forms part of it is the DLPFC. The DLPFC is a functional structure situated in the middle frontal gyrus in the human brain and corresponds to Brodmann's areas 9 and 46. There has been a debate in cognitive neuroscience about the functional organization of the DLPFC and its role in working memory: two different points of view have been expressed. On the one hand, domain-general models postulate that DLPFC is specialized in some type of working memory function, specifically monitoring and manipulating cognitive information. On the other hand, domain-specific models maintain that the DLPFC is especially prepared to process visuospatial information, making it possible to mentally represent coordinates within the spatial domain (Barbey, Koenigs \& Grafman, 2013). Although this region is not exclusively in charge of executive functions, the new neuroimaging and clinical research with animals (the vast majority with monkeys) and humans supports the domain-general models. The DLPFC plays a crucial role in reasoning, monitoring, and manipulating cognitive items in working memory (Balconi, 2013; Barbey et al., 2013; Eriksson et al., 2015; Petrides, 2005; Petrides, 2000). The fact that the DLPFC has a more unified role in cognition could lie in its connectivity with other regions (Curtis \& D'Esposito, 2003). According to Curtis and D'Esposito 
(2003), the DLPFC plays a role in the maintenance of data by directing attention to internal representations of sensory stimuli and motor plans that are stored in more posterior regions. Posterior parts of this area may take part in more specific roles, whereas anterior parts control basic executive processes (Constantinidis \& Klingberg, 2016; D'Esposito \& Postle, 2015). Additionally, it has been suggested that DLPFC working memory functions are lateralized. The left part of the DLPFC would be in charge of verbal working memory, whereas the right part would be specialized in spatial working memory (Barbey et al., 2013; Nee et al., 2013; Owen, McMillan, Laird, \& Bullmore, 2005; ReuterLorenz et al., 2000; Smith, Jonides, \& Koeppe, 1996; Wager \& Smith, 2003).

Regarding the IFG, it is known that it also forms part of the prefrontal cortex (BA 44, 45 and 47), and neurocognitive research gives this region in the brain a role in working memory; specifically, it has been related to inhibition. Tops and Boksem (2011) reviewed evidence that the IFG is involved in elaborate attentional and working memory processing. In addition, the authors postulated that the processing may take different forms and different effects depending on the task performed: it may facilitate fast and accurate responses, when long complex processing is needed to increase exactness it may cause slow responding, and, lastly, when long complex processing interferes with the processing of the next stimulus, it may mediate with the accuracy and speed of next-trial performance (Tops \& Boksem, 2011). The left IFG has a more consistent activation on tasks that use verbal stimuli (Rottschy et al., 2012), whereas the right IFG has been strongly related to the inhibition of responses by sending top-down signals to the basal ganglia and subthalamic nucleus in order to detain inappropriate responses (Aron, 2011; Aron, Robbins \& Poldrack, 2004). With regard to SMA (BA 6), this area has been related to the planning of sequences of movement, word generation, motor learning, and motor 
activation of the hand. Likewise, the superior part of the frontal cortex plays a role in working memory. In a meta-analysis by Nee et al. (2013), which studied the brain regions involved in executive processes of working memory, the authors concluded that the SFG has a strong association with spatial content. Therefore, this area of the frontal lobe seems to be involved in spatial attention in working memory.

The AI and the IFG have frequently shared cognitive functions (Nee et al., 2013; Tops \& Boksem, 2011). The AI also has a role in inhibition (Wager et al., 2005), and it is involved in temporary detection of relevant stimuli, salience, and attention (Cauda et al., 2011), task-set maintenance (Dosenbach et al., 2007), and starting attentional control signals working together with the ACC (Menon \& Uddin, 2010). Neuroimaging research has shown that the ACC has various relevant functions in working memory. Apart from collaborating with the AI, its functions include motivation, modulation of attention, monitoring competition, complex motor control, error detection, and anticipation of cognitively demanding tasks (Bush, Luu \& Posner, 2000).

\subsubsection{The role of the parietal lobe}

Just behind the frontal lobe and the central sulcus and above the occipital lobe, the parietal lobe is located. A large proportion of the working memory based search in cognitive neuroscience has focused on frontal regions, especially when it comes to executive functions of working memory. However, it has been recognized that the parietal regions are also important in executive processes (Collette, Hogge, Salmon \& Van der Linden, 2006). Usually, experts subdivide the parietal cortex into the lateral and medial cortex. In turn, the medial parietal cortex corresponds to the post central gyrus area, whereas the lateral cortex is subdivided into dorsal and ventral areas. The intraparietal sulcus and the 
superior parietal cortex (SPC) are included in the dorsal part of the parietal lobe, whereas the inferior parietal cortex (IPC), formed by the supramarginal gyrus and the angular gyrus, compose the ventral part of the lateral cortex (Caspers et al., 2011; Humphreys \& Lambon Ralph, 2015; Mars et al., 2011; Nelson et al., 2010; Uddin et al., 2010). The SPC and IPC are the brain areas that have been related to different functions underlying the performance of working memory tasks (Figure 8). Formerly, a specific spatial role was given to the parietal areas, but we now know that these areas contribute to working memory with different functions, above all involved in attentional processes of working memory.

On the one hand, posterior to the postcentral sulcus and above the intraparietal sulcus is the SPC (BA 5 and 7). Attention and visuospatial perception are functions that have been related to this area, including the illustration and manipulation of data (Johns, 2014). Although the SPC demonstrates a preference for spatial content, it has been demonstrated that it is also activated across different functions, not only with spatial content, but also with verbal and phonological stimuli (Collette et al., 2006; Nee et al., 2013). Therefore, the SPC has been related to top-down control of attention, numerical calculation (Humphreys \& Lambon Ralph, 2015), object location and verification (Rottschy et al., 2012), distractor resistance, updating (Nee et al., 2013), ordering and manipulation of acquired facts (Koenigs, Barbey, Postle, \& Grafman, 2009) in the literature on neuroimaging studies with healthy and lesioned subjects. The posterior region of the SPC (BA 7) plays a role in the essential control of the focus of attention (Collette et al., 2006).

On the other hand, the IPC (BA 40), which lies below the horizontal portion of the intraparietal sulcus and behind the lower part of the postcentral sulcus, has a role in the 
attentional processes of working memory when an attentionally-demanding upholding strategy is used (Berryhill, Chein, \& Olson, 2011). Likewise, the IPC has been strongly related to the shifting necessary to maintain activated, for example, auditory and visual information simultaneously (Nee et al., 2013; Collette et al., 2006). Moreover, in their review, Collette et al., (2006) found more activity in parietal areas than in frontal areas during switching demands. As with the DLPFC, literature suggests that working memory functions are also lateralized in the parietal regions; spatial content commonly activates the right hemisphere more, whereas verbal working memory performance preferentially activates the left part (Eriksson et al., 2015; Nee et al., 2013; Owen et al., 2005).

In sum, it seems clear that working memory is not localized to a single cerebral region. The prefrontal cortex has a crucial role due to its interactions with other brain regions, as demonstrated by neural studies. The diverse parts of the prefrontal cortex may have different roles, always related to executive function, such as task rules, goals, or abstract representations of categories (D'Esposito \& Postle, 2015). The DLPFC has been more strongly related to reasoning, monitoring, and manipulating, whereas the IFG has been involved in inhibition and verbal processing. In addition, the AI has a role in salience and attention, and the ACC has functions of motivation, error detection, and anticipation of cognitively demanding tasks. The prefrontal cortex also has reciprocal connections with the parietal cortex during working memory processes. Specifically, the SPC and the IPC participate mainly in attentional functions. Even so, the working memory process does not operate without the intervention of other cerebral areas described in the following section. 


\subsubsection{The role of subcortical areas}

There are some subcortical areas that play a role in working memory. It is not their function itself, but their connection with the cortex (mainly with the frontal cortex), that takes part in the working memory process. The cerebellum, the thalamus, and the basal ganglia are the subcortical structures commonly activated during performance of working memory tasks (Constantinidis \& Klingberg, 2016; Nee et al., 2013; Owen et al., 2005; Rottschy et al., 2012).

Situated in the posterior cranial fossa, the cerebellum is a structure with a firm folded layer of cortex and white matter below it. It has been known for a long time that the cerebellum has an important role in control movement (Glickstein, 1993; Holmes, 1939). However, in recent years, evidence from neuropsychological (Schmahmann, Weilburg \& Sherman, 2007) and neuroimaging (Stoodley, 2012) studies have reported that the cerebellum is involved in different non-motor behaviors (see O'Halloran, Kinsella \& Storey, 2012 for a review). Therefore, the literature suggests that the cerebellum has comparable contributions to both cognitive and motor processes (Schmahmann, 2004). Research with clinical populations with cerebellar lesions have shown that these patients had deteriorated accuracy on working memory tasks (Peterburs, Bellebaum, Koch, Schwarz \& Daum, 2010; Ravizza et al., 2006). In addition, neuroimaging studies have shown cerebellar activations during performance of working memory tasks in healthy subjects (Owen et al., 2005; Stoodley \& Schmahmann, 2009). In the cerebellum, it has been suggested that language specialization is situated in the right hemisphere, whereas spatial content is left-hemisphere dominant, exactly the opposite of what occurs in the cerebral hemispheres (Andreas et al., 2004; Marien, Engelborghs, Fabbro \& De Deyn, 2001). Tomlinson, Davis, Morgan \& Bracewell (2014) demonstrated in their experiment 
that the application of continuous theta burst stimulation in the right cerebellar hemisphere caused an impairment in verbal working memory. Moreover, different studies have found that the superior part of the cerebellum participates during encoding, and the inferior part is active during maintenance (Chen \& Desmond, 2005; Keren-Happuch, Shen-Hsing, Ringo Ho, \& Desmond, 2012). All these working memory processes that the cerebellum has may be possible due to its connections with the prefrontal cortex (Hayter, Langdon \& Ramnani, 2007; Vandenvert, 2009).

Several meta-analyses and reviews have reported activations in the thalamus while conducting working memory tasks (Owen et al, 2005; Nee et al., 2013; Rottschy et al., 2012; Constantinidis \& Klingberg, 2016; Nyberg \& Eriksson, 2016). The thalamus, located in the forebrain, dorsal to the midbrain, is an ovoid structure that forms the lateral divider of the third ventricle. This structure is a heterogeneous arrangement of wellorganized nuclei. Sensory and motor input signals are received by the thalamus, and it has two-sided connections with the cerebral cortex. Motor, arousal, and mood are some of the functions that the thalamus is involved in, and it also sends neural impulses from sensory data to the cortex (Mai \& Forutan, 2012). In general terms, the thalamus is divided into three parts (anterior, medial, and lateral), and these, in turn, are divided into several nuclei with a specific name depending on their position. All the nuclei have reciprocal projections to the cerebral cortex; there are specific projections between individual thalamic nuclei and cortical areas with certain well-defined sensitive or motor functions, whereas others have less specific information or connect with more widespread areas (Crossman \& Neary, 2007). Therefore, which of these nuclei are related with working memory? In their review, Watanabe and Funahashi (2012) present findings and evidence to support the idea that the mediodorsal nucleus (MD) is involved in working 
memory processes due to its connection with the DLPFC. The MD acts as a support in DLPFC working memory functions. As occurs with the cerebellum, research with clinical populations with MD lesions and neuroimaging studies in healthy subjects have shown impairments in working memory performance in the former group and thalamic activations during performance of working memory tasks in the latter (Watanabe \& Funahashi, 2012).

Finally, interactions have been described between the basal ganglia and the prefrontal cortex throughout the execution of working memory tasks (Frank, Loughry \& O'Reilly, 2001; O'Reilly \& Frank, 2006; Wager \& Smith, 2003). Situated at the base of the forebrain, around the thalamus, the basal ganglia are a combination of nuclei and masses of gray matter, formed by: the striatum (formed, in turn, by the caudate nucleus and the putamen), the globus pallidus, the subthalamic nucleus, and the substantia nigra. This gray matter tissue has strong interconnections with the cortex, the thalamus, and the brainstem. The main function of the basal ganglia is the control of movement and posture, but evidence from research reveals that they are involved in other functions outside the motor sphere. Therefore, diverse cognitive processes have been related to the basal ganglia, including attention, planning, and procedural memory (see Stocco, Lebiere, \& Anderson, 2010 for a review). Regarding working memory, McNab and Klingberg (2008) reported basal ganglia activation, specifically in the putamen and the globus pallidus, at the same time that healthy participants performed a working memory task. The authors related the basal ganglia activity to a relevant information filter in working memory. Moreover, they stated that "frontal and basal ganglia activity exerts attentional control over access to working memory storage in the parietal cortex in humans, and makes an important contribution to inter-individual differences in working memory capacity" 
(McNab \& Klingberg, 2007, p. 103), and they reported frontal and basal ganglia activity as a predictor of working memory capacity. In another study by Moore and colleagues, caudate activity was found during the encoding phase of their working memory task, and the findings are consistent with the aforementioned study giving the basal ganglia a role as a filter, suppressing distractors (Moore, Li, Tyner, Hu, \& Crosson, 2013). In addition, the subthalamic nucleus has been related to the right IFG sending top-down signals in order, once more, to stop inappropriate responses (Aron, 2011; Aron et al., 2004). In addition, activation in the substantia nigra has been found during updating on a working memory task (D'Ardenne et al., 2012), as well as striatal activity (Dahlin, Neely, Larsson, Backman, \& Nyberg, 2008; Lewis, Dove, Robbins, Barker, \& Owen, 2004). All this activity in the basal ganglia during working memory processes indicates that the neurotransmitter dopamine has an important role in this cognitive function. There is evidence that D1 and D2, the two major dopamine receptors, are related to working memory (Cohen, Braver, \& Brown, 2002; Grace, 2000). D1 is related to maintenance, whereas D2 is involved in the updating of working memory (Nyberg \& Eriksson, 2016).

\subsubsection{The role of the temporal lobe}

The temporal lobe is the second largest lobe in the human brain. It is located in the forebrain, just behind the temples. For years, the temporal lobe was not considered important for working memory due to its corroborated relationship with long-term memory (Eriksson et al., 2015). However, in the last decade, some neuroimaging evidence has found temporal lobe activation throughout a working memory task (Axmacher et al., 2007; Constantinidis \& Klingberg, 2016; Eriksson et al., 2015; Nee et al., 2013; Rottschy et al., 2012), suggesting that the temporal lobe may be involved in 
different cognitive domains. Even so, there is no agreement about its exact role in working memory.

In their review, Nyberg and Eriksson (2016) wrote that activations in the hippocampus and medial temporal lobe (MTL) may be associated with the consolidation system of the information maintained in working memory to long-term memory. They based their statements on studies with patients with MTL lesions. Libby et al. (2012) studied whether MTL regions are involved in the pertinent spatial and object information during a working memory task. Their results showed that the perirhinal cortex (PRC), the amygdala, and the temporopolar cortex are involved in transmitting information about the objects encoded in the working memory task, and the parahippocampal cortex (PHC) is activated when information about spatial configuration is present. The authors suggest that processing object, spatial, and object-location merging information during working memory encoding and delay involves the PRC, PHC and the hippocampus (Libby, Hannula, \& Ranganath, 2014). Al this new literature demonstrates that temporal lobe areas are involved in working memory, but, in the absence of a consensus, further investigation is required in this field to determine the exact relationship between them.

\subsection{Conclusion}

Working memory brain systems have been characterized and differentiated by different fMRI techniques and analytical approaches, mainly in the past decade. Frontoparietal regions have been commonly related to working memory, and its crucial role in this cognitive system is perfectly demonstrated by neuroimaging research. However, these brain regions do not work alone; subcortical areas (thalamus, basal ganglia, and cerebellum) permit this working memory function through persistent top-down signals 
from the frontal cortex. Moreover, the importance of dopamine in working memory must also be kept in mind. As D'Esposito and Postle pointed out "the complex interplay of midbrain dopamine in prefrontal and striatal circuits underlies tonic maintenance and phasic gating functions that govern the balance between cognitive flexibility and stability" (D'Esposito and Postle, 2015, p.135). In addition, the temporal lobe may be collaborating with the parietal cortex due to their close connections, and the temporal lobe could be involved in the consolidation of the data maintained in working memory in the long-term memory.

\section{Working memory tasks: $n$-back and PASAT}

Different and varied tasks can be utilized to evaluate working memory capacity. Working memory tasks have evolved from simple span tasks (or memory span task) where participants have to remember short stimulus lists. These new kinds of working memory tasks became known as complex span tasks combining a simple span test (remembering item sequences) with a concurrent processing measure. The first task utilized was reading span, invented by Daneman and Carpenter in 1980. In reading span, between two and six sentences are presented to participants, who have to read them aloud. Then, they have to try to remember the last word in each sentence in order to say all the words aloud in the correct order at the end of the sentences. Other complex span tasks followed, for example, counting span (Case, Kurland, \& Goldberg, 1982), operation span (Turner \& Engle, 1989), rotation span (Shah \& Miyake, 1996), and symmetry span (Kane et al., 2004). Complex span tasks are strongly related to performance on other higher-order cognitive abilities, such as reasoning, reading comprehension, problem solving, mathematics achievement, and intelligence quotient measures (Conway, Kane, \& Engle, 2003; Daneman \& Merikle, 1996; Unsworth \& Engle, 2007). These tasks are also known as 
storage-plus-processing-tests due to their alternation in the recall of some data during an ongoing secondary processing task (Redick \& Lindsey, 2013). For the same reason, they would fall under the category of dual-task tasks. Other tasks that are not included in the dual-task subdivision, such as the Sternberg working memory task, Paced Auditory Serial Addition Test (PASAT), or the $n$-back, have been shown to measure working memory properly (Oberauer et al., 2000). In fact, for years now, the most famous working memory measure is not a dual-task (although a dual version can be made), it is the $n$-back task. $N$ back is thoroughly described in the next section as the most widely used task in neuroimaging research. Currently, the debate about what features a task must have to be a good measure of working memory capacity is a topic of ongoing research.

\subsection{N-back}

The most popular working memory task used extensively in neuropsychological and neuroimaging research is the $n$-back paradigm. In 1958, Wayne Kirchner introduced this task. Nevertheless, $n$-back did not arrive to the neuroscience research field until 1989 , when Dobbs and Rule reported the use of this task to investigate age effects on working memory. It became popular due to its easy administration and simple analysis compared to other working memory tasks (such as Digits from the Wechsler Adult Intelligence Scales), and it is well-adapted to the methodological constraints (stimulus and response timing, response formats) of many neuroimaging techniques because it does not require speech responses or auditory stimuli (Redick \& Lindsey, 2013; Sweet, 2011). Indeed, the $n$-back is one of the most common experimental paradigms for fMRI studies of working memory (Dobbs \& Rule, 1989; Jaeggi, Buschkuehl, Perrig, \& Meier, 2010; Owen et al., 2005; Redick \& Lindsey, 2013; Wager \& Smith, 2003). The current validity of $n$-back has been questioned in some studies that have reported low correlations with other 
working memory tasks (Miller, Price, Okun, Montijo, \& Bowers, 2009; Redick \& Lindsey, 2013) although a recent review provides evidence in the opposite direction (Soveri et al., 2017).

On the $n$-back task, a series of items are presented in succession, and participants have to respond to each stimulus by deciding if it is the same as the stimulus presented " $n$ " items before. For example, on a 3-back task, if the stimuli presented are the letters M B P S N $\mathrm{P} \mathrm{R}$, the examinee has to respond negatively to all the letters except the second $\mathrm{P}$, which would be a 3-back item. The processing load can be varied by manipulating the level of " $n$ ", making the task more or less difficult. Normally, researchers utilize a range from 1to 3-back using 0-back as a control task. In the control part of the task, participants have to respond positively when a predetermined target appears. In fMRI research, blocks are commonly used to present the items on a computer, alternating one or more $n$-back levels with the 0-back as baseline. Multiple versions of the $n$-back task have been designed to measure verbal, spatial, or visual working memory. Normally, the stimuli are presented visually, although auditory items are also seen in some investigations. Studies can include any kind of stimuli: letters (the most popular), images, faces, figures, words, numbers, photographs... (Figure 9) (Sweet, 2011). A variation of the $n$-back task that has recently been utilized in research is the dual version of the task (Jaeggi et al., 2003), where two independent sequences with different $n$-back modalities are presented (usually an auditory $n$-back using letters and a visual-spatial $n$-back using different squares positions). Correct responses numbers and reaction times (RTs) are the behavioral measures that are usually collected by the investigators to check the efficiency.

Different processes are involved in the $n$-back task. To successfully perform the task, it requires selecting stimuli and decision making, encoding the incoming stimuli, 
monitoring, maintenance, updating the buffer, manipulating recalled information, inhibiting inadequate responses, and responding (Jaeggi et al., 2010a; Sweet, 2011). All these processes have to work simultaneously. Therefore, $n$-back is a highly demanding task that involves working memory. In addition, $n$-back is the most widely used working memory task in working memory studies and cognitive training studies. This task has an easy administration that facilitates its implementation in scientific studies; indeed, it is the most frequently used task in neuroimaging. Several meta-analyses of behavioral and neuroimaging studies have been carried out examining the task and its effects on the brain of adults and children (Owen et al., 2005; Redick \& Lindsey, 2013; Soveri, Antfolk, Karlsson, Salo, \& Laine, 2017; Yaple \& Arsalidou, 2018). These meta-analyses of neuroimaging studies with $n$-back studies demonstrate the consistent activation of a widespread frontoparietal network and other cerebral areas. In particular, twenty-four fMRI studies with healthy subjects who performed the $n$-back task were analyzed in the meta-analysis by Owen et al. (2005) in order to find the cerebral regions involved. They studied the brain areas activated depending on the type of stimulus used in the task. For verbal stimuli, the meta-analysis reported that successful $n$-back performance requires efficient coordination of frontoparietal regions and subcortical areas. Specifically, the DLPFC, the mid-ventrolateral prefrontal cortex, the premotor cortex, the SMA, the ACC, the SPC and the IPL, all in both hemispheres, and subcortically, the medial and lateral cerebellum and thalamus, were activated (Owen et al., 2005; Figure 10). As we have seen in previous sections, all these areas take part in a network that is widely activated in working memory. 
Figure 9: Four examples of $\boldsymbol{n}$-back task using different stimuli, all for visual presentation. (A) Spatial (B) Letters (C) Abstract figures (D) Faces.
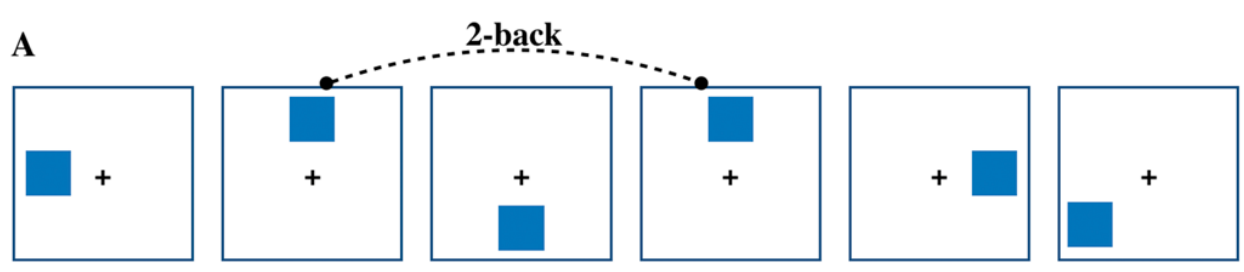

B
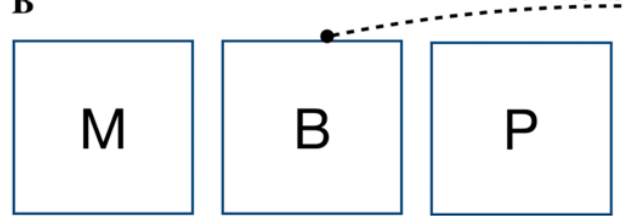

3-back

C
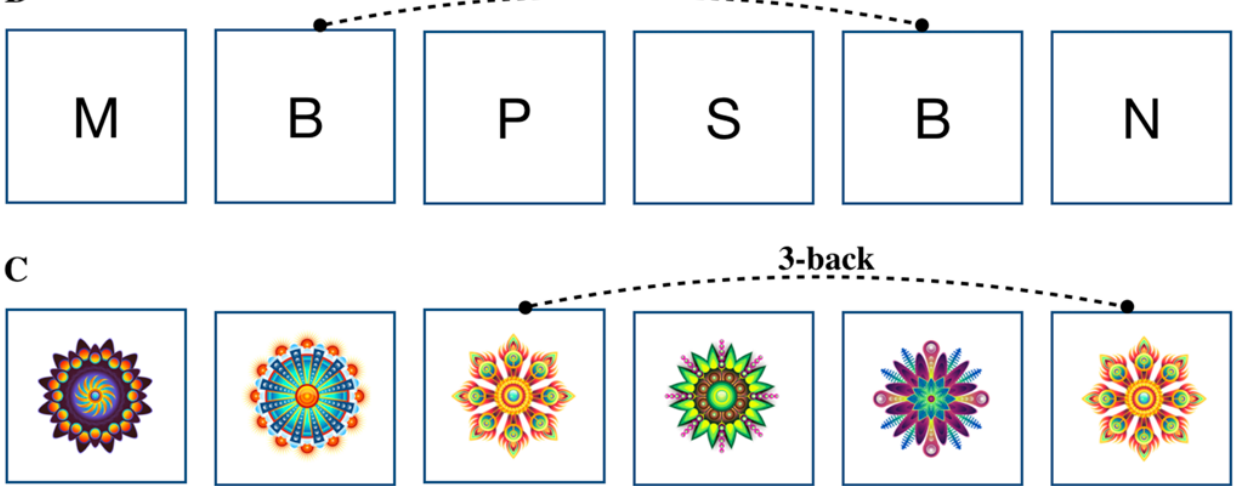

3-back
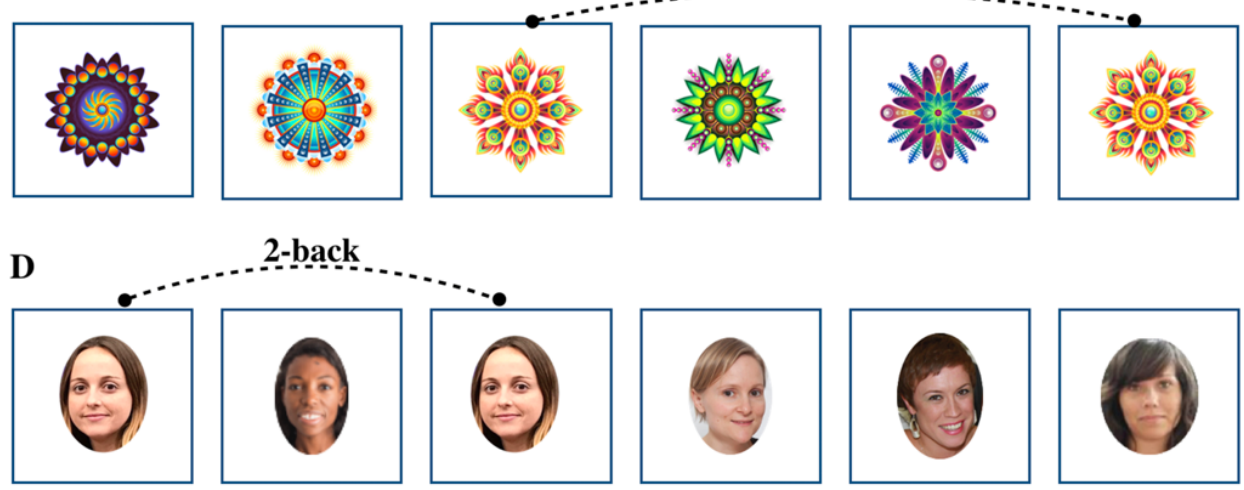

Figure 10: Meta-analytic activation maps for 24 -back studies; bilateral frontoparietal activation can be observed. Extracted and adapted from a meta-analysis focused on the study of the neural correlates of $n$-back performed by Owen et al. (2005, p. 51, Figure 1).

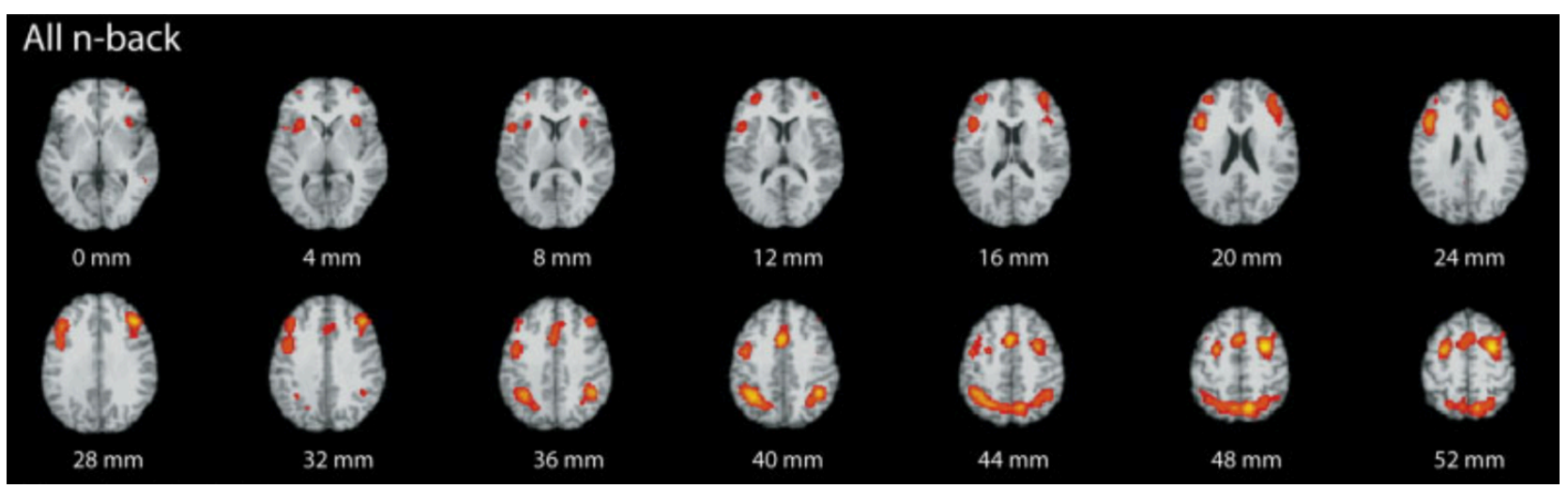


The PASAT (Paced Auditory Serial Addition Test) is a serial addition task designed to evaluate information processing speed, divided attention, and working memory functions (Correia, 2011). This task was originally developed by Sampson in 1956 using verbal and visual stimulus. However, it was Gronwall, in 1977, who started to use the auditive version, although this task has undergone various revisions since Gronwall's publication. Participants who perform the PASAT task are instructed to add each new number they hear (a random single-digit number from 1 to 9 ) to the one immediately preceding it, and say the answer aloud (Figure 11). Normally, the interstimulus intervals last 3 seconds, but in order to increase the cognitive demands, they can be reduced to $2.4,2,1.6$ seconds, or even to 1 second. Diverse versions with different administration times have been developed based on Gronwall's original version. Auditory versions are prerecorded and administrated by a computer, where the participant's answers are recorded. The number of correct responses is the most commonly used score, but computerized versions can provide the reaction times. Subjects have to comprehend the stimuli, respond verbally, attend to the next stimuli, and correctly add it to the previous one, while resisting any urge to add it to their previous answer (Correia, 2011). PASAT execution is related to a significant increase in anxiety, in both healthy and clinical populations. This fact can reduce participants' accuracy and increase misleading answers; as a result, omissions are more common than mistakes. Neuropsychological studies report that performed repeatedly, this task improves performance even over long periods (Tombaugh, 2006). Although the PASAT has been used widely in clinical research (above all with multiple sclerosis patients) it is not uncommon to find it in studies with a healthy population. 
Figure 11: Example of the PASAT task. Participants have to add each new number they hear to the one immediately preceding it and say the answer aloud. The second number is added to the first, the third to the second, and so on.

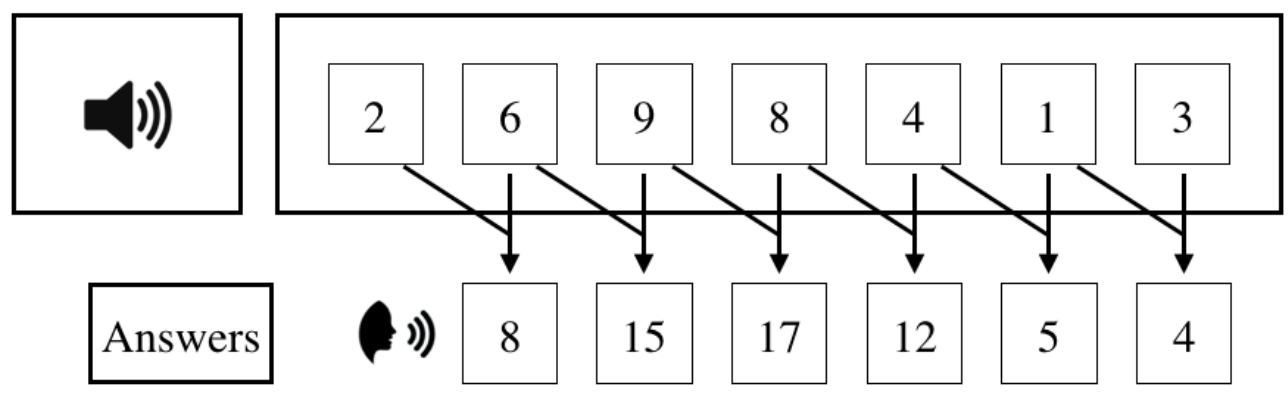

Regarding brain areas related to the PASAT task, fMRI in healthy controls have shown task-related activations in areas related to working memory, that is, frontal and parietal areas. Specifically, cortical activations have appeared in the SMA, the left prefrontal cortex, the bilateral ACC, the left IPC, the left intra parietal sulcus, the right precuneus, the bilateral extrastriate visual areas, the left fusiform gyrus, and the left temporal pole. Regarding subcortical areas, functional activation involves the left cerebellum, the right thalamus, the right caudate nucleus, and the protuberance (Audoin et al., 2005; Figure 12). As mentioned above, this task demands the working memory processes due to the need to maintain and manipulate information in an active way for a few seconds. Participants also have to verbalize the answers. Thus, the intervention in prefrontal parts of the brain would be related to the executive processes of working memory: monitoring and manipulating numbers. In addition, the ACC plays a role in the inhibition of preprogramed responses, and the SMA had been involved in inner speech. In relation to parietal areas, this region has been linked to simple calculation and arithmetical procedures. During the PASAT, the temporal lobe may enable recovery of the learned answers of the simple additions from the semantic memory. Finally, subcortical areas would help by collaborating with the rest of the areas, as explained in preceding paragraphs. 
Figure 12: Brain activation during the performance of PASAT in healthy controls. Extracted and adapted from a work focused on the study of the neural correlates of PASAT in normal subjects by fMRI made by Audoin et al. (2005, p. 98, Figure 1).

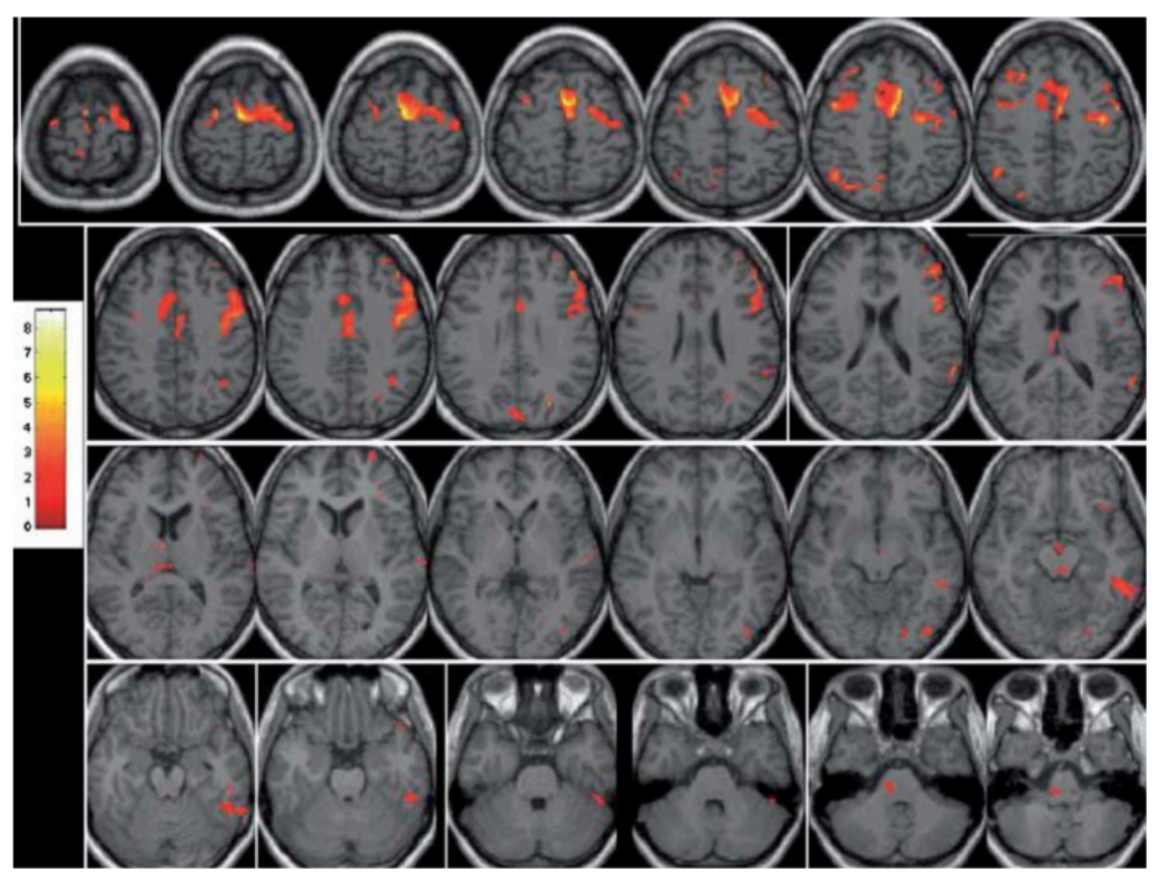

\section{Brain plasticity, automaticity and cognitive training}

The human brain shows remarkable plasticity. Brain plasticity refers to the brain's capacity to change its structure and function to adapt to environment changes, including environment enrichment, sensory deprivation, or increases in sensory stimulations, experiences, and learning (Chang, 2014; Constantinidis \& Klingberg, 2016). Plasticity is not a sporadic phenomenon of the brain, but rather these cerebral changes can occur at any given time throughout the lifespan, from childhood to late in life (Jäncke, 2009; Pascual-Leone, Amedi, Fregni, \& Merabet, 2005). In the human brain, plasticity allows a brain reorganization that may be observable in behavior, anatomy, and function (Chang, 2014; Kelly \& Garavan, 2005; Kleim et al., 2006). In the past few years, fMRI and structural research have provided evidence of brain plasticity (Pascual-Leone et al., 2005). According to Constantinidis and Klingberg (2016), the mechanisms underlying 
plasticity are the activation and suppression of genes, changes in the robustness of synapses, and modifications in the number of dendritic spines, the arborization of the axon, myelination. These authors also pointed out that this process allows the encoding of memories of episodes and places, and knowledge about novel habits and motor skills.

Automaticity is defined as the activities that humans are able to perform without thinking, awareness, or effort. In 1890, William James already described automatic behavior as the actions that are conducted quickly, effortlessly, without dedicating much thinking, and almost performed with no conscious awareness (James, 1890). This process has been explained by classic theoretical psychological frameworks in terms of its characteristics and the psychological processes that should occur when a person is doing an activity under automatic processing (Shiffrin \& Schneider, 1977; Posner \& Snyder, 1975; Bargh, 1994). Thus, these views of automaticity agree that automatic and non-automatic (frequently, called controlled processing) diverge in their descriptive characteristics. In addition, other theoretical conceptions have referred to the development of automatic function, focused on the conditions or processes needed to develop it (e.g., Logan, 1988; Spelke, Hirst, \& Neisser, 1976). Moors (2016) recently reviewed the concept of automaticity. He understands the characteristics of automaticity in terms of factors, such as the amount of attention and time needed to successfully finish an action, with the aim of determining how they interact with the underlying procedure of automatic behavior. In his view, he includes manifold levels of organization of factors in relation to the information, the person and their possible incorporation, considering that this proposal goes beyond the difference between bottom-up and top-down information processing, respectively linked to controlled and automatic processing (Moors, 2016). Thus, 
automaticity allows us to perform activities without thinking about them, which gives us greater freedom to focus on other issues and keeps us from having our minds overloaded.

Cognitive training can be defined as the action of exercising these brain processes by means of specific games or tasks in order to maintain or improve them. This would be like the physical training (cardio or tone up) that improves the body and fitness (Simons et al., 2016). Specialists (i.e. neuropsychologists, occupational therapists) utilize cognitive training as a treatment technique to assist persons who have been neurologically affected by, for example, an illness or a stroke, with one or more of their cognitive processes altered.

After all these definitions, one can say that cognitive training leads to automaticity thanks to the brain's plasticity capacity. As the cognitive practice progresses (e.g. when we learn to read), neural modifications are produced that allow us to act and respond to our environment in an increasingly faster and more unconscious way. Otherwise, people never would be fluent in a second language, for example, or we would always play a videogame like on the first day. A large number of behavioral studies have investigated the utility and impact of cognitive and physical training (for meta-analyses, see Hindin \& Zelinski, 2011; Karbach \& Verhaeghen, 2014; Karr, Areshenkoff, Rast, \& GarciaBarrera, 2014; Kelly et al., 2014). Moreover, neuroimaging research has demonstrated brain changes after cognitive and physical training. Normally, these changes should take place in structures related to the task being trained.

On the one hand, studies have showed training-related structure size brain changes. For instance, Maguire et al. (2000) observed increased gray matter volume in the 
hippocampus in a group of licensed London taxi drivers who had studied to become licensed for an average of two years. The posterior hippocampus of the taxi drivers was significantly larger than in control subjects (Maguire et al., 2000). Other studies have reported gray matter volume changes due to training in relation to, for example, physical skills (juggling) (Draganski et al., 2004), language (Golestani \& Pallier, 2007; Golestani, Paus, \& Zatorre, 2002; Golestani \& Zatorre, 2004; Ilg et al., 2008; Mårtensson et al., 2012; Stein et al., 2012), memory (Draganski et al., 2006; Engvig et al., 2010), and music skills (Bengtsson et al., 2005; Gaser \& Schlaug, 2003a \& 2003b; Hyde et al., 2009; Palomar-García, Zatorre, Ventura-Campos, Bueichekú, \& Ávila, 2017; Sluming et al., 2002). On the other hand, functional changes in the brain have been observed after cognitive training. It has been proposed that the brain areas that are involved in the trained task are those susceptible to change (Buschkuehl, Jaeggi, \& Jonides, 2012; Kelly \& Garavan, 2005; Kelly \& Castellanos, 2014). Different effects have been found in terms of functional changes: cognitive or motor practice may cause increased activity, decreased activity, or both decreases and increases (i.e., redistribution processes) in brain areas related to the trained task or recruiting different areas than those recruited during novel task execution (i.e., reorganization processes) (Buschkuehl, Jaeggi and Jonides, 2012; Kelly \& Garavan, 2005; Kelly, Foxe, \& Garavan, 2006; Poldrack, 2000). Usually, increased activation of common sensorimotor areas has been observed after sensory and motor tasks (e.g., Debaere, Wenderoth, Sunaert, Van Hecke, \& Swinnen, 2004; Münte, Altenmüller, \& Jäncke, 2002; Ungerleider, 2002; Waldschmidt \& Ashby, 2011). Nevertheless, decreased activation appears when the trained tasks require high-order cognitive functions during their performance in the set of cerebral regions that specifically support the task (e.g., Garavan, Kelley, Rosen, Rao, \& Stein, 2000; Hempel et al., 2004; Jansma, Ramsey, Slagter, \& Kahn, 2001; Schneiders, Opitz, Krick, \& Mecklinger, 2011). 
Moreover, training leads to more recruitment of task-specific regions and less reliance on common attention and control regions such as the prefrontal cortex, ACC, and posterior parietal cortex (Kelly \& Garavan, 2005).

\subsection{Working memory training}

In the past, working memory was defined as a rigid attribute, but it is now known that working memory can be improved when adequate training programs are used (Klingberg, 2010; Morrison \& Chein, 2011; von Bastian \& Oberauer, 2014). Currently, a single search in PubMed introducing the words "working memory training" will show more than 2900 studies in the past five years. The cognitive training literature has seen an explosion of interest in working memory training in the last few years, accumulating a growing number of studies that evaluate its effect. Given this strong interest and the variety of the results, a number of meta-analyses have been carried out in the past five years $(\mathrm{Au}$, Buschkuehl, Duncan, \& Jaeggi, 2015; Au, Sheehan, et al., 2015; Dougherty, Hamovitz, \& Tidwell, 2016; Melby-Lervåg \& Hulme, 2013, 2016; Melby-Lervåg, Redick, \& Hulme, 2016; Schwaighofer, Fischer, \& Bühner, 2015; Soveri et al., 2017; Weicker, Villringer, \& Thöne-Otto, 2016). As a result, all these studies have a variety of training programs, working memory tasks, participant groups, and experimental designs. The training time can vary from minutes to hours, and the number of sessions from one day to months. Complex span, $n$-back, mental calculation, or memory updating are some of the many working memory tasks that researchers use for their training. The training task, in turn, can be single or dual, and the training paradigm can be adaptive or not. The participants who train can be investigated alone, compared to a passive control group with no training, compared to an active control group that trains the same amount of time on a non-working memory task, or compared to both classes of control groups. Soveri and colleagues, in 
their meta-analysis, investigated all these issues and did not find significantly different training effects of the type of control group, the training type, the number of sessions, or the hours of training. However, it should be noted that they only examined studies that used $n$-back as a training task.

A large number of studies have been conducted on cognitive training using $n$-back as the main task. All of them suggest that adequate $n$-back training improves task performance in terms of accuracy and reaction times, even with relatively short-term training (e.g. Buschkuehl et al., 2014; Hempel et al., 2004; Jaeggi, Buschkuehl, Jonides, \& Perrig, 2008; Küper \& Karbach, 2016; Li et al., 2008; Salminen, Strobach, \& Schubert, 2012; Schneiders et al., 2011; Takeuchi et al., 2010; Thompson et al., 2016; Yamashita, Kawato, \& Imamizu, 2015). Generally, participants double or triple their pre-training performance levels (Jaeggi et al., 2008; Kundu et al., 2013; Redick \& Lindsey, 2013; Thompson et al., 2013). The simple way of responding to stimuli and the easy management of difficulty makes the $n$-back task an appropriate tool to monitor working memory processes. The majority utilized an $n$-back adaptive task during training to manipulate the level of difficulty depending on the participant's performance. The researchers used training programs ranging from 60 to 1500 minutes, with no major differences in improvement (Anguera et al., 2012; Jaeggi et al., 2010b; Schneiders et al., 2012; Vartanian et al., 2013), but little research has focused on the effects of brief $n$-back training. Despite all the existing literature, only a few studies have tested the long-term (2-8 months) effects of $n$ back training, and they found that the behavioral changes observed remained stable (Jaeggi, Buschkuehl, Jonides, \& Shah, 2011; Jaeggi, Buschkuehl, Shah, \& Jonides, 2014; Katz et al., 2017; Li et al., 2008). Both the single $n$-back and the dual $n$-back task have been used in training studies, with the latter being the most widely utilized, although both 
have shown efficacy in improving working memory capacity (Jaeggi et al., 2010b). In a very recent study, Küper \& Karbach (2016) compared brief single and dual $n$-back training, concluding that both showed equivalent behavioral improvements. Moreover, the authors concluded that, in short periods of training, single $n$-back training can be more effective than dual $n$-back training (Küper \& Karbach, 2016). These authors added to others that have shown that both single and dual $n$-back have been effective in producing training effects (Soveri et al., 2017).

\subsubsection{Brain effects of working memory training on monkeys}

Animal models are necessary for behavioral paradigms and well-chosen neuropsychological studies in order to clarify the neural bases that support working memory. Despite the fact that animal studies involve distinct behavioral and task conditions, they use comparable definitions of working memory with human studies (Funahasi, 2017). Electrophysiological studies with monkeys have been used to investigate the cellular basis of working memory while they are performing a delayedresponse task (remembering the spatial location or identifying a stimulus over a delay period of a few seconds). Neural connections between the prefrontal cortex and posterior parietal and inferior temporal cortex and subcortical structures (including basal ganglia and the thalamus) are present during the delay period of working memory tasks, as are a network of interconnected neurons in the same prefrontal cortex (Constantinidis and Klingberg, 2016). Lesion experiments in macaques have confirmed that the DLPFC plays a crucial role in delayed-response performance (Funahasi, 2017). Working memory training has been studied in non-human primates as well. These investigations are consistent with the results of human training imaging studies. Increased activity was found in the prefrontal cortex after training in the delay period of the task, above all in 
the ventrolateral prefrontal cortex. In spite of this increase, decreased activity has also been found. After training, the average neuronal selectivity for a stimulus decreases. Constantinidis and Klingberg tried to explain this phenomenon in a specific way: "a probable explanation of the decrease in neuronal selectivity for the trained stimuli is that a large portion of neuronal activity after training represents factors that are not specific to the stimuli, but that are related to the rules and execution of the working memory task, such that the activity that is selective for the trained stimuli declines as a percentage of total neuronal activity" (Constantinidis and Klingberg, 2016, p. 6).

\subsubsection{Brain effects of working memory training on humans}

Few studies have examined the cerebral changes produced by cognitive training in working memory, and most of them have focused on functional changes during the performance of working memory tasks before and after the training. Buschkuehl and colleagues reviewed these studies and concluded that there was evidence for brain changes in specific areas in terms of activation, but, as mentioned above, there was no agreement about whether this activation increased, decreased, or underwent redistribution, or even whether a reorganization of networks took place (Buschkuehl et al., 2012). The most common outcome is functional changes in the activity of those areas that are already activated during the task before practicing. In the case of working memory training, these training-related changes are normally located in frontoparietal regions (Figure 13). Other areas, such as the MTL, have been less involved in the training effect. However, subcortical regions, such as the striatum, have been related to changes after practice, although striatal changes are not as consistent a finding as frontoparietal changes (Constantinidis and Klingberg, 2016). 
Figure 13: Brief abstract of elements underlying working memory training-related increases in capacity. Extracted and adapted from Constantinidis and Klingberg (2016, p. 10, Figure 3). "Studies using fMRI and electro-encephalography in humans associate stronger frontoparietal functional connectivity and structural connectivity with higher capacity, and training studies show increased frontoparietal connectivity after working memory training. Functional connectivity could be related to either myelination or stronger synaptic connectivity. fMRI in humans has consistently shown changes (both increases and decreases) in brain activity in frontal and parietal regions after training. Simulations using neuronal networks suggest that the capacity of a stimulus-encoding network can be increased by stronger functional connectivity between the prefrontal cortex and parietal cortex. Genetic studies have associated greater training improvement with certain polymorphisms of the postsynaptic dopamine D2 receptor gene (DRD2) and the presynaptic sodium-dependent dopamine transporter gene (DAT1) both of which are highly expressed in the striatum. fMRI in humans suggests that the striatum mediates the effects of working memory training. Positron-emission tomography studies have detected changes in striatal dopamine release and cortical dopamine D1 receptor density. MFG, middle frontal gyrus; PPC, posterior parietal cortex; SFG, superior frontal gyrus."

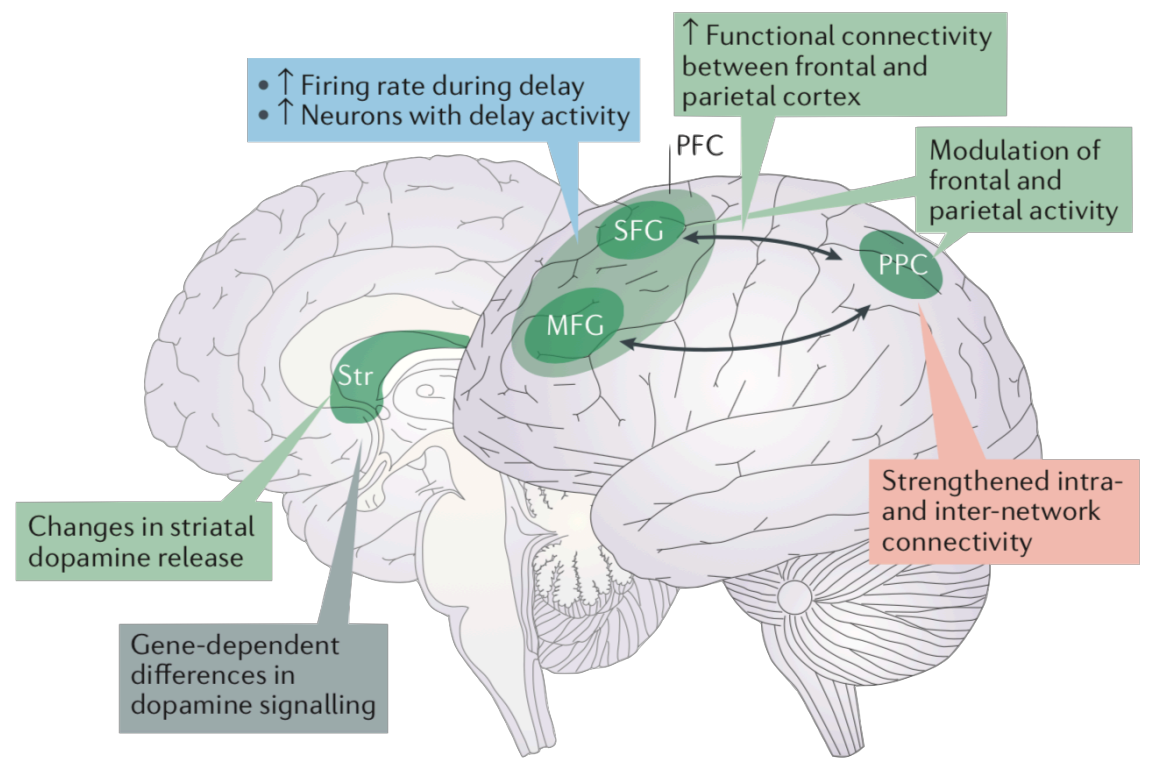

Less evidence has been found for increases in brain activation after working memory training compared to reports of decreases. For example, Westerberg \& Klingberg, (2007) evaluated the cerebral changes in only three young volunteers after five weeks of working memory training, and they found a significant increased activation in the middle or IFG and parietal cortex. This finding coincides with the results from a similar prior experiment 
carried out by this group (Olesen, Westerberg, \& Klingberg, 2004), where they found increased brain activity in the middle frontal gyrus and superior and inferior parietal cortices after five weeks of cognitive training. Second, regarding research that observed both increased and decreased activation (activation redistribution) after working memory training, one study by Dahlin et al. (2008; Experiment 1) stands out. In this study, analyses of pre- and post-training changes in the fMRI data showed increased activation in the left striatum, temporal, and occipital regions, but also decreased activity in frontal and parietal areas. Participants engaged in five weeks of computer-based updating training on a working memory task. According to Constantinidis and Klingberg (2016), an increase in cerebral activation may reflect a higher firing rate during the delay or an increase in the number of cells with delay activity; and some computational studies relate higher capacity to increased brain activity.

The majority of fMRI studies that reported a decrease in cerebral activation used the $n$ back task for training, and all the studies agree on the brain regions where the activation reductions were found (Schneiders et al., 2011, 2012; Schweizer, Grahn, Hampshire, Mobbs, \& Dalgleish, 2013; Thompson et al., 2016). Schneiders et al. (2011) used an 810 day adaptive $n$-back training (between 400-500 minutes) and performed fMRI pretest and posttest sessions. The authors observed decreased activation in the right superior middle frontal gyrus (BA 6/9/46) and right posterior parietal lobule (BA 40). In the same vein, Schneiders et al. (2012) reported the same activation pattern after training their participants for approximately the same length of time as in their prior study, but only on an adaptive auditory $n$-back task. Another study that used $n$-back for training was by Schweizer et al. (2013), where the researchers trained their subjects for between 20-30 minutes a day for 20 days. They found activation decreases in the 3-back load level in the left DLPFC, right superior frontal gyrus, bilateral supramarginal gyrus, bilateral middle 
temporal gyrus, and bilateral middle occipital lobe. In other work, participants were trained on an adaptive dual $n$-back task in 20 sessions distributed across 4 weeks. Before and after the training, volunteers were scanned on the non-adaptive trained task. Once more, they reported reductions in activation in the bilateral inferior and middle frontal gyrus, insular cortex, and intraparietal sulcus (Thompson et al. 2016). The last example, a study carried out by Vartanian et al. (2013), showed that very brief working memory training also causes cerebral modifications. They performed only 60 minutes of single $n$ back training and reported decreased brain activation in prefrontal areas (BA 46/47) (Vartanian et al., 2013).

Several researchers have interpreted decreases in the activation of cerebral regions as an indicator of better neural efficiency of the area in performing its function, which means that less cerebral resources are needed to give an appropriate response to the task after practicing. This decline in cerebral activation may allow participants to respond more quickly and make fewer mistakes (Buschkuehl et al., 2014). Kelly, Foxe, \& Garavan (2006) noted that this effect of decreased activation is typically observed after training on higher cognitive tasks, and they stated that lower activation is associated with increased neural efficiency, which means that fewer neurons are needed to give a fast and accurate answer to the task. However, some studies have criticized the better neural efficiency explanation for the decreases in activation for being overly simple and unclear (Constantinidis \& Klingberg, 2016; Poldrack, 2015). In his review, Poldrack (2015) viewed efficiency as inverted energy for the transmission of information in the brain networks. He highlighted the need for new studies and models to examine the neural changes, and he reported that identifying potential activation effects may lead to future mechanistic explanations. 
One of the most important cellular mechanisms of plasticity is the change in connectivity while the brain is performing an activity and when it is doing not a single task, that is, at rest. At the same time, differences in functional connectivity may be associated with individual differences in working memory capacity. Despite this importance, there has been little research published with respect to connectivity changes after working memory practice. Some of this literature, which investigated resting-state connectivity after working memory training using fMRI, showed increased frontoparietal network connectivity after training, and it positively correlated with the performance on the trained task (Jolles, van Buchem, Crone, \& Rombouts, 2011; Takeuchi et al., 2013). Evidence from a magnetoencephalography study with children found increased functional connectivity related to training-induced improvements in working memory capacity as shown by the positive correlation between frontoparietal connectivity and improvement on the task (Astle et al., 2015).

In addition, EEG and fMRI studies that investigated functional connectivity during the task in adults and children showed that the connectivity between frontal and parietal areas also increased and correlated with behavioral performance improvements (Kundu et al., 2013; Thompson et al., 2016). These results tell us that stronger connections between frontoparietal regions play a role in improving working memory. A stronger synaptic connectivity between neurons or an activity-dependent increase in the myelination of the connecting axons may be the mechanisms underlying functional connectivity changes (Constantinidis \& Klingberg, 2016; Gibson et al., 2014; Yeung et al., 2014).

In the same vein, scarce research has been done about structural changes, that is, gray and white matter changes, after working memory training. In a review of working memory training studies by Takeuchi, Taki, \& Kawashima (2010), working memory capacity 
correlated with regional gray matter and withe matter volume in frontal and parietal regions. After that, Takeuchi and colleagues used voxel-based morphometry (VBM) to investigate structural changes in the brain after about 20 hours of working memory training. After all this specific training, they found decreases in gray matter volume in the bilateral DLPFC, bilateral parietal lobe, and left superior temporal gyrus (Figure 14; Takeuchi et al., 2011). Other works studied the gray matter in a different way (Caeyenberghs, Metzler-Baddeley, Foley, \& Jones, 2016; Román et al., 2016). They studied the gray matter indices of cortical thickness and cortical surface area using different techniques such as surface-based morphometry. Both studies performed a long working memory training ( 2 and 3 months) using distinct tasks, and they reported different results. Metzler-Baddeley and colleagues found an enlargement of cortical thickness in the right caudal middle frontal cortex and in the left pallidum after training. On the other hand, Román et al. (2016) reported that, after 3 months of $n$-back training, the training group showed conservation of gray matter compared to the control group in the middle temporal, ventral frontal, inferior parietal cortices, and pars opercularis.

Diffusion tensor imaging (DTI) was the technique used by the researchers to study the white matter changes in the brain after working memory training. DTI is a MR-based technique that yields measures of the magnitude and direction of water diffusion in human brain tissue. To measure the directionality of diffusion and quantify free diffusion of water within a voxel, fractional anisotropy (FA) is used. Anatomical characteristics of white matter (axonal membrane thickness and diameter, fiber density and myelinization) are reflected by the magnitude of FA (Buschkuehl et al., 2012). Therefore, increases in FA would reflect stronger anatomical connections. That said, to our knowledge only two studies have investigated white matter changes after working memory training. These 
DTI studies observed that working memory training increases the FA in frontoparietal, occipital and temporal, occipital and frontal and left and right frontal areas in the trained group (Takeuchi et al., 2010; Salminen, Martensson, Schubert and Kühn, 2016).

Figure 14: Changes in gray matter volume after working memory training found by Takeuchi et al. (2011, p. 6, Figure 2).
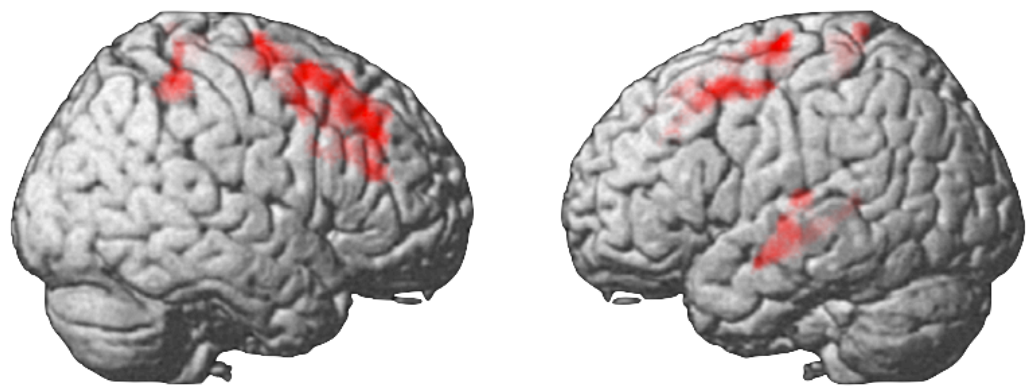

Dopaminergic transmission has an important role in higher cognitive functions and in working memory, as we have seen in previous sections. So, are there dopamine-related changes after a working memory training? Once more, there are few studies assessing this question, but there is evidence that the dopaminergic system and the striatum play a crucial role in the improvement in working memory after training (Buschkuehl et al., 2012; Constantinidis \& Klingberg, 2016; Takeuchi et al., 2015). Dopamine has different implications in working memory training. There would be individual differences in improvements after training depending on the variability in dopaminergic signaledrelated genes, as pointed out in diverse genetic studies (Brehmer et al., 2009; Söderqvist et al., 2012). In addition, PET studies in humans pointed out that dopamine release and dopamine receptors change due to working memory training (Backman et al., 2011; McNab et al., 2009), coinciding with the results in experiments with animals (Wass et al., 2013). Computational studies suggest that the dopaminergic system improves the capacity to retain information in working memory despite distractors (Durstewitz, 
Seamans, \& Sejnowski, 2000b, 2000a). Constantinidis and Klingberg (2016) conclude that, given all this evidence, it would be useful to investigate new pharmacological interventions affecting the dopaminergic system.

All these cerebral changes produced by cognitive training that have been investigated were studied only in the short-term, and so the stability over time of neural changes produced by this training remains unknown.

\subsection{The controversy of working memory training: transfer effects}

As is well known, the effects of cognitive training have become a popular research issue. Specifically, in recent years, working memory training has been studied by a large number of researchers, but with no agreement reached. We have seen that working memory training shows improvements on the trained tasks (see e.g. Klingberg, 2010; Morrison \& Chein, 2011; von Bastian \& Oberauer, 2014 for reviews). The question that is still controversial is whether working memory training has transfer effects to other untrained tasks. Literature has shown a relationship between working memory capacity and several cognitive processes, for instance, fluid intelligence (Gf) (von Bastian \& Oberauer, 2014). There are two types of transfer effects that can occur: near transfer, when the trained task is similar or identical to the transfer task, and far transfer, which occurs when the transfer measures differed substantially from those trained, i.e. nonverbal ability, verbal ability, reading comprehension, word decoding, or arithmetic (MelbyLervåg et al., 2016). As mentioned above, there is no agreement about behavioral reports: some studies have reported near and far transfer (Buschkuehl et al., 2014; Jaeggi et al., 2011; Jaeggi, Studer-Luethi, et al., 2010; Schweizer et al., 2013), others only near transfer (Harrison et al., 2013; Minear et al., 2016; Salminen et al., 2012; Waris, Soveri, \& Laine, 
2015), and others did not find any kind of transfer (Chooi \& Thompson, 2012; Clark, Lawlor-Savage, \& Goghari, 2017b; Redick et al., 2013; Thompson et al., 2013).

Given this controversy, a number of meta-analyses have recently been carried out to examine the efficacy of working memory training (Au, Buschkuehl, Duncan, \& Jaeggi, 2015; Au, Sheehan, et al., 2015; Melby-Lervåg \& Hulme, 2016; Melby-Lervåg, Redick, \& Hulme, 2016; Soveri et al., 2017). On the one hand, Au et al. $(2015,2016)$ found support for near and far transfer effects, whereas Melby-Lervåg et al. $(2015,2016)$ found moderate near transfer effects, but they concluded that there are no convincing effects of far transfer. In addition, Soveri et al. (2017) reported a moderate effect of task-specific transfer, but very small near and far transfer effects. All these meta-analyses examined studies of working memory training that used the $n$-back task in their training paradigms. The contention continues when it comes to deciding on the training design. How many hours of training? How many sessions? Should we use an adaptive training? What kind of control group should we use? In the aforementioned meta-analysis, Soveri et al. (2017) did not find significantly different training effects of the type of control group, the training type, the number of sessions, or the hours of training.

Despite the vast number of behavioral studies, as explained above, there are few studies examining the cerebral changes produced by working memory training (see the subsection 5.1.2 Brain effects of working memory training for detailed information). The research that focused on the study of the transfer effects in the brain is even more scarce. One aforementioned study by Dahlin et al. (2008; Experiment 1) tried to confirm the hypothesis that transfer will occur if the training and the transfer task involve, at least in part, the same brain regions and processing components. In that study, participants 
engaged in five weeks of computer-based updating training on a working memory task (i.e. test of letter memory). $N$-back and Stroop tasks were included as transfer tasks, even though the Stroop is not a working memory task. The letter memory task and the $n$-back task are two similar tasks that differed with regard to stimuli and rules. Analyses of posttraining changes in the fMRI data during the performance of the letter memory task showed increased activation in the left striatum, temporal, and occipital regions, but also decreased activity in frontal and parietal areas. The near transfer effects were seen in left striatum and frontal cortex, with increased activation, and no significant changes for far transfer were found. The authors confirmed the hypothesis that transfer will occur if the training and the transfer task involve the same brain regions and processing components. Following this study, Salminen, Kühn, Frensch and Schubert (2016) wanted to test this hypothesis. However, they presented results that support the idea that $n$-back training did not improve general cognitive resources, but rather a specific cognitive process of WM updating. In this study, participants performed an adaptive dual $n$-back training (sixteen 30-minute training sessions) and compared them to an active control group that did an adaptive single $n$-back training (the same time as the trained group) and a passive control group that did not receive any kind of training. They use a letter memory task (both, dual and single version) as the near transfer task. All the participants took two fMRI sessions (pre and post-training). They reported near transfer behavioral effects only for the trained group from the dual $n$-back task to the letter memory task (in its dual version). Regarding the neuroimaging results, they found decreased activation in the trained group in frontoparietal areas, thalamus, and ACC, but, at the same time, the results yielded increased activation of the striatum and the superior temporal lobe during the trained task. For neural transfer effects during the dual letter memory task, only increases in the occipital cortex and in the striatum were found for the trained group. In light of their 
results, these authors believe that overlap of the frontoparietal network at pretest or a certain pattern of training related to the decrease in this overlap cannot be a critical precondition for having transfer between two tasks. They conclude that to observe transfers effects, they should be associated with increased activation in an updatingprocess specific region, the striatum (Salminen, Kühn, Frensch, \& Schubert, 2016).

In another study (Beatty et al., 2015), the researchers wanted to test this hypothesis again. They used the delayed matching-to-sample task (dMTS) as a near transfer task and the single $n$-back task as a training task. Participants performed three 20-minute training sessions. They had one post-training fMRI session (performing only the dMTS) and compared the activation of the experimental and control groups. Their results showed greater activation in left inferior frontal gyrus, right posterior parietal cortex, and the cerebellum in the training group compared to the control group, only in one phase of the dMTS task. A very recent study (Clark, Lawlor-Savage, \& Goghari, 2017a) investigated the neural effects of cognitive training and transfer after a working memory training $(n$ back). They added evidence to other studies that reported decreased activation in frontoparietal areas after $n$-back training. Regarding the far transfer brain effects, the authors expected reductions in frontoparietal areas while the $\mathrm{G} f$ task was performed, but they did not find them. They confirmed that $n$-back training decreased brain activation on the task, but it did not produce any kind of neuroplastic changes while performing the intelligence task. Another recently released working memory training study compared HIV patients to healthy controls (Chang et al., 2017). The participants trained on an adaptive $n$-back task (20 to 25 sessions in 5-8 weeks) and completed three fMRI sessions (before, 1 month, and 6 months after finishing the training) while performing 1- and 2back tasks. They also performed two near transfer tasks outside of the scanner. Their 
fMRI results showed improvements in performance and, once more, decreased activation in frontoparietal areas in both groups 1 and 6 months after $n$-back training. These brain areas were different depending on the group. Importantly, the decreased activation in the DLPFC during 2-back correlated with improvements in short-term memory (i.e., Digit Span task) in the HIV group, whereas decreases in activation in the middle frontal cortex during 1-back correlated with short-term improvements for both groups. Therefore, the decreased activation in frontal areas in the trained task was related to near transfer. After observing the correlations between the brain changes and the improvements on the near transfer tasks, the authors concluded that working memory training was associated with better neural efficiency that led to improvement on other working memory tasks.

In summary, it is difficult to make predictions about what will happen in the brain after a working memory training, given all this controversy about the transfer effects and the lack of studies on brain transfer effects. There is behavioral evidence that near transfer occurs when the transfer task shares specific cognitive processes with the training task (Salminen et al., 2012; Sprenger et al., 2013), which moves in the direction of the aforementioned hypothesis that transfer will occur if the training and the transfer task involve the same brain regions and processing components. Studies also pointed out that the striatum may have an important role in transfer effects because it mediates the effects of working memory training. In addition, once again, the scarce brain transfers effects that have been investigated were studied only in the short-term, and so the stability of neural transfer changes over time produced by this training remains unknown. 


\subsection{Conclusion}

Multiple studies of working memory have been carried out in recent years, and there is still a long way to go before the mechanisms underlying its cognitive processes and its training can be clarified. Neuroimaging techniques to study the effects of learning and training in the adult brain have been a crucial tool to investigate this. Nevertheless, although there is a consensus about the cerebral regions that operate working memory, training is still controversial. Training effects have been studied at the functional level, but with no agreement reached: activation increased, decreased, underwent redistribution, or a reorganization of networks took place. The same thing is true of the scarce structural findings, where increases and decreases in gray matter volume have been observed. Furthermore, very little research has investigated the functional connectivity changes after working memory training in both a resting state and during the task. Transfer effects have a similar fate because there are opposite results, and studies do not show agreement. Importantly, it must be highlighted that all the investigations studied the training-related effects in the short-term. The stability of these changes has not been researched yet. 


\section{Overview of the experiment}

The present thesis explored the neural and behavioral changes after a working memory training in the short- and long-term using MRI techniques. As pointed out in the previous chapter, a large number of working memory training studies have been carried out. However, these experiments are mostly behavioral, showing the training-related improvements in the performance of the same task when the trained task is similar to the transfer task (near transfer) and when transfer measures differed substantially from those trained (far transfer). The results are controversial, and no agreement has been reached because the results obtained were in opposite directions. It has been widely demonstrated that working memory training causes improvements in the trained tasks (Klingberg, 2010). However, regarding near and far transfer, more evidence has been found for near transfer (e.g. Buschkuehl et al., 2014; Jaeggi et al., 2011; Schweizer et al., 2013; Harrison et al., 2013; Minear et al., 2016; Salminen et al., 2012; Waris, Soveri, \& Laine, 2015) than for far transfer, which has been demonstrated less.

It is well known that cognitive training can improve the trained task when adequate training programs are used (Klingberg, 2010). The vast majority of working memory training studies differ in their experimental designs. Researchers vary their training sessions from one day to months and the total amount of training from minutes to hours, with no major differences in improvement (Soveri et al., 2017). Where there is agreement is regarding the trained task: the $n$-back is the most widely used task in training protocols. 
More extended is the use of the dual version of this popular task for training, but it has been demonstrated that its single version produces similar benefits (Jaeggi et al., 2010b; Küper \& Karbach, 2016; Soveri et al., 2017). Regarding long-term effects, behavioral studies showed that the effects of $n$-back training remain stable after months (2-8) without training (Jaeggi et al., 2011; 2014; Katz et al., 2017; Li et al., 2008). In addition, there is behavioral evidence that near transfer occurs when the transfer task shares specific cognitive processes with the training task (Salminen et al., 2012; Sprenger et al., 2013).

Improvements on the $n$-back training have been extensively demonstrated (Au et al., 2015; Soveri et al., 2017), and they are frequently accompanied by changes in the activation of brain areas related to this task, normally located in frontoparietal regions and in subcortical regions, especially in the striatum (Buschkuehl et al., 2012). The prefrontal cortex has a crucial role in executive functions (maintaining, updating, manipulating, etc.), and it is also important because of its interactions with other brain regions while the working memory processes. The parietal cortex is necessary when an attentionally-demanding upholding task is in progress. In addition, the striatum is a relevant information filter in working memory, and it plays a role in the improvement of working memory after training by facilitating plasticity (Buschkuehl et al., 2012; Constantinidis \& Klingberg, 2016; Takeuchi et al., 2015). Activation increases or decreases after training depend on the training regimen or the training task used. When the $n$-back is the task used for the training, most of the studies agree that the main effect found after training is decreased activation, and the areas where the effects were seen also matched: frontoparietal areas (e.g. Schneiders, Opitz, Krick, \& Mecklinger, 2011; Schneiders et al., 2012; Thompson, Waskom, \& Gabrieli, 2016; Vartanian et al., 2013). 
However, the interpretation of these functional changes is not well understood. It should be recalled that neuroimaging research in this specific field was scarce, and all the investigations studied the training-related effects in the short term. One relevant point is that the stability of these changes has not yet been investigated to show which changes are stable (are the real result of training) and which changes are transient (changes that help to generate stable changes). In the last year, one study carried out with HIV patients performed a follow-up fMRI session 6 months after finishing the training. The results suggest a stability of the effects on the trained task in the left ACC (BA 25, 32), left SFG (BA 8), medial precuneus (BA 7), and left IPL (BA 40) (L. Chang et al., 2017).

In addition, the use of other neuroimaging techniques beyond task fMRI may help to understand these functional changes. One possibility is to investigate structural changes, applying the idea that training may modify the structure of brain areas involved in the task. An increased gray matter volume in the main areas involved in the task should be expected, as indicated by the literature (see Thomas \& Baker, 2013 for a review). However, previous studies reporting these changes have obtained a heterogenous pattern of results (Colom et al., 2016; Román et al., 2016; Takeuchi et al., 2011). Therefore, the study of stable and transient changes may serve to clarify this issue.

Even more scarce are studies to determine which consistent brain changes underlie transfer effects from one trained working memory task to other non-trained task (MelbyLervag and Hulme, 2016). The few existing fMRI studies have pointed to different transfer effects when studying near transfer. One hypothesis postulated that transfer occurs if the training and the transfer task involve the same brain regions and processing components (Dahlin et al., 2008). The analyses of post-training changes in the fMRI data 
in this study showed near transfer effects in the left striatum, which was involved in the trained and untrained task. Other researchers reported similar near transfer brain effects: decreased activation in the trained group in frontoparietal areas, thalamus, and ACC, but at the same time, the results yielded increased activation in the striatum and the superior temporal lobe during the trained task (Salminen et al., 2016). However, another study did not find any type of functional changes while performing the transfer task (Clark et al., 2017a).

fMRI studies have emerged to measure the brain during the performance of tasks related to perception, attention, and high-order cognitive processes, but also to measure the brain during resting-state conditions, where the spontaneous functional activity of the brain is recorded. This method was used because it is one of the different neuroimaging techniques that currently make it possible to measure brain activity during the performance of experimental working memory tasks. The main benefits of the fMRI technique are its non-invasiveness and its ability to take an in-vivo measure of ongoing human processes under a diverse number of conditions. In addition, fMRI techniques have good temporal resolution and stand out in spatial resolution. Thus, they are appropriate for mapping brain activity. Data analysis methods have also evolved, accompanying the evolution of fMRI human data acquisition. Task-activation fMRI analysis is only one approach among many others that are applicable to fMRI data. In other words, fMRI data sets should be studied from many different perspectives, allowing them to better characterize the human brain processes. In this research, three fMRI sessions were conducted; the second fMRI session took place seven days after the first one, and the last one thirty-five days (five weeks) after the second session. On the other hand, VBM is the most widely applied method to investigate the structural changes as a 
function of learning and training in the living brain. VBM enables voxel-wise statistical comparison of local gray matter volume between groups or time-points in the whole brain, including subcortical structures (Thomas \& Baker, 2013).

That being said, the first question posed to begin all this work was: what happens to the training-related changes in the brain after the training is over? Do they remain stable or they will increase to compensate for the lack of practice? Therefore, in the short(immediately after training) and long-term (after five weeks with no training), the behavioral (i.e., improvements in accuracy and reaction times) and fMRI task-activation changes related to $n$-back training effects were investigated. This will also allow it to distinguish between sustained and transient changes due to training. Closely related to the first question, a second one arose: Are there far transfer brain effects when two working memory tasks are structurally different but share cerebral areas and specific processes? Once more, we investigated in the short- and long-term the behavioral and fMRI trainingrelated changes during the performance of the far transfer task. Finally, the last relevant question addressed in this work was: does this specific short n-back training produce structural changes in the brain? Do these changes affect the frontoparietal areas involved in the task? We assume that training should involve changes in the main areas related to the task.

Taking into account the existing literature, we were very clear that $n$-back would be the training task that we would use in our experimental design. As the far transfer task, we decided to choose the PASAT task. These tasks are two of the most common experimental working memory paradigms that continuously require holding information available for subsequent complex processing (Jaeggi et al., 2010a; Owen et al., 2005; Tombaugh, 
2006), and both require the participation of the DLPFC. More, they have the premotor cortex, the dorsal anterior cingulate cortex, and the posterior parietal cortex in common (Bertrand Audoin et al., 2003; Correia, 2011; Forn et al., 2011; Owen et al., 2005). These tasks require the maintenance of past verbal information, but with different posterior cognitive processes involved: the $n$-back task requires maintaining letters, whereas the PASAT requires maintaining a number, resisting the interference of a generated number and adding it to the actual number. Additionally, the PASAT test is fast and easy to administer, and it can also be easily adapted to the specific requirements of the fMRI. Regarding the training program, we decided to hold four consecutive sessions where the trained participants had to complete 200 minutes of single $n$-back in our laboratory located at the University. This specific training is considered short and intensive. Klingberg (2010) recommended two sessions per week during a period of three months. So, why did we choose this particular training? Literature indicated that short training times produced the same behavioral improvements and cerebral changes as long training protocols (Buschkuehl et al., 2014; Jaeggi, et al., 2008; Küper \& Karbach, 2016; Vartanian et al., 2013; Yamashita et al., 2015). In addition, we have chosen a brief single $n$-back training with an eye on future clinical interventions. A long training protocol might be difficult and costly for patients and institutions. Therefore, we wanted to evaluate the effects of this kind of short working memory training regime on healthy controls to facilitate comparisons with clinical populations in future studies. Moreover, one study concluded that, in short training periods, single $n$-back training can be more effective than dual $n$-back training (Küper $\&$ Karbach, 2016).

Due to the aforementioned features of the fMRI technique and the suitability of the $n$ back and PASAT tasks for studying the effects of working memory training, the use of 
this neuroimaging technique and the implementation of both tasks seemed to be a plausible strategy to address these questions. The methodological approach used was task-fMRI. This technique was utilized to investigate the specific brain areas involved in working memory (in both tasks), and then study the effects that a brief and intensive $n$ back training would have immediately and five weeks later with no training. In addition, VBM was the methodological approach selected to study the gray matter structural changes. With this approach, gray matter volume changes can be easily studied after the $n$-back training, also investigating their relationship with the improvements on the working memory tasks used in the present study.

All things considered, this work would be the first working memory training study to investigate the stability of cerebral changes, after weeks with no training, on the trained task, the transfer task, and the gray matter volume in control subjects. Additionally, the present thesis would contribute more behavioral and neural empirical data to the cognitive training field, above all to the neuroimaging research, where the results are still unclear. Moreover, studying the cerebral mechanisms supporting the behavioral effects will contribute empirical neurobiological evidence to the theoretical models whose aim is to describe the working memory training system. In order to complete the objectives of this thesis, the fMRI technique is used to collect the data, and they were analyzed using different approaches to accomplish a number of different goals. 


\section{Objectives and hypothesis}

The central purpose of this thesis is to investigate the behavioral and cerebral changes associated with working memory training and their temporal stability. To this end, behavioral, functional, and anatomical data will be explored before a brief working memory training, immediately after it, and after five weeks with no training. The specific aims of the research are:

1. To investigate, in the short and long-term, the behavioral and fMRI task-activation changes related to $n$-back training effects on the trained task ( $n$-back).

2. To investigate, in the short and long-term, the behavioral and fMRI task-activation changes related to $n$-back training effects on the transfer task (PASAT).

3. To explore, in the short and long-term, the gray matter changes associated with the $n$ back training and their relationship with the behavioral changes.

The following hypotheses were postulated in relation to the aforementioned objectives:

$N$-back and PASAT performance would activate the same brain areas related to working memory: frontal (SFG, ACC, SMA, IFG, AI and DLPFC) and parietal (SPC and IPC), but also temporal and midbrain areas (cerebellum, thalamus and basal ganglia).

* For the trained task, intensive adaptive training on the $n$-back task may lead to behavioral automation of task performance. Participants who trained on the adaptive $n$-back would produce faster responses and respond more accurately than non-trained participants immediately after $n$-back training, and this advantage would be maintained five weeks later.

* It is difficult to have a clear hypothesis about behavioral effects on the transfer task, given all the controversial results in the literature. We believe that due to the 
anticipated similar activation pattern of $n$-back and PASAT, participants who trained on the adaptive $n$-back would respond more accurately than non-trained participants immediately after $n$-back training on the PASAT task, and this advantage would be maintained five weeks later.

* On the trained task, intensive $n$-back training processes would change the functional mechanism of the brain. We expected to find a decreased activation of brain areas already involved in working memory, the frontal and parietal areas, in the short term during the performance of the $n$-back task.

* At the same time, on the transfer task, the same decreased activation in brain areas related to working memory after the $n$-back training processes is expected in the short term, while performing the PASAT task, and again, in frontal and parietal areas.

* After five weeks without training, the cerebral activation during the trained task and during the transfer task in the task-related brain areas would increase to compensate for the lack of training.

* An anatomic change in the brain, specifically in the frontoparietal areas, is expected after the intensive adaptive training on the $n$-back task. That is, the structure of these related areas would show, after training, a change in their gray matter volume. As a result of the continuous use of frontal and parietal areas during training, maintained gray matter changes would be present in these cortical structures.

These hypotheses were tested in an experimental study using the fMRI technique. Their results have been compiled in three original research articles. One has already been published. The other two have been sent for review for publishing in international journals, but they have not been published yet. Therefore, these research articles are included here as they have been sent to or published by the journals. 


\section{Experimental Studies}

Study 1: Long-term brain effects of $N$-back training: an fMRI study

Study 2: Locating neural transfer effects of $N$-back training in the central executive: a longitudinal fMRI study

Study 3: Sustained and transient gray matter volume changes after $N$-back training: a VBM study 


\section{Study 1}

Long-term brain effects of N-back training: an fMRI study

Anna Miró-Padilla, Elisenda Bueichekú, Noelia Ventura-Campos, MariaJesús Flores-Compañ, Maria-Antònia Parcet and César Ávila

Brain Imaging and Behavior

DOI: $10.1007 / \mathrm{s} 11682-018-9925-\mathrm{x}$ 


\begin{abstract}
Neurobehavioral effects of cognitive training have become a popular research issue. Specifically, behavioral studies have demonstrated the long-term efficacy of cognitive training of working memory functions, but the neural basis for this training have been studied only at short-term. Using fMRI, we investigate the cerebral changes produced by brief single $n$-back training immediately and 5 weeks after finishing the training. We used the data from a sample of fifty-two participants who were assigned to either an experimental condition (training group) or a no-contact control condition. Both groups completed three fMRI sessions with the same $n$-back task. Behavioral and brain effects were studied, comparing the conditions and sessions in both groups. Our results showed that $n$-back training improved performance in terms of accuracy and response speed in the trained group compared to the control group. These behavioral changes in trained participants were associated with decreased activation in various brain areas related to working memory, specifically the frontal superior/middle cortex, inferior parietal cortex, anterior cingulate cortex, and middle temporal cortex. Five weeks after training, the behavioral and brain changes remained stable. We conclude that cognitive training was associated with an improvement in behavioral performance and decreased brain activation, suggesting better neural efficiency that persists over time.
\end{abstract}

\title{
KEYWORDS
}

fMRI; $n$-back; cognitive training, long-term effects; working memory 


\section{Introduction}

Working memory is necessary for a significant range of cognitive processes. It is important for everyday life because it is a determinant process in reasoning and in guiding decision-making and behavior (Diamond, 2013). In the past, working memory was defined as a rigid attribute, but it is now known that working memory can be improved when adequate training programs are used (Klingberg, 2010; Morrison \& Chein, 2011; von Bastian \& Oberauer, 2014). These behavioral studies have demonstrated both the immediate effects of this training and its long-term (2-12 months) efficacy once the training has ended. Cerebral changes produced by cognitive training have been studied, but only in the short-term, and so the stability over time of neural changes produced by this training remains unknown. Thus, the overall goal of the present study was to investigate the behavioral and cerebral changes produced by working memory training in the short and long-term.

The simple way of responding to stimuli and the easy management of difficulty makes the $n$-back task an appropriate tool to monitor working memory processes. A large number of studies have been conducted on cognitive training using $n$-back as the main task. All of them suggest that adequate $n$-back training improves task performance in terms of accuracy and reaction times, even with relatively short-term training (e.g. Buschkuehl et al., 2014; Hempel et al., 2004; Jaeggi, Buschkuehl, Jonides, \& Perrig, 2008; Küper \& Karbach, 2016; Li et al., 2008; Salminen, Strobach, \& Schubert, 2012; Schneiders, Opitz, Krick, \& Mecklinger, 2011; Takeuchi et al., 2010; Thompson, Waskom, \& Gabrieli, 2016; Yamashita, Kawato, \& Imamizu, 2015). Generally, participants double or triple their pre-training performance levels (Kundu, Sutterer, Emrich, \& Postle, 2013; Redick et al., 2013; Thompson et al., 2013; Jaeggi et al., 2008). 
The researchers used training programs ranging from 60 to 1500 minutes, with no major differences in improvement (Anguera et al., 2012; Jaeggi et al., 2010b; Schneiders et al., 2012; Vartanian et al., 2013), but little research has focused on the effects of brief $n$-back training. The majority utilized an $n$-back adaptive task during training to manipulate the level of difficulty depending on the participant's performance. Both the single $n$-back and the dual $n$-back task have been used in training studies, with the latter being the most widely utilized, although both have shown efficacy in improving working memory capacity (Jaeggi et al., 2010b). In a very recent study, Küper \& Karbach (2016) compared brief single $n$-back and dual $n$-back training, concluding that both showed equivalent improvement. Moreover, the authors concluded that in short periods of training, single $n$ back training can be more effective than dual $n$-back training (Küper $\&$ Karbach, 2016). Despite all the existing literature, only a few studies have tested the long-term (2-8 months) effects of $n$-back training, and they found that the behavioral changes observed remained stable (Jaeggi, Buschkuehl, Jonides, \& Shah, 2011; Jaeggi, Buschkuehl, Shah, \& Jonides, 2014; Katz et al., 2017; Li et al., 2008), although a decrease was observed in the performance between post-training and the follow-up session (Thompson et al., 2013).

$\mathrm{N}$-back is one of the most common experimental paradigms for functional magnetic resonance imaging (fMRI) studies of working memory (Dobbs \& Rule, 1989; Jaeggi et al., 2010a; Owen, McMillan, Laird, \& Bullmore, 2005; Redick \& Lindsey, 2013; Wager \& Smith, 2003). In a meta-analysis by Owen et al. (2005), twenty-four fMRI studies with healthy subjects who performed the $n$-back task were analyzed in order to find the cerebral regions involved. They studied the brain areas activated depending on the type of stimulus used in the task. Their results showed six cortical regions and two subcortical 
regions activated by verbal stimuli: the lateral premotor cortex, dorsal anterior cingulate and supplementary motor area, dorsolateral and ventrolateral prefrontal cortex, frontal pole, and bilateral and medial posterior parietal cortex; subcortically, the medial and lateral cerebellum and thalamus were activated (Owen et al., 2005).

There are few studies examining the cerebral changes produced by cognitive training on working memory (see the review by Buschkuehl, Jaeggy and Jonides, 2012). In that review, they concluded that there was evidence for brain changes in specific areas in terms of activation, but there was no agreement about whether this activation increased, decreased, or underwent redistribution, or even whether a reorganization of networks took place (Buschkuehl et al., 2012). First, regarding studies that reported increases in brain activation after working memory training, Buschkuehl et al. found limited evidence for this effect. For example, Westerberg \& Klingberg (2007) evaluated the cerebral changes in only three young volunteers after five weeks of working memory training, and they found a significant increased activation in the middle or inferior frontal gyrus and parietal cortex. This finding coincides with the results from a similar prior experiment carried out by this group (Olesen, Westerberg, \& Klingberg, 2004), where they found increased brain activity in the middle frontal gyrus and superior and inferior parietal cortices after five weeks of cognitive training. Second, regarding research that observed both increased and decreased activation (activation redistribution) after working memory training, one study by Dahlin et al. (2008; Experiment 1) stands out. In that study, analyses of pre- and posttraining changes in the fMRI data showed increased activation in the left striatum, temporal, and occipital regions, but also decreased activity in frontal and parietal areas. Participants engaged in five weeks of computer-based updating training on a working 
memory task. Finally, Buschkuehl et al. stated that no noteworthy working-memory training studies showed network reorganization.

Among fMRI studies that reported a decrease in cerebral activation, the majority used the n-back task for training (Hempel et al., 2004; Schneiders et al., 2011, 2012; Schweizer, Grahn, Hampshire, Mobbs, \& Dalgleish, 2013; Thompson et al., 2016). Hempel et al. (2004) carried out four weeks of $n$-back training, and cerebral activation was examined before, after two weeks, and at the end of the training using fMRI. There was no control group. Their results showed an increased activation after two weeks of training in the right inferior frontal gyrus (BA 45) and the right intraparietal sulcus (BA 39/40), but activation decreased in the same areas after four weeks of $n$-back training, forming an inverse U-shaped activation pattern. In addition, Schneiders et al. (2011) used an 8-10 day adaptive $n$-back training (between 400-500 minutes) and performed fMRI pretest and posttest sessions. There were two training groups (visual or auditory $n$-back) and a nocontact control group. The authors observed decreased activation in the right superior middle frontal gyrus (BA 6/9/46) and right posterior parietal lobule (BA 40). In another study, Schneiders et al. (2012) reported the same activation pattern after training their participants for approximately the same length of time as in their prior study, but only on an adaptive auditory $n$-back task. Another study that used $n$-back for training was Schweizer et al. (2013), but in this case, the researchers trained their subjects on an adaptive affective $n$-back task for between 20-30 minutes during 20 days, and they had an active control group for comparison. They found activation decreases in the 3-back load level in the left dorsolateral prefrontal cortex, right superior frontal gyrus, bilateral supramarginal gyrus, bilateral middle temporal gyrus, and bilateral middle occipital lobe. In a recent fMRI study (Thompson et al. 2016) the participants were trained on an 
adaptive dual $n$-back task in 20 sessions distributed across 4 weeks. Before and after the training, volunteers were scanned on the non-adaptive trained task. The authors used both an active and a no-contact control group. Once more, they reported reductions in activation in the bilateral inferior and middle frontal gyrus, insular cortex, and intraparietal sulcus. One study carried out by Vartanian et al. (2013) showed that very brief working memory training also causes cerebral modifications. They performed only 60 minutes of single $n$-back training and reported decreased brain activation in prefrontal areas (BA 46 and 47) (Vartanian et al., 2013). All the studies agree on the brain regions where the activation reductions were found.

On the other hand, Buschkuehl et al. (2014) wanted to test whether brief $n$-back training (less than 3h) increased task-related activation while participants performed difficult levels of the n-back task (4-back) using arterial spin labeling (ASL). They found that activation increased in prefrontal (BA6) and occipital (BA19) areas after training. 4-back places a high demand on cerebral resources, and it would involve an increment in the magnitude of perfusion (Buschkuehl et al., 2014). This activation pattern agrees with the compensation-related utilization of neural circuits hypothesis (CRUNCH) (ReuterLorenz \& Cappell, 2008). This hypothesis postulates that people will activate more cortical regions as task load or resource demands increase. Previous results showed that at lower levels of task demands, older adults activate their task-related brain areas more than younger adults to achieve similar performance as younger adults. However, at harder levels, older people showed reduced task efficiency and less activation than young adults (Heinzel et al., 2016; Heinzel et al., 2014). Thus, in accordance with this hypothesis, the activations in task-related areas would increase or decrease depending on the difficulty of the task. Based on all of this literature, it is difficult to make predictions about cerebral 
changes related to working memory training. Decreased activation is the most frequent result found after cognitive training in studies that use $n$-back, but it has been observed that with high-level demands, the activation increases (Buschkuehl et al., 2014).

In the present study, our main goal was to examine the long-term cerebral changes after working memory training because, to date, we did not find any study that held a followup session to evaluate the stability of the cerebral changes over time. By means of a longitudinal fMRI study, we examined the behavioral and functional data before a brief $n$-back training, immediately after it, and five weeks after finishing the training. Our participants trained for a total of 200 minutes on an adaptive version of single $n$-back on 1-back, 2-back and 3-back levels. That specific training was chosen because, based on previous findings mentioned above, in short periods of training, single $n$-back training would be more effective than dual $n$-back training. In the light of this, we hypothesized that: 1) Training processes would result in decreased activation of brain areas already involved in working memory, the frontal and parietal areas, in the short term; 2) The participants who trained on the adaptive $n$-back would produce faster responses and respond more accurately than non-trained participants immediately after $n$-back training, and this advantage would be maintained five weeks later; 3) After five weeks without training, the cerebral activation in the task-related brain areas would increase to compensate for the lack of training.

\section{Materials and methods}

\subsection{Participants}

Fifty-two healthy right-handed participants (21 male) with ages ranging between 21-26 years (mean age $=22.60 \pm 1.45$ ) participated in this study. Subjects were recruited from 
the student population of the University Jaume I, and none of them reported a previous psychiatric or neurologic diagnosis. Informed consent was obtained from each subject before participation, and they received monetary compensation for their time and effort. Participants were randomly assigned to either an experimental condition (training group) $(\mathrm{N}=25$, mean age $=22.77 \pm 1.5,9$ men $)$ or a control condition (control group) $(\mathrm{N}=27$, mean age $=22.44 \pm 1.4,12$ men). Their intellectual level was assessed with the Matrix Reasoning Test (WAIS-III-R) (trained group: mean $=21.04 \pm 3.42$; control group: mean = $21.81 \pm 2.02)$. The two groups differed only in the training (control group did nothing). The Ethical Committee of the Universitat Jaume I approved the research project.

\subsection{Experimental paradigm}

Both groups completed three fMRI sessions with the same adapted block-design $n$-back task (Zou et al., 2013). A schematic description of the experimental design is represented in Figure 1. The pre-training session, post-training session, and follow-up session correspond to Session 1 (S1), Session 2 (S2), and Session 3 (S3), respectively. Visual stimuli (letters) were presented electronically using E-Prime software (Psychology Software Tools, Pittsburgh, PA), professional version 2.0, installed in a Hewlett-Packard portable workstation (screen-resolution 800 x 600, refresh rate of $60 \mathrm{~Hz}$ ). Participants watched the laptop screen through MRI-compatible goggles (VisuaStim, Resonance Technology, Inc., Northridge, CA, USA), and their responses were collected via MRIcompatible response-grips (NordicNeuroLab, Bergen, Norway). The E-Prime's logfile saved each participant's accuracy and reaction time (RTs) to each stimulus. 
Fig. 1 Schematic description of the experimental design. Both groups performed the same $n$-back task in the three fMRI sessions. Training group data corresponds to the green lines and control group data to the blue lines. Training consisted in an adaptive $n$-back task, 4 sessions, 200 minutes

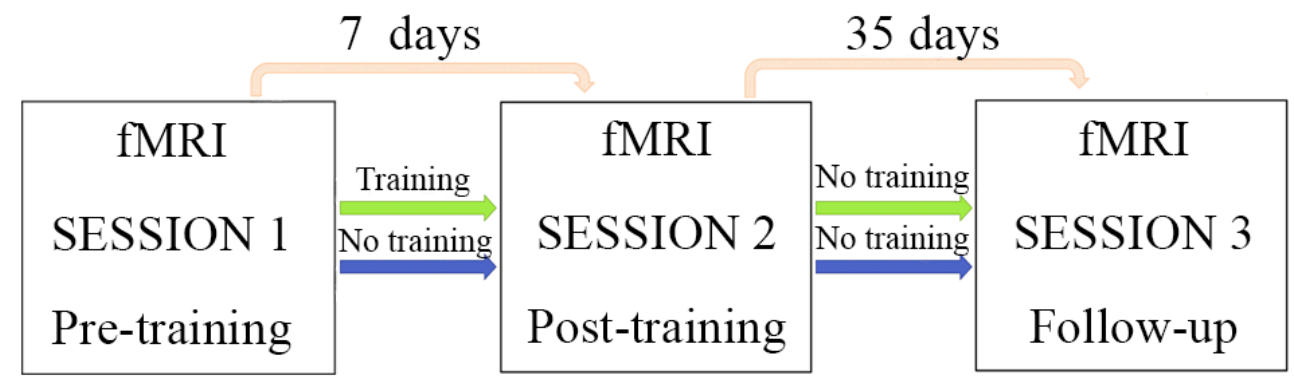

\subsubsection{N-back fMRI task}

The task was presented in three load levels: two working memory blocks (2-back and 3back) and a baseline control task (0-back). In 0-back, subjects pressed the "yes" button when the target (letter X) appeared on the screen, and they responded "no" to any other letters. In the 2-back and 3-back load levels, participants pressed the "yes" button when the current letter shown on the screen matched the one presented 2 or 3 items back, and they pressed "no" when there were no targets (see Figure 2a). Subjects had to give manual responses with only their right hand, responding to targets with their thumb and to non-targets with their forefinger.

With a total of nine blocks, three for each load level, the entire task lasted 11 minutes. Each block lasted 60.7 seconds and consisted of 200 ms of a blank screen, followed by 30 (6 target) consecutive trials of single letter stimuli (500 ms duration, $1500 \mathrm{~ms}$ interstimulus interval) with $500 \mathrm{~ms}$ of a blank screen at the end of each block. In addition, $8000 \mathrm{~ms}$ of a fixation cross and $2000 \mathrm{~ms}$ of an instruction display indicating task difficulty (0-back, 2-back or 3-back) were included before each block (see Figure 2B). There were 270 stimuli in all, and 54 of them were targets. The sequence of the stimuli was pseudorandomized. The visual material comprised 15 different capital letters from the alphabet 
(B, C, D, F, G, H, J, L, N, P, Q, R, S, T and V). Any letter could be a target in 2- and 3back, but in 0-back only the "X" letter was the target. The letters, instructions, and fixation point were presented in the middle of the screen on a white background. All of them were in black ink with a 54-point Arial font. The task did not contain any lures.

Subjects received oral instructions about how to do the task, and they performed a 5minute practicing task. In that, participants performed three blocks, one per load level, with only 15 trials ( 3 targets), in order to become familiar with the stimuli presentation and with the response buttons. A similar laptop with the same display features and the same hardware for manual responses was used outside of the scanner. Participants were asked to answer accurately and as quickly as possible.

Fig. 2 (a) Schematic example of the three load levels of our $n$-back fMRI task. Bold font indicates the correct response. (b) Block timing details of the $n$-back fMRI task

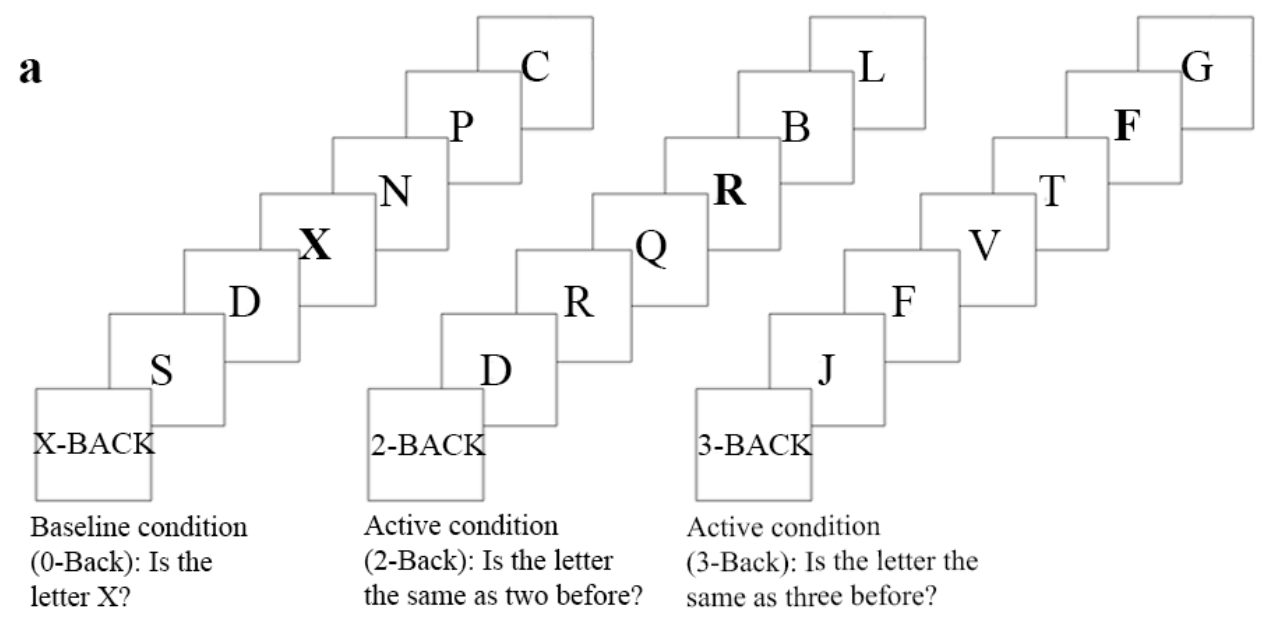

b

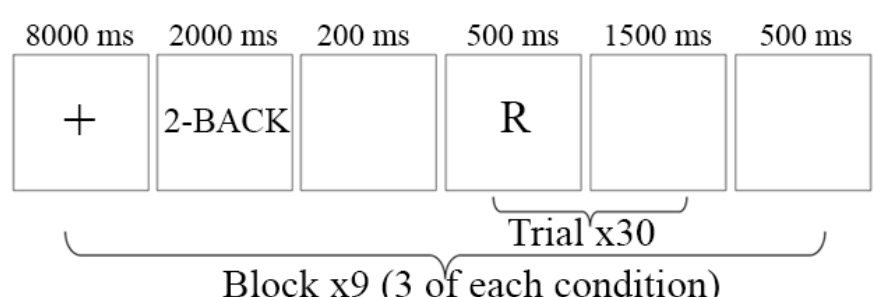




\subsection{2. $N$-back training task}

The training group carried out four consecutive sessions of single $n$-back training after fMRI S1 in our laboratory located at the University. One training session lasted 60 minutes and was distributed in two phases: the learning part and the test part. In the learning part, participants performed an adaptive $n$-back paradigm adapted from Jaeggi et al. (2008) for 50 minutes, whereas in the test part, they performed a simple $n$-back task, which lasted 10 minutes. Therefore, the total training time was approximately 200 minutes, plus 40 minutes for the test part. We used the same laptop as in the fMRI sessions, with the same display features and the same hardware for manual responses. Participants performed only one training session per day. As with our fMRI $n$-back task, no lures were present in our training task.

For the adaptive $n$-back task, we used the same stimuli and block timing as in the $n$-back fMRI task. However, we made some changes: the 0-back load level (0-back) disappeared, a new load level (1-back) was introduced, and participants were given feedback about their performance after each stimulus and at the end of each block. In 1-back, participants pressed the "yes" button when the current letter shown on the screen matched the one presented immediately before, and they pressed "no" in response to any other letters. We lengthened the task to approximately 16 minutes, and subjects performed three runs per training session. Once again, participants were asked to answer accurately and as quickly as possible.

In this task, we changed the level of difficulty by changing the level of " $n$ " $(1,2$ or 3$)$ in order to motivate participants to improve. After each block, the participant's individual performance was analyzed, and the $n$-back level was automatically adjusted. Thus, if the 
participant had at least $90 \%$ correct answers, the level of " $n$ " in the next block was increased by one, but it was decreased by one if accuracy was below $80 \%$. In all other cases, the n-level remained constant (Salminen et al., 2012). In the last run, we increased the percentage by five percent to make it more difficult. Therefore, if the participant had at least $95 \%$ correct answers, the level of " $n$ " was increased by one, whereas it was decreased by one if accuracy was below $85 \%$. Each run started with the minimum level of " $n$ " (1) for motivational reasons (Schneiders et al., 2012). Feedback was introduced after each response: a colored circle appeared for few seconds at the corner of the screen: green if the answer was correct, red if it was an error, and blue if participants did not press any button. Furthermore, at the end of each block, subjects received information about their performance: correct response percentage and reaction time average.

In the test part, participants performed an eight-block $n$-back task. We used the same stimuli and block timing as on the $n$-back fMRI task, but without the 0-back load level. Subjects had no feedback this time. Their results on this test were useful to evaluate their progress on $n$-back.

\subsection{Neuroimaging data acquisition}

Functional MRI data were collected on a 1.5T Siemens Symphony scanner (Erlangen, Germany). The same sequences were used in the three sessions. Participants were placed in a supine position in the MRI scanner, and their heads were immobilized with cushions to reduce motion artifacts. For task-fMRI, a gradient-echo T2*-weighted echo-planar MR sequence covering the entire brain was used $(\mathrm{TR} / \mathrm{TE}=2500 / 49 \mathrm{~ms}$, matrix $=64 \times 64 \times 28$, flip angle $=90^{\circ}$, voxel size $=3.5 \times 3.5 \times 4.48$; slice thickness $=4 \mathrm{~mm}$; slice gap $\left.=0.48 \mathrm{~mm}\right)$. A total of 270 volumes were recorded. The slices were made parallel to the anteriorposterior commissure plane covering the entire brain. Before the functional magnetic 
resonance sequences, a high-resolution structural T1-weighted MPRAGE sequence was acquired $\left(\mathrm{TR}=2200 \mathrm{~ms} ; \mathrm{TE}=3 \mathrm{~ms}\right.$; flip angle $90^{\circ}$, matrix $=256 \times 256 \times 160$; voxel size $=1 \times 1 \times 1 \mathrm{~mm})$.

\subsection{Behavioral analysis}

IBM SPSS Statistics software (Version 22 Armonk, New York, USA) was used to process the behavioral data (accuracy and RTs for participants' performance). A repeatedmeasures $2 \times 3 \times 3$ mixed model ANOVA was conducted for each variable, using Group (training x control) as the between-subjects factor and Load Level (0-back vs. 2-back vs. 3-back) and Session (1 vs. 2 vs. 3) as within-subjects factors. Also, post-hoc analysis was conducted for each variable. With the test part data of the training, a repeated measures 2x4 ANOVA was conducted, with Load Level (2-back vs. 3-back) and Training Session (1 vs. 2 vs. 3 vs. 4 ) as within-subjects factors. Test-retest reliability analyses (re-test correlations) for behavioral control group data are provided in Supplementary

\section{Information.}

\subsection{Neuroimaging analysis}

\subsubsection{Preprocessing}

Preprocessing and statistical analysis of fMRI data were conducted with SPM12 (Wellcome Trust Centre for Neuroimaging, London, UK). We aligned each subject's fMRI data to the AC-PC plane by using his/her anatomical image. The fMRI preprocessing included head motion correction, where the functional images were realigned and resliced to fit the mean functional image. No participant had a head motion of more than $2.5 \mathrm{~mm}$ maximum displacement in any direction or $2.5^{\circ}$ of any angular motion throughout the scan. Afterwards, the anatomical image (T1-weighted) was coregistered to the mean functional image, and the transformed anatomical image was then 
re-segmented. The functional images were spatially normalized to the MNI (Montreal Neurological Institute, Montreal, Canada) space with $3 \mathrm{~mm}^{3}$ resolution, and spatially smoothed with an isotropic Gaussian kernel of $8 \mathrm{~mm}$ FWHM (Full-Width at Half-Maximum).

\subsubsection{First level of analysis}

Statistical analyses were performed in the context of the General Linear Model (Friston et al., 1995) for each participant and for each time point, using SPM12. In the first level analysis, we modeled the load levels of interest corresponding to 2-back > 0-back, 3-back $>0$-back, and 2 and 3-back >0-back. The BOLD signal was estimated by convolving the stimuli onset with the canonical hemodynamic response function. Six motion realignment parameters were included to explain signal variations due to head motion, that is, as covariates of no interest. A high-pass filter (128s) was applied to the functional data to eliminate low-frequency components. Then, contrast images were obtained to directly compare our load levels of interest. For the cross-sectional analysis, the first session (S1) load levels of interest were compared in order to assess differences between the $n$-back load levels before learning.

\subsubsection{Statistical analysis}

In the cross-sectional analysis, a whole-brain one-sample $t$ test was conducted in order to study the brain regions involved in the $n$-back task (2-back and 3-back load levels $>0$ back load level) using the fMRI data collected in S1. In addition, first session data were used to perform a two-sample $t$ test to examine the equality of the brain responses in the two groups, so that between-groups brain differences found in subsequent sessions would be due to training effects. Test-retest reliability analyses (one sample $t$ test) for imaging 
control group data are provided in Supplementary Information.

In the second-level analysis, the longitudinal analysis was performed separately for 2back and 3-back, with interaction analysis between sessions to evaluate: 1) the immediate

effect of training, comparing S2 to S1;2) the long-term effects of training, comparing S3 to S1; and 3) the effects of differences between immediate and long-term effects, comparing S3 to S2. To avoid false positives in the fMRI analyses (Woo, Krishnan, \& Wager, 2014), the statistical criterion was set at $\mathrm{p}<0.05$, and family-wise error (FWE) was cluster-corrected for multiple comparisons (voxel-level uncorrected threshold of $\mathrm{p}<0.001$; specific cluster sizes appear in each result).

\section{Results}

\subsection{Behavioral Results}

\subsubsection{Behavioral fMRI results}

The repeated measures $2 \times 3 \times 3$ mixed-model ANOVA conducted for accuracy yielded main effects for Session $\left(F_{(2.50)}=34.66 \mathrm{p}<.001\right)$ and Load Level $\left(F_{(250)}=42.85 \mathrm{p}<.001\right)$, which means that all the participants reduced their mistakes in the post-training and follow-up sessions, compared to S1, and that the highest accuracy scores were observed during the 0-back. These main effects were driven by significant Group x Session $\left(F_{2500}=7.77 \mathrm{p}=.001\right)$, Load Level x Session $\left(F_{(4,48)}=13.07 \mathrm{p}<.001\right)$ and Load Level x Group $\left(F_{0.50}=7.23 \mathrm{p}=.002\right)$ interactions. The first interaction indicated that trained participants were better than controls during the post-training and follow-up sessions, the second indicated that differences between load levels were greater at pre-training, whereas the third reflected that the training group showed better performance than the control group on 2-back and 3-back. As expected, the Load Level x Session x Group interaction reached significance $\left(F_{(4+4)}=4.01 \mathrm{p}=.007\right)$, which means that the trained group became more 
accurate in the post-training and follow-up sessions than the control group, when performing the 2-back and 3-back load levels (see Figure 3a). Post-hoc analyses revealed that these differences were significant for 3-back vs 0 -back $(\mathrm{p}=.002)$, and they only approached significance for 2-back vs 0-back $(\mathrm{p}=.13)$.

Analyses of RTs scores revealed a similar pattern to that of accuracy. The $2 \times 3 \times 3$ ANOVA also yielded significant main effects for Session $\left(\mathrm{F}_{(250)}=51.59 \mathrm{p}<.001\right)$ and Load Level $\left(\mathrm{F}_{(2.50)}=75.37 \mathrm{p}<.001\right)$. Both groups responded faster in the post-training and follow-up sessions than in the pre-training session. Also, both responded faster in the 0-back load level compared to the 2-back load level, as well as in the 2-back load level compared to the 3-back load level. Significant two-way interactions were obtained for the Group x Session $\left(\mathrm{F}_{(2.50)}=28.14 \mathrm{p}<.001\right)$ and Load Level x Session $\left(\mathrm{F}_{(4,48)}=28.23 \mathrm{p}<.001\right)$ interactions. The first two interactions may be interpreted similarly to accuracy, participants were faster than controls during the post-training and follow-up and the differences between load levels were greater at pre-training. Importantly, all these significant effects were qualified by the three-way Load Level x Session x Group interaction, which was highly significant $\left(F_{(4+8)}=11.34 \mathrm{p}<.001\right)$. As expected, this interaction showed that the training group, compared to the controls, was faster after training and in the follow-up session in the 2-back and 3-back load levels (see Figure 3b). Post-hoc analyses revealed that this effect was significant for both 2-back vs 0-back and 3-back vs 0-back load levels $(\mathrm{p}<.001)$. 
Fig. 3 Results of the behavioral analysis. (a) Correct-response percentage and (a) mean reaction times (in milliseconds) per session have been plotted as a function of load level and time. Pre-training session, post-training session and follow-up session correspond to Session 1, Session 2 and Session 3, respectively. Training group data correspond to the green lines (circles) and control group data to the blue lines (squares). RT $=$ Reaction Time. Error bars represent standard error
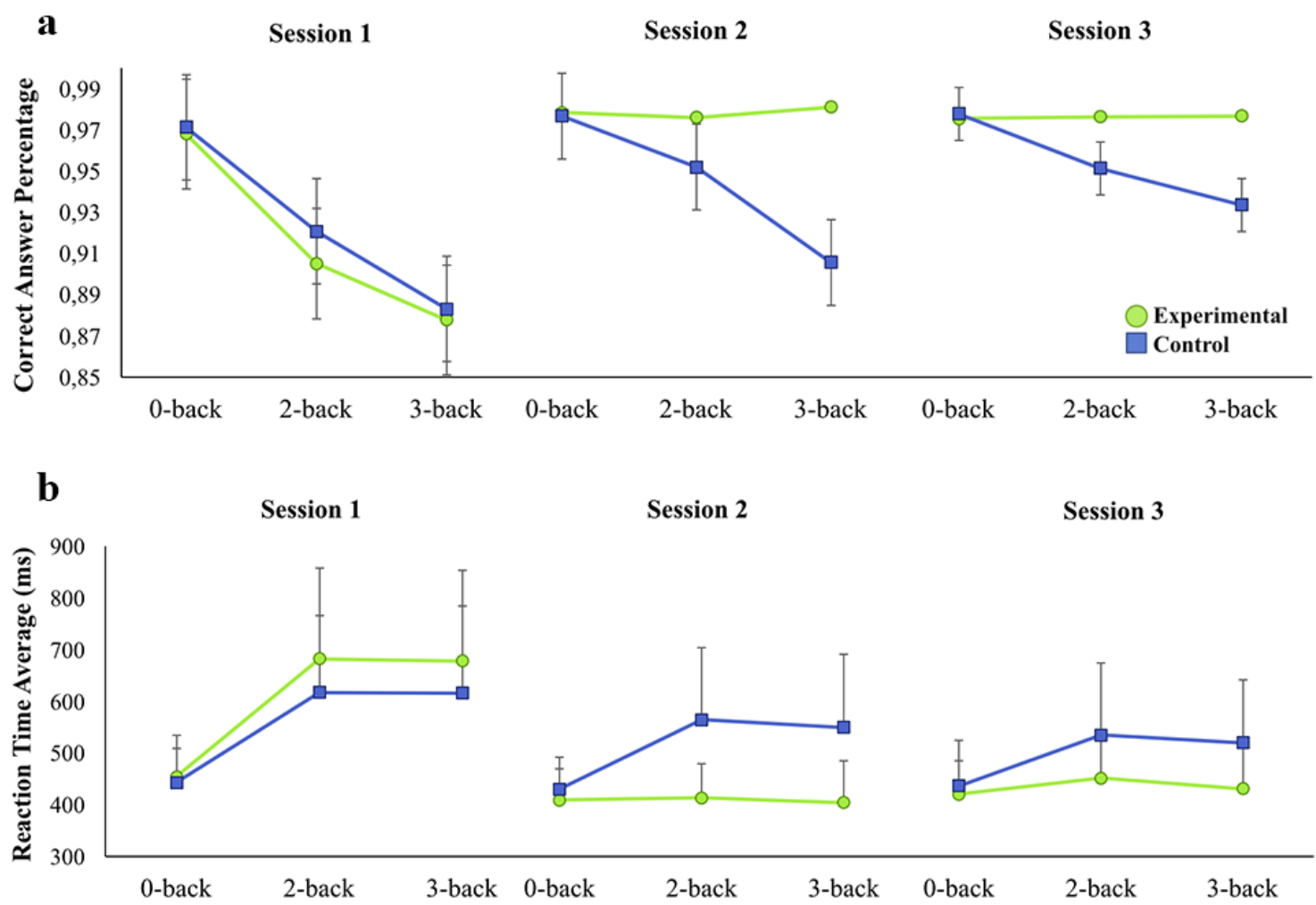

In sum, these results show that there were greater improvements in the 2-back and 3-back load levels after cognitive training, and that these improvements remained stable after five weeks.

\subsubsection{Behavioral training results}

With the behavioral training data for the training group, a repeated-measures $2 \times 4$ ANOVA was conducted with the results of the test part of the training to evaluate their progress on $n$-back. For accuracy training performance, a main effect of Training Session $\left(F_{(32)}=6.49 \mathrm{p}<.05\right)$ and Load Level $\left(F_{(1.29)}=11.99 \mathrm{p}<.05\right)$ was found, indicating participants' improvement, in terms of correct answers from one training session to 
another, and reductions in their mistakes on both 2-back and 3-back. For RT values, we could see a significant effect of Training Session $\left(F_{(323)}=10.35 \mathrm{p}<.001\right)$, which means that subjects' RTs decreased from one training session to another (see Figure 4 for more values). As expected, these results confirmed the great progress of the training group on $n$-back performance after 200 minutes of training.

Fig. 4 Results of the behavioral training analysis of training group. (a) Correct-response percentage and (b) mean reaction times (in milliseconds) per training session have been plotted as a function of load level. 2-back data corresponds to the dark blue bars and 3back data to the light blue bars. TS $=$ training session RT $=$ Reaction Time. Error bars represent standard error
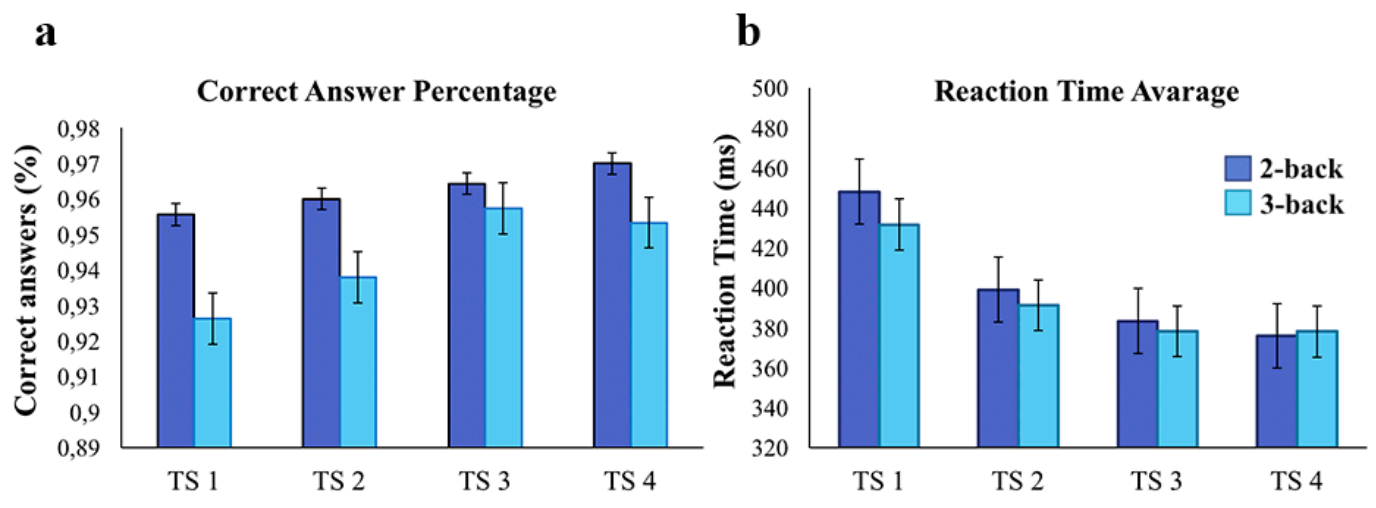

\subsection{Task fMRI Results}

\subsubsection{Cross-Sectional analysis: Task effects at baseline}

A whole-brain one-sample $t$ test was conducted in order to study the brain regions involved in the $n$-back task (2-back and 3-back load levels $>0$-back load level). We used the fMRI data collected in S1. This analysis showed significant cortical and subcortical activations in brain areas related to working memory. Studying the task effects for each 2-back and 3-back load level (2-back $>0$-back and 3-back $>0$-back) separately (see Figure 5), the same areas were activated: bilateral superior, middle and inferior frontal cortex (BA 6/8-11/32/45-48), including supplementary motor area/anterior cingulate gyrus (SMA/ACC) (BA 6/32) and the insula (BA 47), bilateral superior and inferior parietal 
cortex (BA 7/40), including precuneus, and bilateral cerebellum (crus I). Midbrain areas (thalamus and globus pallidus) were not significantly activated in 3-back, whereas they were in 2-back. Results were $\mathrm{p}<.05$ FWE cluster-corrected using a threshold of $\mathrm{p}<.001$ at the uncorrected voxel level with a cluster extension of $k=2504$ voxels for 2-back and $k$ $=143$ for 3-back.

The two-sample $t$ test analysis performed between groups to examine the equality in brain responses in S1 yielded no significant functional differences. As a result, the brain differences found between groups in subsequent sessions were due to training effects. The threshold was $p<0.001$ uncorrected at the voxel level.

Fig. $5 \mathrm{~N}$-back general task activations. Both load levels presented an activation in the same areas, with some exception in cortical structures. (a) 2-back load level, represents the contrast: 2-back $>0$-back and (b) 3-back load level, represents the contrast: 3-back $>0$ back. Results were $p<.05$ FWE-cluster corrected with a cluster criteria of $k=2504$ voxels for 2-back and $k=143$ for 3-back. Left (L) and right (R). Coordinates are in the MNI space. Color bars express $t$-scores

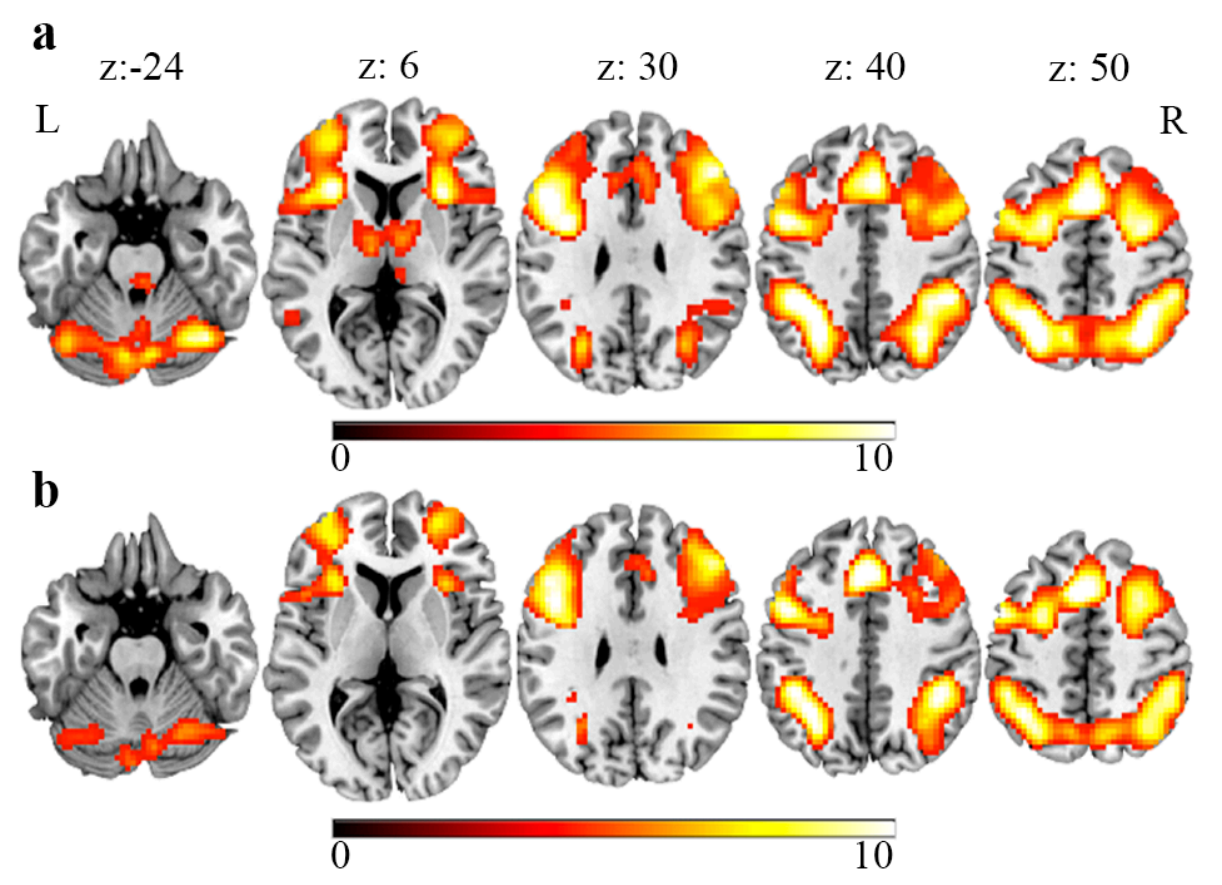




\subsubsection{Learning effects}

To study the effects of training on the brain, an interaction analysis was conducted. Therefore, a 2x2 ANOVA (Group x Session) was carried out separately for each load level (2back and 3back). When studying the training effects by comparing S1 vs S2 and S1 vs S3 in the 2-back load level, we found similar results. These interaction analyses yielded activations in the bilateral superior frontal cortex (BA 8-9), including the SMA/ACC (BA 6/32), dorsolateral prefrontal cortex (BA 9/46), inferior frontal cortex (BA 44-46), and right inferior parietal cortex (IPC) (BA 39-40), in the trained group compared to the control group (see Figure 6 and Table 1). The reverse contrast yielded no significant effects. Results were $\mathrm{p}<.05$ FWE cluster-corrected using a threshold of $\mathrm{p}<.001$ at the uncorrected voxel level and a cluster extension of $\mathrm{k}=125$ voxels and $\mathrm{k}=$ 87 voxels, respectively.

Fig. 6 Results of the adaptive $n$-back post-training effects for 2-back load level: (a) represents the contrast: Trained group $(\mathrm{S} 1>\mathrm{S} 2)>$ Control group $(\mathrm{S} 1>\mathrm{S} 2)$ and (b) represents the contrast: Trained group $(\mathrm{S} 1>\mathrm{S} 3)>$ Control group $(\mathrm{S} 1>\mathrm{S} 3)$. Results were $\mathrm{p}<.05$ FWE cluster-corrected using a threshold of $\mathrm{p}<.001$ at the uncorrected voxel level and a cluster extension of $\mathrm{k}=125$ voxels and $\mathrm{k}=87$ voxels respectively. Left (L) and right (R). Coordinates are in the MNI space. Color bars express $t$-scores

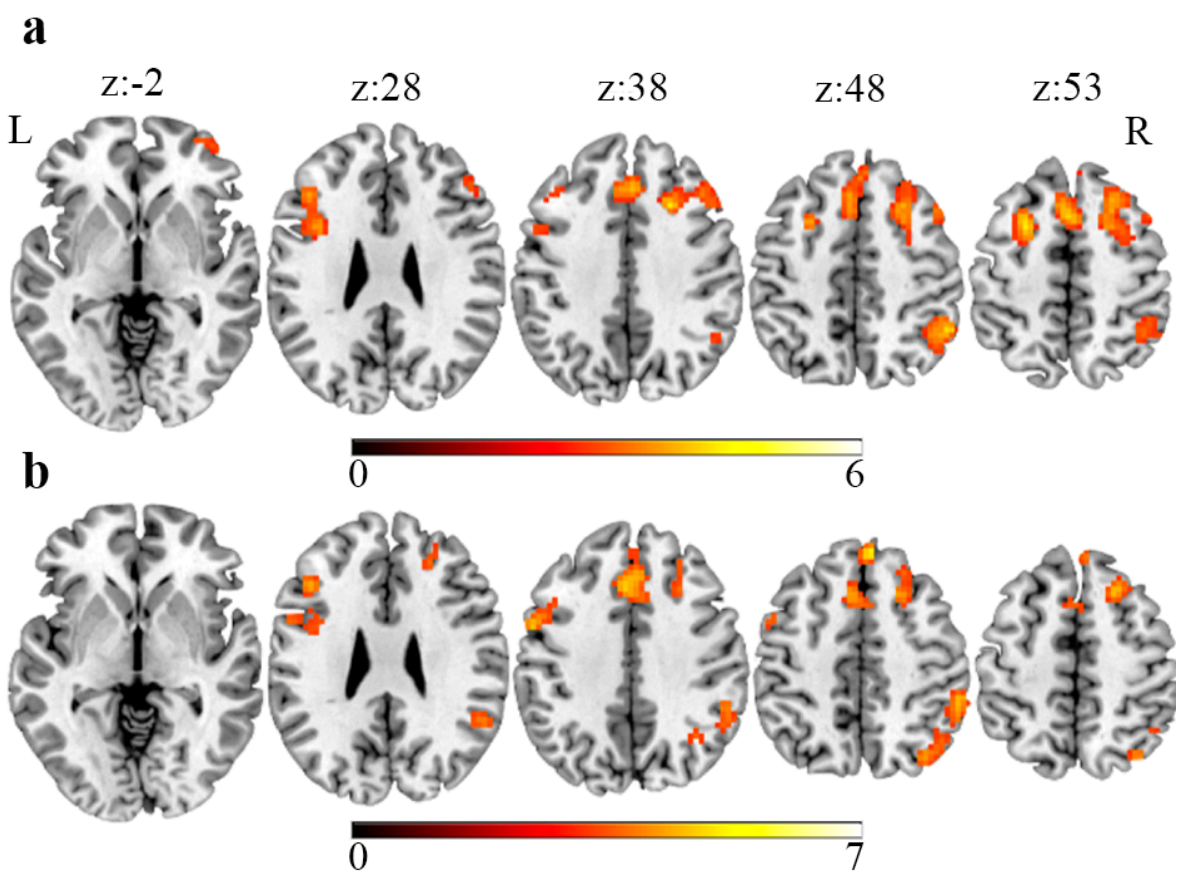


Table 1. List of brain activations as a result of the post-training session and follow-up session in 2-back load level between groups comparison: a) comparing Session 1 with Session 2 and b) comparing Session 1 with Session 3.

\begin{tabular}{|c|c|c|c|c|c|c|}
\hline & \multirow[b]{2}{*}{ BA } & \multicolumn{5}{|c|}{ MNI SPACE } \\
\hline & & Cluster extent & $\mathbf{x}$ & $\mathbf{y}$ & $\mathbf{z}$ & Z-value \\
\hline \multicolumn{7}{|c|}{ a) Trained Group $($ S1>S2) > Control Group $($ S1>S2) } \\
\hline L Frontal Superior & 6 & 132 & -24 & -1 & 59 & 4.67 \\
\hline R Frontal Middle & 46 & 570 & 42 & 53 & 8 & 4.47 \\
\hline R Parietal Inferior & 40 & 130 & 51 & -49 & 47 & 4.21 \\
\hline SMA & 6 & 248 & 0 & 14 & 53 & 4.16 \\
\hline L Frontal Inferior & 48 & 125 & -42 & 26 & 29 & 3.87 \\
\hline \multicolumn{7}{|c|}{ b) Trained Group (S1>S3) > Control Group (S1>S3) } \\
\hline R Frontal Middle & $9 / 8$ & 235 & 3 & 44 & 44 & 4.64 \\
\hline $\mathrm{R}$ Parietal Inferior & 40 & 121 & 54 & -40 & 44 & 4.61 \\
\hline R Frontal Superior & 8 & 141 & 24 & 26 & 59 & 4.53 \\
\hline L Frontal Middle & 6 & 203 & -54 & 2 & 38 & 4.24 \\
\hline $\mathrm{R}$ Parietal Superior & 7 & 87 & 36 & -70 & 50 & 4.04 \\
\hline
\end{tabular}

FOOTNOTES: Results were $\mathrm{p}<0.05$ FWE cluster-corrected using a threshold of $\mathrm{p}<$ 0.001 at the uncorrected voxel level, and a cluster extension of $\mathrm{k}=125$ voxels and $\mathrm{k}=87$ voxels respectively. $\mathrm{L}=$ Left. $\mathrm{R}=$ Right. $\mathrm{BA}=$ Brodmann Area. SMA = supplementary motor area.

Regarding the 3-back load level, there were differences in the affected areas depending on the sessions compared. In the Trained group $(S 1>S 2)>$ Control group $(S 1>S 2)$ contrast, the analyses showed activations in the bilateral superior/middle frontal cortex (BA 8-11/46), including the SMA/ACC (BA 6/32), left insula, bilateral IPC (BA 39-40), and left temporal middle cortex (BA 21), in the trained group compared to the control group. On the other hand, in the Trained group $(S 1>S 3)>$ Control group $(S 1>S 3)$ contrast, the difference was found in the right IPC (BA 40), bilateral insula, SMA/ACC (6/32), bilateral inferior frontal cortex (BA 44-45), and dorsolateral prefrontal cortex (BA 9). The reverse contrasts yielded no significant differences. In Figure 7 and Table 2, we have included the results and values of these comparisons for each load level. The threshold was at $\mathrm{p}<.05 \mathrm{FWE}$ cluster-corrected using an auxiliary threshold of $\mathrm{p}<.001$ at 
the uncorrected voxel level and a cluster extension of $\mathrm{k}=89$ voxels and $\mathrm{k}=67$ voxels, respectively.

Fig. 7 Results of the adaptive $n$-back post-training effects for 3-back load level: (a) represents the contrast: Trained group $(\mathrm{S} 1>\mathrm{S} 2)>$ Control group $(\mathrm{S} 1>\mathrm{S} 2)$ and (b) represents the contrast: Trained group $(\mathrm{S} 1>\mathrm{S} 3)>$ Control group $(\mathrm{S} 1>\mathrm{S} 3)$. Results were $\mathrm{p}<.05 \mathrm{FWE}$ cluster-corrected using a threshold of $\mathrm{p}<.001$ at the uncorrected voxel level and a cluster extension of $k=89$ voxels and $k=67$ voxels respectively. Left (L) and right (R). Coordinates are in the MNI space. Color bars express $t$-scores

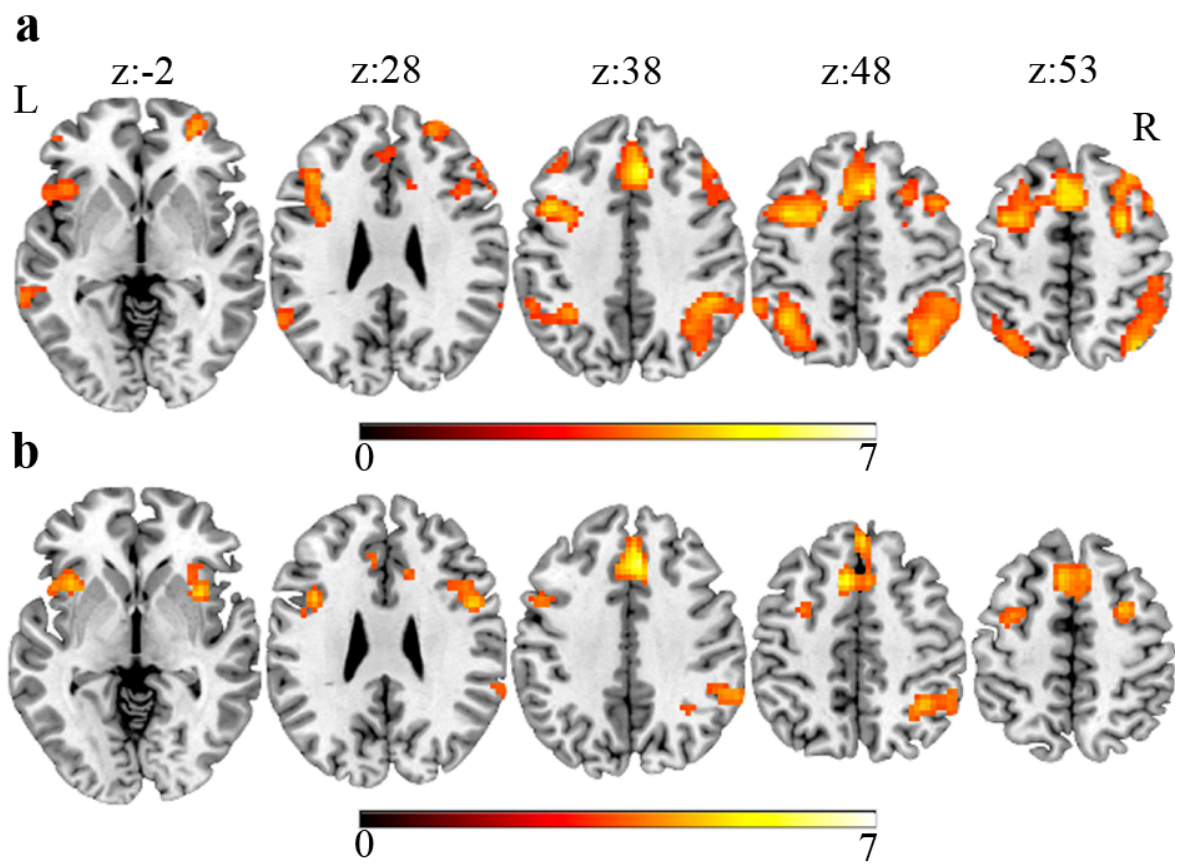

When studying the stability of the effects of the working memory training over time, an interaction analysis was also conducted (Trained group $(S 2>S 3)>$ Control group $(S 2>S 3))$ separately for 2-back and 3-back. No significant effects were found in either load level or any comparison. The threshold was $p<.001$ uncorrected at the voxel level.

In sum, comparing the pre-training session to the post-training session and the follow-up session, decreased activation was found in working memory brain areas when studying the 2-back or 3-back load level. These results were found for trained participants 
compared to controls. However, no differences were found between the post-training and follow-up sessions, which means that the effects of training remained stable after 5 weeks.

Table 2. List of brain activations as a result of the post-training session and follow-up session in 3-back load level between groups comparison: a) comparing Session 1 with Session 2 and b) comparing Session 1 with Session 3.

\begin{tabular}{|c|c|c|c|c|c|c|}
\hline & \multirow[b]{2}{*}{$\mathbf{B A}$} & \multicolumn{5}{|c|}{ MNI SPACE } \\
\hline & & Cluster extent & $\mathbf{x}$ & $\mathbf{y}$ & $\mathbf{z}$ & Z-value \\
\hline \multicolumn{7}{|c|}{ a) Trained Group $($ S1 $>$ S2) $>$ Control Group $($ S1 $>$ S2) } \\
\hline R Frontal Superior & $32 / 6$ & 1380 & 6 & 29 & 41 & 5.48 \\
\hline L Frontal Middle & 10 & 164 & -36 & 53 & 11 & 4.78 \\
\hline R Frontal Middle & 6 & 821 & 30 & 2 & 53 & 4.76 \\
\hline L Parietal Inferior & 40 & 342 & -39 & -55 & 47 & 4.65 \\
\hline R Parietal Inferior & 40 & 579 & 48 & -43 & 44 & 4.65 \\
\hline L Temporal Middle & 21 & 89 & -66 & -43 & 2 & 4.03 \\
\hline \multicolumn{7}{|c|}{ b) Trained Group (S1>S3) > Control Group (S1>S3) } \\
\hline R Frontal Middle & $32 / 9$ & 454 & 6 & 29 & 38 & 5.08 \\
\hline L Insula & $48 / 47$ & 122 & -33 & 17 & -7 & 4.77 \\
\hline R Insula & & 110 & 36 & 11 & -1 & 4.26 \\
\hline R Frontal Inferior & 44 & 138 & 48 & 5 & 29 & 4.23 \\
\hline L Frontal Inferior & 44 & 108 & -39 & 8 & 29 & 4.16 \\
\hline $\mathrm{R}$ parietal Inferior & 40 & 199 & 36 & -52 & 47 & 4.09 \\
\hline L Frontal Middle & 6 & 67 & -33 & 1 & 53 & 3.74 \\
\hline
\end{tabular}

FOOTNOTES: Results were $p<0.05$ FWE cluster-corrected using a threshold of $p<$ 0.001 at the uncorrected voxel level, and a cluster extension of $\mathrm{k}=89$ voxels and $\mathrm{k}=67$ voxels respectively. $\mathrm{L}=$ Left. $\mathrm{R}=$ Right. $\mathrm{BA}=$ Brodmann Area.

\section{Discussion}

The present fMRI research focused on studying the behavioral and neural changes associated with working memory training and their stability over time. To accomplish this, we randomly separated participants into two groups (training or control group), and both groups completed three fMRI sessions performing the same $n$-back task. The training group was trained outside of the scanner on an adaptive version of the single $n$ back task for 200 minutes in four training sessions between the pre-training and posttraining sessions. A follow-up session was held after five weeks of no training. Our results 
showed significant behavioral and functional differences between groups related to the working memory training. $N$-back training improved the performance on the task, and these behavioral changes were accompanied by decreased activation in diverse brain areas related to working memory, specifically, in the frontal superior/middle cortex, inferior parietal cortex, anterior cingulate cortex, and temporal middle cortex. Importantly, five weeks after the training, the behavioral and brain changes remained stable. Our results demonstrate that our cognitive training program improved behavioral performance and cause cerebral modifications that persist over time when compared with a no-contact control group.

Training effects were observed in terms of accuracy and RTs. Generally, all the participants in both groups improved their performance in the post-training session compared to the pre-training session. Control group improvements could be explained by retest effects due to task repetition, as reported in previous cognitive studies (Jaeggi et al., 2008; Schneiders et al., 2011). However, the training group reduced their errors and RTs significantly more than the control group in both working memory load levels. As expected, 200 minutes of working memory training on our adaptive single $n$-back task yielded an improvement in performance in terms of accuracy and reaction times. Our follow-up findings also showed that these behavioral changes remained stable five weeks after completing the training. A non-significant decrease was noted in the performance from the post-training session to the follow-up session because there was no additional training. These results agree with previous $n$-back training studies (e.g. Jaeggi et al., 2011; Thompson et al., 2013, 2016). 
The $n$-back task activation pattern reported here, which includes the frontal, parietal, cerebellar, and subcortical areas, coincides with previous neuroimaging studies (Owen et al., 2005). All the activations found were bilateral and located specifically at the SMA/ACC (BA 6/32), superior, middle, and inferior frontal cortex, including the anterior insula, superior and inferior parietal cortex (BA7/40), including precuneus, cerebellum (crus I), and thalamus. Formerly, in working memory, the prefrontal cortex was considered a warehouse of information (Smith \& Jonides, 1999), but current views give the prefrontal cortex the function of controlling the cognitive processing of information, selecting stimuli, and producing adequate responses (Postle, 2006). There is increasing evidence supporting this view (Lara \& Wallis, 2015). In addition, executive manipulation of acquired facts has been associated with the parietal lobe (Koenigs, Barbey, Postle, \& Grafman, 2009), as well as the storage function of working memory (Owen et al., 2005) as well as attentional processes of working memory (Berryhill, Chein, \& Olson, 2011). Regarding the subcortical areas, the cerebellum assumes cognitive information processing functions due to its connections with the prefrontal cortex (Hayter, Langdon, \& Ramnani, 2007; Vandervert, 2009). The thalamus, due to its attentional role of filtering relevant information, helps the prefrontal cortex in its working memory function (Watanabe \& Funahashi, 2012).

Our findings are generally consistent with previous $n$-back functional neuroimaging studies that report decreased activation after training (Schneiders et al., 2011, 2012; Schweizer et al., 2013; Thompson et al., 2016). In relation to training activation changes, our imaging data revealed that participants who belonged to the training group showed decreased activation in various cerebral areas related to working memory. Decreased activation has been interpreted as an indication of better neural efficiency in these areas, 
thus improving their function. This decline in cerebral activation may allow participants to respond more quickly and make fewer mistakes (Buschkuehl et al., 2014). Kelly, Foxe, \& Garavan (2006) noted that this effect of decreased activation is typically observed after training on higher cognitive tasks, and they stated that lower activation is associated with increased neural efficiency, which means that fewer neurons are needed to give a fast and accurate answer to the task. However, some studies have criticized the better neural efficiency explanation for the decreases in activation for being overly simple and unclear (Constantinidis \& Klingberg, 2016; Poldrack, 2015). In his review, Poldrack (2015) viewed efficiency as inverted energy for the transmission of information in the brain networks. He highlighted the need for new studies and models to examine the neural changes, and he reported that identifying potential activation effects may lead to future mechanistic explanations. Therefore, although a decrease in activation is often interpreted as an increase in neural efficiency in the literature, our data did not demonstrate the underlying cellular mechanism, but instead they pointed to the areas of change after working memory training. The bilateral superior frontal cortex (BA 8-9), IPC (BA 40) and SMA/ACC (BA 6/32) were the areas affected by this activation reduction in both 2back and 3-back. During 3-back performance, we also found a decreased activation in the left insula and left middle temporal cortex (BA 21). The main effect only on 3-back performance in these specific areas may be due to more demanding load levels than those of 2-back (Thompson et al., 2016).

The activation decreases in the superior part of the frontal cortex in both hemispheres were expected because the dorsolateral prefrontal cortex is strongly involved in working memory processes (Lara \& Wallis, 2015). It is essential for continuous updating processes, attention focus, and ordering and selecting stimuli, which are fundamental 
processes in performing the $n$-back task successfully. Other areas related to working memory where this effect was found were the IPC, SMA and ACC. Regarding IPC, this area is in charge of the phonological store, as demonstrated in studies with patients with lesions in this area (Baldo \& Dronkers, 2006). This storage of verbal information is necessary to carry out our $n$-back task because we used letters as stimuli. Moreover, the IPC is typically activated when an attentionally-demanding maintenance strategy is used (Berryhill et al., 2011). With regard to SMA, this area has been related to the planning of sequences of movement, motor learning, and motor activation of the hand. In our case, as participants had to give the answer by pressing a button with their right hand, the activation decreases in these areas were accompanied by a decrease in RTs. On the other hand, the ACC has been related to error detection (Bush, Luu, \& Posner, 2000), which is crucial to carry out our working memory task. Menon \& Uddin (2010) said that the ACC and the insula work together in the detection of important stimuli and in initiating attentional control signals. Regarding the middle temporal cortex, further investigation is needed to determine its exact relationship with working memory.

One of the novel goals of the present study was to investigate the long-term effects of cognitive training. We did not find any longitudinal fMRI research that studies the stability of brain changes produced by working memory training, and so we cannot compare our functional results. Our fMRI analysis showed no significant changes between the two sessions, which means that the changes due to $n$-back training remained stable after the training ended. Our findings showed that the main effect that occurred between the pre-training and post-training sessions (decrease in activation) was present in the same areas when comparing the pre-training session with the follow-up session. Thus, our results demonstrate that the behavioral and cerebral changes produced by 
working memory training remain stable after five weeks without training. The stability of these brain changes after five weeks could suggest an improved efficiency of these areas because we found no modifications in the results when comparing S2 and S3 in the behavioral analysis or the fMRI analysis. The follow-up session seems to be a necessary component of any working memory training paradigm designed to create enduring improvements (Thompson et al., 2013).

Overall results are partially consistent with the CRUNCH theory (Reuter-Lorenz \& Cappell, 2008). In fact, the reduction in activations after training in the training group may be explained by the theory because training reduced the required task demands. However, we also expected that 5 weeks of no training would increase the activations on the task, but this was not the case, indicating that the positive effects of training were maintained without any loss for at least five weeks, as the behavioral and neural data suggest.

This study has a few limitations. We used a no-contact control group that did not receive any training. The training group came to our laboratory on four consecutive days, and they had more contact with the experimenters than the control group did, which may result in motivational differences between the two groups in terms of task efficiency. Nonetheless, the control group improved their performance from S1 to S2 and from S2 to S3. Although this may be attributed to the re-test effect, we also note that this improvement would not have taken place if there had been a lack of motivation. In any case, in future studies, active control groups should be included in the study design because the observed gains may not be due to working memory training per se, but to the training in general. Another limitation may be the short training period (200 minutes), 
although some studies have used the same training time or less and showed behavioral improvements and cerebral changes (Buschkuehl et al., 2014; Jaeggi et al., 2008; Küper \& Karbach, 2016; Vartanian et al., 2013; Yamashita et al., 2015). In addition, we have chosen a brief single $n$-back training with an eye on future clinical interventions. A long training protocol might be difficult and costly for patients and institutions. Therefore, we wanted to evaluate the effects of this kind of short working memory training regime on healthy controls to allow comparisons with clinical populations in future studies. The practice is limited to 1-, 2- and 3-back levels, which may not seem challenging, but 3back is considered a highly demanding task, and participants reported that they always tried to get better results because they could see their correct response percentage and reaction time average. Finally, the expression "long-term" should be used with care because five weeks is not much time compared to other studies investigating long-term effects for at least 3 months to 1 year (e.g. Jaeggi et al., 2014; Katz et al., 2017; Thompson et al., 2013). In any case, we think it is relevant that this is the first manuscript to investigate brain reorganization weeks after the training is over. Future studies should determine this stability in longer retest periods.

In conclusion, $n$-back training not only improves behavioral performance, but it also causes cerebral modifications as signaled by the decrease in the activation of various brain areas related to working memory. These behavioral and neural changes are stable and persist after weeks with no training on the task. The future challenge is to determine whether this kind of training has the same effects in a clinical population and could be translated into beneficial and long-lasting treatments, and test whether these changes last longer than five weeks. 


\section{Compliance with Ethical Standards:}

Funding: This research was supported by grants from Jaume I University (P1•1B201363) and Ministerio de Economia y Competitividad (PSI2013-47504-R and PSI201678805-R). Authors AMP, EB and MJFC were supported by pre-doctoral graduate program grants (Jaume I University FPI to AMP; National FPU to EB; and National FPI to MJFC.

Conflict of Interest: The authors declare that they have no conflict of interest.

Ethical approval: All procedures performed in studies involving human participants were in accordance with the ethical standards of the institutional Review Board of the Universitat Jaume I and with the 1964 Helsinki declaration and its later amendments or comparable ethical standards.

Informed consent: Informed consent was obtained from all individual participants included in the study.

\section{References}

Anguera, J. A., Bernard, J. A., Jaeggi, S. M., Buschkuehl, M., Benson, B. L., Jennett, S., ... Seidler, R. D. (2012). The effects of working memory resource depletion and training on sensorimotor adaptation. Behavioural Brain Research, 228(1), 107-115. http://doi.org/10.1016/j.bbr.2011.11.040

Baldo, J. V, \& Dronkers, N. F. (2006). The role of inferior parietal and inferior frontal cortex in working memory. Neuropsychology, 20(5), 529-538. http://doi.org/10.1037/0894-4105.20.5.529

Berryhill, M. E., Chein, J., \& Olson, I. R. (2011). At the intersection of attention and memory: the mechanistic role of the posterior parietal lobe in working memory. Neuropsychologia, 49(5), 1306-15. http://doi.org/10.1016/j.neuropsychologia.2011.02.033

Buschkuehl, M., Hernandez-Garcia, L., Jaeggi, S. M., Bernard, J. a, \& Jonides, J. (2014). Neural effects of short-term training on working memory. Cognitive, Affective \& Behavioral Neuroscience, 14(1), 147-60. http://doi.org/10.3758/s13415-013-02449

Buschkuehl, M., Jaeggi, S. M., \& Jonides, J. (2012). Neuronal effects following working memory training. Developmental Cognitive Neuroscience, 2, Supplem, S167-S179. http://doi.org/10.1016/j.den.2011.10.001

Bush, G., Luu, P., \& Posner, M. (2000). Cognitive and emotional influences in anterior cingulate cortex. Trends Cogn. Sci., 4(6), 215-222. http://doi.org/10.1016/S13646613(00)01483-2

Constantinidis, C., \& Klingberg, T. (2016). The neuroscience of working memory capacity and training. Nature Reviews Neuroscience, 17(7), 438-449. http://doi.org/10.1038/nrn.2016.43

Dahlin, E., Neely, A. S., Larsson, A., Backman, L., \& Nyberg, L. (2008). Transfer of Learning After Updating Training Mediated by the Striatum. Science, 320(5882), 
1510-1512. http://doi.org/10.1126/science.1155466

Diamond, A. (2013). Executive functions. Annual Review of Sychology, 64, 135-68. http://doi.org/10.1146/annurev-psych-113011-143750

Dobbs, a R., \& Rule, B. G. (1989). Adult age differences in working memory. Psychology and Aging, 4(4), 500-503. http://doi.org/10.1037//0882-7974.4.4.500

Friston, K. J., Holmes, A. P., Worsley, K. J., Poline, J.-P., Frith, C. D., \& Frackowiak, R. S. J. (1995). Statistical Parametric Maps in Functional Imaging: A General Linear Approach. Human Brain Mapping, 2(4), 189-210. http://doi.org/10.1002/hbm.460020402

Hayter, A. L., Langdon, D. W., \& Ramnani, N. (2007). Cerebellar contributions to working memory. NeuroImage, 36(3), 943-954. http://doi.org/10.1016/j.neuroimage.2007.03.011

Heinzel, S., Lorenz, R. C., Brockhaus, W.-R., Wüstenberg, T., Kathmann, N., Heinz, A., \& Rapp, M. A. (2014). Working Memory Load-Dependent Brain Response Predicts Behavioral Training Gains in Older Adults. The Journal of Neuroscience, 34(4), 1224-1233. http://doi.org/10.1523/JNEUROSCI.2463-13.2014

Heinzel, S., Lorenz, R. C., Pelz, P., Heinz, A., Walter, H., Kathmann, N., ... Stelzel, C. (2016). Neural correlates of training and transfer effects in working memory in older adults. NeuroImage, 134, 236-249. Retrieved from http://dx.doi.org/10.1016/j.neuroimage.2016.03.068 \npapers://70c55321-b12646ea-ac63-5b3363fb7a4a/Paper/p2623

Hempel, A., Giesel, F. L., Garcia Caraballo, N. M., Amann, M., Meyer, H., Wüstenberg, T., ... Schröder, J. (2004). Plasticity of Cortical Activation Related to Working Memory during Training. American Journal of Psychiatry, (April), 745-747. http://doi.org/10.1176/appi.ajp.161.4.745

Jaeggi, S. M., Buschkuehl, M., Jonides, J., \& Perrig, W. J. (2008). Improving fluid intelligence with training on working memory. Proceedings of the National Academy of Sciences of the United States of America, 105(19), 6829-6833. http://doi.org/10.1073/pnas.0801268105

Jaeggi, S. M., Buschkuehl, M., Perrig, W. J., \& Meier, B. (2010a). The concurrent validity of the N -back task as a working memory measure. Memory, 18(4), 394-412. http://doi.org/10.1080/09658211003702171

Jaeggi, S. M., Studer-Luethi, B., Buschkuehl, M., Su, Y.-F., Jonides, J., \& Perrig, W. J. (2010b). The relationship between n-back performance and matrix reasoning implications for training and transfer. Intelligence, 38(6), 625-635. http://doi.org/10.1016/j.intell.2010.09.001

Jaeggi, S. M., Buschkuehl, M., Jonides, J., \& Shah, P. (2011). Short- and long-term benefits of cognitive training. Proceedings of the National Academy of Sciences, 108(25), 10081-10086. http://doi.org/10.1073/pnas.1103228108

Jaeggi, S. M., Buschkuehl, M., Shah, P., \& Jonides, J. (2014). The role of individual differences in cognitive training and transfer. Memory \& Cognition, 42, 464-80. http://doi.org/10.3758/s13421-013-0364-z

Katz, B., Au, J., Buschkuehl, M., Abagis, T., Zabel, C., Jaeggi, S. M., \& Jonides, J. (2017). Individual Differences and Long-term Consequences of tDCS-augmented Cognitive Training. Journal of Cognitive Neuroscience, 29(9), 1498-1508. http://doi.org/10.1162/jocn_a_01115

Kelly, C., Foxe, J. J., \& Garavan, H. (2006). Patterns of Normal Human Brain Plasticity After Practice and Their Implications for Neurorehabilitation. Archives of Physical Medicine and Rehabilitation, 87(12 SUPPL.), 20-29. http://doi.org/10.1016/j.apmr.2006.08.333 
Klingberg, T. (2010). Training and plasticity of working memory. Trends in Cognitive Sciences, 14(7), 317-324. http://doi.org/10.1016/j.tics.2010.05.002

Koenigs, M., Barbey, A. K., Postle, B. R., \& Grafman, J. (2009). Superior parietal cortex is critical for the manipulation of information in working memory. The Journal of Neuroscience : The Official Journal of the Society for Neuroscience, 29(47), 149806. http://doi.org/10.1523/JNEUROSCI.3706-09.2009

Kundu, B., Sutterer, D. W., Emrich, S. M., \& Postle, B. R. (2013). Strengthened effective connectivity underlies transfer of working memory training to tests of short-term memory and attention. The Journal of Neuroscience: The Official Journal of the Society for Neuroscience, 33(20), 8705-15. http://doi.org/10.1523/JNEUROSCI.5565-12.2013

Küper, K., \& Karbach, J. (2016). Increased training complexity reduces the effectiveness of brief working memory training: evidence from short-term single and dual n-back training interventions. Journal of Cognitive Psychology, 28(2), 199-208. http://doi.org/10.1080/20445911.2015.1118106

Lara, A. H., \& Wallis, J. D. (2015). The role of prefrontal cortex in working memory: a mini review. Frontiers in Systems Neuroscience, 9(December), 1-7. http://doi.org/10.3389/fnsys.2015.00173

Li, S.-C., Schmiedek, F., Huxhold, O., Röcke, C., Smith, J., \& Lindenberger, U. (2008). Working memory plasticity in old age: Practice gain, transfer, and maintenance. Psychology and Aging, 23(4), 731-742. http://doi.org/10.1037/a0014343

Menon, V., \& Uddin, L. Q. (2010). Saliency, switching, attention and control: a network model of insula function. Brain Structure and Function, 214(5-6), 655-667. http://doi.org/10.1007/s00429-010-0262-0

Morrison, A. B., \& Chein, J. M. (2011). Does working memory training work? The promise and challenges of enhancing cognition by training working memory. Psychonomic Bulletin \& Review, 18(1), 46-60. http://doi.org/10.3758/s13423-0100034-0

Olesen, P. J., Westerberg, H., \& Klingberg, T. (2004). Increased prefrontal and parietal activity after training of working memory. Nature Neuroscience, 7(1), 75-9. http://doi.org/10.1038/nn1165

Owen, A. M., McMillan, K. M., Laird, A. R., \& Bullmore, E. (2005). N-back working memory paradigm: a meta-analysis of normative functional neuroimaging studies. Human Brain Mapping, 25(1), 46-59. http://doi.org/10.1002/hbm.20131

Poldrack, R. A. (2015). Developmental Cognitive Neuroscience Is " efficiency " a useful concept in cognitive neuroscience ? Developmental Cognitive Neuroscience, 11, 12-17. http://doi.org/10.1016/j.dcn.2014.06.001

Postle, B. R. (2006). Working memory as an emergent property of the mind and brain. Neuroscience, 139(1), 23-38. http://doi.org/10.1016/j.neuroscience.2005.06.005

Redick, T.S., \& Lindsey, D. R. B. (2013). Complex span and n-back measures of working memory: A meta-analysis. Psychonomic Bulletin \& Review, 20(6), 1102-1113. http://doi.org/10.3758/s13423-013-0453-9

Redick, T. S., Shipstead, Z., Harrison, T. L., Hicks, K. L., Fried, D. E., Hambrick, D. Z., ... Engle, R. W. (2013). No Evidence of Intelligence Improvement After Working Memory Training: A Randomized, Placebo-Controlled Study. Journal of Experimental Psychology: General, 142(2), 359-379. http://doi.org/10.1037/a0029082

Reuter-Lorenz, P. A., \& Cappell, K. A. (2008). Neurocognitive Aging and the Compensation Hypothesis. Current Directions in Psychological Science, 17(3), 177-182. http://doi.org/10.1111/j.1467-8721.2008.00570.x 
Salminen, T., Strobach, T., \& Schubert, T. (2012). On the impacts of working memory training on executive functioning. Frontiers in Human Neuroscience, 6(June), 1-14. http://doi.org/10.3389/fnhum.2012.00166

Schneiders, J. a, Opitz, B., Krick, C. M., \& Mecklinger, A. (2011). Separating intra-modal and across-modal training effects in visual working memory: an fMRI investigation. Cerebral Cortex (New York, N.Y.: 1991), 21(11), 2555-64. http://doi.org/10.1093/cercor/bhr037

Schneiders, J., Opitz, B., Tang, H., Deng, Y., Xie, C., Li, H., \& Mecklinger, A. (2012). The impact of auditory working memory training on the fronto-parietal working memory network. Frontiers in Human Neuroscience, 6(June), 173. http://doi.org/10.3389/fnhum.2012.00173

Schweizer, S., Grahn, J., Hampshire, A., Mobbs, D., \& Dalgleish, T. (2013). Training the emotional brain: Improving affective control through emotional working memory training. Annals of Internal Medicine, 158(6), 5301-5311. http://doi.org/10.1523/JNEUROSCI.2593-12.2013

Smith, E. E., \& Jonides, J. (1999). Storage and executive processes in the frontal lobes. Science (New York, N.Y.), 283(5408), 1657-61. http://doi.org/http://dx.doi.org/10.1016/B978-0-12-385157-4.01148-9

Takeuchi, H., Sekiguchi, A., Taki, Y., Yokoyama, S., Yomogida, Y., Komuro, N., ... Kawashima, R. (2010). Training of Working Memory Impacts Structural Connectivity. Journal of Neuroscience, 30(9), 3297-3303. http://doi.org/10.1523/JNEUROSCI.4611-09.2010

Thompson, T. W., Waskom, M. L., \& Gabrieli, J. D. E. (2016). Intensive Working Memory Training Produces Functional Changes in Large-scale Frontoparietal Networks. Journal of Cognitive Neuroscience, 28(4), 575-88. http://doi.org/10.1162/jocn_a_00916

Thompson, T. W., Waskom, M. L., Garel, K. L. A., Cardenas-Iniguez, C., Reynolds, G. O., Winter, R., ... Gabrieli, J. D. E. (2013). Failure of Working Memory Training to Enhance Cognition or Intelligence. PLoS ONE, 8(5). http://doi.org/10.1371/journal.pone.0063614

Vandervert, L. (2009). WorkingMemory, the Cognitive Functions of the Cerebellum and the Child Prodigy. In: L. Shavinina, ed., International Handbook on Giftedness, 1st ed. pp.3-13. Springer Science+Business Media B.V. doi: 10.1007/978-1-4020-6162213

Vartanian, O., Jobidon, M.-E., Bouak, F., Nakashima, A., Smith, I., Lam, Q., \& Cheung, B. (2013). Working memory training is associated with lower prefrontal cortex activation in a divergent thinking task. Neuroscience, 236, 186-194. http://doi.org/10.1016/j.neuroscience.2012.12.060

von Bastian, C. C., \& Oberauer, K. (2014). Effects and mechanisms of working memory training: a review. Psychological Research, 78(6), 803-820. http://doi.org/10.1007/s00426-013-0524-6

Wager, T.D., \& Smith, E. E. (2003). Neuroimaging studies of working memory: A metaanalysis. Cognitive, Affective and Behavioral Neuroscience, 3(4), 255-274. Retrieved from http://www.scopus.com/inward/record.url?eid=2-s2.02342597739\&partnerID=40\&md5=d13bba90ed9b65ae16fd931accf885b1

Watanabe, Y., \& Funahashi, S. (2012). Thalamic mediodorsal nucleus and working memory. Neuroscience and Biobehavioral Reviews, 36(1), 134-42. http://doi.org/10.1016/j.neubiorev.2011.05.003

Westerberg, H., \& Klingberg, T. (2007). Changes in cortical activity after training of working memory - a single-subject analysis. Physiology and Behavior, 92(1-2), 
186-192. http://doi.org/10.1016/j.physbeh.2007.05.041

Woo, C.-W., Krishnan, A., \& Wager, T. D. (2014). Cluster-extent based thresholding in fMRI analyses: pitfalls and recommendations. NeuroImage, 91, 412-9. http://doi.org/10.1016/j.neuroimage.2013.12.058

Yamashita, M., Kawato, M., \& Imamizu, H. (2015). Predicting learning plateau of working memory from whole-brain intrinsic network connectivity patterns. Scientific Reports, 5, 7622. http://doi.org/10.1038/srep07622

Zou, Q., Ross, T. J., Gu, H., Geng, X., Zuo, X.-N., Hong, L. E., ... Yang, Y. (2013). Intrinsic resting-state activity predicts working memory brain activation and behavioral performance. Human Brain Mapping, 34(12), 3204-15. http://doi.org/10.1002/hbm.22136

\section{SUPPORTING INFORMATION}

Test-retest reliability analyses for behavioral and imaging control group data:

\section{Behavioral test-retest reliability analyses}

We calculated the re-test correlations separately for accuracy and reaction times (RTs).

The results yielded significant positive correlations: Session $1-$ Session 2: $r_{27}=0.53$ $\mathrm{p}<0.05$; Session $1-$ Session 3: $\mathrm{r}_{27}=0.54 \mathrm{p}<0.05$ and Session $2-$ Session 3: $\mathrm{r}_{27}=0.71$ $\mathrm{p}<0.01$ for accuracy and Session $1-$ Session 2: $\mathrm{r}_{27}=0.88 \mathrm{p}<0.01$; Session $1-$ Session 3: $\mathrm{r}_{27}=0.86 \mathrm{p}<0.01$ and Session $2-$ Session 3: $\mathrm{r}_{27}=0.95 \mathrm{p}<0.01$ for RTs.

\section{Imaging test-retest reliability analyses}

For imaging data, we performed three one sample $t$ tests (one per session) in order to observe the reliability. Here, we present the results in a figure: 
Supplementary Figure: One Sample $t$ test for the control group in (a) Session 1, (b) Session 2, and (c) Session 3, showing the $n$-back general activations. The same contrast was utilized for the 3 sessions: 2-back+3-back $>0$-back. Results were $p<.05$ FWE-cluster corrected with cluster criteria of $k=657$ voxels for Session $1, k=101$ for Session 2, and $k=161$ voxels for Session $3 . \mathrm{L}=$ left and $\mathrm{R}=$ right.

a

z: 6

z: 30

z: 40

z: 50

$\mathrm{L}$

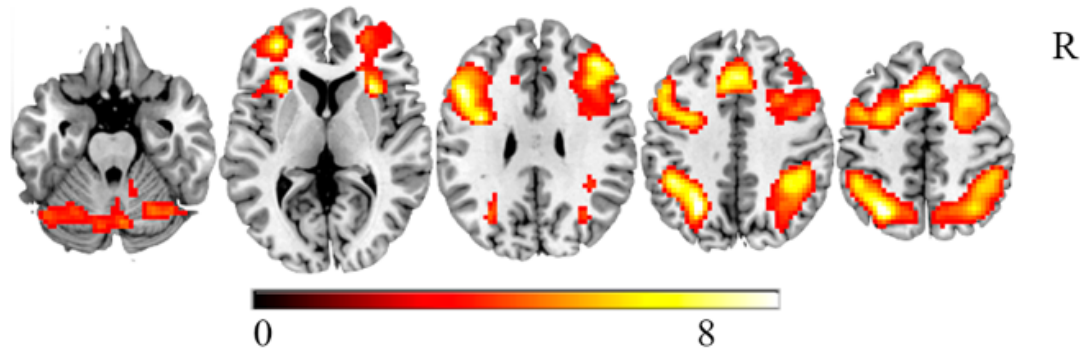

b

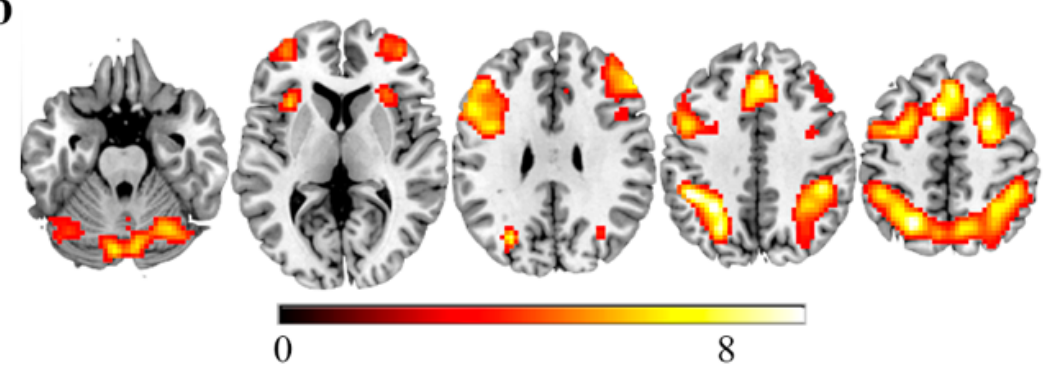

c

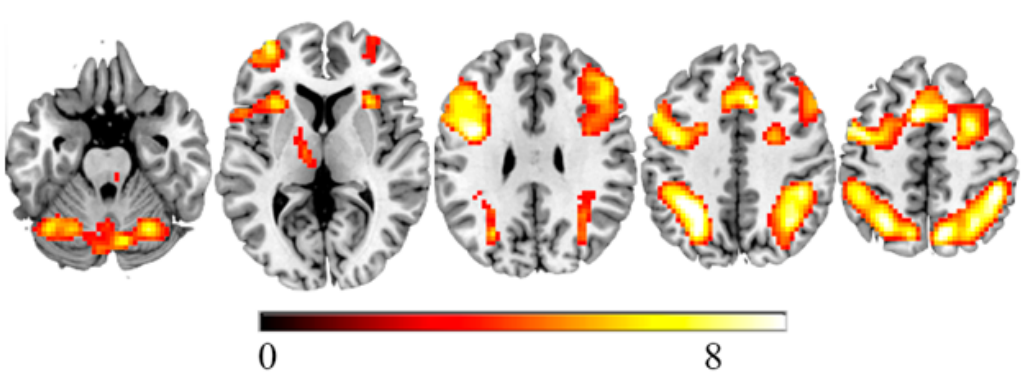




\begin{abstract}
Study 2
Locating neural transfer effects of N-back training in the central executive: a longitudinal fMRI study
\end{abstract}

Anna Miró-Padilla, Elisenda Bueichekú and César Ávila

Under review 


\begin{abstract}
The large number of behavioral studies testing whether working memory training improves performance on an untrained task have yielded inconclusive results. Moreover, some studies have investigated the possible neural changes during the performance of untrained tasks after training. Here, we studied the far transfer from $n$-back training to the PASAT test, two different tasks that use the central executive to maintain verbal stimuli. Participants completed fMRI sessions at baseline, immediately after one week of training, and at the five-week follow-up. Although behavioral transfer effects were not obtained, training was associated with decreased activation in the anterior bilateral DLPFC (BA 9/46) while performing the PASAT that remained stable five weeks later. In addition, this decline was positively associated with behavioral improvements on the PASAT test. Consistent with our hypothesis, the changes in the anterior DLFPC largely overlapped with the $n$-back task fMRI activations. In conclusion, working memory training improves efficiency in brain areas involved in the trained task that may affect untrained tasks, specifically in brain areas responsible for the same cognitive processes.
\end{abstract}

\title{
KEYWORDS
}

fMRI; working memory training; transfer effects; long-term effects; PASAT; $n$-back 


\section{INTRODUCTION}

The effects of cognitive training have become a popular research topic. Specifically, in recent years, working memory training has been studied by a large number of researchers, but with no agreement about its behavioral and neural effects on untrained tasks. Although it seems clear that working memory training leads to improvements in the trained tasks (see e.g. Klingberg, 2010; Morrison \& Chein, 2011; von Bastian \& Oberauer, 2014 for reviews), the controversy arises in relation to the possible transfer effects to untrained tasks. In this field, the distinction between near and far transfer is relevant. Near and far transfer depend on the structural similarity between the trained and untrained tasks (Karbach \& Kray, 2009; Karbach \& Verhaeghen, 2014; Melby-Lervåg, Redick, \& Hulme, 2016): near transfer is produced between structurally similar or identical tasks, and far transfer occurs between dissimilar working memory tasks. Some studies have reported near and far transfer effects (Buschkuehl, Hernandez-Garcia, Jaeggi, Bernard, \& Jonides, 2014; Jaeggi, Buschkuehl, Jonides, \& Shah, 2011; Jaeggi et al., 2010b; Schweizer, Grahn, Hampshire, Mobbs, \& Dalgleish, 2013), others have only found near transfer effects (Harrison et al., 2013; Minear et al., 2016; Salminen, Strobach, \& Schubert, 2012; Waris, Soveri, \& Laine, 2015), and still others did not find any kind of transfer (Chooi \& Thompson, 2012; Clark, Lawlor-Savage, \& Goghari, 2017a; Redick \& Lindsey, 2013; Thompson et al., 2013). At least five meta-analyses (Au, Buschkuehl, Duncan, \& Jaeggi, 2015a; Au, Sheehan, et al., 2015b; Melby-Lervåg \& Hulme, 2016; Melby-Lervåg et al., 2016; Soveri, Antfolk, Karlsson, Salo, \& Laine, 2017) have been conducted to clarify the possible transfer effects in the working memory domain, but the overall results have been inconclusive. On the one hand, Au et al. (2015a; 2015b) found support for near and far transfer effects. Two other studies found moderate near transfer effects, but they concluded that there are no convincing far transfer effects (Melby-Lervåg 
\& Hulme, 2016; Melby-Lervåg et al., 2016). Meanwhile the most recent review by Soveri et al. (2017) reported a moderate effect of task-specific transfer, but very small near and far transfer effects. Furthermore, this last study did not find a moderating role in the results of the kind of control group, the training type, the number of sessions, or the hours of training. Importantly, all these meta-analyses examined studies that used the $n$-back task for their working memory training. Therefore, other non-behavioral approaches should be used to investigate transfer effects after working memory training.

Working memory is mainly supported by a distributed frontoparietal set of cortical regions (Constantinidis \& Klingberg, 2016). The intraparietal and superior parietal cortex and the posterior parts of the superior frontal gyrus are more involved in visuospatial working memory tasks, whereas the more anterior parts of the prefrontal cortex act as the central executive and are crucial for storing non-spatial information and exerting topdown control over posterior regions. The top-down control exerted by the anterior parts of the prefrontal cortex has been observed across a variety of cognitive tasks, independently of the complexity or cognitive demands (Duncan \& Owen, 2000; Edin et al., 2009).

Apart from the vast number of working memory behavioral studies, there is little research examining the cerebral changes produced by working memory training. Recent scientific reviews on this topic describe the brain changes after different kinds of training (Buschkuehl, Jaeggi, \& Jonides, 2012; Constantinidis \& Klingberg, 2016). Again, disagreement is found because both increases and decreases in task-related BOLD activity have been described after training in working memory brain related areas, especially in frontoparietal areas. However, in the specific case of $n$-back training, 
Buschkuehl et al. (2012) concluded that all studies showed decreased activation after training in the areas involved in the $n$-back task, which are the right superior middle frontal gyrus (BA 6), posterior parietal regions (BA 40), and dorsolateral prefrontal cortex (DLPFC; BA 9, 46). Thus, the effect of $n$-back training on efficiency on the same task leads to improved performance and a reduction of the activation in frontoparietal areas.

Research focused on the study of near and far transfer effects in the brain is still scarce (Chang et al., 2017; Clark, Lawlor-Savage, \& Goghari, 2017b; Dahlin, Neely, Larsson, Bäckman, \& Nyberg, 2008; Salminen, Kühn, Frensch, \& Schubert, 2016). In a recent study that compared HIV patients to healthy controls, participants trained on an adaptive $n$-back task (20 to 25 sessions for 5-8 weeks) and completed three fMRI sessions (baseline, 1-month and 6-month follow-up sessions), performing 1- and 2-back tasks (Chang et al., 2017). The results showed improvements in performance and decreased activation in frontoparietal areas in both groups at the follow-up sessions. Importantly, the decreased activation observed in the DLPFC while performing the 2-back task correlated with behavioral improvements on a short-term memory task (i.e. Digit Span task, performed outside the scanner) in the HIV group, whereas both groups had decreases in activation in the middle frontal cortex during the 1-back task that correlated with shortterm behavioral improvements. Therefore, the decreased activation in frontal areas on the trained task was related to near transfer. However, another very recent study did not replicate these results when transfer effects to a fluid intelligence test were studied (Clark et al., 2017b). The authors confirmed that $n$-back training decreased brain activation on the task, but it did not produce any kind of neuroplasticity changes while performing Raven's Matrix test. In Dahlin et al. (2008), participants engaged in five weeks of computer-based updating training on a letter memory task requiring the discrete updating 
of four letters. $\mathrm{N}$-back and Stroop tests were included as transfer tasks. Analyses of posttraining changes in fMRI data during the performance of the letter memory task showed increased activation in the left striatum, temporal, and occipital regions, but also decreased activity in frontal and parietal areas. The near transfer effects to the $n$-back task were seen in the left striatum cortex, and no significant transfer effects to the Stroop task were found. The authors confirmed the hypothesis that transfer effects occur when the training task and the transfer task involve the same brain regions and processing components. A more recent study found increases in the occipital cortex and the striatum while performing a near transfer task, even on trained and untrained tasks requiring the updating of working memory (Salminen et al., 2016). Importantly, the neural transfer was observed in the dual-task condition, but not in the single task conditions because it was specific to the process of updating two different stimuli.

The aim of this study was to investigate neural far transfer processes between two structurally different working memory tasks: the $n$-back and the Paced Auditory Addition Test (PASAT). Both tasks are common experimental working memory paradigms that continuously require keeping information available for subsequent complex processing (Jaeggi, Buschkuehl, Perrig, \& Meier, 2010a; Owen, McMillan, Laird, \& Bullmore, 2005; Tombaugh, 2006), and both require the participation of the DLPFC (Audoin et al., 2003; Forn et al., 2011; Owen et al., 2005). They also have the premotor cortex, the dorsal anterior cingulate cortex, and the posterior parietal cortex (Audoin et al., 2003; Correia, 2011; Forn et al., 2011; Owen et al., 2005) in common during the execution of the task. Both tasks require the person to maintain and update past information, but with different posterior cognitive processes involved: the $n$-back task requires the subject to maintain 
letters, whereas the PASAT requires him/her to maintain a number, resist the interference of a generated number, and add it to the actual number.

Through a longitudinal fMRI study, the present study investigated the possible transfer effect from $n$-back training to PASAT at the behavioral and brain levels. In addition, a follow-up session was included to study the stability of the changes (if there were any), in order to obtain valuable information about the effect of training over time. Our hypothesis is that neural transfer would involve reduced activation in the DLPFC, which would mediate top-down control on both tasks. The DLPFC is the main brain area involved in non-spatial working memory processing (Constantinidis \& Klingberg, 2016), and it has been involved in transfer effects in previous research (Chang et al., 2017). Posterior brain areas were not expected to be sensitive to transfer effects because they have different roles in the two tasks (maintain letters in memory vs add/manipulate numbers).

\section{MATERIALS AND METHODS}

\section{Participants}

In the present study, 52 healthy right-handed undergraduates (20 male) participated. All of them were recruited from the student population of the Universitat Jaume I, and none of them reported a previous psychiatric or neurologic diagnosis. The Ethical Committee of the Universitat Jaume I approved the research project, and informed consent was obtained from each subject before participation. They received monetary compensation for their active participation. Participants were randomly assigned to either an experimental condition (trained group) $(\mathrm{N}=25$, mean age $=22.72 \pm 1.51,10$ men $)$ or a control condition (control group) $(\mathrm{N}=27$, mean age $=22.52 \pm 1.45,10$ men). Their 
intellectual level was assessed with the Matrix Reasoning Test (WAIS-III-R) (trained group: mean $=21.08 \pm 3.35$; control group: mean=21.63 \pm 1.94$)$. Between-group differences in gender distribution, age, and IQ were non-significant. The two groups differed only on the training (the control group did nothing).

\section{Experimental design}

Both groups completed three fMRI sessions with the same adapted block-design PASAT test. The second fMRI session took place seven days after the first one, and the last session took place 35 days (five weeks) after the second session. The trained group trained 200 minutes on an adaptive $n$-back task between the two first scanner sessions. Neither group had training between the two last sessions. The pre-training session, post-training session, and follow-up session correspond to Session one (S1), Session two (S2), and Session three (S3), respectively. Auditory stimuli (numbers) were presented electronically using E-Prime software (Psychology Software Tools, Pittsburgh, PA), professional version 2.0, installed in a Hewlett-Packard portable workstation (screen-resolution 800 x 600, refresh rate of $60 \mathrm{~Hz}$ ). Participants listened to the stimuli through MRI-compatible headphones (VisuaStim, Resonance Technology, Inc., Northridge, CA, USA), and their responses were recorded via an MRI-compatible microphone (FORMIII, Optoacoustics, Inc.). Sound volume was adjusted so that each participant could hear the stimuli properly, and scanner noise was cancelled.

\section{PASAT fMRI task}

Participants completed six-minute versions of the auditory PASAT task, which included six one-minute blocks. Three of these six PASAT blocks belonged to the control condition (repeat), and three belonged to the activation task (add). The subjects heard a 
sequence of numbers, ranging from one to nine, at a rate of one number every three seconds (19 stimuli per block). During the control task, participants were instructed to repeat each number in a presented series aloud. The activation task consisted of adding the first number to the second, the second to the third, and so on. They calculated the sum of the last two numbers and responded aloud (Forn et al., 2006). We collected the number of correct responses; 54 was the maximum accuracy score per task (18 per block). Participants were asked to answer accurately and try not to make mistakes, and they received oral instructions about how to do the task. In order to become familiar with the stimuli presentation and how to respond, they performed a four-minute practice task outside the scanner that was composed of four blocks, two per condition. A similar laptop was used with the same display features and the same hardware for spoken responses.

\section{N-back training task}

As described in our previous study (Miró-Padilla et al., 2018), the trained group carried out four consecutive training sessions (TS) of single $n$-back after fMRI in S1 in our laboratory located at the University. Participants performed only one TS per day. One TS lasted 60 minutes and was distributed in two phases: the learning part and the testing part. In the learning part, participants performed an adaptive $n$-back paradigm adapted from (Jaeggi, Buschkuehl, Jonides, \& Perrig, 2008) for 50 minutes, whereas in the testing part, they performed a simple $n$-back task that lasted 10 minutes. Therefore, the total training time was approximately 200 minutes, plus 40 minutes for the testing part. This specific training was chosen because it has been demonstrated that in short periods of training, single $n$-back training is more effective than dual $n$-back training (Küper \& Karbach, 2016). 
For the adaptive $n$-back task, three active load levels (1-, 2- and 3-back) were presented, and participants pressed the "yes" button when the current letter shown on the screen matched the one presented 1, 2 or 3 items back, and they pressed "no" when there were no targets. Participants were given feedback about their performance after each stimulus and at the end of each block. The task lasted approximately 16 minutes, and subjects performed three runs per TS. Each block lasted 60.7 seconds and consisted of $200 \mathrm{~ms}$ of a blank screen, followed by 30 (6 target) consecutive trials of single letter stimuli (500 ms duration, $1500 \mathrm{~ms}$ inter-stimulus interval) with $500 \mathrm{~ms}$ of a blank screen at the end of each block. In addition, $8000 \mathrm{~ms}$ of a fixation cross and $2000 \mathrm{~ms}$ of an instruction display indicating task difficulty (1-back, 2-back or 3-back) were included before each block. The sequence of the stimuli was pseudo-randomized. The visual material comprised 15 different capital letters from the alphabet (B, C, D, F, G, H, J, L, N, P, Q, R, S, T and V). Any letter could be a target, and the task did not contain any lures. The letters, the instructions, and the fixation point were presented on the middle of the screen on a white background. All were in black ink with a 54-point Arial font. Subjects had to give manual responses with only their right hand, responding to targets with their thumb and to nontargets with their forefinger. Participants were asked to answer accurately and as quickly as possible.

In order to motivate participants to improve, we changed the level of difficulty by changing the level of " $n$ " $(1,2$, or 3$)$. After each block, the participant's individual performance was analyzed, and the $n$-back level was automatically adjusted up to a maximum of 3-back. Thus, if the participant had at least $90 \%$ correct answers, the level of " $n$ " in the next block was increased by one, but it was decreased by one if accuracy was below $80 \%$. In all other cases, the n-level remained constant. In the last run, we 
increased the percentage by five percent to make it more difficult. Therefore, if the participant had at least $95 \%$ correct answers, the level of "n" was increased by one, whereas it was decreased by one if accuracy was below $85 \%$. Each run started with the minimum level of "n" (1) for motivational reasons. Feedback was introduced after each response: a colored circle appeared for a few seconds at the corner of the screen: green if the answer was correct, red if it was an error, and blue if participants did not press any button. Furthermore, at the end of each block, subjects received information about their performance: correct response percentage and reaction time average.

On the test part, participants performed an eight-block $n$-back task, four for 2-back and four for the 3-back load level; the entire task lasted 10 minutes. Subjects had no feedback this time. The E-Prime logfile saved each participant's accuracy and reaction time (RT) to each stimulus. Their results on this test were useful to evaluate their progress on the task. We used the same laptop as in the fMRI sessions, and their responses were collected via response-grips (NordicNeuroLab, Bergen, Norway).

\section{Neuroimaging data acquisition and preprocessing}

Functional MRI data were collected on a 1.5T Siemens Symphony scanner (Erlangen, Germany). The same sequences were used in the three sessions. Participants were placed in a supine position in the MRI scanner, and their heads were immobilized with cushions to reduce motion degradation. Furthermore, participants were asked to minimize head movement, even while giving the answers. For task-fMRI, a gradient-echo T2*-weighted echo-planar MR sequence covering the entire brain was used $(\mathrm{TR} / \mathrm{TE}=2500 / 49 \mathrm{~ms}$, matrix $=64 \times 64 \times 28$, flip angle $=90^{\circ}$, voxel size $=3.5 \times 3.5 \times 4.48$; slice thickness $=4 \mathrm{~mm}$; slice gap $=0.48 \mathrm{~mm})$. A total of 146 volumes were recorded. The slices were made parallel to the anterior-posterior commissure plane covering the entire brain. Before the 
fMRI sequences, a high-resolution structural T1-weighted MPRAGE sequence was acquired $\left(\mathrm{TR}=2200 \mathrm{~ms} ; \mathrm{TE}=3 \mathrm{~ms}\right.$; flip angle $90^{\circ}$, matrix $=256 \times 256 \times 160$; voxel size $=1 \times 1 \times 1 \mathrm{~mm})$.

Preprocessing and statistical analyses of fMRI data were conducted with SPM12 (Wellcome Trust Centre for Neuroimaging, London, UK). We aligned each subject's fMRI data to the AC-PC plane by using his/her anatomical image. The fMRI preprocessing included head motion correction, where the functional images were realigned and resliced to fit the mean functional image. No participant had a head motion of more than $2.5 \mathrm{~mm}$ maximum displacement in any direction or $2.5^{\circ}$ of any angular motion throughout the scan. Afterwards, the anatomical image (T1-weighted) was coregistered to the mean functional image, and the transformed anatomical image was then re-segmented. The functional images were spatially normalized to the MNI (Montreal Neurological Institute, Montreal, Canada) space with $3 \mathrm{~mm} 3$ resolution, and spatially smoothed with an isotropic Gaussian kernel of $8 \mathrm{~mm}$ FWHM (Full-Width at HalfMaximum).

\section{Behavioral analysis}

To process the behavioral data (accuracy for participants' performance), IBM SPSS Statistics software (Version 22 Armonk, New York, USA) was used. A repeatedmeasures 2x3 mixed-model ANOVA was conducted for each variable, using Group (training $\mathrm{x}$ control) as the between-subject factor and Session (1 vs. 2 vs. 3 ) as the withinsubject factors. With the data from the test part of the training, a repeated-measures $2 \times 4$ ANOVA was conducted, with Load Level (2-back vs. 3-back) and Training Session (1 vs. 2 vs. 3 vs. 4) as within-subject factors. 
Neuroimaging analysis

fMRI-task post-processing analysis: First level of analysis

Statistical analyses were performed in the context of the General Linear Model (Friston et al., 1995) for each participant and for each time point, using SPM12. In the first-level analysis, we modeled the conditions of interest corresponding to add $>$ repeat (active condition > control condition). The BOLD signal was estimated by convolving the stimuli onset with the canonical hemodynamic response function. Six motion realignment parameters were included to explain signal variations due to head motion, that is, as covariates of no interest. A high-pass filter (128s) was applied to the functional data to eliminate low-frequency components. Then, contrast images were obtained to directly compare our conditions of interest. For the cross-sectional analysis, the S1 conditions of interest were compared in order to assess differences on the PASAT task before learning.

\section{fMRI-task post-processing analysis: Statistical analysis}

In the cross-sectional analysis, a whole-brain one-sample $t$ test was conducted in order to study the brain regions involved in the PASAT task (active condition > control condition) using the fMRI data collected in S1. In addition, first session data were used to perform a two-sample $t$ test to examine the equality of the brain responses in the two groups. Therefore, any between-group brain differences found in subsequent sessions would be due to training effects. In the second-level analysis, the longitudinal analysis was performed, with an interaction analysis between sessions to evaluate: the immediate effect of training, comparing S2 to S1; the long-term effects of training, comparing S3 to $\mathrm{S} 1$; and the effects of differences between immediate and long-term effects, comparing S3 to S2. Additionally, a conjunction analysis was performed for both groups using S1 data in order to identify the brain regions that are commonly activated by the training task 
( $n$-back; contrast: 2 -back +3 back $>0$-back) and the transfer task (PASAT; contrast: add>repeat). The $n$-back data were obtained from our previous study (Miró-Padilla et al., 2018) of the same participants who completed the present study. To avoid false positives in the fMRI analyses (Woo, Krishnan, \& Wager, 2014), the statistical criterion used in all the fMRI analyses was set at $\mathrm{p}<0.05$, and family-wise error (FWE) was cluster-corrected for multiple comparisons (voxel-level uncorrected threshold of $\mathrm{p}<0.001$; specific cluster sizes appear in each result).

In addition, the association between the improvement in scores on the PASAT task and the changes in brain activation was tested using the definition of ROIs with a size of $6 \mathrm{~mm}$ extracted from regions with significant changes after the training. The first eigenvariate of each ROI was extracted as a measure of its average signal across participants, using a VOI command of SPM12. This method uses the temporal covariance of voxels in the ROI to find coherent spatial modes of activity (Friston et al., 1995), and it provides a summary of the responses within an ROI. Finally, to study the brain-behavior relationships, linear bivariate Pearson's correlation analyses of the data extracted from the brain regions were conducted using IBM SPSS Statistics software, and they were correlated with the PASAT and training improvement scores. To obtain the performance improvement scores, we calculated the difference in accuracy (number of correct answers) from S1 to S2 on the PASAT and from TS1 to TS4 on the 2-back and 3-back training tasks. 


\section{RESULTS}

$\underline{\text { Behavioral results }}$

Behavioral fMRI results

Mean performance on the pre-training was close to $85 \%$ for both groups (Figure 1). The repeated-measures $2 \times 3$ mixed-model ANOVA conducted for accuracy on the PASAT test yielded main effects for Session $(\mathrm{F}(2,48)=7.72 \mathrm{p}=.001)$, indicating improved performance in post-training sessions. Importantly, the main effect of Group and the Group $\mathrm{x}$ Session interaction were non-significant, showing that the trained group did not perform the task significantly better than the control group after $n$-back training.

Figure 1: Results of the PASAT behavioral analysis, differences were found between sessions, but not between groups. Correct-responses percentage. Pre-training session, post-training session, and follow-up session correspond to Session 1 (S1), Session 2 (S2), and Session 3 (S3), respectively. Trained group data correspond to the dark bars, and control group data to the light bars. Error bars represent standard error. $\mathrm{N}=52$ (Trained group $=25$; Control group $=27$ ).

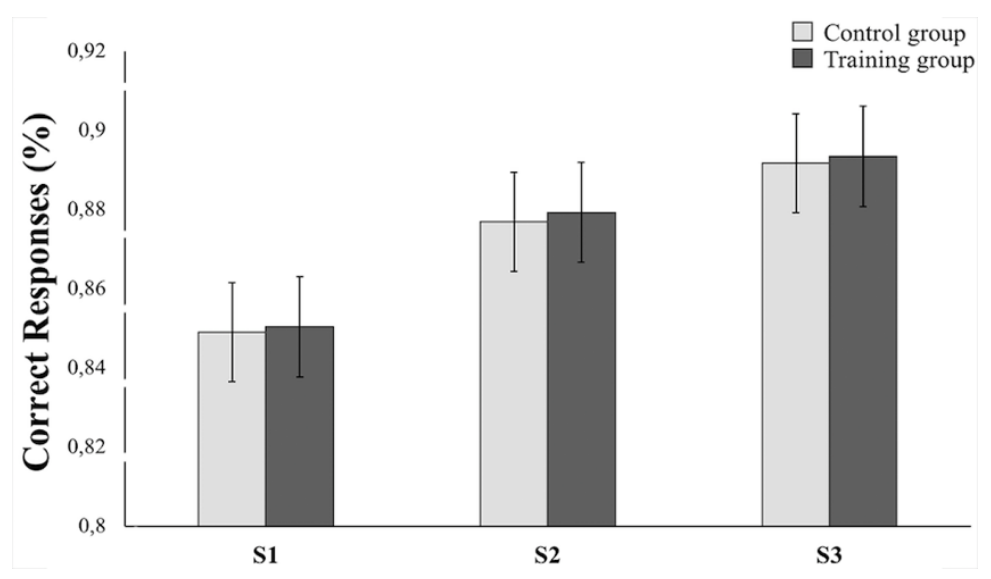

\section{Behavioral training results}

These behavioral data have been reported in our previous study (Miró-Padilla et al., 2018), but we are going to describe them briefly to emphasize the progress of the trained group on $n$-back during the training sessions. The results of the test part of the training 
were used, with the correct responses and reaction times (RTs), and a repeated-measures 2x4 ANOVA was performed. Regarding accuracy, the ANOVA showed significant effects of the Training Session $(F(3,22)=4.85 \mathrm{p}<.05)$, the Load Level $(\mathrm{F}(1,24)=8.06$ $\mathrm{p}<.05)$, and the Load Level $\mathrm{x}$ Training Session interaction $(\mathrm{F}(3,22)=4.07 \mathrm{p}<.05)$. As expected, the performance on the $n$-back task improved significantly and progressively after each training session on both the 2-back and 3-back tasks. Regarding the RTs values, results showed a significant effect of Training Session $(F(3,22)=8.62 \mathrm{p}<.001)$, which means that subjects' RTs decreased significantly from one training session to another. The overall results indicate that trained participants steadily improved their accuracy as they accumulated 200 minutes of training.

\section{$\underline{\text { Task fMRI results }}$}

\section{Task effects at baseline}

A whole-brain one-sample t test (active condition $>$ control condition) was used to study the brain regions that support the performance on the PASAT task; for this purpose, the data collected in the pre-training session (S1) were used. Activations were found in cortical and subcortical areas related to working memory processes. Specifically, the results yielded activations in the bilateral middle and inferior frontal cortex (BA 6/10/4446), including the anterior insula (BA13), bilateral superior and inferior parietal cortex (BA 7/40), bilateral inferior temporal cortex (BA 20), and bilateral cerebellum. The activated midbrain areas were the thalamus and the caudate (Figure 2). Results were collapsed across groups, and $\mathrm{p}<.05$ FWE, cluster-corrected using a threshold of $\mathrm{p}<.001$ at the uncorrected voxel level and a minimum cluster extension of $\mathrm{k}=110$ voxels. 
Figure 2: PASAT general task activations in the pre-training session. Contrast: $a d d>$ repeat (active condition $>$ control condition). Results were $\mathrm{p}<.05$ FWE clustercorrected using a threshold of $\mathrm{p}<.001$ at the uncorrected voxel level and a cluster extension of $\mathrm{k}=110$ voxels.

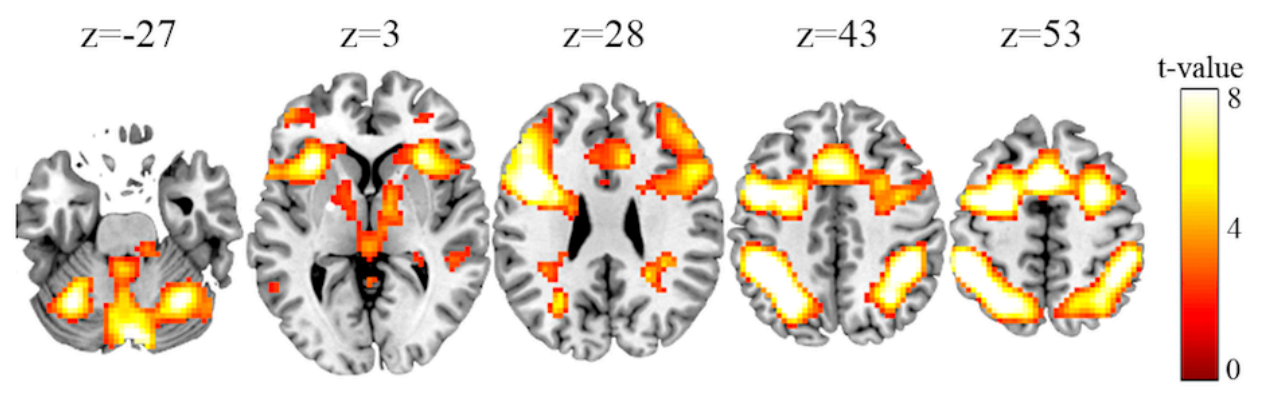

In addition, the data collected in $\mathrm{S} 1$ were used to perform a two-sample $\mathrm{t}$ test analysis in order to verify that the two groups had no differences in brain responses during the PASAT performance. The results showed no significant functional differences at the $\mathrm{p}<0.05$ FWE, cluster-corrected threshold.

\section{Neural Transfer effects: Group x Session interaction analysis results}

The aim of the interaction analyses was to study the training effects on the training participants and across sessions while controlling for repetition effects by using betweensubjects controls. These analyses assessed the brain changes from $\mathrm{S} 1$ to $\mathrm{S} 2$; and $\mathrm{S} 3$, in terms of increases or decreases in cerebral activation. When studying the effects of the trained group [contrasts: (1) Trained group $(\mathrm{S} 1>\mathrm{S} 2)>$ Control group $(\mathrm{S} 1>\mathrm{S} 2)$ and (2) Trained group $(\mathrm{S} 1>\mathrm{S} 3)>$ Control group $(\mathrm{S} 1>\mathrm{S} 3)]$, the trained participants had less activation in the bilateral DLPFC than the control participants during S2 and S3, compared to the pre-training session (see Figure 3 and Table 1). The opposite contrasts yielded no significant effects; in other words, no increased activations were found across sessions in trained participants compared to controls. Results were $\mathrm{p}<.05$ FWE cluster- 
corrected using a threshold of $\mathrm{p}<.001$ at the uncorrected voxel level and a minimum cluster extension of $\mathrm{k}=70$ voxels and $\mathrm{k}=99$ voxels, respectively.

Figure 3: Decreased activation was found in the prefrontal lobe during the performance of the PASAT task in the trained group after the $n$-back training. (A) represents the contrast: Trained group $(\mathrm{S} 1>\mathrm{S} 2)>$ Control group $(\mathrm{S} 1>\mathrm{S} 2)$; and (B) represents the contrast: Trained group $(\mathrm{S} 1>\mathrm{S} 3)>$ Control group $(\mathrm{S} 1>\mathrm{S} 3)$. Results were $\mathrm{p}<.05$ FWE clustercorrected using a threshold of $\mathrm{p}<.001$ at the uncorrected voxel level and a cluster extension of $\mathrm{k}=70$ voxels and $\mathrm{k}=99$ voxels, respectively.

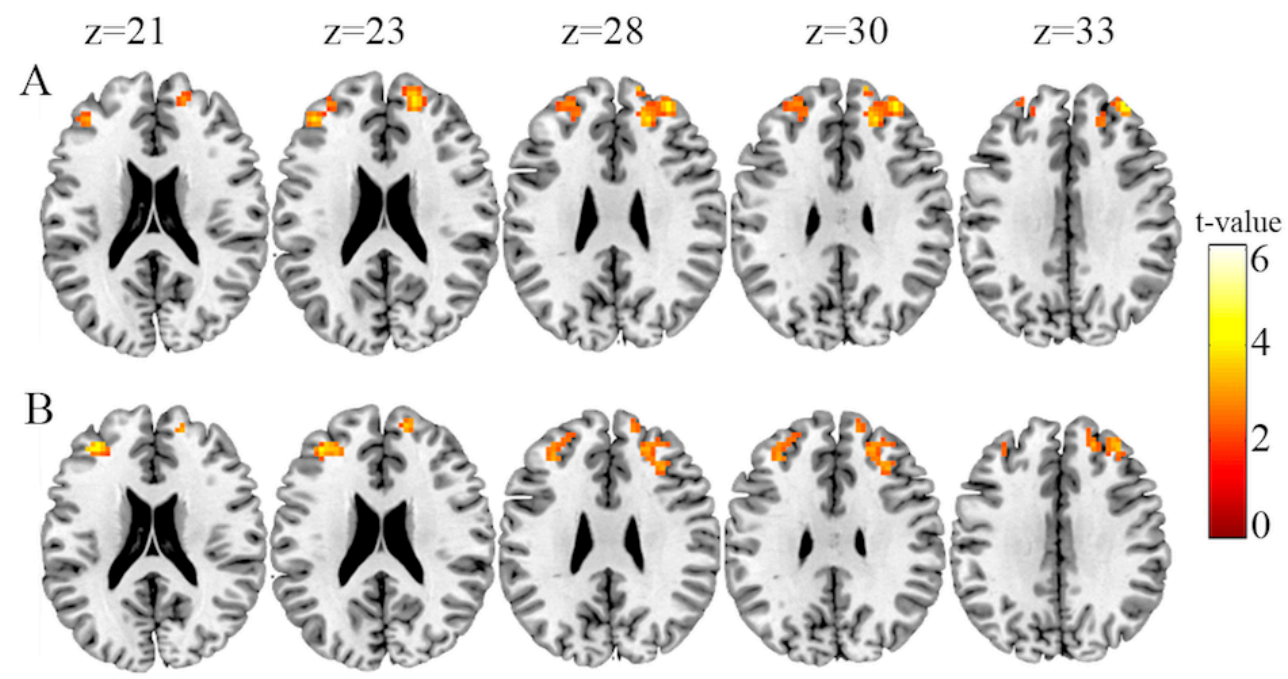

Neural Transfer effects: stability of the effects

A similar Group x Session interaction analysis was also conducted in order to study the stability of the effects of the working memory training over time. After five weeks of no training or contact with the training task, trained participants could have lost the training effects and returned to their baseline brain response (which would show more activation in S3 than in S2) or maintained the training effects (which would show no differences between S2 and S3). Therefore, the contrast used to study the stability of the effects was: Trained group (S2 vs. S3) > Control group (S2 vs. S3). No significant effects were found 
in either condition or any comparison; that is, the decreased activation found from $\mathrm{S} 1$ to $\mathrm{S} 2$ remained stable in S3 without additional changes.

Table 1: Interaction analysis results: A) comparing pre-training (S1) to post-training session (S2) and B) comparing pre-training (S1) to follow-up session (S3). The MNI coordinates described here were used to extract the ROIs for correlation analysis.

\begin{tabular}{|c|c|c|c|c|c|c|}
\hline & \multirow[b]{2}{*}{$\mathbf{B A}$} & \multicolumn{5}{|c|}{ MNI SPACE } \\
\hline & & Cluster extent & $\mathbf{x}$ & $\mathbf{y}$ & $\mathbf{z}$ & Z-value \\
\hline \multicolumn{7}{|c|}{ A) Trained Group $(\mathrm{S} 1>$ S2) $>$ Control Group $(\mathrm{S} 1>$ S2) } \\
\hline R DLPFC & 46 & 113 & 33 & 47 & 29 & 4.27 \\
\hline L DLPFC & 45 & 70 & -42 & 41 & 23 & 3.98 \\
\hline \multicolumn{7}{|c|}{ B) Trained Group $(\mathrm{S} 1>\mathrm{S3})>$ Control Group $(\mathrm{S} 1>\mathrm{S3})$} \\
\hline L DLPFC & 46 & 125 & -33 & 41 & 20 & 4.14 \\
\hline R DLPFC & 9 & 99 & 15 & 56 & 26 & 3.77 \\
\hline
\end{tabular}

FOOTNOTES: Results were $p<0.05$ FWE cluster-corrected using a threshold of $p<0.001$ at the uncorrected voxel level, and a cluster extension of $\mathrm{k}=70$ voxels and $\mathrm{k}=99$ voxels, respectively. $\mathrm{L}=$ Left. $\mathrm{R}=$ Right. BA: Brodmann Area. DLPFC: dorsolateral prefrontal cortex.

\section{Brain-behavior correlation analysis results}

The relationship between performance improvement from S1 to S2 and brain changes after training was studied by means of bivariate Pearson's correlation analysis. MNI coordinates used to create the regions of interest (ROIs) are shown in Table 1. We used the peak maximum of the regions that had decreased activation on the PASAT in S2 and $\mathrm{S} 3$, as described in the preceding paragraphs.

Improvement in the PASAT after training. ROI data were correlated with the improvement on the PASAT accuracy score after training in S2 to find out whether behavioral improvement in the trained group was associated with brain changes. The analysis showed a significant negative correlation between the activation in the left DLPFC lobe in S2 and the improvement on the PASAT task in S2 in the trained group 
$(\mathrm{r} 25=-0.42 \mathrm{p}=.035$; Control group: $\mathrm{r} 25=0.12 \mathrm{p}=.546)$. The difference between these two correlation coefficients was statistically significant (Trained group > Control group, $\mathrm{z}=-1.93 \mathrm{p}=.027$, one-tailed). Similarly, we found a significant negative correlation between the activation in the left DLPFC in S3 and the improvement on the PASAT task in $\mathrm{S} 2$ in the trained group $(\mathrm{r} 25=-0.41 \mathrm{p}=0.044$; Control group: $\mathrm{r} 25=0.006 \mathrm{p}=.979$; see

Figure 4 for scatterplots of the meaningful correlations). These analyses may demonstrate that participants with a greater decrease in DLPFC showed more improvement in their PASAT performance after $n$-back training.

Figure 4: Scatterplots of the correlations between the improvement in PASAT scores in the post-training session and the left DLPFC in the post-training and follow-up sessions. Circular dark dots correspond to the trained group, and squared light dots correspond to the control group. Asterisk $\left(^{*}\right)$ indicates the significant correlations. N=52 (Trained group $=25$; Control group $=27$ ).

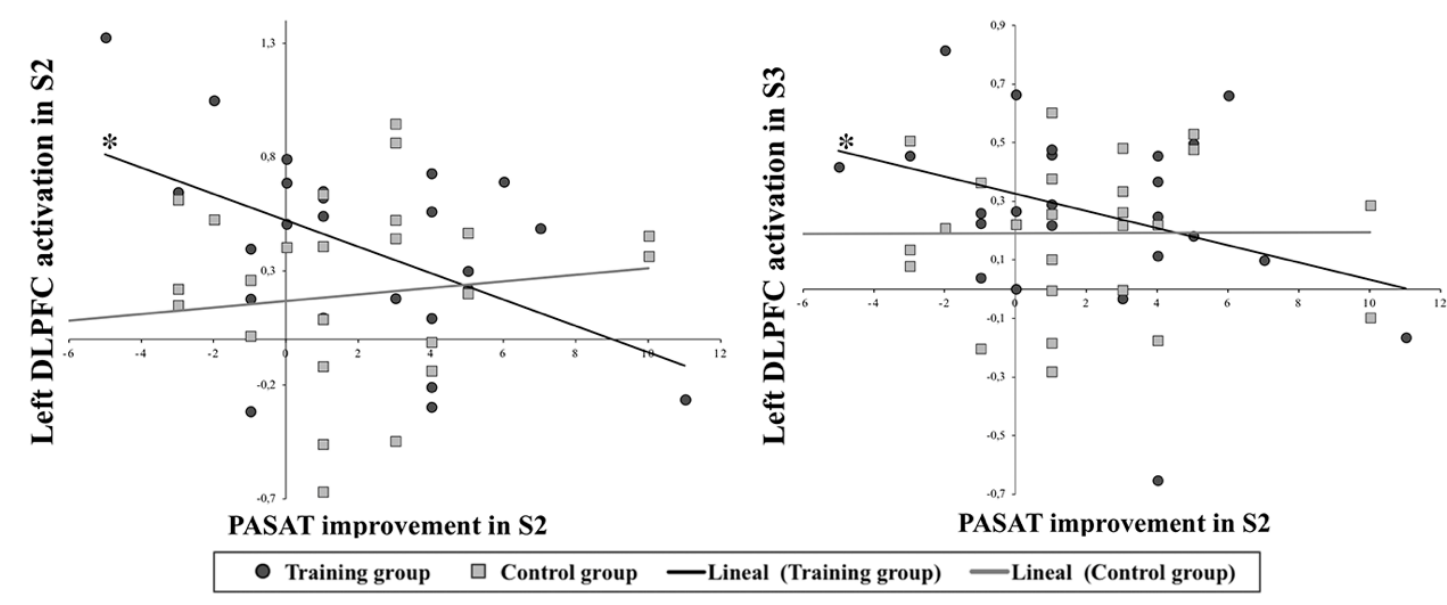

Improvement in $\boldsymbol{n}$-back after training. The ROI data obtained were also correlated with behavioral improvement in the $n$-back processing after training in the trained group. Analyses yielded a significant negative correlation between the right DLPFC in S2 with the improvement in 2-back in TS4 $(\mathrm{r} 25=-0.54 \mathrm{p}=0.005)$. No significant correlations were found associated with 3-back. 


\section{PASAT and n-back activation spatial overlap}

To verify that PASAT and $n$-back involve the same brain areas, a conjunction analysis of the two tasks was conducted using both groups' data collected in S1. Results were $\mathrm{p}<.05$ FWE cluster-corrected using a threshold of $\mathrm{p}<.001$ at the uncorrected voxel level and a cluster extension of $\mathrm{k}=187$. The same participants that completed the present study previously completed an $n$-back fMRI study (Miró-Padilla et al., 2018); therefore, their data were used to conduct the aforementioned analysis. Figure $5 \mathbf{A}$ shows that the same areas were activated during the performance of each task: bilateral superior, middle and inferior frontal cortex (BA 6/8-11/32/45-48), including the supplementary motor area/anterior cingulate gyrus (SMA/ACC) (BA 6/32) and the anterior insula (BA 13), bilateral superior and inferior parietal cortex (BA 7/40), including the precuneus, bilateral inferior temporal cortex (BA 20), bilateral cerebellum (crus I), and two midbrain areas (thalamus and globus pallidus). Additionally, in order to ascertain that our results showing decreased activation during the performance of the PASAT matched the activations on the $n$-back trained task, an overlap between the results of the $n$-back general task activation in the trained group and the interaction analyses (stated in previous paragraphs) in S2 (Figure 5B) and in S3 (Figure 5C) was also included. $N$-back general task activation in the trained group in $\mathrm{S} 1$ was obtained from the data from our previous study (Miró-Padilla et al., 2018), and the 2-back + 3-back>0-back contrast was used to procure the task-activation map for the spatial overlap. Finally, the brain maps were saved and overlaid in MRICron over the template ch2bet.nii.

Figure 5: Regions in which overlapping activation were observed between the training ( $n$-back) and the transfer task (PASAT). The $n$-back data were obtained from our previous study (37) of the same participants who completed the present study. (A) Conjunction analysis between $n$-back (contrast: 2-back + 3back>0-back) and PASAT (contrast: $a d d>$ repeat). The same areas were activated during the performance of both tasks. 
Results were $\mathrm{p}<.05$ FWE cluster-corrected using a threshold of $\mathrm{p}<.001$ at the uncorrected voxel level and a cluster extension of $\mathrm{k}=187$. (B) Overlap of the $n$-back general task activation in the trained group in $\mathrm{S} 1$ with the decreased activation found in the DLPFC during PASAT performance in S2 (the same as the Figure 3A). We used MRICron software to overlap the results and create the figure. Therefore, the yellow scale represents the contrast: 2-back +3 back $>0$-back, and the blue scale represents the contrast: Trained group $(\mathrm{S} 1>\mathrm{S} 2)>$ Control group $(\mathrm{S} 1>\mathrm{S} 2)$. Results were $\mathrm{p}<.05 \mathrm{FWE}$ cluster-corrected using a threshold of $\mathrm{p}<.005$ at the uncorrected voxel level and a cluster extension of $\mathrm{k}=1500$ voxels for $n$-back and a threshold of $\mathrm{p}<.001$ at the uncorrected voxel level and a cluster extension of $\mathrm{k}=70$ voxels for PASAT. (C) Overlap of the $n$-back general task activation in the trained group in $\mathrm{S} 1$ with the decreased activation found in the DLPFC during PASAT performance in S3 (the same as the Figure 3B). Therefore, the yellow scale represents the contrast: 2-back + 3back>0-back, and the blue scale represents the contrast: Trained group $(\mathrm{S} 1>\mathrm{S} 3)>$ Control group $(\mathrm{S} 1>\mathrm{S} 3)$. Results were $\mathrm{p}<.05$ FWE cluster-corrected using a threshold of $\mathrm{p}<.005$ at the uncorrected voxel level and a cluster extension of $\mathrm{k}=1500$ voxels for $n$-back and, for PASAT, a threshold of $\mathrm{p}<.001$ at the uncorrected voxel level and a cluster extension of $\mathrm{k}=99$ voxels.

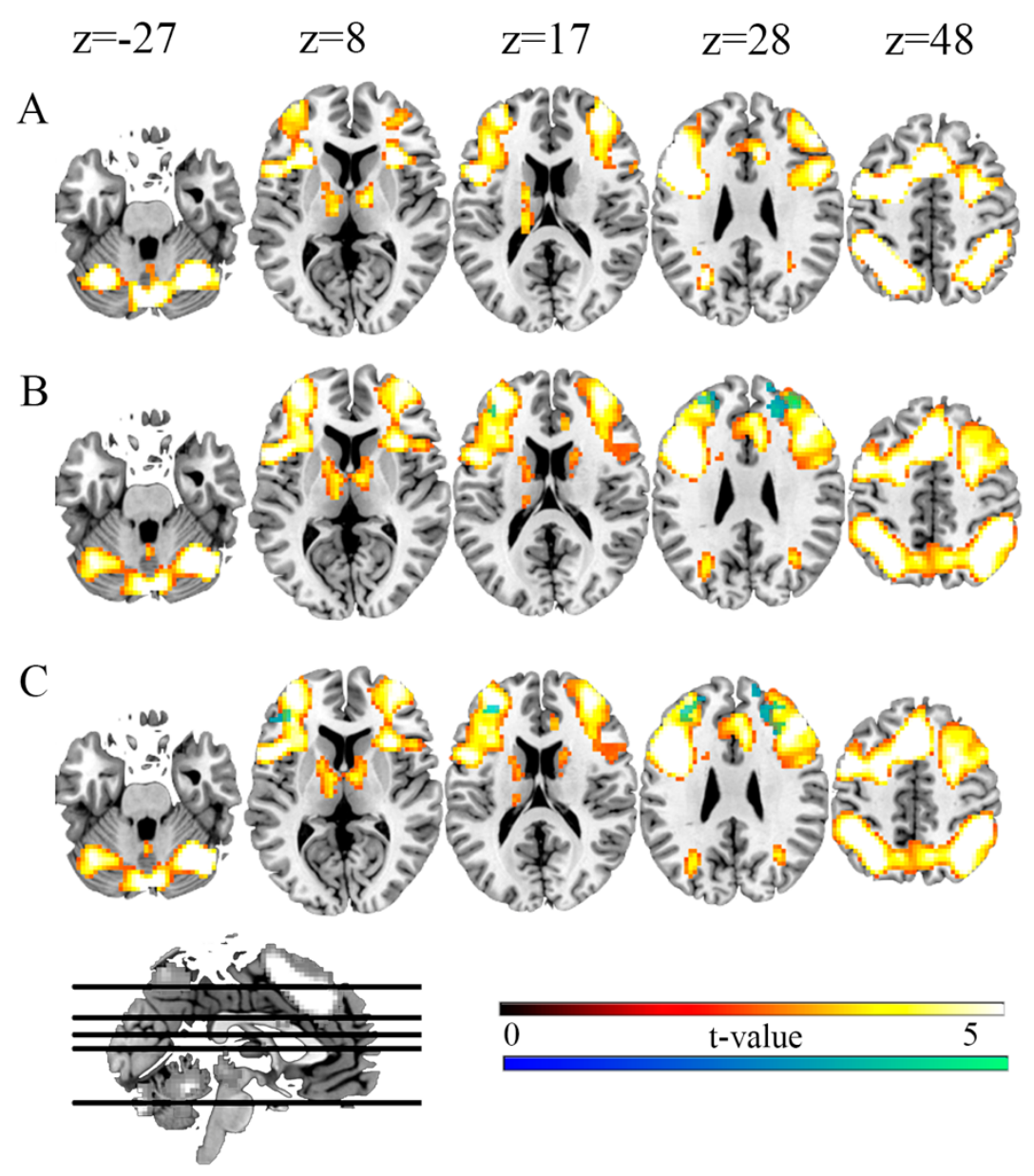




\section{DISCUSSION}

In the current study, the behavioral and neural far transfer effects from a working memory training task (n-back) to another untrained working memory task (PASAT) were examined. Previous literature has found mixed evidence supporting transfer effects, and whether or not the tasks share the same brain systems seems to be a determinant in observing these effects. In the present study, we tested whether two robust but clearly different working memory tasks support the far transfer effect phenomenon. Although the behavioral analysis results show no global transfer effects, the brain imaging analysis results indicate that neural transfer effects have occurred. These effects consisted of a decrease in activation in the bilateral anterior DLPFC, and they were found immediately after training and five weeks later. In addition, these brain changes were linked to behavioral improvement. These results demonstrate that a working memory training program improves the neural processing of an untrained task that shares the use of the central executive, suggesting some kind of improved neural efficiency.

Our results showed that both the control and trained groups improved their performance after training. The use of an easy version of the transfer task (i.e. 3 second inter-stimulus interval) allowed us to minimize the possible bias of performance differences on brain activations; that is, we obtained a homogenous performance across subjects. The mean accuracy at baseline was high, but it allowed significant and small improvements (i.e. $3 \%$ ) in post-training sessions. This mean improvement was equal for both groups and may be mainly due to the retest effect because repeated exposure to the PASAT has previously demonstrated significant increases in performance (Tombaugh, 2006). This means that only some of the participants in the trained group behaviorally showed a significant transfer effect from $n$-back training, whereas the performance of the others did 
not improve due to training or retest. In this regard, our experiment adds to others that have failed to observe behavioral far transfer after working memory training (Chooi \& Thompson, 2012; Clark et al., 2017a; 2017b; Harrison et al., 2013; Minear et al., 2016; Redick \& Lindsey, 2013; Redick et al., 2013; Salminen et al., 2012; Thompson et al., 2013; Waris et al., 2015).

In relation to brain activity, PASAT task activations mainly include frontal and parietal areas related to working memory. This pattern coincides with previous neuroimaging studies that used the PASAT (Audoin et al., 2003; Forn et al., 2011), and it strongly overlaps with the $n$-back task activation pattern, as shown in Figure 5A. The overlap with the $n$-back affects the bilateral DLPFC, SMA/ACC, insula, bilateral parietal cortex, bilateral inferior temporal cortex, bilateral cerebellum, and two midbrain areas (thalamus and globus pallidus). Both the PASAT and $n$-back are working memory tasks where participants have to maintain verbal information for a short period of time and give a response (Jaeggi et al., 2010a; Tombaugh, 2006). However, the tasks differ substantially in the type of stimuli, the kind of cognitive manipulations (on the PASAT they have to add simple numbers, and on the $n$-back they have to retain a different number of letters) and the type of response (verbal or manual), preventing similar processes controlled by posterior areas of the brain. Thus, the general neural overlap represents the common use of the working memory network, but the cognitive processes involved in each task only have in common the need to maintain verbal information, a process that is more related to the central executive component of working memory.

As far as we know, this is the first study to show the neural transfer effects located in crucial areas involved in working memory, such as the anterior prefrontal cortex. The 
anterior DLPFC cortex acts as the central executive in working memory, with a flexible role that operates at the abstract level, modulating the processing of posterior brain structures (Constantinidis \& Klingberg, 2016; D’Esposito \& Postle, 2015). Importantly, the area obtained overlapped with both the 2-back and 3-back and the PASAT, indicating that this area participates in both tasks. Considering the cognitive processes involved in both tasks, the maintenance of verbal information for seconds is the only process they have in common, and this process is controlled from the anterior DLPFC. Thus, we have verified the hypothesis that transfer effects occur if the training task and the transfer task involve the same brain regions and cognitive processes.

There is an intense debate about how to interpret this decrease in neural activation after training (Constantinidis \& Klingberg, 2016; Poldrack, 2015). These recent views have a critical vision of the neural efficiency explanation, where participants seemed not to need so many brain resources and effort to produce less mistakes, classifying it like simply and unclear. The review by Constantinidis and Klingberg (2016) compared the effects of working memory training using neurophysiological recordings in non-human primates and neuroimaging data in humans. Whereas data on primates shows that training increases the activity of prefrontal neurons and the strength of connectivity within the prefrontal cortex, human data shows a decreased activation on the trained task after training, which was typically interpreted as indicating increased neural efficiency (Buschkuehl et al., 2014; Kelly, Foxe, \& Garavan, 2006). When analyzing all the literature, in primate studies increases were found in the ventrolateral, but not the DLPFC (Constantinidis \& Klingberg, 2016), whereas results in humans were found in dorsolateral areas. Thus, we can interpret present results as increased neural efficiency. Moreover, in the trained group, our results also showed a correlation between the decrease in activation 
on the DLPFC and the improvement on the PASAT task after training. However, no relationship was found for the control group. This result is consistent with the neural efficiency interpretation because the participants in the trained group with a greater decrease were also the ones with better performance. Because the DLPFC is involved in top-down control (Edin et al., 2009), the decrease may reflect a lower cognitive control requirement after training to attain a correct response.

Importantly, the decreases observed after training persisted with no change after five weeks without training. Previous research found stability effects on the trained task $(n-$ back; Chang et al., 2017; Miró-Padilla et al., 2018), but this is the first time the stability of neural transfer effects has been demonstrated. A limitation of our study was the use of a no-contact control group instead of an active control group, which might result in motivational differences between the two groups in terms of task efficiency. The observed gains in the trained group may not be due to working memory training per se, but rather to training in general. Soveri et al.(2017), in their meta-analysis, did not find significantly different training effects depending on the type of control group. Moreover, the fact that neural changes remain after five weeks of no training increases the possibility of a general effect of training.

Our results differ significantly from studies reporting neural near transfer in the striatum (Dahlin et al., 2008; Salminen et al., 2016). These studies used two similar tasks requiring the person to learn certain cognitive processes, such as letter updating (Dahlin et al., 2008) or dual-task working memory (Salminen et al., 2016), which are transferred from the trained to the transfer task. The striatum is involved in these specific transfers that require the regulation of the information that is relevant to the working memory task. This process 
is not involved in the far transfer in our experiment. In fact, unless we used an easy version of the task, the PASAT is a very demanding task with a clear component of cognitive control (involving maintenance of stimuli and, probably, inhibition of responses), and this is the process that is probably transferred from the $n$-back training.

In conclusion, our results showed near transfer neural effects associated with the improvement on the PASAT task. $N$-back training caused a decrease in the activation of the DLPFC during PASAT performance, which indicates a clear neural transfer effect. These cerebral changes remained stable after five weeks and were related to improvement on the task, as revealed by the correlations results. These results point in the direction of the hypothesis that transfer will occur if the training and the transfer task involve the same brain regions and processing components. Future research should focus on how this kind of working memory regime affects a clinical population, and test whether the stability of these effects lasts longer than five weeks.

\section{REFERENCES}

Au, J., Buschkuehl, M., Duncan, G. J., \& Jaeggi, S. M. (2015). There is no convincing evidence that working memory training is NOT effective: A reply to Melby-Lervåg and Hulme (2015). Psychonomic Bulletin \& Review, 3(1), 331-337. https://doi.org/10.3758/s13423-015-0967-4

Au, J., Sheehan, E., Tsai, N., Duncan, G. J., Buschkuehl, M., \& Jaeggi, S. M. (2015). Improving fluid intelligence with training on working memory: a meta-analysis. Psychonomic Bulletin \& Review, 22(2), 366-377. https://doi.org/10.3758/s13423014-0699-X

Audoin, B., Ibarrola, D., Ranjeva, J. P., Confort-Gouny, S., Malikova, I., Ali-Chérif, A., ... Cozzone, P. (2003). Compensatory cortical activation observed by fMRI during a cognitive task at the earliest stage of MS. Human Brain Mapping, 20(2), 51-58. https://doi.org/10.1002/hbm.10128

Buschkuehl, M., Hernandez-Garcia, L., Jaeggi, S. M., Bernard, J. a, \& Jonides, J. (2014). Neural effects of short-term training on working memory. Cognitive, Affective \& Behavioral Neuroscience, 14(1), 147-160. https://doi.org/10.3758/s13415-0130244-9

Buschkuehl, M., Jaeggi, S. M., \& Jonides, J. (2012). Neuronal effects following working 
memory training. Developmental Cognitive Neuroscience, 2, Supplem, S167-S179. https://doi.org/10.1016/j.den.2011.10.001

Chang, L., Løhaugen, G. C., Andres, T., Jiang, C. S., Douet, V., Tanizaki, N., ... Ernst, T. M. (2017). Adaptive working memory training improved brain function in human immunodeficiency virus-seropositive patients. Annals of Neurology, 81(1), 17-34. https://doi.org/10.1002/ana.24805

Chooi, W.-T., \& Thompson, L. A. (2012). Working memory training does not improve intelligence in healthy young adults. Intelligence, 40(6), 531-542. https://doi.org/10.1016/J.INTELL.2012.07.004

Clark, C. M., Lawlor-Savage, L., \& Goghari, V. M. (2017a). Working memory training in healthy young adults: Support for the null from a randomized comparison to active and passive control groups, 1-25. https://doi.org/10.6084/m9.figshare.4551517

Clark, C. M., Lawlor-Savage, L., \& Goghari, V. M. (2017b). Functional Brain Activation Associated with Working Memory Training and Transfer. Behavioural Brain Research, 334(May), 34-49. https://doi.org/10.1016/j.bbr.2017.07.030

Constantinidis, C., \& Klingberg, T. (2016). The neuroscience of working memory capacity and training. Nature Reviews Neuroscience, 17(7), 438-449. https://doi.org/10.1038/nrn.2016.43

Correia, S. (2011). Paced Auditory Serial Attention Test. In J. S. Kreutzer, J. DeLuca, \& B. Caplan (Eds.), Encyclopedia of Clinical Neuropsychology (pp. 1840-1845). New York, NY: Springer New York. https://doi.org/10.1007/978-0-387-79948-3_1319

D'Esposito, M., \& Postle, B. R. (2015). The Cognitive Neuroscience of Working Memory. Annu Rev Psychol., (66), 115-142. https://doi.org/10.1146/annurevpsych-010814-015031.THE

Dahlin, E., Neely, A. S., Larsson, A., Bäckman, L., \& Nyberg, L. (2008). Transfer of learning after updating training mediated by the striatum. Science (New York, N.Y.), 320(5882), 1510-1512. https://doi.org/10.1126/science.1155466

Duncan, J., \& Owen, A. M. (2000). Common regions of the human frontal lobe recruted by diverse cognitive demands. Trends in Neurosciences, 23(10), 475-483. https://doi.org/10.1016/S0166-2236(00)01633-7

Edin, F., Klingberg, T., Johansson, P., McNab, F., Tegnér, J., \& Compte, A. (2009). Mechanism for top-down control of working memory capacity. Proceedings of the National Academy of Sciences of the United States of America, 106(16), 68026807. https://doi.org/10.1073/pnas.0901894106

Forn, C., Barros-Loscertales, A., Escudero, J., Belloch, V., Campos, S., Parcet, M. A., \& ??vila, C. (2006). Cortical reorganization during PASAT task in MS patients with preserved working memory functions. NeuroImage, 31(2), 686-691. https://doi.org/10.1016/j.neuroimage.2005.12.030

Forn, C., Belenguer, A., Belloch, V., Sanjuan, A., Parcet, M. A., \& Avila, C. (2011). Anatomical and functional differences between the Paced Auditory Serial Addition Test and the Symbol Digit Modalities Test. Journal of Clinical and Experimental Neuropsychology, 33(1), 42-50. https://doi.org/10.1080/13803395.2010.481620

Friston, K. J., Holmes, A. P., Worsley, K. J., Poline, J.-P., Frith, C. D., \& Frackowiak, R. S. J. (1995). Statistical Parametric Maps in Functional Imaging: A General Linear Approach. Human Brain Mapping, 2(4), 189-210. https://doi.org/10.1002/hbm.460020402

Harrison, T. L., Shipstead, Z., Hicks, K. L., Hambrick, D. Z., Redick, T. S., \& Engle, R. W. (2013). Working Memory Training May Increase Working Memory Capacity but Not Fluid Intelligence. Psychological Science, 24(12), 2409-2419. 
https://doi.org/10.1177/0956797613492984

Jaeggi, S. M., Buschkuehl, M., Jonides, J., \& Perrig, W. J. (2008). Improving fluid intelligence with training on working memory. Proceedings of the National Academy of Sciences of the United States of America, 105(19), 6829-6833. https://doi.org/10.1073/pnas.0801268105

Jaeggi, S. M., Buschkuehl, M., Jonides, J., \& Shah, P. (2011). Short- and long-term benefits of cognitive training. Proceedings of the National Academy of Sciences, 108(25), 10081-10086. https://doi.org/10.1073/pnas.1103228108

Jaeggi, S. M., Buschkuehl, M., Perrig, W. J., \& Meier, B. (2010a). The concurrent validity of the N-back task as a working memory measure. Memory, 18(4), 394-412. https://doi.org/10.1080/09658211003702171

Jaeggi, S. M., Studer-Luethi, B., Buschkuehl, M., Su, Y.-F., Jonides, J., \& Perrig, W. J. (2010b). The relationship between n-back performance and matrix reasoning implications for training and transfer. Intelligence, 38(6), 625-635. https://doi.org/10.1016/j.intell.2010.09.001

Karbach, J., \& Kray, J. (2009). How useful is executive control training? Age differences in near and far transfer of task-switching training. Developmental Science, 12(6), 978-990. https://doi.org/10.1111/j.1467-7687.2009.00846.x

Karbach, J., \& Verhaeghen, P. (2014). Making Working Memory Work: A MetaAnalysis of Executive-Control and Working Memory Training in Older Adults. Psychological Science, 25(11), 2027-2037. https://doi.org/10.1177/0956797614548725

Kelly, C., Foxe, J. J., \& Garavan, H. (2006). Patterns of Normal Human Brain Plasticity After Practice and Their Implications for Neurorehabilitation. Archives of Physical Medicine and Rehabilitation, 87(12 SUPPL.), 20-29. https://doi.org/10.1016/j.apmr.2006.08.333

Klingberg, T. (2010). Training and plasticity of working memory. Trends in Cognitive Sciences, 14(7), 317-324. https://doi.org/10.1016/j.tics.2010.05.002

Küper, K., \& Karbach, J. (2016). Increased training complexity reduces the effectiveness of brief working memory training: evidence from short-term single and dual $\mathrm{n}$-back training interventions. Journal of Cognitive Psychology, 28(2), 199-208. https://doi.org/10.1080/20445911.2015.1118106

Melby-Lervåg, M., \& Hulme, C. (2016). There is no convincing evidence that working memory training is effective: A reply to Au et al. (2014) and Karbach and Verhaeghen (2014). Psychonomic Bulletin \& Review, 23(1), 324-330. https://doi.org/10.3758/s13423-015-0862-z

Melby-Lervåg, M., Redick, T., \& Hulme, C. (2016). Working Memory Training Does Not Improve Performance on Measures of Intelligence or Other Measures of "Far Transfer": Evidence from a Meta-Analytic Review. Perspectives on Psychological Science, 11(4). https://doi.org/10.3837/tiis.0000.00.000

Minear, M., Brasher, F., Guerrero, C. B., Brasher, M., Moore, A., \& Sukeena, J. (2016). A simultaneous examination of two forms of working memory training: Evidence for near transfer only. Memory \& Cognition, 1-24. https://doi.org/10.3758/s13421016-0616-9

Miró-Padilla, A., Bueichekú, E., Ventura-Campos, N., Flores-Compañ, M.-J., Parcet, M. A., \& Ávila, C. (2018). Long-term brain effects of N-back training: an fMRI study. Brain Imaging and Behavior. https://doi.org/10.1007/s11682-018-9925-X

Morrison, A. B., \& Chein, J. M. (2011). Does working memory training work? The promise and challenges of enhancing cognition by training working memory. Psychonomic Bulletin \& Review, 18(1), 46-60. https://doi.org/10.3758/s13423- 
010-0034-0

Owen, A. M., McMillan, K. M., Laird, A. R., \& Bullmore, E. (2005). N-back working memory paradigm: a meta-analysis of normative functional neuroimaging studies. Human Brain Mapping, 25(1), 46-59. https://doi.org/10.1002/hbm.20131

Poldrack, R. A. (2015). Developmental Cognitive Neuroscience Is " efficiency " a useful concept in cognitive neuroscience ? Developmental Cognitive Neuroscience, 11, 12-17. https://doi.org/10.1016/j.dcn.2014.06.001

Redick, T. S., \& Lindsey, D. R. B. (2013). Complex span and n-back measures of working memory: A meta-analysis. Psychonomic Bulletin \& Review, 20(6), 1102-1113. https://doi.org/10.3758/s13423-013-0453-9

Redick, T. S., Shipstead, Z., Harrison, T. L., Hicks, K. L., Fried, D. E., Hambrick, D. Z., ... Engle, R. W. (2013). No Evidence of Intelligence Improvement After Working Memory Training: A Randomized, Placebo-Controlled Study. Journal of Experimental Psychology: General, 142(2), 359-379. https://doi.org/10.1037/a0029082

Salminen, T., Kühn, S., Frensch, P. A., \& Schubert, T. (2016). Transfer after Dual n-Back Training Depends on Striatal Activation Change. The Journal of Neuroscience, 36(39), $10198 . \quad$ Retrieved from http://www.jneurosci.org/content/36/39/10198.abstract

Salminen, T., Strobach, T., \& Schubert, T. (2012). On the impacts of working memory training on executive functioning. Frontiers in Human Neuroscience, 6(June), 1-14. https://doi.org/10.3389/fnhum.2012.00166

Schweizer, S., Grahn, J., Hampshire, A., Mobbs, D., \& Dalgleish, T. (2013). Training the emotional brain: Improving affective control through emotional working memory training. Annals of Internal Medicine, 158(6), 5301-5311. https://doi.org/10.1523/JNEUROSCI.2593-12.2013

Soveri, A., Antfolk, J., Karlsson, L., Salo, B., \& Laine, M. (2017). Working memory training revisited: A multi-level meta-analysis of n-back training studies. Psychonomic Bulletin \& Review. https://doi.org/10.3758/s13423-016-1217-0

Thompson, T. W., Waskom, M. L., Garel, K. L. A., Cardenas-Iniguez, C., Reynolds, G. O., Winter, R., ... Gabrieli, J. D. E. (2013). Failure of Working Memory Training to Enhance Cognition or Intelligence. PLoS ONE, 8(5). https://doi.org/10.1371/journal.pone.0063614

Tombaugh, T. N. (2006). A comprehensive review of the Paced Auditory Serial Addition Test ( PASAT ), 21, 53-76. https://doi.org/10.1016/j.acn.2005.07.006

von Bastian, C. C., \& Oberauer, K. (2014). Effects and mechanisms of working memory training: a review. Psychological Research, 78(6), 803-820. https://doi.org/10.1007/s00426-013-0524-6

Waris, O., Soveri, A., \& Laine, M. (2015). Transfer after Working Memory Updating Training, 1-19. https://doi.org/10.1371/journal.pone.0138734

Woo, C.-W., Krishnan, A., \& Wager, T. D. (2014). Cluster-extent based thresholding in fMRI analyses: pitfalls and recommendations. NeuroImage, 91, 412-419. https://doi.org/10.1016/j.neuroimage.2013.12.058 
Study 3

Sustained and transient gray matter volume changes after N-back training: a VBM study

Anna Miró-Padilla, Elisenda Bueichekú and César Ávila

Under review 


\begin{abstract}
Working memory training causes cerebral changes in terms of activation and functional connectivity. Nevertheless, the scarce studies that have investigated gray matter (GM) changes after working memory training have produced heterogeneous results and have never investigated the stability effects. The present study was designed to test for sustained and transient anatomic changes after only 200 minutes of working memory training. By means of a longitudinal voxel-based morphometry study, we investigated the GM changes produced by a brief single $n$-back training, immediately and 5 weeks after finishing it, in a sample of 52 participants who were assigned to either a training or a passive control group. Results showed sustained GM volume enlargement in the right superior parietal cortex, and a transient decrement in the right putamen. This last change was stronger in individuals with lower IQ scores. The results provide further evidence that brief working memory training causes plasticity in brain structures related to the trained task.
\end{abstract}

\title{
KEYWORDS
}

Voxel-based morphometry; CAT12; working memory training; $n$-back; gray matter changes 


\section{Introduction}

For a number of years now, research on the effects of working memory training has increased considerably. Numerous behavioral studies have demonstrated that working memory training leads to improvements on the trained tasks (Klingberg, 2010). Neuroimaging investigations, less extended, have provided evidence of different cerebral modifications after working memory training. Activation changes in working memory brain areas have been reported, as well as an enhancement of functional the connectivity between them during the task and even in a resting-state (Constantinidis \& Klingberg, 2016). However, less is known about local anatomic changes after training. The present manuscript aims to investigate how this training affects the structure of the brain in these areas.

As has been well-established, the $n$-back is the most widely used task in neuroimaging studies of working memory training. Both the single and dual versions of the task have been used in training designs, and both have proven their efficacy (Jaeggi, Buschkuehl, Perrig, \& Meier, 2010; Küper \& Karbach, 2016; Soveri, Antfolk, Karlsson, Salo, \& Laine, 2017). In short periods of training, single $n$-back was more effective than dual (Küper \& Karbach, 2016). After working memory training, the activation changes were usually present in brain areas that were already activated during the working memory task before training (Constantinidis \& Klingberg, 2016). The association areas of the prefrontal and the parietal cortex are the most consistently involved areas after training, in most cases showing a reduced contribution to task performance after training (see Buschkuehl, Jaeggi, \& Jonides, 2012, for a review). Literature also suggest that working memory training increases functional connectivity between the prefrontal and parietal cortex, improving the capacity of a stimulus-encoding network (Durstewitz, Seamans, \& 
Sejnowski, 2000; Edin et al., 2009). All this evidence suggests that intense working memory practice increases the integrity and efficiency of frontoparietal networks. A second focus of interest is the striatum and the dopaminergic system. Several studies have described increases in the activation of the striatum linked to working memory training (Dahlin, Neely, Larsson, Bäckman, \& Nyberg, 2008; McNab \& Klingberg, 2007; Salminen, Kühn, Frensch, \& Schubert, 2016). In addition, studies using PET have demonstrated changes in the dopaminergic system as a result of this training (Backman et al., 2011; McNab et al., 2009). The role of the striatum does not seem to be directly related to working memory functions, but it could serve to facilitate plasticity in frontoparietal networks (Constantinidis \& Klingberg, 2016). This role of the striatum is especially developed during the first week of training (Kühn et al., 2013).

If there are activation and functional connectivity changes after training, it seems reasonable to hypothesize that they may be accompanied by structural changes in the same brain areas. Literature has demonstrated training-related anatomic changes in different domains. Specifically, increased gray matter (GM) volume in diverse cerebral areas has been observed after training in physical skills (i.e. juggling) (Draganski et al., 2004), language (Golestani \& Pallier, 2007; Golestani, Paus, \& Zatorre, 2002; Golestani \& Zatorre, 2004; Ilg et al., 2008; Mårtensson et al., 2012; Stein et al., 2012), memory (Draganski et al., 2006; Engvig et al., 2010), or music skills (Bengtsson et al., 2005; Gaser \& Schlaug, 2003a \& 2003b; Hyde et al., 2009; Palomar-García, Zatorre, VenturaCampos, Bueichekú, \& Ávila, 2017; Sluming et al., 2002).

Regarding structural changes specifically after working memory training, research has been scarce. A review reported that working memory capacity positively correlated with 
regional GM and with white matter volume in frontal and parietal regions in different clinical populations (Takeuchi, Taki, \& Kawashima, 2010). Longitudinal studies that employed morphometry and cortical thickness measures have not obtained a common pattern of results (Colom et al., 2016; Metzler-Baddeley, Caeyenberghs, Foley, \& Jones, 2016; Takeuchi et al., 2011). In some cases, they reported changes in brain areas not related to working memory. The different procedures and methodological strategies and the lack of correlation between changes and performance measures may explain these differences. Beyond them, a general gap in longitudinal training studies involves the need to determine the stability of structural changes over time. The interpretation of results of neuroanatomical changes after training would benefit from determining if the changes are transient or stable, in order to establish their functional significance.

By means of a longitudinal VBM study, this study analyzed the brains of a sample of healthy adults before training, after $200 \mathrm{~min}$ on an adaptive version of single $n$-back training, and five weeks after finishing the training. Our previous study showed that this training improved $n$-back performance in terms of accuracy and response speed until 5 weeks after training, and it was associated with a decrease in the activation in frontoparietal areas (Miró-Padilla et al., 2018). Obtaining MRI structural data at 3 time points would allow us to investigate sustained and transient GM volume changes. We hypothesized that sustained changes would be present in frontoparietal areas as a result of the continuous use of these cortical areas during training and the maintenance of behavioral improvements. Moreover, transient changes after training would be observed in the striatum due to its role in facilitating cortical plasticity without any participation in retraining of the working memory processes. 


\section{Materials and methods}

\subsection{Participants}

In the present study, 52 healthy right-handed participants (20 male) participated. All were recruited from the student population of the Universitat Jaume I, and none of them reported a previous psychiatric or neurologic diagnosis. The Ethical Committee of the Universitat Jaume I approved the research project, and informed consent was obtained from each subject before participation. For their active participation, they received monetary compensation. Participants were randomly assigned to either an experimental condition (trained group) $(\mathrm{N}=25$, mean age $=22.72 \pm 1.51,10$ men $)$ or a control condition (control group) $(\mathrm{N}=27$, mean age $=22.52 \pm 1.45,10$ men). Their intellectual level was assessed with the Matrix Reasoning Test (WAIS-III-R) (trained group: mean=21.08 \pm 3.35 ; control group: mean=21.63 \pm 1.94 ). Between-group differences in gender distribution, age, and IQ were non-significant.

\subsection{Experimental Design}

Both groups completed three fMRI sessions with the same adapted block-design $n$-back task. The second fMRI session took place 7 days after the first one, and the last one 35 more days (five weeks) after the second (Figure 1). The trained group trained 200 minutes on an adaptive $n$-back task between the two first scanner sessions. The groups did not have any training between the two last sessions. The pre-training session, posttraining session, and follow-up session correspond to Session 1 (S1), Session 2 (S2), and Session 3 (S3), respectively. Visual stimuli (letters) were presented electronically using E-Prime software (Psychology Software Tools, Pittsburgh, PA), professional version 2.0, installed in a Hewlett-Packard portable workstation (screen-resolution 800 x 600, refresh rate of $60 \mathrm{~Hz}$ ). Participants watched the laptop screen through MRI-compatible goggles 
(VisuaStim, Resonance Technology, Inc., Northridge, CA, USA), and their responses were collected via MRI-compatible response-grips (NordicNeuroLab, Bergen, Norway).

Figure 1: Schematic description of the experimental design. Both groups performed the same $n$-back task in the three fMRI sessions. Training group data correspond to the green circles and control group data to the blue squares. Training consisted of an adaptive single $n$-back task, 4 sessions, 200 minutes.

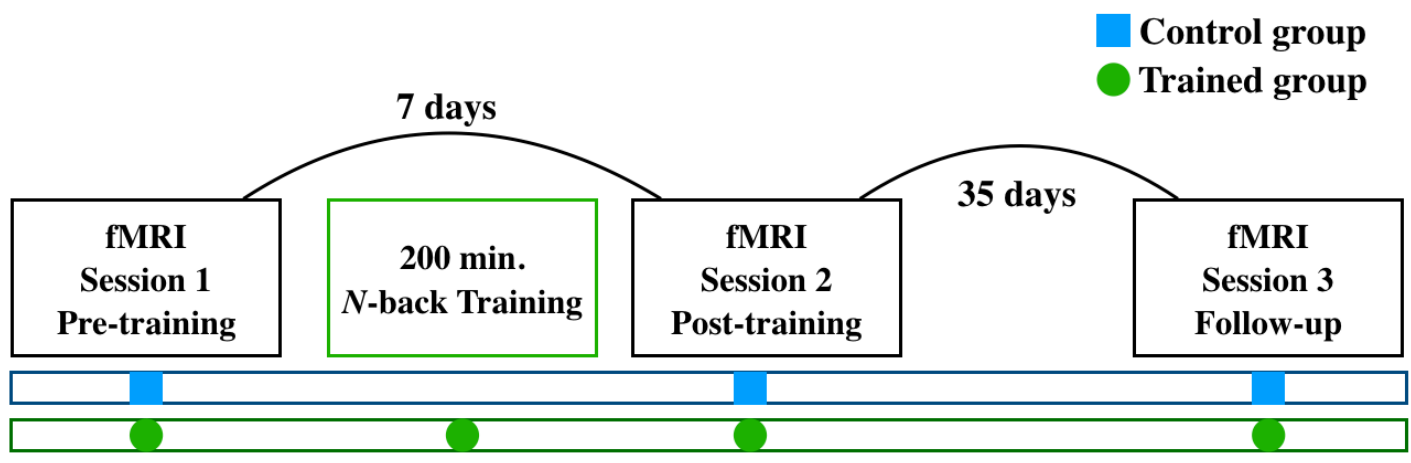

\section{3. $N$-back fMRI task}

As described in our previous study (Miró-Padilla et al., 2018), the task was presented in three load levels: two working memory blocks (2-back and 3-back) and a baseline control task (0-back). In 0-back, subjects pressed the "yes" button when the target (letter X) appeared on the screen, and they responded "no" to any other letters. In the 2-back and 3-back load levels, participants pressed the "yes" button when the current letter shown on the screen matched the one presented 2 or 3 items back, and they pressed "no" when there were no targets. Subjects had to give manual responses with only their right hand, responding to targets with their thumb and to non-targets with their forefinger. The EPrime's logfile saved each participant's accuracy and reaction time (RT) to each stimulus. 


\section{4. $N$-back training task}

As described in our previous study (Miró-Padilla et al., 2018), the trained group carried out four consecutive training sessions (TS) of single $n$-back after fMRI S1 in our laboratory located at the University. Participants performed only one TS per day. One TS lasted 60 minutes and was distributed in two phases: the learning part and the test part. In the learning part, participants performed an adaptive $n$-back paradigm adapted from Jaeggi et al. (2008) for 50 minutes, whereas in the test part, they performed a simple $n$ back task, which lasted 10 minutes. Therefore, the total training time was approximately 200 minutes, plus 40 minutes for the test part. We used the same laptop as in the fMRI sessions, with the same display features and the same hardware for manual responses.

\subsection{Neuroimaging data acquisition}

Functional MRI data were collected on a 1.5T Siemens Symphony scanner (Erlangen, Germany). The same sequences were used in the three sessions. First, a high-resolution structural T1-weighted MPRAGE sequence was acquired $(\mathrm{TR}=2200 \mathrm{~ms}$; TE $=3 \mathrm{~ms}$; flip angle $90^{\circ}$, matrix $=256 \times 256 \times 160$; voxel size $\left.=1 \times 1 \times 1 \mathrm{~mm}\right)$. Finally, for task-fMRI, a gradient-echo $\mathrm{T} 2 *$-weighted echo-planar MR sequence covering the entire brain was used $\left(\mathrm{TR} / \mathrm{TE}=2500 / 49 \mathrm{~ms}\right.$, matrix $=64 \times 64 \times 28$, flip angle $=90^{\circ}$, voxel size $=3.5 \times 3.5 \times 4.48$; slice thickness $=4 \mathrm{~mm}$; slice gap $=0.48 \mathrm{~mm}$ ). A total of 260 volumes were recorded for $n$-back. All the scanner acquisitions were performed in parallel to the anterior commissure-posterior commissure plane (AC-PC), and they covered the entire brain. Participants were placed in a supine position in the MRI scanner, and their heads were immobilized with cushions to reduce motion degradation. Furthermore, participants were asked to minimize head movement even while giving the answers. 


\subsection{Neuroimaging analysis}

\subsubsection{Voxel-Based Morphometry Preprocessing}

Voxel-based morphometry (VBM) was performed with the Computational Anatomy Toolbox (CAT12; http://dbm.neuro.uni-jena.de/cat/) for the SPM12 package (Wellcome Department of Imaging Neuroscience, London, England; www.fil.ion.ucl.ac.uk/spm/) in the MatLab environment (www.mathworks.com). First of all, each subject's anatomical image was reoriented to the AC-PC plane to have the same point of origin (anterior commissure). Then, the standard preprocessing procedure using Segment Longitudinal Data module in the CAT12 manual was performed. The analysis of the longitudinal data requires customized processing that takes into account the characteristics of the intrasubject analysis. The preprocessing included: 1) registration to the mean image for each subject by an inverse-consistent realignment; 2) registration to a standard template provided by the International Consortium of Brain Mapping (ICBM); 3) segmentation of the images into GM, white matter, and cerebrospinal fluid; 4) DARTEL normalization of the GM segments to the MNI template; and 5) modulation by nonlinear components derived from spatial normalization. After the preprocessing, the total intracranial volume (TIV) was estimated for each subject in each session, and a data quality check was carried out using a CAT12 toolbox to assess the homogeneity of the GM tissues. Finally, images were spatially smoothed using an 8-mm FWHM Gaussian kernel.

\subsubsection{Voxel-Based Morphometry Post-processing Analysis}

For the VBM analysis, we used the CAT12 for all the statistical analyses of interest within the framework of the general linear model. For all analyses, age, sex, and TIV were included as covariates. The GM volume of the control group and the trained group was compared across the sessions. The statistical significance threshold for the whole-brain 
analysis was set at $\mathrm{p}<.05 \mathrm{FWE}$ corrected at the cluster level, with a voxel-level primary threshold of $\mathrm{p}<.001$, uncorrected. First, $\mathrm{S} 1$ data were used to perform a two-sample $t$ test to examine the equality of the GM volume in the two groups, so that between-group brain differences found in subsequent sessions would not be due to preexisting differences. A longitudinal analysis was performed, with interaction analysis between sessions to evaluate: 1) Sustained changes: we investigated sustained effects of training, that is, stable changes due to training by comparing S1 to the average between S2 and S3 (Trained group $(\mathrm{S} 1<\mathrm{S} 2, \mathrm{~S} 3)$ vs. Control group $(\mathrm{S} 1<\mathrm{S} 2, \mathrm{~S} 3))$; and 2) Transient changes: we investigated changes due to training that were recovered in the long-term using a polynomic contrast (Trained group $(\mathrm{S} 1, \mathrm{~S} 3<\mathrm{S} 2)$ vs. Control group $(\mathrm{S} 1, \mathrm{~S} 3<\mathrm{S} 2)$ ).

In addition, the association between the IQ level and the changes in GM volume was tested by calculating the GM volume (in $\mathrm{ml}$ ) of each participant from the clusters of the regions with significant GM volume changes after the training. The clusters were created with the SPM from the MNI coordinates of the local maxima of each contrast. Then, the modulated GM volumes (without smooth) were obtained for each structure via a MATLAB script (http://www0.cs.ucl.ac.uk/staff/g.ridgway/vbm/get_totals.m). Next, to study the brain-behavior relationships, linear bivariate Pearson's correlation analyses were conducted between the GM volume extracted from the clusters of the brain regions with significant GM volume changes and the Matrix Reasoning Test direct scores. To obtain the GM volume change, we calculated the difference in ml from S1 to S2 in the clusters with significant changes. IBM SPSS 25 Statistics software (IBM, Chicago, IL, USA) was used to conduct the analyses. 


\section{Results}

Behavioral $n$-back fMRI and $n$-back training results as well as $n$-back task-fMRI analysis results can be found in supporting information.

\subsection{Voxel-Based Morphometry Results}

The data collected in S1 were used to perform a two-sample t test analysis in order to verify that the two groups did not differ in their GM volume before training. The results showed no significant differences. Consequently, the brain differences found between groups in subsequent sessions would not be due preexisting differences. The threshold was $\mathrm{p}<.05$ FWE, cluster-corrected using a threshold of $\mathrm{p}<.001$ at the uncorrected voxel level.

To study the sustained training effects on the GM volume, an interaction analysis was performed. This analysis assessed the GM volume changes from S1 to S2 and S3, in terms of increases or decreases in GM volume. When studying the sustained effects of the training [contrast: Trained group $(\mathrm{S} 1<\mathrm{S} 2, \mathrm{~S} 3)>$ Control group $(\mathrm{S} 1<\mathrm{S} 2, \mathrm{~S} 3)]$, we found one region that showed significant GM volume enlargement in the trained group in comparison with controls: the right superior parietal cortex (RSPC; Figure 2). The MNI coordinates for the maximum were $\mathrm{x}=15, \mathrm{y}=-40$, and $\mathrm{z}=72$, the Brodmann areas (BA) corresponded with 3, 5 and 7, and lastly, the $\mathrm{Z}$ and $\mathrm{T}$ values were 4.19 and 4.38, respectively. The reverse contrast yielded no significant effects.

Transient changes due to training were studied using the polynomic contrast. The contrast Control group $(\mathrm{S} 1, \mathrm{~S} 3<\mathrm{S} 2)>$ Trained group $(\mathrm{S} 1, \mathrm{~S} 3<\mathrm{S} 2)$ yielded a significant difference in the right putamen (Figure 2). In this case, the MNI coordinates for the maximum were 
$\mathrm{x}=39, \mathrm{y}=-26$, and $\mathrm{z}=4, \mathrm{BA} 48, \mathrm{Z}$-value $=4.75$, and $\mathrm{T}=5.03$. The opposite contrast did not yield any significant differences.

Figure 2: Brain areas with GM volume changes in the trained group after performing a specific brief $n$-back training. (A) Sustained changes (in yellow; Trained group ( $\mathrm{S} 1<\mathrm{S} 2$, S3) > Control group $(\mathrm{S} 1<\mathrm{S} 2, \mathrm{~S} 3))$ and Transient changes (in blue; Trained group (S1, $\mathrm{S} 3<\mathrm{S} 2)<$ Control group $(\mathrm{S} 1, \mathrm{~S} 3<\mathrm{S} 2))$. Results were $\mathrm{p}<.05 \mathrm{FWE}$, cluster-corrected, using a threshold of $\mathrm{p}<.001$ at the uncorrected voxel level and a cluster extension of $\mathrm{k}=666$ voxels and $\mathrm{k}=703$ voxels, respectively. Error bars represent standard errors. (B and C) GM volume (in $\mathrm{ml}$ ) evolution across sessions in the two groups in the right superior parietal cortex (RSPC; B) and in right putamen (C). Bars show mean values for each group. S1, S2, and S3 correspond to pre-training, post-training, and follow-up sessions, respectively. Green bars correspond to the training group, and blue bars correspond to the control group. $\mathrm{R}=$ right. $\mathrm{GM}=$ gray matter.
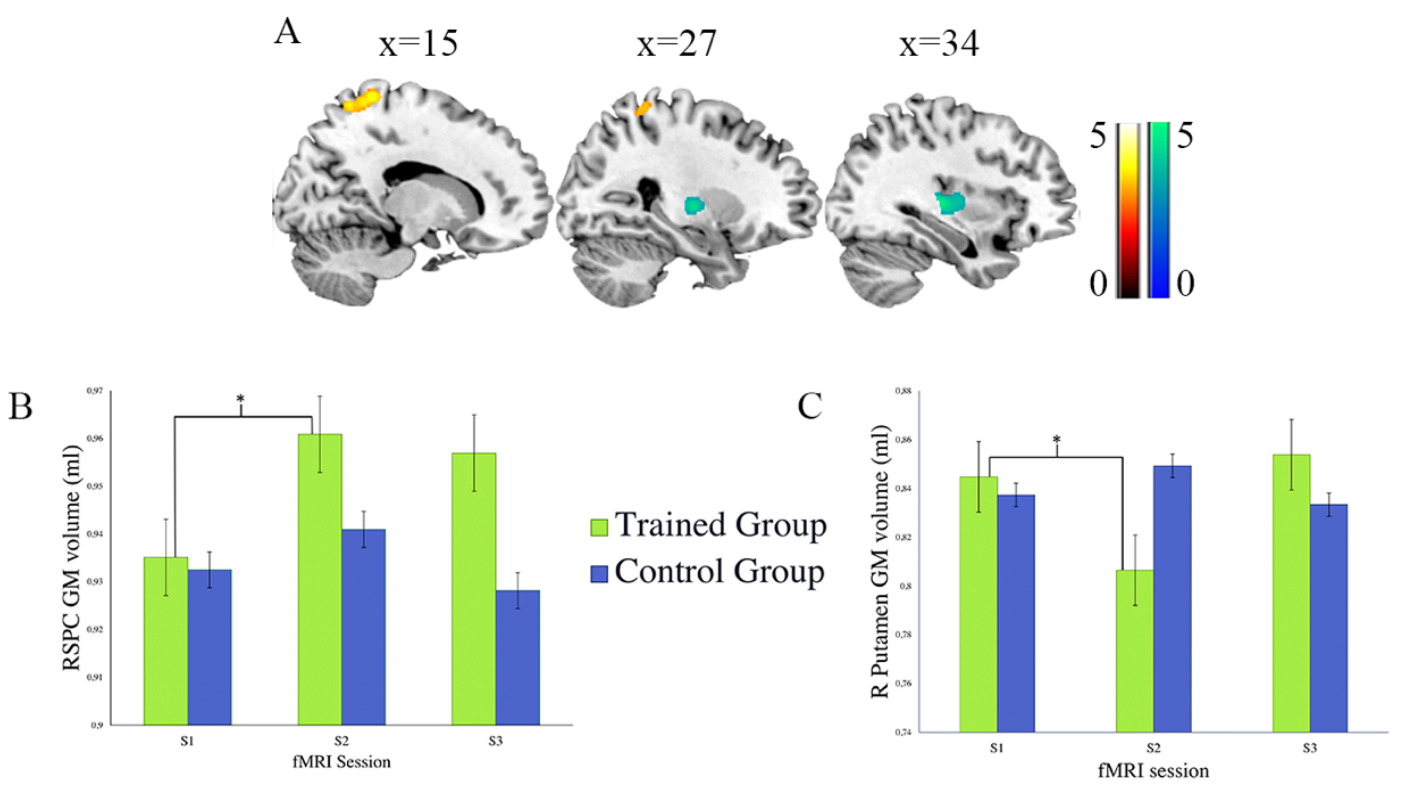

3.2. Correlation Analyses with intellectual level

The relationship between IQ scores and GM volume changes after training was studied by means of bivariate Pearson's correlation analysis. We used the two regions that had changes in GM volume, described above, and their MNI coordinates were used to extract the data and create the clusters. Then, the GM volume (in ml) was extracted from the clusters for each session, and the difference in $\mathrm{ml}$ from $\mathrm{S} 1$ to $\mathrm{S} 2$ was calculated. The 
results yielded a significant negative correlation between the GM volume change in the right putamen in S2 and the intellectual level $(\mathrm{r} 25=-0.46 \mathrm{p}<.05$; Control group: $\mathrm{r} 26=$ $0.01 \mathrm{p}=.97$; Figure 3). The difference between these two correlation coefficients was statistically significant (Trained group $>$ Control group, $\mathrm{z}=0.46 \mathrm{p}<.05$ one-tailed). When analyzing the relationship between the RSPC and the IQ level, the analysis did not show any significant correlations $(\mathrm{r} 25=0.38 \mathrm{p}=.57$ for the experimental group and $\mathrm{r} 26=-0.19$ $\mathrm{p}=.35$ for the control group; Figure 3).

Figure 3: Matrix Reasoning Test direct score relationships with the gray matter (GM) volume change (in $\mathrm{ml}$ ) from $\mathrm{S} 1$ to $\mathrm{S} 2$ in the areas where trained-related changes were found. (A) Right superior parietal cortex (RSPC) and (B) right Putamen. Circular green dots correspond to the training group, and square blue dots correspond to the control group. $\mathrm{R}$ = right.

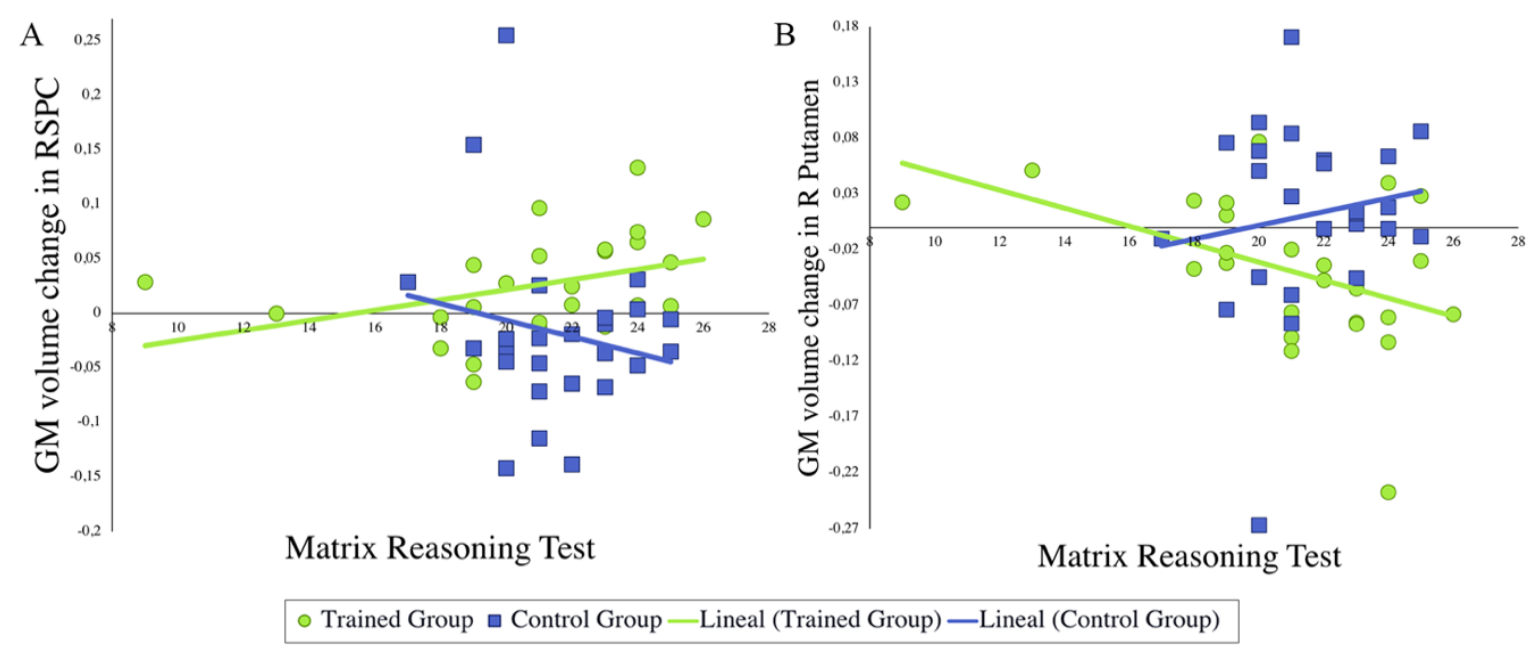

\section{Discussion}

Here we investigated the sustained and transient changes in GM volume after 200 minutes of an adaptive single $n$-back training and their relationship with behavioral improvements. Using the VBM method, we compared the GM volume of two groups (trained or passive control group) before training, immediately after training, and after five weeks of no training. Using this new three-time point approach, we found two 
significant changes due to training. We first obtained a sustained increment in the GM volume of the right superior parietal cortex. We also found a transient reduction in GM volume in the right putamen that correlated negatively with IQ scores. Our results demonstrate that a brief and intensive $n$-back training causes GM volume changes in brain areas involved in working memory processing.

As previously obtained in this sample, $n$-back training has been associated with behavioral improvements and decreased brain activation in frontoparietal areas (MiróPadilla et al., 2018). Behaviorally, we observed that most of the trained participants reached an asymptotic performance after training that was maintained after five weeks of no training. This ceiling effect keeps us from using improvements in performance measures as possible correlates of GM changes. Previous results also showed a sustained decrease in the activation of bilateral frontal and right parietal areas (including the right superior parietal cortex) after training. Thus, the functional response in the main frontoparietal areas presumably participated actively throughout the training process. By contrast, the basal ganglia did not participate significantly in the task during baseline, and they did not change their functional activity after training.

Consistent with our hypothesis, a sustained GM increase has been found in the right superior parietal gyrus, a brain area with a prominent role in working memory. The superior parietal cortex is not an essential area in the $n$-back task (Owen, McMillan, Laird, \& Bullmore, 2005). However, several studies have reported that BOLD activation in this area is a good positive predictor of behavioral performance (DeYoung, Shamosh, Green, Braver, \& Gray, 2009; Owens, Duda, Sweet, \& MacKillop, 2018; Zou et al., 2013), and that changes in BOLD activity were positively correlated with anatomic volume (Owens 
et al., 2018). This latter study, performed with more than 1000 individuals, additionally showed that this area had the strongest integration between BOLD activity during the task and GM volume. The role of this area in working memory has been related to spatial processing, but also to verbal and phonological stimuli (Collette et al., 2006; Nee et al., 2013). Overall, the superior parietal lobe is critically important for the top-down manipulation of all kinds of information, fixing the attention on relevant items (Humphreys \& Lambon Ralph, 2015; Koenigs, Barbey, Postle, \& Grafman, 2009; Nee et al., 2013).

The results of the present study also obtained a transient reduction in the volume of the posterior putamen that was not observable five weeks later. The striatum does not directly participate in working memory processing, but recent data suggest that this brain area may modulate the individual differences in working memory capacity and the effect of training by facilitating plasticity in the cortex (Constantinidis \& Klingberg, 2016; McNab \& Klingberg, 2007). Several previous studies have provided clues to help to understand the role of the striatum in working memory. First, there are developmental studies showing that striatal activity during working memory tasks is a good predictor of future (but not current) working memory capacity (Darki \& Klingberg, 2015; Ullman, Almeida, \& Klingberg, 2014). Second, a PET study showed that working memory training enhanced dopamine release in the striatum (Backman et al., 2011). Third, genetic studies showed the role of polymorphisms related to the DAT-1 transporter (Brehmer et al., 2009; Söderqvist et al., 2012) and DRD2 receptor expression (Söderqvist, Matsson, PeyrardJanvid, Kere, \& Klingberg, 2014) in individual differences in improvements after working memory training. Finally, and consistent with data from the present study, the role of the striatum is more relevant in the first stages of training (Kühn et al., 2013). All 
of this evidence is consistent with a prominent role of the striatum in the initial stages of working memory training, probably facilitating plasticity in frontoparietal areas. Importantly, this reduction in the volume was greater in participants with a lower score on the Matrix Reasoning Test. This result indicates that participants with high scores in this test had less GM volume in the right putamen after training. We tentatively interpret this result by saying that participants with a higher IQ level had fewer striatal changes because they did not need to adjust their cerebral resources to improve on the task. As pointed out before, the striatum modulates training changes in working memory; consequently, participants who have more cognitive resources (higher IQ scores) already have the required processing resources and do not need to readjust their GM volume as much as the participants with lower IQs to increase their accuracy and perform the task better.

Nevertheless, because of the lack of an active control group, these results should be interpreted with caution. The training group came to our laboratory on four consecutive days, and they had more contact with the experimenters than the control group did, which may result in motivational differences between the two groups in terms of task efficiency. In future studies, active control groups should be included in the study design because the observed gains may not be due to working memory training per se, but to the training in general.

In conclusion, having previously observed that an adaptive 200 minutes of single $n$-back training improves behavioral performance and causes cerebral modifications, as indicated by the decrease in the activation of diverse brain areas involved in working memory in the trained task and the transfer task (Miró-Padilla et al., 2018; submitted), here we extend 
our work by investigating the GM volume changes after this specific short training.

Sustained changes were found in the right superior parietal cortex, and transient changes were observed in the right putamen, both structures closely related to working memory. Increased GM volume was found in the RSPC in the trained participants, and the reverse effect was seen in the right putamen. We reported stability in the RSPC changes after five weeks of no training, and the results pointed to a relationship between the GM volume changes and the behavioral improvement and the IQ scores. Thus, the present results showed that short cognitive training can cause plasticity in brain structures related to the trained task, and they may support the neural efficiency theory because the greater the IQ level, the fewer the GM changes.

\section{References}

Backman, L., Nyberg, L., Soveri, A., Johansson, J., Andersson, M., Dahlin, E., ... Rinne, J. O. (2011). Effects of Working-Memory Training on Striatal Dopamine Release. Science, 333(6043), 718-718. https://doi.org/10.1126/science.1204978

Bengtsson, S. L., Nagy, Z., Skare, S., Forsman, L., Forssberg, H., \& Ullén, F. (2005). Extensive piano practicing has regionally specific effects on white matter development. Nature Neuroscience, 8, 1148. Retrieved from http://dx.doi.org/10.1038/nn1516

Brehmer, Y., Westerberg, H., Bellander, M., Fürth, D., Karlsson, S., \& Bäckman, L. (2009). Working memory plasticity modulated by dopamine transporter genotype. Neuroscience Letters, 467(2), 117-120. https://doi.org/10.1016/j.neulet.2009.10.018

Buschkuehl, M., Jaeggi, S. M., \& Jonides, J. (2012). Neuronal effects following working memory training. Developmental Cognitive Neuroscience, 2, Supplem, S167-S179. https://doi.org/10.1016/j.den.2011.10.001

Collette, F., Hogge, M., Salmon, E., \& Van der Linden, M. (2006). Exploration of the neural substrates of executive functioning by functional neuroimaging. Neuroscience, 139(1), 209-221. https://doi.org/10.1016/j.neuroscience.2005.05.035

Colom, R., Martínez, K., Burgaleta, M., Román, F. J., García-García, D., Gunter, J. L., ... Thompson, P. M. (2016). Gray matter volumetric changes with a challenging adaptive cognitive training program based on the dual n-back task. Personality and Individual Differences, 98, 127-132. https://doi.org/10.1016/J.PAID.2016.03.087

Constantinidis, C., \& Klingberg, T. (2016). The neuroscience of working memory capacity and training. Nature Reviews Neuroscience, 17(7), 438-449. https://doi.org/10.1038/nrn.2016.43

Dahlin, E., Neely, A. S., Larsson, A., Bäckman, L., \& Nyberg, L. (2008). Transfer of 
learning after updating training mediated by the striatum. Science (New York, N.Y.), 320(5882), 1510-1512. https://doi.org/10.1126/science.1155466

Darki, F., \& Klingberg, T. (2015). The Role of Fronto-Parietal and Fronto-Striatal Networks in the Development of Working Memory: A Longitudinal Study. Cerebral Cortex, 25(6), 1587-1595. https://doi.org/10.1093/cercor/bht352

DeYoung, C. G., Shamosh, N. A., Green, A. E., Braver, T. S., \& Gray, J. R. (2009). Intellect as Distinct From Openness: Differences Revealed by fMRI of Working Memory. Journal of Personality and Social Psychology, 97(5), 883-892. https://doi.org/10.1037/a0016615

Draganski, B., Gaser, C., Busch, V., Schuierer, G., Bogdahn, U., \& May, A. (2004). Changes in grey matter induced by training. Nature, 427, 311. Retrieved from http://dx.doi.org/10.1038/427311a

Draganski, B., Gaser, C., Kempermann, G., Kuhn, H. G., Winkler, J., Büchel, C., \& May, A. (2006). Temporal and Spatial Dynamics of Brain Structure Changes during Extensive Learning. The Journal of Neuroscience, 26(23), 6314. Retrieved from http://www.jneurosci.org/content/26/23/6314.abstract

Durstewitz, D., Seamans, J. K., \& Sejnowski, T. J. (2000). Dopamine-Mediated Stabilization of Delay-Period Activity in a Network Model of Prefrontal Cortex. Journal of Neurophysiology, 83(3), 1733-1750. https://doi.org/10.1152/jn.2000.83.3.1733

Edin, F., Klingberg, T., Johansson, P., McNab, F., Tegnér, J., \& Compte, A. (2009). Mechanism for top-down control of working memory capacity. Proceedings of the National Academy of Sciences of the United States of America, 106(16), 68026807. https://doi.org/10.1073/pnas.0901894106

Engvig, A., Fjell, A. M., Westlye, L. T., Moberget, T., Sundseth, Ø., Larsen, V. A., \& Walhovd, K. B. (2010). Effects of memory training on cortical thickness in the elderly. $\quad$ NeuroImage, 52(4), 1667-1676. https://doi.org/10.1016/J.NEUROIMAGE.2010.05.041

Gaser, C., \& Schlaug, G. (2003a). Brain Structures Differ between Musicians and NonMusicians. The Journal of Neuroscience, 23(27), 9240. Retrieved from http://www.jneurosci.org/content/23/27/9240.abstract

Gaser C Schlaug G. (2003b). Gray Matter Differences between Musicians and Nonmusicians. Annals of the New York Academy of Sciences, 999(1), 514-517. https://doi.org/10.1196/annals.1284.062

Golestani, N., \& Pallier, C. (2007). Anatomical Correlates of Foreign Speech Sound Production. Cerebral Cortex, 17(4), 929-934. Retrieved from http://dx.doi.org/10.1093/cercor/bhl003

Golestani, N., Paus, T., \& Zatorre, R. J. (2002). Anatomical Correlates of Learning Novel Speech Sounds. Neuron, 35(5), 997-1010. https://doi.org/10.1016/S08966273(02)00862-0

Golestani, N., \& Zatorre, R. J. (2004). Learning new sounds of speech: reallocation of neural substrates. NeuroImage, 21(2), 494-506. https://doi.org/10.1016/J.NEUROIMAGE.2003.09.071

Humphreys, G. F., \& Lambon Ralph, M. A. (2015). Fusion and Fission of Cognitive Functions in the Human Parietal Cortex. Cerebral Cortex, 25(10), 3547-3560. Retrieved from http://dx.doi.org/10.1093/cercor/bhu198

Hyde, K. L., Lerch, J., Norton, A., Forgeard, M., Winner, E., Evans, A. C., \& Schlaug, G. (2009). Musical Training Shapes Structural Brain Development. The Journal of Neuroscience, $\quad 29(10), \quad 3019 . \quad$ Retrieved from http://www.jneurosci.org/content/29/10/3019.abstract 
Ilg, R., Wohlschläger, A. M., Gaser, C., Liebau, Y., Dauner, R., Wöller, A., .. Mühlau, M. (2008). Gray Matter Increase Induced by Practice Correlates with Task-Specific Activation: A Combined Functional and Morphometric Magnetic Resonance Imaging Study. The Journal of Neuroscience, 28(16), 4210. Retrieved from http://www.jneurosci.org/content/28/16/4210.abstract

Jaeggi, S. M., Buschkuehl, M., Perrig, W. J., \& Meier, B. (2010). The concurrent validity of the N -back task as a working memory measure. Memory, 18(4), 394-412. https://doi.org/10.1080/09658211003702171

Klingberg, T. (2010). Training and plasticity of working memory. Trends in Cognitive Sciences, 14(7), 317-324. https://doi.org/10.1016/j.tics.2010.05.002

Koenigs, M., Barbey, A. K., Postle, B. R., \& Grafman, J. (2009). Superior parietal cortex is critical for the manipulation of information in working memory. The Journal of Neuroscience : The Official Journal of the Society for Neuroscience, 29(47), 1498014986. https://doi.org/10.1523/JNEUROSCI.3706-09.2009

Kühn, S., Schmiedek, F., Noack, H., Wenger, E., Bodammer, N. C., Lindenberger, U., \& Lövden, M. (2013). The dynamics of change in striatal activity following updating training. Human Brain Mapping, 34(7), 1530-1541. https://doi.org/10.1002/hbm.22007

Küper, K., \& Karbach, J. (2016). Increased training complexity reduces the effectiveness of brief working memory training: evidence from short-term single and dual $\mathrm{n}$-back training interventions. Journal of Cognitive Psychology, 28(2), 199-208. https://doi.org/10.1080/20445911.2015.1118106

Mårtensson, J., Eriksson, J., Bodammer, N. C., Lindgren, M., Johansson, M., Nyberg, L., \& Lövdén, M. (2012). Growth of language-related brain areas after foreign language learning. NeuroImage, 63(1), 240-244. https://doi.org/10.1016/J.NEUROIMAGE.2012.06.043

McNab, F., \& Klingberg, T. (2007). Prefrontal cortex and basal ganglia control access to working memory. Nature Neuroscience, 11, 103. Retrieved from http://dx.doi.org/10.1038/nn2024

McNab, F., Varrone, A., Farde, L., Jucaite, A., Bystritsky, P., Forssberg, H., \& Klingberg, T. (2009). Changes in Cortical Dopamine D1 Receptor Binding Associated with Cognitive Training. Science, 323(5915), 800-802. https://doi.org/10.1126/science.1166102

Metzler-Baddeley, C., Caeyenberghs, K., Foley, S., \& Jones, D. K. (2016). Task complexity and location specific changes of cortical thickness in executive and salience networks after working memory training. NeuroImage, 130, 48-62. https://doi.org/10.1016/j.neuroimage.2016.01.007

Miró-Padilla, A., Bueichekú, E., Ventura-Campos, N., Flores-Compañ, M. J., Parcet, M. A., \& Ávila, C. (2018). Long-term brain effects of N-back training: an fMRI study. Brain Imaging and Behavior, 1-13. https://doi.org/10.1007/s11682-018-9925-X

Nee, D. E., Brown, J. W., Askren, M. K., Berman, M. G., Demiralp, E., Krawitz, A., \& Jonides, J. (2013). A Meta-analysis of Executive Components of Working Memory. Cerebral Cortex, 23(2), 264-282. https://doi.org/10.1093/cercor/bhs007

Owen, A. M., McMillan, K. M., Laird, A. R., \& Bullmore, E. (2005). N-back working memory paradigm: a meta-analysis of normative functional neuroimaging studies. Human Brain Mapping, 25(1), 46-59. https://doi.org/10.1002/hbm.20131

Owens, M. M., Duda, B., Sweet, L. H., \& MacKillop, J. (2018). Distinct functional and structural neural underpinnings of working memory. NeuroImage, 174(December 2017), 463-471. https://doi.org/10.1016/j.neuroimage.2018.03.022

Palomar-García, M. Á., Zatorre, R. J., Ventura-Campos, N., Bueichekú, E., \& Ávila, C. 
(2017). Modulation of Functional Connectivity in Auditory-Motor Networks in Musicians Compared with Nonmusicians. Cerebral Cortex, 27(5), 2768-2778. https://doi.org/10.1093/cercor/bhw120

Salminen, T., Kühn, S., Frensch, P. A., \& Schubert, T. (2016). Transfer after Dual n-Back Training Depends on Striatal Activation Change. The Journal of Neuroscience, 36(39), 10198. Retrieved from http://www.jneurosci.org/content/36/39/10198.abstract

Sluming, V., Barrick, T., Howard, M., Cezayirli, E., Mayes, A., \& Roberts, N. (2002). Voxel-Based Morphometry Reveals Increased Gray Matter Density in Broca's Area in Male Symphony Orchestra Musicians. NeuroImage, 17(3), 1613-1622. https://doi.org/10.1006/NIMG.2002.1288

Söderqvist, S., Matsson, H., Peyrard-Janvid, M., Kere, J., \& Klingberg, T. (2014). Polymorphisms in the Dopamine Receptor 2 Gene Region Influence Improvements during Working Memory Training in Children and Adolescents. Journal of Cognitive Neuroscience, 26(1), 54-62. https://doi.org/10.1162/jocn_a_00478

Söderqvist, S., Nutley, S. B., Peyrard-Janvid, M., Matsson, H., Humphreys, K., Kere, J., \& Klingberg, T. (2012). Dopamine, working memory, and training induced plasticity: Implications for developmental research. Developmental Psychology, 48(3), 836-843. https://doi.org/10.1037/a0026179

Soveri, A., Antfolk, J., Karlsson, L., Salo, B., \& Laine, M. (2017). Working memory training revisited: A multi-level meta-analysis of n-back training studies. Psychonomic Bulletin \& Review. https://doi.org/10.3758/s13423-016-1217-0

Stein, M., Federspiel, A., Koenig, T., Wirth, M., Strik, W., Wiest, R., ... Dierks, T. (2012). Structural plasticity in the language system related to increased second language proficiency. Cortex, 48(4), 458-465. https://doi.org/10.1016/J.CORTEX.2010.10.007

Takeuchi, H., Taki, Y., \& Kawashima, R. (2010). Effects of working memory training on cognitive functions and neural systems. Reviews in the Neurosciences, 21(6), 427449. Retrieved from http://europepmc.org/abstract/MED/21438192

Takeuchi, H., Taki, Y., Sassa, Y., Hashizume, H., Sekiguchi, A., Fukushima, A., \& Kawashima, R. (2011). Working memory training using mental calculation impacts regional gray matter of the frontal and parietal regions. PLoS ONE, 6(8). https://doi.org/10.1371/journal.pone.0023175

Ullman, H., Almeida, R., \& Klingberg, T. (2014). Structural Maturation and Brain Activity Predict Future Working Memory Capacity during Childhood Development. Journal of Neuroscience, 34(5), 1592-1598. https://doi.org/10.1523/JNEUROSCI.0842-13.2014

Zou, Q., Ross, T. J., Gu, H., Geng, X., Zuo, X.-N., Hong, L. E., ... Yang, Y. (2013). Intrinsic resting-state activity predicts working memory brain activation and behavioral performance. Human Brain Mapping, 34(12), 3204-3215. 


\section{SUPPORTING INFORMATION}

\section{Behavioral results}

\section{$\underline{N \text {-back behavioral fMRI results }}$}

The repeated measures $2 \times 3 \times 3$ mixed-model ANOVA conducted for accuracy yielded main effects for Session $(F(2,50)=34.66 \mathrm{p}<.001)$ and Load Level $(\mathrm{F}(2,50)=42.85$ $\mathrm{p}<.001$ ), which means that all the participants reduced their mistakes in the post-training and follow-up sessions, compared to Session 1, and that the highest accuracy scores were observed during the 0-back. These main effects were driven by significant Group $\mathrm{x}$ Session $(\mathrm{F}(2,50)=7.77 \mathrm{p}=.001)$, Load Level x Session $(\mathrm{F}(4,48)=13.07 \mathrm{p}<.001)$, and Load Level x Group $(\mathrm{F}(2,50)=7.23 \mathrm{p}=.002)$ interactions. The first interaction indicated that trained participants were better than controls during the post-training and follow-up sessions. The second indicated that differences between load levels were greater at pretraining, whereas the third reflected that the training group showed better performance than the control group on 2-back and 3-back. As expected, the Load Level x Session $\mathrm{x}$ Group interaction reached significance $(\mathrm{F}(4,48)=4.01 \mathrm{p}=.007)$, which means that the trained group became more accurate in the post-training and follow-up sessions than the control group, when performing the 2-back and 3-back load levels. Post-hoc analyses revealed that these differences were significant for 3-back vs 0 -back $(\mathrm{p}=.002)$, and they only approached significance for 2-back vs 0 -back $(\mathrm{p}=.13)$.

Analyses of RTs scores revealed a similar pattern to the one found for accuracy. The $2 \times 3 \times 3$ ANOVA also yielded significant main effects for Session $(F(2,50)=51.59 \mathrm{p}<.001)$ and Load Level $(\mathrm{F}(2,50)=75.37 \mathrm{p}<.001)$. Both groups responded faster in the posttraining and follow-up sessions than in the pre-training session. Moreover, both responded faster in the 0-back load level compared to the 2-back load level, as well as in 
the 2-back load level compared to the 3-back load level. Significant two-way interactions were obtained for the Group x Session $(F(2,50)=28.14 \mathrm{p}<.001)$ and Load Level $x$ Session $(\mathrm{F}(4,48)=28.23 \mathrm{p}<.001)$ interactions. The first two interactions may be interpreted similarly to accuracy; participants were faster than controls during the post-training and follow-up, and the differences between load levels were greater at pre-training. Importantly, all these significant effects were qualified by the three-way Load Level $\mathrm{x}$ Session $x$ Group interaction, which was highly significant $(F(4,48)=11.34 p<.001)$. As expected, this interaction showed that the training group, compared to the controls, was faster after training and in the follow-up session in the 2-back and 3-back load levels. Post-hoc analyses revealed that this effect was significant for both 2-back vs 0-back and 3-back vs 0-back load levels ( $\mathrm{p}<.001)$.

\section{$\underline{N \text {-back behavioral training results }}$}

With the behavioral training data for the training group, a repeated-measures $2 \times 4$ ANOVA was conducted with the results of the test part of the training to evaluate their progress on $n$-back. For accuracy training performance, a main effect of Training Session $(\mathrm{F}(3,27)=6.49 \mathrm{p}<.05)$ and Load Level $(\mathrm{F}(1,29)=11.99 \mathrm{p}<.05)$ was found, indicating participants' improvement, in terms of correct answers from one training session to another, and reductions in their mistakes on both 2-back and 3-back. For RT values, we could see a significant effect of Training Session $(F(3,23)=10.35 p<.001)$, which means that subjects' RTs decreased from one training session to another. As expected, these results confirmed the great progress of the training group on $n$-back performance after 200 minutes of training.

\section{Functional results}

\section{$\underline{N \text {-back task-effects at baseline }}$}


A whole-brain one-sample $t$ test was conducted in order to study the brain regions involved in the $n$-back task (2-back and 3-back load levels $>0$-back load level). We used the fMRI data collected in Session 1. This analysis showed significant cortical and subcortical activations in brain areas related to working memory. Studying the task effects for each 2-back and 3-back load level (2-back>0-back and 3-back>0-back) separately, the same areas were activated: bilateral superior, middle and inferior frontal cortex (BA 6/8-11/32/45-48), including supplementary motor area/anterior cingulate gyrus (SMA/ACC) (BA 6/32) and the insula (BA 47), bilateral superior and inferior parietal cortex (BA 7/40), including the precuneus and bilateral cerebellum (crus I). Midbrain areas (thalamus and globus pallidus) were not significantly activated in 3-back, whereas they were in 2-back. Results were p<.05 FWE cluster-corrected using a threshold of $\mathrm{p}<.001$ at the uncorrected voxel level with a cluster extension of $\mathrm{k}=2504$ voxels for 2back and $\mathrm{k}=143$ for 3-back.

The two-sample $t$ test analysis performed between groups to examine the equality in brain responses in $\mathrm{S} 1$ yielded no significant functional differences. The threshold was $\mathrm{p}<0.001$ uncorrected at the voxel level.

\section{Training effects in $n$-back (trained task)}

To study the effects of training on the brain, an interaction analysis was conducted. Therefore, a 2x2 ANOVA (Group x Session) was carried out separately for each load level (2back and 3back). When studying the training effects by comparing S1 vs S2 and S1 vs S3 in the 2-back load level, we found similar results. These interaction analyses yielded activations in the bilateral superior frontal cortex (BA 8-9), including the SMA/ACC (BA 6/32), dorsolateral prefrontal cortex (BA 9/46), inferior frontal cortex 
(BA 44-46), and right inferior parietal cortex (IPC) (BA 39-40), in the trained group compared to the control group. The reverse contrast yielded no significant effects. Results were $\mathrm{p}<.05$ FWE cluster-corrected using a threshold of $\mathrm{p}<.001$ at the uncorrected voxel level and a cluster extension of $\mathrm{k}=125$ voxels and $\mathrm{k}=87$ voxels, respectively.

Regarding the 3-back load level, there were differences in the affected areas depending on the sessions compared. In the Trained group $(\mathrm{S} 1>\mathrm{S} 2)>$ Control group $(\mathrm{S} 1>\mathrm{S} 2)$ contrast, the analyses showed activations in the bilateral superior/middle frontal cortex (BA 8-11/46), including the SMA/ACC (BA 6/32), left insula, bilateral IPC (BA 39-40), and left temporal middle cortex (BA 21), in the trained group compared to the control group. On the other hand, in the Trained group $(\mathrm{S} 1>\mathrm{S} 3)>$ Control group $(\mathrm{S} 1>\mathrm{S} 3)$ contrast, a difference was found in the right IPC (BA 40), bilateral insula, SMA/ACC (6/32), bilateral inferior frontal cortex (BA 44-45), and dorsolateral prefrontal cortex (BA 9). The reverse contrasts yielded no significant differences. The threshold was at $\mathrm{p}<.05 \mathrm{FWE}$ cluster-corrected using an auxiliary threshold of $\mathrm{p}<.001$ at the uncorrected voxel level and a cluster extension of $k=89$ voxels and $k=67$ voxels, respectively.

When studying the stability of the effects of the working memory training over time, an interaction analysis was also conducted (Trained group $(\mathrm{S} 2>\mathrm{S} 3)>$ Control group (S2>S3)) separately for 2-back and 3-back. No significant effects were found in either load level or any comparison. The threshold was $\mathrm{p}<.001$ uncorrected at the voxel level. 


\section{CHAPTER 3. GENERAL DISCUSSION}

The present thesis aimed to study the behavioral and brain changes after working memory training. In order to accomplish this objective, we used the $n$-back task as the experimental paradigm for the training, and we collected behavioral and brain data by means of functional and structural MRI. Fifty-nine young healthy subjects participated in our study, and we randomly divided them into two groups: control and trained groups. We designed a training protocol for our trained group that consisted of four days of one hour of practice per day (200 minutes in total) during which participants completed an adaptive single $n$-back task. Both groups conducted three fMRI testing sessions: before training, immediately after training, and after five weeks of no training. In each fMRI session, participants completed the same adapted block-design $n$-back task (trained task) and the PASAT task (transfer task). Once the data had been collected, information was analyzed, and three experimental studies were developed. The main objective of the first study was to investigate, in terms of brain activation, the behavioral and cerebral trainingrelated effects during the performance of the trained task. The aim of the second study was to investigate the behavior and brain effects related to the $n$-back training on a different working memory task, in our case, the PASAT task, in order to study the far transfer effects. Finally, the objective of the third study was to investigate the sustained and transient changes in gray matter volume associated with this specific $n$-back training. All three aforementioned issues were studied in the short (immediately after finishing the training) and long-term (after five weeks of no training). Based on the results obtained, we have contributed more empirical evidence to the cognitive training field, and we have 
added new results indicating the effects of a short and intensive working memory training on behavioral and cerebral modifications that remain over time. Although each study contains a discussion of its results, this section will introduce a general overview of all the studies and examine and highlight common issues.

First and foremost, it is noteworthy that the three studies have related results because in all of them, training-related changes were found after the $n$-back training. First, brain activation changes were observed after training during the performance of both the trained and transfer tasks, and these changes were related to improvements on the tasks. Regarding the trained task, decreased brain activations in the trained participants were found in areas involved in working memory processes, such as the frontal superior/middle cortex, inferior parietal cortex, anterior cingulate cortex, and temporal middle cortex. Those decrements were globally accompanied by behavioral improvements on the $n$-back task; in other words, trained participants made fewer mistakes and were faster than the control group in giving the answers. Even though we observed non-significant improvements across the sessions in the control group due to the retest effect, the cerebral activation pattern of the control group remained constant across the three sessions. Moreover, while participants performed the transfer task, PASAT, decreased activations were also found in the trained group in the DLPFC (bilateral) compared to controls. These brain changes were related to the improvement on the task in the trained group, as indicated by the significant negative correlations found between PASAT improvements and the activations in LDLPFC. Our results demonstrated that these changes remained stable for at least five weeks because the same activation decreases in the same areas were found in the follow-up session. In addition, gray matter volume changes were observed in participants who trained 200 minutes, compared to the control group. An enlargement 
of gray matter volume was found in the right superior parietal cortex and remained stable five weeks later, and at the same time, a transient cluster of decreased gray matter volume was observed in the right putamen. These changes were related to the IQ level: a negative correlation was found between the right putamen and the Matrix Reasoning Test.

Within the working memory training field, behavioral studies are the most frequent. These investigations evaluated the improvements achieved by cognitive training on the training task and on different near and far transfer tasks. As previously stated in the introduction, given the large number of behavioral studies, many meta-analyses and reviews have been carried out to try to clarify and explain the results (Au, Buschkuehl, et al., 2015; Au, Sheehan, et al., 2015; Klingberg, 2010; Melby-Lervåg \& Hulme, 2013, 2016; Melby-Lervåg et al., 2016; Morrison \& Chein, 2011; Schwaighofer et al., 2015; Shipstead, Redick, \& Engle, 2012; Soveri et al., 2017; von Bastian \& Oberauer, 2014). Therefore, researchers agree that $n$-back is the most widely used task for working memory training studies, and it has been widely demonstrated that adequate $n$-back training improves task performance in terms of accuracy and reaction times, even with relatively short-term training. Generally, participants double or triple their pre-training performance levels. Our behavioral training-related behavioral results presented in this thesis followed along the same lines: participants who trained significantly improved their performance more than the control group in terms of accuracy and RTs on the $n$-back task, in most of cases reaching a ceiling performance. Importantly, these behavioral improvements remained stable after 5 weeks of no training or contact with the training task, coinciding with previous studies that held a follow up (2-8 months) session (Jaeggi, Buschkuehl, Jonides, \& Shah, 2011; Jaeggi, Buschkuehl, Shah, \& Jonides, 2014; Katz et al., 2017; Li et al., 2008). 
Special mention should be made of the behavioral transfer effects. As reviewed before, no clear conclusions could be extracted from the previous literature. Several studies have reported near and far transfer effects (Buschkuehl, Hernandez-Garcia, Jaeggi, Bernard, \& Jonides, 2014; Jaeggi et al., 2011; Jaeggi et al., 2010b; Schweizer, Grahn, Hampshire, Mobbs, \& Dalgleish, 2013), others found only near transfer effects (Harrison et al., 2013; Minear et al., 2016; Salminen et al., 2012; Waris et al., 2015), and others did not find any kind of transfer (Chooi \& Thompson, 2012; Clark et al., 2017b; Redick \& Lindsey, 2013; Thompson et al., 2013). The behavioral results of our second experiment adds to others that have failed to observe behavioral far transfer effects after working memory training. Our data showed no significant differences between groups in PASAT improvement after $n$-back training. Both groups improved in the post-training session, and this enhancement remained stable in the follow-up session. It has been demonstrated that repeated exposure to the PASAT improves performance (Correia, 2011; Tombaugh, 2006). Thus, the retest effect could be the cause of the equal improvement in both groups.

In the first and second report of this thesis, we used two different activation patterns, one for $n$-back and the other for PASAT. Both coincided with previous neuroimaging studies (Audoin et al., 2003; Forn et al., 2011; Owen et al., 2005) and strongly overlap each other. The brain activations were observed in the superior, middle, and inferior frontal cortex, including SMA, ACC and the anterior insula, the superior and inferior parietal cortex, including precuneus, inferior temporal cortex, cerebellum, and the thalamus and globus pallidus, all in both hemispheres. All the described areas are involved in different working memory processes. As is already known, PASAT and $n$-back are working memory tasks where participants have to maintain and manipulate verbal information during a short period of time and give a response. The difference between the two lies in the kind of 
manipulation (in PASAT they have to add simple numbers and in $n$-back they have to retain different number of letters) and the type of response (verbal or manual). Some of the shared activated areas related to working memory may involve different processes given these differences. The parietal cortex has frequently been associated with calculation and arithmetic procedure tasks (Dehaene, Piazza, Pinel, \& Cohen, 2003), crucial for PASAT performance, but it has also been related to the maintenance of verbal information (Collette \& Van Der Linden, 2002) as well as phonological storage (Baldo \& Dronkers, 2006; Tombaugh, 2006), useful for $n$-back. In addition, the SMA has been related to planning sequences of movement and motor activation of the hand (needed in $n$-back to give the answer), but at the same time, this area has been associated with the recruitment of the phonological loop and with executive functions (Wager \& Smith, 2003). Regarding the ACC and the insula, they are responsible for attention modulation, error detection, and motivation (Bush et al., 2000; Menon \& Uddin, 2010). The frontal areas, largely related to working memory, are in charge of executive system activity (Lara \& Wallis, 2015). The prefrontal cortex has the control of cognitive processing, stimuli selection, attention focus, and adequate responses. The thalamus and the cerebellum help the prefrontal cortex in its working memory functions due to their attentional role of filtering relevant information (thalamus) and assuming cognitive information processing functions (cerebellum) (Keren-Happuch et al., 2012; Watanabe \& Funahashi, 2012). That being said, the general neural overlap represents the common use of the working memory network, but the cognitive processes involved in each task only have in common the need to maintain verbal information, a process more related to the central executive component of working memory. 
Two major results have been reported in the present study regarding training-related brain changes observed immediately after training, that is, in the post-training session: decreased activation was observed in frontoparietal areas while conducting the trained task in participants who trained, and our findings showed reduced activation in frontal regions during performance of the transfer task in the trained group. Participants who trained for 200 minutes on our adaptive $n$-back task presented less activation in bilateral SFC, IPC, SMA, ACC while conducting 2-back, compared to the control group. Additionally, during the most demanding load level (3-back) performance, the same cerebral areas as in 2-back plus, the left insula and LMTC, presented reduced activation in the trained group. These findings are consistent with the literature that reports lower activation in brain areas related to working memory straight after an $n$-back training (Chang et al., 2017; Clark et al., 2017a; Salminen et al., 2016; Schneiders et al., 2011, 2012; Schweizer et al., 2013; Thompson et al., 2016). Moreover, while participants performed the transfer task, a decrease in the activation was observed in the anterior DLPFC (bilaterally) in the trained group, compared to the group that did not receive any kind of training. To our knowledge, this is the first study to show that neural transfer effects were located in crucial areas involved in working memory, such as the anterior prefrontal cortex. Previous investigations differ significantly from our results because they report neural near transfer in the striatum (Dahlin, Neely, Larsson, Bäckman, \& Nyberg, 2008; Salminen et al., 2016). The striatum is involved in this specific transfer because it requires the regulation of which information is relevant for the working memory task. This process is not involved in the far transfer in our experiment. Both prior works utilized two similar tasks that require learning certain cognitive processes, such as letter updating (Dahlin et al., 2008) or dual-task working memory (Salminen et al., 2016), which are transferred from the trained task to the transfer task. In our case, the PASAT is 
a very difficult task with a clear component of cognitive control (involving maintenance of stimuli and probably inhibition of responses), and this is probably the process transferred from $n$-back training.

The function of the prefrontal cortex in working memory is well-known. Updating and monitoring information, stimuli selection and inhibition, attention focus and switching, coordinating and integrating information, and providing adequate responses are examples of executive functions linked to prefrontal cortex activity (Alvarez \& Emory, 2006; Lara \& Wallis, 2015; Postle, 2006; Stuss \& Alexander, 2000). In the specific case of the DLPFC, due to its assorted connections with other brain areas, this prefrontal region has a crucial role in reasoning, monitoring, and manipulating cognitive items (Balconi, 2013; Barbey et al., 2013; Eriksson et al., 2015; Petrides, 2005; Petrides, 2000). In particular, the anterior DLPFC has a role as central executive in working memory, with flexible functions that operate at an abstract level modulating the processing of posterior brain structures (Constantinidis \& Klingberg, 2016; D’Esposito \& Postle, 2015). The insula also plays a role in inhibition (Wager et al., 2005), and it has been related to temporary detection of relevant stimuli, salience, and attention (Cauda et al., 2011), task-set maintenance (Dosenbach et al., 2007), and starting attentional control signals working together with the ACC (Menon \& Uddin, 2010). Meanwhile, the SMA is involved in planning sequences of movement, motor learning, and motor activation of the hand. Therefore, the decreased activation of these areas was expected because they have been widely related to the processes required to successfully perform the $n$-back. It should be emphasized that the area where we found decreased activation during the transfer task (DLPFC) overlapped with the $n$-back, indicating that this area is involved in both tasks. The anterior DLPFC controls the maintenance of verbal information for a few seconds, 
and this cognitive process is the only one in common between $n$-back and PASAT. Therefore, in light of the outcome, we have verified the hypothesis that transfer effects occur if the training and the transfer task involve the same brain regions and cognitive processes.

In addition to the brain activation changes, significant gray matter volume differences have been found after training between the two groups. Gray matter volume changes were seen in participants who belonged to the trained group: sustained changes were seen with an enlargement of gray matter volume in the RSPC and the transient effects, observing a reduction in gray matter volume in the right putamen. The stable change in the RSPC was expected because of the continuous use of this area during training and the maintenance of behavioral improvements. The RSPC has been related to top-down control of attention, numerical calculation (Humphreys \& Lambon Ralph, 2015), object location and verification (Rottschy et al., 2012), distractor resistance, updating (Nee et al., 2013), and ordering and manipulation of acquired facts (Koenigs et al., 2009), as shown in the literature on neuroimaging studies with healthy and clinical populations. In addition, the activation of the superior parietal cortex is a good positive predictor of behavioral performance, as different studies have pointed out (DeYoung, Shamosh, Green, Braver, \& Gray, 2009; Owens, Duda, Sweet, \& MacKillop, 2018; Zou et al., 2013). Owens and colleagues (2018) concluded in their study that changes in superior parietal BOLD activity were positively correlated with anatomic volume and showed that this area had the strongest integration between BOLD activity during the task and GM volume. Regarding the temporary change found in the striatum, it was also expected due to its role in facilitating cortical plasticity without participating in retaining in the working memory processes. The striatum acts as a relevant information filter in working memory ( $\mathrm{McNab}$ 
\& Klingberg, 2007), and, at the same time, a modulator role has been given to this cerebral structure in working memory, facilitating plasticity in frontoparietal networks and mediating the effects of working memory training (Constantinidis and Klingberg, 2016). This modulation activity could be due to changes in the dopamine system. Training in working memory increase dopamine release in the striatum, as a PET study pointed out (Backman et al., 2011), and genetic studies showed the role of polymorphisms related to the DAT-1 transporter (Brehmer et al., 2009; Söderqvist et al., 2012) and DRD2 receptor expression (Söderqvist, Matsson, Peyrard-Janvid, Kere, \& Klingberg, 2014) in individual differences in improvement after working memory training. Furthermore, another study by Kühn et al. (2013) claimed that the role of the striatum is more relevant in the first stages of training, which fits our results. The negative relationship observed between the gray matter volume change in the right putamen and the Matrix Reasoning Test scores in the trained group supports the idea that the striatum modulates training changes in working memory; therefore, participants who had more cognitive resources (higher IQ scores) had the required processing resources, and they did not need to modify their GM volume as much as the participants with lower IQ to enhance their performance on the task.

Now then, what does an activation decrease in neural activation after training mean? Commonly, decreased activation has been interpreted in functional neuroimaging based on the brain efficiency hypothesis. The brain (or neural) efficiency hypothesis postulates that individuals who perform cognitively demanding tasks better than others showed more efficient brain functioning. In this particular case, lower cortical activation could suggest a more efficient use of the brain in trained participants (Haier, Siegel, Tang, Abel, \& Buchsbaum, 1992; Neubauer \& Fink, 2009). According to scientific research, this 
phenomenon has been observed in different studies that utilized diverse neurophysiological measurement methods and a wide variety cognitive task demands (Neubauer \& Fink, 2009). Kelly et al. (2006) noted that this effect of reduced activation is typically observed after training on higher cognitive tasks, and they stated that lower activation is associated with increased neural efficiency, which means that less brain activity is needed to give a fast and accurate answer to the task. In the specific field of working memory training, lower activation in brain areas related to the task are understood as an indicator of neural efficiency. This decline in cerebral activation may allow participants to respond faster and make fewer mistakes (Buschkuehl et al. 2014).

Therefore, in light of the results obtained, it could be argued that working memory training produces an increase in brain efficiency, as signaled by the decreased activations found in the trained group in frontoparietal areas, an those decrements were accompanied by behavioral improvements on the trained task. Regarding the transfer task, the behavioral improvements were not as large as on $n$-back. However, our results showed a correlation in the trained group between the decrease in activation on the DLPFC and the improvement on the PASAT task after training, whereas this relationship was not observed in the control group. This result is consistent with the neural efficiency interpretation, where less bright participants will use more brain resources to perform a task than smarter participants. The participants in the trained group with a greater decrement were those with better performance. In other words, trained participants seemed to need fewer brain resources and less effort to make fewer mistakes. Because the DLPFC is involved in top-down control (Edin et al., 2009), the decrement probably reflects a lower requirement for cognitive control after training to attain a correct response. Importantly, the gray matter training-related changes were related to the 
participants' intellectual level. A significant negative relationship was observed between the gray matter volume change in the right putamen and the Matrix Reasoning Test scores. This relationship was found only in the trained group and indicates that participants with lower IQ scores presented the highest GM volume decrement in the right putamen. These results could also be interpreted according to the neural efficiency hypothesis. In this specific case, participants with a higher IQ level have the necessary cognitive processes to perform the task, and they did not have to modify their structures as much as the participants with lower IQs to successfully perform the $n$-back.

Nevertheless, recent views have a critical vision of the interpretation based on the neural efficiency hypothesis, classifying it as simple and unclear (Constantinidis \& Klingberg, 2016; Poldrack, 2015). Poldrack (2015) claimed critically that efficiency is not a useful concept in cognitive neuroscience. He viewed efficiency as inverted energy for the transmission of information in the brain networks. In addition, he highlighted the need for new studies and models to examine the neural changes, and he reported that identifying potential activation effects may lead to future mechanistic explanations. Therefore, although a decrease in activation is often interpreted as an increase in neural efficiency in the literature, our data did not demonstrate the underlying cellular mechanism, and so we cannot empirically show that decreased activation means better neural efficiency. Instead, our results point to the areas of change after working memory training that may lead to continue investigating mechanistic explanations in the future.

In the present thesis, we took a step further and investigated what happened with the $n$ back training-related neural effects in our trained and transfer task time after the training ended. As far as we are aware, it is the first study to investigate this in transfer tasks and 
the second in trained tasks. After 5 weeks of no training or contact with the training task, trained participants could have lost the training effects and returned to baseline brain response or maintained the training effects. Our results showed that the decreased activation in the same areas (for both training and transfer tasks) that occurred between the pre-training and post-training sessions was still present when comparing the pretraining session with the follow-up session. The main effect was stable five weeks after finishing the training. When the post-training session and the follow-up session were compared, no differences were found; in other words, brain activation did not increase again in the follow-up session or return to baseline levels. The stability of these brain changes after 5 weeks could suggest an improved efficiency of these areas because we found stability in the behavioral improvements on the tasks as well. Our data point in the same direction as the results reported by Chang et al., (2017), who observed that lower brain activity after $n$-back training remained stable on the trained task six months after finishing the training. Therefore, our results confirm that 200 minutes of working memory training produced cerebral modifications that persisted after weeks with no training. In addition, after observing the stability of the behavioral improvements and brain trainingrelated and transfer activation changes described above, stability of the gray matter volume changes after five weeks without training was expected. The sustained changes found in the RSPC confirmed this hypothesis: the gray matter volume changes in this parietal area remained stable after 5 weeks of no training. Therefore, this is the first time that stability of neural transfer effects and gray matter changes have been demonstrated.

In conclusion, a brief and intensive working memory training caused behavioral and brain changes that remained stable 5 weeks after finishing the training. Functional and structural changes were found in cerebral areas related to working memory, and these 
changes were related to improvements on the task. The cerebral changes were not only found during the performance of the trained task, but also during the execution of a different working memory task and in the gray matter volume. These results point in the direction of the neural efficiency hypothesis because the greater the improvements were, the fewer the brain activations and GM changes. In addition, the results also point in the direction of the hypothesis that transfer will occur if the training and transfer tasks involve the same brain regions and processing components, and they give the striatum a modulator role, mediating the effects of working memory training. 


\section{* General conclusions}

The general conclusions drawn in the present thesis are:

1. $N$-back and PASAT performance engages the same working memory brain areas. The two tasks differ substantially in the type of stimuli, the kind of cognitive manipulations, and the type of response. Thus, the neural overlap represents the common use of the working memory network, but cognitive processes involved in each task only share the need to maintain verbal information, a process more related to the central executive component of working memory.

2. A brief and intensive 200 minutes of working memory training leads to behavioral and neural modifications on the trained task. Trained participants significantly improve their performance, increasing accuracy and decreasing RTs. These behavioral changes in trained participants were associated with decreased activation in key areas for working memory (bilateral superior frontal cortex, IPC, SMA, ACC, left insula and left MTC) in participants who perform the training. Finally, these behavioral and neural advantages persist after five weeks of no training. Therefore, we conclude that cognitive training was associated with an improvement in behavioral performance and decreased brain activation, suggesting better neural efficiency that persists over time.

3. A brief and intensive 200 minutes of working memory training leads to neural transfer effects. Decreased activation in the area in charge of the process that both tasks share (DLPFC) was observed in participants who performed the training. At the same time, the trained group showed a negative relationship between the decrease in DLPFC and the PASAT improvement after training. Once more, the reductions observed after training persisted after five weeks. Thus, the decrement probably reflects a lower requirement of cognitive control after training to attain a correct response, and the results point in the direction of the hypothesis that transfer will occur if the training and the transfer tasks involve the same brain regions and processing components.

4. A brief and intensive 200 minutes of working memory training leads to gray matter volume changes. On the one hand, sustained changes were observed in the RSPC, 
which showed a significant gray matter volume enlargement. On the other hand, transient changes due to training were found in the right putamen. This subcortical region showed a decrease in its gray matter volume. These results were negative related to the IQ level. They may support the neural efficiency theory and the modulating role of the striatum in working memory because the higher the IQ level, the fewer the GM changes. 
In the present thesis, behavioral and brain effects of a brief working memory training have been studied in the short and long-term. On the one hand, we investigated the behavioral and cerebral changes on the trained and transfer tasks. On the other hand, the gray matter volume changes, and its relationship with improvements on the tasks have been studied. We have contributed more empirical evidence to the cognitive training field and, importantly, we have added new interesting neuroimaging results. Even so, further investigations are needed, and there is still a long way to go before the mechanisms of working memory and its training can be clarified. Therefore, working memory training research has not been finished yet, and here we present some of the experimental issues that could be answered:

* Are there any functional connectivity changes in the brain after this short working memory training?

Few such studies have been carried out to study the functional connectivity changes in the brain after working memory training. These previous studies pointed to increased frontoparietal network connectivity after training. It would be important to study what happens in the brain in terms of functional connectivity after this specific $n$-back training during the trained and the transfer task. In addition, research is needed to verify whether those possible functional connectivity changes are related to the behavioral task. Furthermore, those potential changes and their relationship with performance have to be studied in the long-term (five weeks or more), after the training is over. 
* Does the brain change in resting-state after this specific $n$-back training?

For years, the existence of brain activity without performing any cognitive task (i.e., intrinsic or spontaneous brain activity) was considered very low frequency random noise and, therefore, excluded. However, neuroimaging studies have shown that this activity is not random, but rather well-structured and organized. This resting-state activity has a similar amplitude to what appears during task performance, and it covers the entire brain cortex. A further step in research on this spontaneous activity is to give a "cognitive value" to this information and, as mentioned in the introduction, one of the most important cellular mechanisms of plasticity consists of the changes in connectivity while the brain is performing an activity and when it is not doing any task, that is at rest.

The scarce literature using fMRI and magnetoencephalography pointed to increased frontoparietal network connectivity after training, which positively correlated with the performance on the trained task. Therefore, it would be relevant to investigate the changes in functional connectivity in a resting-state after this brief and intensive working memory training.

* What happens to these brain activity changes after longer than five weeks? Extended follow-up (more than 5 weeks).

The present thesis has shown that trained participants presented a decreased activation in frontoparietal areas after 200 minutes of training, and these changes remained stable after five weeks with no training. It would be interesting and necessary to carry out research to investigate the long-term effects in longer retest periods in order to observe if the training effects remain, for example, three, six, or even twelve months later. 
* Does this kind of training have effects and benefits in clinical populations with working memory deficits?

It would be extremely important to increase the translation from basic to clinical neuroimaging investigations. Cognitive training effects are usually studied with healthy populations. This is a good point of departure because models of brain functioning can be developed by studying the intact brain. However, preventive and neurorehabilitation studies with elderly and clinical populations are still scarce, for instance, studies on the benefits of working memory training. Thus, the results from this kind of research could improve cognitive training and rehabilitation programs. Additionally, our training protocol was designed thinking about future clinical interventions with a short training period that could be less expensive for patients and institutions than other training protocols that last several months. 
Aben, B., Stapert, S., \& Blokland, A. (2012). About the distinction between working memory and short-term memory. Frontiers in Psychology, 3(AUG), 1-9. https://doi.org/10.3389/fpsyg.2012.00301

Allen, R. J., Baddeley, A. D., \& Hitch, G. J. (2014). Evidence for two attentional components in visual working memory. Journal of Experimental Psychology: Learning, Memory, and Cognition, 40(6), 1499-1509. https://doi.org/10.1037/xlm0000002

Alvarez, J. A., \& Emory, E. (2006). Executive Function and the Frontal Lobes: A MetaAnalytic Review. Neuropsychology Review, 16(1), 17-42. https://doi.org/10.1007/s11065-006-9002-x

Andreas, J., Agnes, F., Jutta, V. R., Carsten, K., Michael, R., Ann-Freya, F., ... Stefan, K. (2004). Crossed cerebro-cerebellar language dominance. Human Brain Mapping, 24(3), 165-172. https://doi.org/10.1002/hbm.20077

Anguera, J. A., Bernard, J. A., Jaeggi, S. M., Buschkuehl, M., Benson, B. L., Jennett, S., ... Seidler, R. D. (2012). The effects of working memory resource depletion and training on sensorimotor adaptation. Behavioural Brain Research, 228(1), 107-115. https://doi.org/10.1016/j.bbr.2011.11.040

Aron, A. R. (2011). From Reactive to Proactive and Selective Control: Developing a Richer Model for Stopping Inappropriate Responses. Biological Psychiatry, 69(12), e55-e68. https://doi.org/10.1016/J.BIOPSYCH.2010.07.024

Aron, A. R., Robbins, T. W., \& Poldrack, R. A. (2004). Inhibition and the right inferior frontal cortex. Trends in Cognitive Sciences, 8(4), 170-177. https://doi.org/10.1016/J.TICS.2004.02.010

Astle, D. E., Barnes, J. J., Baker, K., Colclough, G. L., \& Woolrich, M. W. (2015). Cognitive Training Enhances Intrinsic Brain Connectivity in Childhood. The Journal of Neuroscience, 35(16), 6277. Retrieved from http://www.jneurosci.org/content/35/16/6277.abstract

Atkinson, R. C., \& Shiffrin, R. M. (1968). Human memory: A proposed system and its control processes. (I. K. W. Spence, Ed.), The psychology of learning and motivation: Advances in research and theory (Vol. 2). New York: Academic Press.

Au, J., Buschkuehl, M., Duncan, G. J., \& Jaeggi, S. M. (2015). There is no convincing evidence that working memory training is NOT effective: A reply to Melby-Lervåg and Hulme (2015). Psychonomic Bulletin \& Review, 3(1), 331-337. https://doi.org/10.3758/s13423-015-0967-4

Au, J., Sheehan, E., Tsai, N., Duncan, G. J., Buschkuehl, M., \& Jaeggi, S. M. (2015). Improving fluid intelligence with training on working memory: a meta-analysis. Psychonomic Bulletin \& Review, 22(2), 366-377. https://doi.org/10.3758/s13423014-0699-x

Audoin, B., Ibarrola, D., Duong, M. V. A., Pelletier, J., Confort-Gouny, S., Malikova, I., ... Ranjeva, J.-P. (2005). Functional MRI study of PASAT in normal subjects. Magnetic Resonance Materials in Physics, Biology and Medicine, 18(2), 96-102. https://doi.org/10.1007/s10334-004-0098-9

Audoin, B., Ibarrola, D., Ranjeva, J. P., Confort-Gouny, S., Malikova, I., Ali-Chérif, A., 
... Cozzone, P. (2003). Compensatory cortical activation observed by fMRI during a cognitive task at the earliest stage of MS. Human Brain Mapping, 20(2), 51-58. https://doi.org/10.1002/hbm.10128

Awh, E., \& Jonides, J. (2001). Overlapping mechansims of attention and spatial working memory. Trends in Cognitive Sciences, 5(3), 119-126. https://doi.org/10.1016/S1364-6613(00)01593-X

Axmacher, N., Mormann, F., Fernández, G., Cohen, M. X., Elger, C. E., \& Fell, J. (2007). Sustained Neural Activity Patterns during Working Memory in the Human Medial Temporal Lobe. The Journal of Neuroscience, 27(29), 7807. Retrieved from http://www.jneurosci.org/content/27/29/7807.abstract

Backman, L., Nyberg, L., Soveri, A., Johansson, J., Andersson, M., Dahlin, E., ... Rinne, J. O. (2011). Effects of Working-Memory Training on Striatal Dopamine Release. Science, 333(6043), 718-718. https://doi.org/10.1126/science.1204978

Baddeley, A. (1992). Working memory. Science, 255(5044), 556-559. https://doi.org/10.1126/science.1736359

Baddeley, A. (1996). Exploring the Central Executive. The Quarterly Journal of Experimental Psychology Section A, 49(1), 5-28. https://doi.org/10.1080/713755608

Baddeley, A. (1996). The fractionation of working memory. Proceedings of the National Academy of $\quad$ Sciences, 93(24), 13468-13472. https://doi.org/10.1073/pnas.93.24.13468

Baddeley, A. (2000). The episodic buffer-a new component of working memory? Trends in Cognitive Science, 4(11), 417-423.

Baddeley, A. (2003a). Working memory: looking back and looking forward. Nature Reviews Neuroscience, 4(10), 829-839. https://doi.org/10.1038/nrn1201

Baddeley, A. (2003b). Working memory and language: An overview. Journal of Communication Disorders, 36(3), 189-208. https://doi.org/10.1016/S00219924(03)00019-4

Baddeley, A. (2006). Working memory: an overview. In Working Memory and Education. (S Pickerin, pp. 1-31). New York: Academic Press.

Baddeley, A. (2012). Working Memory: Theories, Models, and Controversies. Annual Review of Psychology, 63(1), 1-29. https://doi.org/10.1146/annurev-psych-120710100422

Baddeley, A. D., Allen, R. J., \& Hitch, G. J. (2011). Binding in visual working memory: The role of the episodic buffer. Neuropsychologia, 49(6), 1393-1400. https://doi.org/10.1016/j.neuropsychologia.2010.12.042

Baddeley, A. D., Baddeley, H. A., Bucks, R. S., \& Wilcock, G. K. (2001). Attentional control in Alzheimer's disease. Brain, 124(Pt 8), 1492-1508. https://doi.org/10.1093/brain/124.8.1492

Baddeley, A. D., \& Wilson, B. (1985). Phonological Coding and Short-Term-Memory in Patients without Speech. Journal of Memory and Language, 24(4), 490-502. https://doi.org/Doi 10.1016/0749-596x(85)90041-5

Baddeley, A., Gathercole, S., \& Papagno, C. (1998). The phonological loop as a language learning device. Psychological Review, 105(1), 158-173. https://doi.org/10.1037/0033-295X.105.1.158

Balconi, M. (2013). Dorsolateral prefrontal cortex, working memory and episodic memory processes: insight through transcranial magnetic stimulation techniques. Neuroscience Bulletin, 29(3), 381-389. https://doi.org/10.1007/s12264-013-1309-z

Baldo, J. V, \& Dronkers, N. F. (2006). The role of inferior parietal and inferior frontal cortex in working memory. Neuropsychology, 20(5), 529-538. 
https://doi.org/10.1037/0894-4105.20.5.529

Barbey, A. K., Koenigs, N., \& Grafman, J. (2013). Dorsolateral Prefrontal Contributions to Human Working Memory. Cortex, 49(5), 1195-1205. https://doi.org/10.1016/j.cortex.2012.05.022.Dorsolateral

Beatty, E. L., Jobidon, M.-E., Bouak, F., Nakashima, A., Smith, I., Lam, Q., ... Vartanian, O. (2015). Transfer of training from one working memory task to another: behavioural and neural evidence. Frontiers in Systems Neuroscience, 9(June), 86. https://doi.org/10.3389/fnsys.2015.00086

Bengtsson, S. L., Nagy, Z., Skare, S., Forsman, L., Forssberg, H., \& Ullén, F. (2005). Extensive piano practicing has regionally specific effects on white matter development. Nature Neuroscience, 8, 1148. Retrieved from http://dx.doi.org/10.1038/nn1516

Berryhill, M. E., Chein, J., \& Olson, I. R. (2011). At the intersection of attention and memory: the mechanistic role of the posterior parietal lobe in working memory. Neuropsychologia, 49(5), 1306-1315. https://doi.org/10.1016/j.neuropsychologia.2011.02.033

Braver, T. S. (2006). Working Memory. Cognitive Psychology: Mind and Brain. Retrieved from http://www-psych.stanford.edu/ ashas/Cognition Textbook/chapter6.pdf

Brehmer, Y., Westerberg, H., Bellander, M., Fürth, D., Karlsson, S., \& Bäckman, L. (2009). Working memory plasticity modulated by dopamine transporter genotype. Neuroscience Letters, 467(2), 117-120. https://doi.org/10.1016/j.neulet.2009.10.018

Buehner, M., Mangels, M., Krumm, S., \& Ziegler, M. (2005). Are Working Memory and Attention Related Constructs? Journal of Individual Differences, 26(3), 121-131. https://doi.org/10.1027/1614-0001.26.3.121

Bueichekú, E., Miró-Padilla, A., Palomar-García, M.-T., Ventura-Campos, N., Parcet, M.-A., Barrós-Loscertales, A., \& Ávila, C. (2016). Reduced posterior parietal cortex activation after training on a visual search task. NeuroImage, 135, 204-213. https://doi.org/10.1016/j.neuroimage.2016.04.059

Bueichekú, E., Ventura-Campos, N., Palomar-García, M.-A., Miró-Padilla, A., Parcet, M.-A., \& Ávila, C. (2015). Functional connectivity between superior parietal lobule and primary visual cortex "at Rest" predicts visual search efficiency. Brain Connectivity, 5(8), 517-526. https://doi.org/10.1089/brain.2015.0352

Buschkuehl, M., Hernandez-Garcia, L., Jaeggi, S. M., Bernard, J. a, \& Jonides, J. (2014). Neural effects of short-term training on working memory. Cognitive, Affective \& Behavioral Neuroscience, 14(1), 147-160. https://doi.org/10.3758/s13415-0130244-9

Buschkuehl, M., Jaeggi, S. M., \& Jonides, J. (2012). Neuronal effects following working memory training. Developmental Cognitive Neuroscience, 2, Supplem, S167-S179. https://doi.org/10.1016/j.den.2011.10.001

Bush, G., Luu, P., \& Posner, M. (2000). Cognitive and emotional influences in anterior cingulate cortex. Trends Cogn. Sci., 4(6), 215-222. https://doi.org/10.1016/S13646613(00)01483-2

Caeyenberghs, K., Metzler-Baddeley, C., Foley, S., \& Jones, D. K. (2016). Dynamics of the Human Structural Connectome Underlying Working Memory Training. The Journal of Neuroscience, 36(14), 4056 LP-4066.

Case, R., Kurland, D. M., \& Goldberg, J. (1982). Operational efficiency and the growth of short-term memory span. Journal of Experimental Child Psychology, 33(3), 386404. https://doi.org/10.1016/0022-0965(82)90054-6 
Caspers, S., Eickhoff, S. B., Rick, T., von Kapri, A., Kuhlen, T., Huang, R., ... Zilles, K. (2011). Probabilistic fibre tract analysis of cytoarchitectonically defined human inferior parietal lobule areas reveals similarities to macaques. NeuroImage, 58(2), 362-380. https://doi.org/10.1016/J.NEUROIMAGE.2011.06.027

Cauda, F., D’Agata, F., Sacco, K., Duca, S., Geminiani, G., \& Vercelli, A. (2011). Functional connectivity of the insula in the resting brain. NeuroImage, 55(1), 8-23. https://doi.org/10.1016/J.NEUROIMAGE.2010.11.049

Chang, L., Løhaugen, G. C., Andres, T., Jiang, C. S., Douet, V., Tanizaki, N., ... Ernst, T. M. (2017). Adaptive working memory training improved brain function in human immunodeficiency virus-seropositive patients. Annals of Neurology, 81(1), 17-34. https://doi.org/10.1002/ana.24805

Chang, Y. (2014). Reorganization and plastic changes of the human brain associated with skill learning and expertise. Frontiers in Human Neuroscience, 8, 35. Retrieved from https://www.frontiersin.org/article/10.3389/fnhum.2014.00035

Chen, S. H. A., \& Desmond, J. E. (2005). Temporal dynamics of cerebro-cerebellar network recruitment during a cognitive task. Neuropsychologia , 43(9), 1227-1237. https://doi.org/10.1016/J.NEUROPSYCHOLOGIA.2004.12.015

Chooi, W.-T., \& Thompson, L. A. (2012). Working memory training does not improve intelligence in healthy young adults. Intelligence, 40(6), 531-542. https://doi.org/10.1016/J.INTELL.2012.07.004

Clark, C. M., Lawlor-Savage, L., \& Goghari, V. M. (2017a). Functional Brain Activation Associated with Working Memory Training and Transfer. Behavioural Brain Research, 334(May), 34-49. https://doi.org/10.1016/j.bbr.2017.07.030

Clark, C. M., Lawlor-Savage, L., \& Goghari, V. M. (2017b). Working memory training in healthy young adults: Support for the null from a randomized comparison to active and passive control groups, 1-25. https://doi.org/10.6084/m9.figshare.4551517

Cohen, J. D., Braver, T. S., \& Brown, J. W. (2002, April 1). Computational perspectives on dopamine function in prefrontal cortex. Current Opinion in Neurobiology. Elsevier Current Trends. https://doi.org/10.1016/S0959-4388(02)00314-8

Collette, F., Hogge, M., Salmon, E., \& Van der Linden, M. (2006). Exploration of the neural substrates of executive functioning by functional neuroimaging. Neuroscience, 139(1), 209-221. https://doi.org/10.1016/j.neuroscience.2005.05.035

Collette, F., \& Van Der Linden, M. (2002). Brain imaging of the central executive component of working memory. Neuroscience and Biobehavioral Reviews, 26(2), 105-125. https://doi.org/10.1016/S0149-7634(01)00063-X

Colom, R., Martínez, K., Burgaleta, M., Román, F. J., García-García, D., Gunter, J. L., ... Thompson, P. M. (2016). Gray matter volumetric changes with a challenging adaptive cognitive training program based on the dual n-back task. Personality and Individual Differences, 98, 127-132. https://doi.org/10.1016/J.PAID.2016.03.087

Constantinidis, C., \& Klingberg, T. (2016). The neuroscience of working memory capacity and training. Nature Reviews Neuroscience, 17(7), 438-449. https://doi.org/10.1038/nrn.2016.43

Conway, A. R. A., Kane, M. J., \& Engle, R. W. (2003). Working memory capacity and its relation to general intelligence. Trends in Cognitive Sciences, 7(12), 547-552. https://doi.org/10.1016/j.tics.2003.10.005

Correia, S. (2011). Paced Auditory Serial Attention Test. In J. S. Kreutzer, J. DeLuca, \& B. Caplan (Eds.), Encyclopedia of Clinical Neuropsychology (pp. 1840-1845). New York, NY: Springer New York. https://doi.org/10.1007/978-0-387-79948-3_1319

Cowan, N. (1988). Evolving Concenptions of Memory Storage, Selective Attention, and 
their Mutual Constraits Within the Human Information-Processing System. Psychological Bulletin, 104(2), 163-191.

Cowan, N. (1999). An Embedded-Processes Model of Working Memory. In A. Miyake \& P. Shah (Eds.), Models of Working Memory: Mechanisms of Active Maintenance and Executive Control (pp. 62-101). Cambridge: Cambridge University Press. https://doi.org/DOI: 10.1017/CBO9781139174909.006

Cowan, N. (2001). The magical number 4 in short-term memory: A reconsideration of mental storage capacity. Behavioral and Brain Sciences, 24(1), S0140525X01003922. https://doi.org/10.1017/S0140525X01003922

Cowan, N. (2009). What are the differences between long-term, short-term, and working memory? NIH Public Access, 6123(07), 323-338. https://doi.org/10.1016/S00796123(07)00020-9. What

Cowan, N. (2010). Multiple Concurrent Thoughts: The Meaning and Developmental Neuropsychology of Working Memory. Developmental Neuropsychology, 35(5), 447-474. https://doi.org/10.1080/87565641.2010.494985

Crossman, A. R., \& Neary, D. (2007). Neuroanatomía : texto y atlas en color. Elsevier Masson (3th editio, Vol. 3 edición). Barcelona: Elsevier Masson. Retrieved from http://cataleg.uji.es/record=b1237700 S1*cat

Curtis, C. E., \& D'Esposito, M. (2003). Persistent activity in the prefrontal cortex during working memory. Trends in Cognitive Sciences, 7(9), 415-423. https://doi.org/10.1016/S1364-6613(03)00197-9

D’Ardenne, K., Eshel, N., Luka, J., Lenartowicz, A., Nystrom, L. E., \& Cohen, J. D. (2012). Role of prefrontal cortex and the midbrain dopamine system in working memory updating. Proceedings of the National Academy of Sciences, 109(49), 19900. Retrieved from http://www.pnas.org/content/109/49/19900.abstract

D'Esposito, M. (2007). From cognitive to neural models of working memory. Philosophical Transactions of the Royal Society B: Biological Sciences, 362(1481), 761-772. https://doi.org/10.1098/rstb.2007.2086

D’Esposito, M., Detre, J. A., Alsop, D. C., Shin, R. K., Atlas, S., \& Grossman, M. (1995). The neural basis of the central executive system of working memory. Nature, 378(6554), 279-281. https://doi.org/10.1038/378279a0

D'Esposito, M., \& Postle, B. R. (2015). The Cognitive Neuroscience of Working Memory. Annu Rev Psychol., (66), 115-142. https://doi.org/10.1146/annurevpsych-010814-015031.THE

Dahlin, E., Neely, A. S., Larsson, A., Backman, L., \& Nyberg, L. (2008). Transfer of Learning After Updating Training Mediated by the Striatum. Science, 320(5882), 1510-1512. https://doi.org/10.1126/science.1155466

Dahlin, E., Neely, A. S., Larsson, A., Bäckman, L., \& Nyberg, L. (2008). Transfer of learning after updating training mediated by the striatum. Science (New York, N.Y.), 320(5882), 1510-1512. https://doi.org/10.1126/science.1155466

Daneman, M., \& Carpenter, P. A. (1980). Individual differences in working memory and reading. Journal of Verbal Learning and Verbal Behavior, 19(4), 450-466. https://doi.org/10.1016/S0022-5371(80)90312-6

Daneman, M., \& Merikle, P. M. (1996). Working memory and language comprehension: A meta-analysis. Psychonomic Bulletin \& Review, 3(4), 422-433. https://doi.org/10.3758/BF03214546

Debaere, F., Wenderoth, N., Sunaert, S., Van Hecke, P., \& Swinnen, S. . (2004). Cerebellar and premotor function in bimanual coordination: parametric neural responses to spatiotemporal complexity and cycling frequency. NeuroImage, 21(4), 1416-1427. https://doi.org/10.1016/j.neuroimage.2003.12.011 
Dehaene, S., Piazza, M., Pinel, P., \& Cohen, L. (2003). Three parietal circuits for number processing. Cognitive Neuropsychology, 20(3-6), 487-506. https://doi.org/10.1080/02643290244000239

Dehn, M. J. (2008). Working Memory and Academic Learning: Assessment and Intervention. Retrieved

from https://books.google.com/books?hl=en\&lr=\&id=C2uZNzgo29QC\&pgis=1

Denis, Michel; Logie, Robert; Cornoldo, C. (2012). The processing of visuo-spatial information: Neuropsychological and neuroimaging investigations. In magery, Language and Visuo-Spatial Thinking (Psychology, pp. 81-102). Hove, US.

DeYoung, C. G., Shamosh, N. A., Green, A. E., Braver, T. S., \& Gray, J. R. (2009). Intellect as Distinct From Openness: Differences Revealed by fMRI of Working Memory. Journal of Personality and Social Psychology, 97(5), 883-892. https://doi.org/10.1037/a0016615

Dobbs, a R., \& Rule, B. G. (1989). Adult age differences in working memory. Psychology and Aging, 4(4), 500-503. https://doi.org/10.1037//0882-7974.4.4.500

Dosenbach, N. U. F., Fair, D. A., Miezin, F. M., Cohen, A. L., Wenger, K. K., Dosenbach, R. A. T., ... Petersen, S. E. (2007). Distinct brain networks for adaptive and stable task control in humans. Proceedings of the National Academy of Sciences, 104(26), 11073-11078. https://doi.org/10.1073/pnas.0704320104

Dougherty, M. R., Hamovitz, T., \& Tidwell, J. W. (2016). Reevaluating the effectiveness of n-back training on transfer through the Bayesian lens: Support for the null. Psychonomic Bulletin \& Review, 23(1), 306-316. https://doi.org/10.3758/s13423015-0865-9

Draganski, B., Gaser, C., Busch, V., Schuierer, G., Bogdahn, U., \& May, A. (2004). Changes in grey matter induced by training. Nature, 427, 311. Retrieved from http://dx.doi.org/10.1038/427311a

Draganski, B., Gaser, C., Kempermann, G., Kuhn, H. G., Winkler, J., Büchel, C., \& May, A. (2006). Temporal and Spatial Dynamics of Brain Structure Changes during Extensive Learning. The Journal of Neuroscience, 26(23), 6314. Retrieved from http://www.jneurosci.org/content/26/23/6314.abstract

Durstewitz, D., Seamans, J. K., \& Sejnowski, T. J. (2000a). Dopamine-Mediated Stabilization of Delay-Period Activity in a Network Model of Prefrontal Cortex. Journal of Neurophysiology, 83(3), 1733-1750. https://doi.org/10.1152/jn.2000.83.3.1733

Durstewitz, D., Seamans, J. K., \& Sejnowski, T. J. (2000b). Neurocomputational models of working memory. Nature Neuroscience, 3, 1184. Retrieved from http://dx.doi.org/10.1038/81460

Edin, F., Klingberg, T., Johansson, P., McNab, F., Tegnér, J., \& Compte, A. (2009). Mechanism for top-down control of working memory capacity. Proceedings of the National Academy of Sciences of the United States of America, 106(16), 6802-6807. https://doi.org/10.1073/pnas.0901894106

Engvig, A., Fjell, A. M., Westlye, L. T., Moberget, T., Sundseth, Ø., Larsen, V. A., \& Walhovd, K. B. (2010). Effects of memory training on cortical thickness in the elderly. NeuroImage, 52(4), 1667-1676. https://doi.org/10.1016/J.NEUROIMAGE.2010.05.041

Eriksson, J., Vogel, E. K., Lansner, A., Bergstr??m, F., Nyberg, L., Bergström, F., \& Nyberg, L. (2015). Neurocognitive Architecture of Working Memory. Neuron, 88(1), 33-46. https://doi.org/10.1016/j.neuron.2015.09.020

Farah, M. J., Hammond, K. M., Levine, D. ., \& Calvanio, R. (1988). Visual and spatial mentalimagery: Dissociable systems of representation. Cognitive Psychology, 20, 
439-462. https://doi.org/10.1016/0010-0285(88)90012-6

Forn, C., Belenguer, A., Belloch, V., Sanjuan, A., Parcet, M. A., \& Avila, C. (2011). Anatomical and functional differences between the Paced Auditory Serial Addition Test and the Symbol Digit Modalities Test. Journal of Clinical and Experimental Neuropsychology, 33(1), 42-50. https://doi.org/10.1080/13803395.2010.481620

Frank, M. J., Loughry, B., \& O'Reilly, R. C. (2001). Interactions between frontal cortex and basal ganglia in working memory: A computational model. Cognitive, Affective, \& Behavioral Neuroscience, 1(2), 137-160. https://doi.org/10.3758/CABN.1.2.137

Funahashi, S. (2017). Working Memory in the Prefrontal Cortex. Brain Sciences, 7(5), 49. https://doi.org/10.3390/brainsci7050049

Funahashi, S., \& Andreau, J. M. (2013). Prefrontal cortex and neural mechanisms of executive function. Journal of Physiology-Paris, 107(6), 471-482. https://doi.org/10.1016/j.jphysparis.2013.05.001

Fuster, J. M. (2008). The Prefrontal Cortex. (Academic Press, Ed.) (4th ed.). New York, NY, USA.

Fuster, J. M. (2009). Cortex and Memory: Emergence of a New Paradigm. Journal of Cognitive Neuroscience, 21(11), 2047-2072. https://doi.org/10.1162/jocn.2009.21280

Garavan, H., Kelley, D., Rosen, A., Rao, S. M., \& Stein, E. A. (2000). Practice-related functional activation changes in a working memory task. Microscopy Research and Technique, 51(1), 54-63. $\quad$ https://doi.org/10.1002/10970029(20001001)51:1<54::AID-JEMT6>3.0.CO;2-J

Gaser, C., \& Schlaug, G. (2003). Brain Structures Differ between Musicians and NonMusicians. The Journal of Neuroscience, 23(27), 9240. Retrieved from http://www.jneurosci.org/content/23/27/9240.abstract

Gaser C Schlaug G. (2003). Gray Matter Differences between Musicians and Nonmusicians. Annals of the New York Academy of Sciences, 999(1), 514-517. https://doi.org/10.1196/annals.1284.062

Gathercole, S. E., \& Baddeley, A. D. (1993). Working Memory and Language. Lawrence Erlbaum. Retrieved from https://books.google.es/books?id=lCLnqNRRc1AC

Gibson, E. M., Purger, D., Mount, C. W., Goldstein, A. K., Lin, G. L., Wood, L. S., ... Monje, M. (2014). Neuronal Activity Promotes Oligodendrogenesis and Adaptive Myelination in the Mammalian Brain. Science, 344(6183). Retrieved from http://science.sciencemag.org/content/344/6183/1252304.abstract

Glickstein, M. (1993). Motor skills but not cognitive tasks. Trends in Neurosciences, 16(11), 450-1; discussion 453-4. Retrieved from http://www.ncbi.nlm.nih.gov/pubmed/7507616

Goldman-Rakic, P. S. (1987). Circuitry of primate prefrontal cortex and regulation of behavior by representational memory. In F. Plum (Ed.), Higher Functions of the Brain: The Nervous System; Handbook of Physiology (pp. 373-417). Hoboken, NJ, USA: John Wiley \& Sons, Inc. https://doi.org/10.1002/cphy.cp010509

Goldstein, E. B. (2008). Cognitive Psychology: Connecting Mind, Research, and Everyday Experience. Manual (Third). Belmont, CA Wadsworth/Cengage Learning. https://doi.org/9781133889748

Golestani, N., \& Pallier, C. (2007). Anatomical Correlates of Foreign Speech Sound Production. Cerebral Cortex, 17(4), 929-934. Retrieved from http://dx.doi.org/10.1093/cercor/bhl003

Golestani, N., Paus, T., \& Zatorre, R. J. (2002). Anatomical Correlates of Learning Novel Speech Sounds. Neuron, 35(5), 997-1010. https://doi.org/10.1016/S08966273(02)00862-0 
Golestani, N., \& Zatorre, R. J. (2004). Learning new sounds of speech: reallocation of neural substrates. NeuroImage, 21(2), 494-506. https://doi.org/10.1016/J.NEUROIMAGE.2003.09.071

Grace, A. A. (2000). The tonic/phase model of dopamine system regulation and its implications for understanding alcohol and psychostimulant craving. Addiction, 95(Supp12), S119-S128. https://doi.org/10.1080/09652140050111690

Gronwall, D. M. A. (1977). Paced Auditory Serial-Addition Task: A Measure of Recovery from Concussion. Perceptual and Motor Skills, 44(2), 367-373. https://doi.org/10.2466/pms.1977.44.2.367

Haier, R. J., Siegel, B., Tang, C., Abel, L., \& Buchsbaum, M. S. (1992). Intelligence and changes in regional cerebral glucose metabolic rate following learning. Intelligence, 16(3-4), 415-426. https://doi.org/10.1016/0160-2896(92)90018-M

Hanley, J. R., Young, A. W., \& Pearson, N. A. (1991). IMPAIRMENT OF THE VISUOSPATIAL SKETCH PAD. The Quarterly Journal of Experimental Psychology. A, Human Experimental Psychology, 43(1), 101-125.

Harrison, T. L., Shipstead, Z., Hicks, K. L., Hambrick, D. Z., Redick, T. S., \& Engle, R. W. (2013). Working Memory Training May Increase Working Memory Capacity but Not Fluid Intelligence. Psychological Science, 24(12), 2409-2419. https://doi.org/10.1177/0956797613492984

Hayter, A. L., Langdon, D. W., \& Ramnani, N. (2007). Cerebellar contributions to working memory. NeuroImage, 36(3), 943-954. https://doi.org/10.1016/j.neuroimage.2007.03.011

Hebb, D. O. (1949). Organization of behavior. Wiley: New York.

Hedden, T., \& Yoon, C. (2006). Individual differences in executive processing predict susceptibility to interference in verbal working memory. Neuropsychology, 20(5), 511-528. https://doi.org/10.1037/0894-4105.20.5.511

Hempel, A., Giesel, F. L., Garcia Caraballo, N. M., Amann, M., Meyer, H., Wüstenberg, T., ... Schröder, J. (2004). Plasticity of Cortical Activation Related to Working Memory during Training. American Journal of Psychiatry, (April), 745-747. https://doi.org/10.1176/appi.ajp.161.4.745

Hindin, S. B., \& Zelinski, E. M. (2011). Extended Practice and Aerobic Exercise Interventions Benefit Untrained Cognitive Outcomes in Older Adults: A MetaAnalysis. Journal of the American Geriatrics Society, 60(1), 136-141. https://doi.org/10.1111/j.1532-5415.2011.03761.x

Holmes, G. (1939). The Cerebellum of Man. Brain, 62(1), 1-30. https://doi.org/10.1093/brain/62.1.1

Humphreys, G. F., \& Lambon Ralph, M. A. (2015). Fusion and Fission of Cognitive Functions in the Human Parietal Cortex. Cerebral Cortex, 25(10), 3547-3560. Retrieved from http://dx.doi.org/10.1093/cercor/bhu198

Hwang, K., Velanova, K., \& Luna, B. (2010). Strengthening of Top-Down Frontal Cognitive Control Networks Underlying the Development of Inhibitory Control: A Functional Magnetic Resonance Imaging Effective Connectivity Study. Journal of Neuroscience, 30(46), 15535-15545. https://doi.org/10.1523/JNEUROSCI.282510.2010

Hyde, K. L., Lerch, J., Norton, A., Forgeard, M., Winner, E., Evans, A. C., \& Schlaug, G. (2009). Musical Training Shapes Structural Brain Development. The Journal of Neuroscience, $\quad 29(10), \quad 3019 . \quad$ Retrieved from http://www.jneurosci.org/content/29/10/3019.abstract

Ilg, R., Wohlschläger, A. M., Gaser, C., Liebau, Y., Dauner, R., Wöller, A., ... Mühlau, M. (2008). Gray Matter Increase Induced by Practice Correlates with Task-Specific 
Activation: A Combined Functional and Morphometric Magnetic Resonance Imaging Study. The Journal of Neuroscience, 28(16), 4210. Retrieved from http://www.jneurosci.org/content/28/16/4210.abstract

Jaeggi, S. M., Buschkuehl, M., Jonides, J., \& Perrig, W. J. (2008). Improving fluid intelligence with training on working memory. Proceedings of the National Academy of Sciences of the United States of America, 105(19), 6829-6833. https://doi.org/10.1073/pnas.0801268105

Jaeggi, S. M., Buschkuehl, M., Jonides, J., \& Shah, P. (2011). Short- and long-term benefits of cognitive training. Proceedings of the National Academy of Sciences, 108(25), 10081-10086. https://doi.org/10.1073/pnas.1103228108

Jaeggi, S. M., Buschkuehl, M., Perrig, W. J., \& Meier, B. (2010). The concurrent validity of the $N$-back task as a working memory measure. Memory, 18(4), 394-412. https://doi.org/10.1080/09658211003702171

Jaeggi, S. M., Buschkuehl, M., Shah, P., \& Jonides, J. (2014). The role of individual differences in cognitive training and transfer. Memory \& Cognition, 42, 464-480. https://doi.org/10.3758/s13421-013-0364-z

Jaeggi, S. M., Seewer, R., Nirkko, A. C., Eckstein, D., Schroth, G., Groner, R., \& Gutbrod, K. (2003). Does excessive memory load attenuate activation in the prefrontal cortex? Load-dependent processing in single and dual tasks: functional magnetic resonance imaging study. NeuroImage, 19(2), 210-225. https://doi.org/10.1016/S1053-8119(03)00098-3

Jaeggi, S. M., Studer-Luethi, B., Buschkuehl, M., Su, Y.-F., Jonides, J., \& Perrig, W. J. (2010). The relationship between n-back performance and matrix reasoning implications for training and transfer. Intelligence, 38(6), 625-635. https://doi.org/10.1016/j.intell.2010.09.001

James, W. (1890). The Principles of Psychology. New York: Holt.

Jäncke, L. (2009). The plastic human brain. Restorative Neurology and Neuroscience, 27(5), 521-538. https://doi.org/10.3233/RNN-2009-0519

Jansma, J. M., Ramsey, N. F., Slagter, H. A., \& Kahn, R. S. (2001). Functional Anatomical Correlates of Controlled and Automatic Processing. Journal of Cognitive Neuroscience, 13(6), 730-743. https://doi.org/10.1162/08989290152541403

Johns, P. (2014). Functional neuroanatomy. In Clinical Neuroscience (pp. 27-47). Elsevier. https://doi.org/10.1016/B978-0-443-10321-6.00003-5

Johnstone, T., van Reekum, C. M., Urry, H. L., Kalin, N. H., \& Davidson, R. J. (2007). Failure to Regulate: Counterproductive Recruitment of Top-Down PrefrontalSubcortical Circuitry in Major Depression. Journal of Neuroscience, 27(33), 88778884. https://doi.org/10.1523/JNEUROSCI.2063-07.2007

Jolles, D. D., van Buchem M. A., Crone, E. A., \& Rombouts, S. A. (2011). Functional brain connectivity at rest changes after working memory training. Human Brain Mapping, 34(2), 396-406. https://doi.org/10.1002/hbm.21444

Jonides, J., Smith, E. E., Koeppe, R. A., Awh, E., Minoshima, S., \& Mintun, M. A. (1993). Spatial working memory in humans as revealed by PET. Nature, 363(6430), 623-625. https://doi.org/10.1038/363623a0

Kane, M. J., \& Engle, R. W. (2002). The role of prefrontal cortex in working-memory capacity, executive attention, and general fluid intelligence: An individualdifferences perspective. Psychonomic Bulletin \& Review, 9(4), 637-671. https://doi.org/10.3758/BF03196323

Kane, M. J., Hambrick, D. Z., Tuholski, S. W., Wilhelm, O., Payne, T. W., \& Engle, R. W. (2004). The Generality of Working Memory Capacity: A Latent-Variable 
Approach to Verbal and Visuospatial Memory Span and Reasoning. Journal of Experimental Psychology: General, 133(2), 189-217. https://doi.org/10.1037/00963445.133.2.189

Karbach, J., \& Verhaeghen, P. (2014). Making Working Memory Work: A MetaAnalysis of Executive-Control and Working Memory Training in Older Adults. Psychological Science, 25(11), 2027-2037. https://doi.org/10.1177/0956797614548725

Karr, J. E., Areshenkoff, C. N., Rast, P., \& Garcia-Barrera, M. A. (2014). An empirical comparison of the therapeutic benefits of physical exercise and cognitive training on the executive functions of older adults: A meta-analysis of controlled trials. Neuropsychology, 28(6), 829-845. https://doi.org/10.1037/neu0000101

Katz, B., Au, J., Buschkuehl, M., Abagis, T., Zabel, C., Jaeggi, S. M., \& Jonides, J. (2017). Individual Differences and Long-term Consequences of tDCS-augmented Cognitive Training. Journal of Cognitive Neuroscience, 29(9), 1498-1508. https://doi.org/10.1162/jocn_a_01115

Kelly, A. M. C., \& Garavan, H. (2005). Human functional neuroimaging of brain changes associated with practice. Cerebral Cortex, 15(8), 1089-1102. https://doi.org/10.1093/cercor/bhi005

Kelly, C., \& Castellanos, F. X. (2014). Strengthening Connections: Functional Connectivity and Brain Plasticity. Neuropsychology Review, 24(1), 63-76. https://doi.org/10.1007/s11065-014-9252-y

Kelly, C., Foxe, J. J., \& Garavan, H. (2006). Patterns of Normal Human Brain Plasticity After Practice and Their Implications for Neurorehabilitation. Archives of Physical Medicine and Rehabilitation, 87(12 SUPPL.), 20-29. https://doi.org/10.1016/j.apmr.2006.08.333

Kelly, M. E., Loughrey, D., Lawlor, B. A., Robertson, I. H., Walsh, C., \& Brennan, S. (2014). The impact of cognitive training and mental stimulation on cognitive and everyday functioning of healthy older adults: A systematic review and metaanalysis. Ageing Research Reviews, 15, 28-43. https://doi.org/10.1016/j.arr.2014.02.004

Keren-Happuch, E., Shen-Hsing, A. C., Ringo Ho, M., \& Desmond, J. E. (2012). A metaanalysis of cerebellar contributions to higher cognition from PET and fMRI studies. Human Brain Mapping, 35(2), 593-615. https://doi.org/10.1002/hbm.22194

Kirchner, W. K. (1958). Age differences in short-term retention of rapidly changing information. Journal of Experimental Psychology, 55(4), 352-358. https://doi.org/10.1037/h0043688

Kleim, J. A., Chan, S., Pringle, E., Schallert, K., Procaccio, V., Jimenez, R., \& Cramer, S. C. (2006). BDNF val66met polymorphism is associated with modified experience-dependent plasticity in human motor cortex. Nature Neuroscience, 9, 735. Retrieved from http://dx.doi.org/10.1038/nn1699

Klingberg, T. (2010). Training and plasticity of working memory. Trends in Cognitive Sciences, 14(7), 317-324. https://doi.org/10.1016/j.tics.2010.05.002

Koenigs, M., Barbey, A. K., Postle, B. R., \& Grafman, J. (2009). Superior parietal cortex is critical for the manipulation of information in working memory. The Journal of Neuroscience : The Official Journal of the Society for Neuroscience, 29(47), 1498014986. https://doi.org/10.1523/JNEUROSCI.3706-09.2009

Kundu, B., Sutterer, D. W., Emrich, S. M., \& Postle, B. R. (2013). Strengthened effective connectivity underlies transfer of working memory training to tests of short-term memory and attention. The Journal of Neuroscience : The Official Journal of the Society for Neuroscience, 33(20), 8705-8715. 
https://doi.org/10.1523/JNEUROSCI.5565-12.2013

Küper, K., \& Karbach, J. (2016). Increased training complexity reduces the effectiveness of brief working memory training: evidence from short-term single and dual $\mathrm{n}$-back training interventions. Journal of Cognitive Psychology, 28(2), 199-208. https://doi.org/10.1080/20445911.2015.1118106

Lara, A. H., \& Wallis, J. D. (2015). The role of prefrontal cortex in working memory: a mini review. Frontiers in Systems Neuroscience, 9(December), 1-7. https://doi.org/10.3389/fnsys.2015.00173

Lee, T. G., \& D'Esposito, M. (2012). The Dynamic Nature of Top-Down Signals Originating from Prefrontal Cortex: A Combined fMRI-TMS Study. Journal of Neuroscience, 32(44), 15458-15466. https://doi.org/10.1523/JNEUROSCI.062712.2012

Lewis, S., Dove, A., Robbins, T., Barker, R., \& Owen, A. (2004). Striatal contributions to working memory: a functional magnetic resonance imaging study in humans. European Journal of Neuroscience, 19(3), 755-760. https://doi.org/10.1111/j.14609568.2004.03108.x

Li, S.-C., Schmiedek, F., Huxhold, O., Röcke, C., Smith, J., \& Lindenberger, U. (2008). Working memory plasticity in old age: Practice gain, transfer, and maintenance. Psychology and Aging, 23(4), 731-742. https://doi.org/10.1037/a0014343

Libby, L. A., Hannula, D. E., \& Ranganath, C. (2014). Medial Temporal Lobe Coding of Item and Spatial Information during Relational Binding in Working Memory. The Journal of Neuroscience, 34(43), 14233. Retrieved from http://www.jneurosci.org/content/34/43/14233.abstract

Logan, G. D. (1988). Toward an instance theory of automatization. Psychological Review, 95(4), 492-527. https://doi.org/10.1037/0033-295X.95.4.492

Logie, R. H. (1996). The Seven Ages of Working Memory. In Working Memory and Human Cognition (pp. 31-60). Oxford University Press. https://doi.org/10.1093/acprof:oso/9780195100990.003.0002

Logie, R. H. (2011). The Functional Organization and Capacity Limits of Working Memory. Current Directions in Psychological Science, 20(4), 240-245. https://doi.org/10.1177/0963721411415340

López, M. (2011). Memoria de trabajo y aprendizaje:aportes de la neuropsicologia. Cuad. Neuropsicol ${ }^{\circ}$, 5(1), 25-47. Retrieved from www.cnps.cl

Maguire, E. A., Gadian, D. G., Johnsrude, I. S., Good, C. D., Ashburner, J., Frackowiak, R. S. J., \& Frith, C. D. (2000). Navigation-related structural change in the hippocampi of taxi drivers. Proceedings of the National Academy of Sciences, 97(8), 4398. Retrieved from http://www.pnas.org/content/97/8/4398.abstract

Mai, J. K., \& Forutan, F. (2012). Thalamus. In The Human Nervous System (pp. 618677). Elsevier. https://doi.org/10.1016/B978-0-12-374236-0.10019-7

Marien, P., Engelborghs, S., Fabbro, F., \& De Deyn, P. P. (2001). The Lateralized Linguistic Cerebellum: A Review and a New Hypothesis. Brain and Language, 79(3), 580-600. https://doi.org/10.1006/BRLN.2001.2569

Mars, R. B., Jbabdi, S., Sallet, J., O\&\#039;Reilly, J. X., Croxson, P. L., Olivier, E., ... Rushworth, M. F. S. (2011). Diffusion-Weighted Imaging Tractography-Based Parcellation of the Human Parietal Cortex and Comparison with Human and Macaque Resting-State Functional Connectivity. The Journal of Neuroscience, 31(11), 4087. Retrieved from http://www.jneurosci.org/content/31/11/4087.abstract

Mårtensson, J., Eriksson, J., Bodammer, N. C., Lindgren, M., Johansson, M., Nyberg, L., \& Lövdén, M. (2012). Growth of language-related brain areas after foreign language learning.

NeuroImage,

63(1),

240-244. 
https://doi.org/10.1016/J.NEUROIMAGE.2012.06.043

McNab, F., \& Klingberg, T. (2007). Prefrontal cortex and basal ganglia control access to working memory. Nature Neuroscience, 11, 103. Retrieved from http://dx.doi.org/10.1038/nn2024

McNab, F., Varrone, A., Farde, L., Jucaite, A., Bystritsky, P., Forssberg, H., \& Klingberg, T. (2009). Changes in Cortical Dopamine D1 Receptor Binding Associated with Cognitive Training. Science, 323(5915), 800-802. https://doi.org/10.1126/science.1166102

Melby-Lervåg, M., \& Hulme, C. (2013). Is working memory training effective? A metaanalytic review. Developmental Psychology, 49(2), 270-291. https://doi.org/10.1037/a0028228

Melby-Lervåg, M., \& Hulme, C. (2016). There is no convincing evidence that working memory training is effective: A reply to Au et al. (2014) and Karbach and Verhaeghen (2014). Psychonomic Bulletin \& Review, 23(1), 324-330. https://doi.org/10.3758/s13423-015-0862-z

Melby-Lervåg, M., Redick, T., \& Hulme, C. (2016). Working Memory Training Does Not Improve Performance on Measures of Intelligence or Other Measures of "Far Transfer": Evidence from a Meta-Analytic Review. Perspectives on Psychological Science, 11(4). https://doi.org/10.3837/tiis.0000.00.000

Menon, V., \& Uddin, L. Q. (2010). Saliency, switching, attention and control: a network model of insula function. Brain Structure and Function, 214(5-6), 655-667. https://doi.org/10.1007/s00429-010-0262-0

Miller, G. A. (1956). The magical number seven plus or minus two: some limits on our capacity for processing information. Psychological Review, 63(2), 81-97. Retrieved from http://www.ncbi.nlm.nih.gov/pubmed/13310704

Miller, K. M., Price, C. C., Okun, M. S., Montijo, H., \& Bowers, D. (2009). Is the NBack Task a Valid Neuropsychological Measure for Assessing Working Memory? Archives of Clinical Neuropsychology, 24(7), 711-717. https://doi.org/10.1093/arclin/acp063

Minear, M., Brasher, F., Guerrero, C. B., Brasher, M., Moore, A., \& Sukeena, J. (2016). A simultaneous examination of two forms of working memory training: Evidence for near transfer only. Memory \& Cognition, 1-24. https://doi.org/10.3758/s13421016-0616-9

Moore, A. B., Li, Z., Tyner, C. E., Hu, X., \& Crosson, B. (2013). Bilateral basal ganglia activity in verbal working memory. Brain and Language, 125(3), 316-323. https://doi.org/10.1016/J.BANDL.2012.05.003

Moors, A. (2016). Automaticity: Componential, Causal, and Mechanistic Explanations. Annual Review of Psychology, 67(1), 263-287. https://doi.org/10.1146/annurevpsych-122414-033550

Morrison, A. B., \& Chein, J. M. (2011). Does working memory training work? The promise and challenges of enhancing cognition by training working memory. Psychonomic Bulletin \& Review, 18(1), 46-60. https://doi.org/10.3758/s13423-0100034-0

Müller, N. G., \& Knight, R. T. (2006). The functional neuroanatomy of working memory: Contributions of human brain lesion studies. Neuroscience, 139(1), 51-58. https://doi.org/10.1016/j.neuroscience.2005.09.018

Münte, T. F., Altenmüller, E., \& Jäncke, L. (2002). The musician's brain as a model of neuroplasticity. Nature Reviews Neuroscience, 3(6), 473-478. https://doi.org/10.1038/nrn843

Nee, D. E., Brown, J. W., Askren, M. K., Berman, M. G., Demiralp, E., Krawitz, A., \& 
Jonides, J. (2013). A Meta-analysis of Executive Components of Working Memory. Cerebral Cortex, 23(2), 264-282. https://doi.org/10.1093/cercor/bhs007

Nelson, S. M., Cohen, A. L., Power, J. D., Wig, G. S., Miezin, F. M., Wheeler, M. E., ... Petersen, S. E. (2010). A Parcellation Scheme for Human Left Lateral Parietal Cortex. Neuron, 67(1), 156-170. https://doi.org/10.1016/j.neuron.2010.05.025

Neubauer, A. C., \& Fink, A. (2009). Intelligence and neural efficiency: Measures of brain activation versus measures of functional connectivity in the brain. Intelligence, 37(2), 223-229. https://doi.org/10.1016/j.intell.2008.10.008

Nyberg, L., \& Eriksson, J. (2016). Working Memory: Maintenance, Updating, and the Realization of Intentions. Cold Spring Harbor Perspectives in Biology, 8(2), a021816. https://doi.org/10.1101/cshperspect.a021816

O'Halloran, C. J., Kinsella, G. J., \& Storey, E. (2012). The cerebellum and neuropsychological functioning: A critical review. Journal of Clinical and Experimental Neuropsychology, 34(1), 35-56. https://doi.org/10.1080/13803395.2011.614599

O'Reilly, R. C., \& Frank, M. J. (2006). Making Working Memory Work: A Computational Model of Learning in the Prefrontal Cortex and Basal Ganglia. Neural Computation, 18(2), 283-328. https://doi.org/10.1162/089976606775093909

Oberauer, K., Su, H., Wilhelm, O., \& Wittman, W. W. (2003). The multiple faces of working memory: Storage , processing , supervision , and coordination, 31, 167193.

Oberauer, K., Süß, H.-M., Schulze, R., Wilhelm, O., \& Wittmann, W. W. (2000). Working memory capacity - facets of a cognitive ability construct. Personality and Individual Differences, 29(6), 1017-1045. https://doi.org/10.1016/S01918869(99)00251-2

Olesen, P. J., Westerberg, H., \& Klingberg, T. (2004). Increased prefrontal and parietal activity after training of working memory. Nature Neuroscience, 7(1), 75-79. https://doi.org/10.1038/nn1165

Olive, T. (2004). Working Memory in Writing: Empirical Evidence From the Dual-Task Technique. European Psychologist, 9(1), 32-42. https://doi.org/10.1027/10169040.9.1.32

Owen, A. M., McMillan, K. M., Laird, A. R., \& Bullmore, E. (2005). N-back working memory paradigm: a meta-analysis of normative functional neuroimaging studies. Human Brain Mapping, 25(1), 46-59. https://doi.org/10.1002/hbm.20131

Owens, M. M., Duda, B., Sweet, L. H., \& MacKillop, J. (2018). Distinct functional and structural neural underpinnings of working memory. NeuroImage, 174(December 2017), 463-471. https://doi.org/10.1016/j.neuroimage.2018.03.022

Palomar-García, M. Á., Zatorre, R. J., Ventura-Campos, N., Bueichekú, E., \& Ávila, C. (2017). Modulation of Functional Connectivity in Auditory-Motor Networks in Musicians Compared with Nonmusicians. Cerebral Cortex, 27(5), 2768-2778. https://doi.org/10.1093/cercor/bhw120

Pascual-Leone, A., Amedi, A., Fregni, F., \& Merabet, L. B. (2005). THE PLASTIC HUMAN BRAIN CORTEX. Annual Review of Neuroscience, 28(1), 377-401. https://doi.org/10.1146/annurev.neuro.27.070203.144216

Peterburs, J., Bellebaum, C., Koch, B., Schwarz, M., \& Daum, I. (2010). Working Memory and Verbal Fluency Deficits Following Cerebellar Lesions: Relation to Interindividual Differences in Patient Variables. The Cerebellum, 9(3), 375-383. https://doi.org/10.1007/s12311-010-0171-z

Petrides, M. (2000). The role of the mid-dorsolateral prefrontal cortex in working 
memory. Experimental Brain Research, 133(1), 44-54. https://doi.org/10.1007/s002210000399

Petrides, M. (2005). Lateral prefrontal cortex: architectonic and functional organization. Philosophical Transactions of the Royal Society B: Biological Sciences, 360(1456), 781-795. https://doi.org/10.1098/rstb.2005.1631

Poldrack, R. A. (2000). Imaging Brain Plasticity: Conceptual and Methodological Issues- A Theoretical Review. NeuroImage, 12(1), 1-13. https://doi.org/10.1006/NIMG.2000.0596

Poldrack, R. A. (2015). Developmental Cognitive Neuroscience Is " efficiency " a useful concept in cognitive neuroscience ? Developmental Cognitive Neuroscience, 11, 1217. https://doi.org/10.1016/j.den.2014.06.001

Postle, B. R. (2006). Working memory as an emergent property of the mind and brain. Neuroscience, 139(1), 23-38. https://doi.org/10.1016/j.neuroscience.2005.06.005

Postle, B. R., Brush, L. N., \& Nick, A. M. (2004). Prefrontal cortex and the mediation of proactive interference in working memory. Cognitive, Affective \& Behavioral Neuroscience, 4(4), 600-608. https://doi.org/10.1002/nbm.3066

Pribram, Karl H.; Miller, George A.; Galanter, E. (1960). Plans and the structure of behavior. New York: Holt: Rinehart and Winston.

Ravizza, S. M., McCormick, C. A., Schlerf, J. E., Justus, T., Ivry, R. B., \& Fiez, J. A. (2006). Cerebellar damage produces selective deficits in verbal working memory. Brain, 129(2), 306-320. Retrieved from http://dx.doi.org/10.1093/brain/awh685

Redick, T.S., \& Lindsey, D. R. B. (2013). Complex span and n-back measures of working memory: A meta-analysis. Psychonomic Bulletin \& Review, 20(6), 1102-1113. https://doi.org/10.3758/s13423-013-0453-9

Redick, T. S., Shipstead, Z., Harrison, T. L., Hicks, K. L., Fried, D. E., Hambrick, D. Z., ... Engle, R. W. (2013). No Evidence of Intelligence Improvement After Working Memory Training: A Randomized, Placebo-Controlled Study. Journal of Experimental Psychology: General, 142(2), 359-379. https://doi.org/10.1037/a0029082

Reuter-Lorenz, P. A., Jonides, J., Smith, E. E., Hartley, A., Miller, A., Marshuetz, C., \& Koeppe, R. A. (2000). Age Differences in the Frontal Lateralization of Verbal and Spatial Working Memory Revealed by PET. Journal of Cognitive Neuroscience, 12(1), 174-187. https://doi.org/10.1162/089892900561814

Román, F. J., Lewis, L. B., Chen, C. H., Karama, S., Burgaleta, M., Martínez, K., ... Colom, R. (2016). Gray matter responsiveness to adaptive working memory training: a surface-based morphometry study. Brain Structure and Function, 221(9), 4369-4382. https://doi.org/10.1007/s00429-015-1168-7

Rottschy, C., Langner, R., Dogan, I., Reetz, K., Laird, A. R., Schulz, J. B., ... Eickhoff, S. B. (2012). Modelling neural correlates of working memory: A coordinate-based meta-analysis. $\quad$ NeuroImage, 60(1), 830-846. https://doi.org/10.1016/j.neuroimage.2011.11.050

Rudner, M., Fransson, P., Ingvar, M., Nyberg, L., \& Rönnberg, J. (2007). Neural representation of binding lexical signs and words in the episodic buffer of working memory. Neuropsychologia, 45(10), 2258-2276. https://doi.org/10.1016/j.neuropsychologia.2007.02.017

Salminen, T., Kühn, S., Frensch, P. A., \& Schubert, T. (2016). Transfer after Dual n-Back Training Depends on Striatal Activation Change. The Journal of Neuroscience, 36(39), $10198 . \quad$ Retrieved from http://www.jneurosci.org/content/36/39/10198.abstract

Salminen, T., Strobach, T., \& Schubert, T. (2012). On the impacts of working memory 
training on executive functioning. Frontiers in Human Neuroscience, 6(June), 1-14. https://doi.org/10.3389/fnhum.2012.00166

Schmahmann, J. D. (2004). Disorders of the Cerebellum: Ataxia, Dysmetria of Thought, and the Cerebellar Cognitive Affective Syndrome. The Journal of Neuropsychiatry and Clinical Neurosciences, 16(3), 367-378. https://doi.org/10.1176/jnp.16.3.367

Schmahmann, J. D., Weilburg, J. B., \& Sherman, J. C. (2007). The neuropsychiatry of the cerebellum - insights from the clinic. The Cerebellum, 6(3), 254-267. https://doi.org/10.1080/14734220701490995

Schneiders, J. a, Opitz, B., Krick, C. M., \& Mecklinger, A. (2011). Separating intra-modal and across-modal training effects in visual working memory: an fMRI investigation. Cerebral Cortex (New York, N.Y.: 1991), 21(11), 2555-2564. https://doi.org/10.1093/cercor/bhr037

Schneiders, J., Opitz, B., Tang, H., Deng, Y., Xie, C., Li, H., \& Mecklinger, A. (2012). The impact of auditory working memory training on the fronto-parietal working memory network. Frontiers in Human Neuroscience, 6(June), 173. https://doi.org/10.3389/fnhum.2012.00173

Schwaighofer, M., Fischer, F., \& Bühner, M. (2015). Does Working Memory Training Transfer? A Meta-Analysis Including Training Conditions as Moderators. Educational Psychologist, $\quad$ 50(2), 138-166. https://doi.org/10.1080/00461520.2015.1036274

Schweizer, S., Grahn, J., Hampshire, A., Mobbs, D., \& Dalgleish, T. (2013). Training the emotional brain: Improving affective control through emotional working memory training. Annals of Internal Medicine, 158(6), 5301-5311. https://doi.org/10.1523/JNEUROSCI.2593-12.2013

Shah, P., \& Miyake, A. (1996). The separability of working memory resources for spatial thinking and language processing: An individual differences approach. Journal of Experimental Psychology: General, 125(1), 4-27. https://doi.org/10.1037/00963445.125.1.4

Shiffrin, R. M., \& Schneider, W. (1977). Controlled and automatic human information processing: II. Perceptual learning, automatic attending and a general theory. Psychological Review, 84(2), 127-190. https://doi.org/10.1037/0033295X.84.2.127

Shipstead, Z., Redick, T. S., \& Engle, R. W. (2012). Is working memory training effective? Psychological Bulletin, 138(4), 628-654. https://doi.org/10.1037/a0027473

Simons, D. J., Boot, W. R., Charness, N., Gathercole, S. E., Chabris, C. F., Hambrick, D. Z., \& Stine-Morrow, E. A. L. (2016). Do "Brain-Training" Programs Work? Psychological Science in the Public Interest, 17(3), 103-186. https://doi.org/10.1177/1529100616661983

Sluming, V., Barrick, T., Howard, M., Cezayirli, E., Mayes, A., \& Roberts, N. (2002). Voxel-Based Morphometry Reveals Increased Gray Matter Density in Broca's Area in Male Symphony Orchestra Musicians. NeuroImage, 17(3), 1613-1622. https://doi.org/10.1006/NIMG.2002.1288

Smith, E. E., \& Jonides, J. (1997). Working Memory: A View from Neuroimaging. Cognitive Psychology, 33(1), 5-42. https://doi.org/10.1006/cogp.1997.0658

Smith, E. E., Jonides, J., \& Koeppe, R. A. (1996). Dissociating Verbal and Spatial Working Memory Using PET. Cerebral Cortex, 6(1), 11-20. https://doi.org/10.1093/cercor/6.1.11

Söderqvist, S., Matsson, H., Peyrard-Janvid, M., Kere, J., \& Klingberg, T. (2014). Polymorphisms in the Dopamine Receptor 2 Gene Region Influence Improvements 
during Working Memory Training in Children and Adolescents. Journal of Cognitive Neuroscience, 26(1), 54-62. https://doi.org/10.1162/jocn_a_00478

Söderqvist, S., Nutley, S. B., Peyrard-Janvid, M., Matsson, H., Humphreys, K., Kere, J., \& Klingberg, T. (2012). Dopamine, working memory, and training induced plasticity: Implications for developmental research. Developmental Psychology, 48(3), 836-843. https://doi.org/10.1037/a0026179

Soveri, A., Antfolk, J., Karlsson, L., Salo, B., \& Laine, M. (2017). Working memory training revisited: A multi-level meta-analysis of n-back training studies. Psychonomic Bulletin \& Review. https://doi.org/10.3758/s13423-016-1217-0

Spelke, E., Hirst, W., \& Neisser, U. (1976). Skills of divided attention. Cognition, 4(3), 215-230. https://doi.org/10.1016/0010-0277(76)90018-4

Sprenger, A. M., Atkins, S. M., Bolger, D. J., Harbison, J. I., Novick, J. M., Chrabaszcz, J. S., ... Dougherty, M. R. (2013). Intelligence Training working memory : Limits of transfer. Intelligence, 41(5), 638-663. https://doi.org/10.1016/j.intell.2013.07.013

Stein, M., Federspiel, A., Koenig, T., Wirth, M., Strik, W., Wiest, R., ... Dierks, T. (2012). Structural plasticity in the language system related to increased second language proficiency. Cortex, 48(4), 458-465. https://doi.org/10.1016/J.CORTEX.2010.10.007

Stocco, A., Lebiere, C., \& Anderson, J. R. (2010). Conditional Routing of Information to the Cortex: A Model of the Basal Ganglia's Role in Cognitive Coordination. Psychological Review, 117(2), 541-574. https://doi.org/10.1037/a0019077

Stoodley, C. J. (2012). The Cerebellum and Cognition: Evidence from Functional Imaging Studies. The Cerebellum, 11(2), 352-365. https://doi.org/10.1007/s12311011-0260-7

Stoodley, C. J., \& Schmahmann, J. D. (2009). Functional topography in the human cerebellum: A meta-analysis of neuroimaging studies. NeuroImage, 44(2), 489-501. https://doi.org/10.1016/j.neuroimage.2008.08.039

Stuss, D. T., \& Alexander, M. P. (2000). Executive functions and the frontal lobes: a conceptual view. Psychological Research, 63(3-4), 289-298. Retrieved from http://www.ncbi.nlm.nih.gov/pubmed/11004882

Sweet, L. H. (2011). N-Back Paradigm. In J. S. Kreutzer, J. DeLuca, \& B. Caplan (Eds.), Encyclopedia of Clinical Neuropsychology (pp. 1718-1719). New York, NY: Springer New York. https://doi.org/10.1007/978-0-387-79948-3_1315

Takeuchi, H., Taki, Y., \& Kawashima, R. (2010). Effects of working memory training on cognitive functions and neural systems. Reviews in the Neurosciences, 21(6), 427449. Retrieved from http://europepmc.org/abstract/MED/21438192

Takeuchi, H., Taki, Y., Nouchi, R., Hashizume, H., Sekiguchi, A., Kotozaki, Y., ... Kawashima, R. (2013). Effects of working memory training on functional connectivity and cerebral blood flow during rest. Cortex, 49(8), 2106-2125. https://doi.org/10.1016/j.cortex.2012.09.007

Takeuchi, H., Taki, Y., Nouchi, R., Hashizume, H., Sekiguchi, A., Kotozaki, Y., ... Kawashima, R. (2015). Working memory training impacts the mean diffusivity in the dopaminergic system. Brain Structure and Function, 220(6), 3101-3111. https://doi.org/10.1007/s00429-014-0845-2

Takeuchi, H., Taki, Y., Sassa, Y., Hashizume, H., Sekiguchi, A., Fukushima, A., \& Kawashima, R. (2011). Working memory training using mental calculation impacts regional gray matter of the frontal and parietal regions. PLoS ONE, 6(8). https://doi.org/10.1371/journal.pone.0023175

Thomas, C., \& Baker, C. I. (2013). Teaching an adult brain new tricks: A critical review 
of evidence for training-dependent structural plasticity in humans. NeuroImage, 73, 225-236. https://doi.org/10.1016/j.neuroimage.2012.03.069

Thompson, T. W., Waskom, M. L., \& Gabrieli, J. D. E. (2016). Intensive Working Memory Training Produces Functional Changes in Large-scale Frontoparietal Networks. Journal of Cognitive Neuroscience, 28(4), 575-588. https://doi.org/10.1162/jocn_a_00916

Thompson, T. W., Waskom, M. L., Garel, K. L. A., Cardenas-Iniguez, C., Reynolds, G. O., Winter, R., ... Gabrieli, J. D. E. (2013). Failure of Working Memory Training to Enhance Cognition or Intelligence. PLoS ONE, 8(5). https://doi.org/10.1371/journal.pone.0063614

Tombaugh, T. N. (2006). A comprehensive review of the Paced Auditory Serial Addition Test ( PASAT ), 21, 53-76. https://doi.org/10.1016/j.acn.2005.07.006

Tomita, H., Ohbayashi, M., Nakahara, K., Hasegawa, I., \& Miyashita, Y. (1999). Topdown signal from prefrontal cortex in executive control of memory retrieval. Nature, 401(6754), 699-703. https://doi.org/10.1038/44372

Tomlinson, S. P., Davis, N. J., Morgan, H. M., \& Bracewell, R. M. (2014). Cerebellar Contributions to Verbal Working Memory. The Cerebellum, 13(3), 354-361. https://doi.org/10.1007/s12311-013-0542-3

Tops, M., \& Boksem, M. A. S. (2011). A potential role of the inferior frontal gyrus and anterior insula in cognitive control, brain rhythms, and event-related potentials. Frontiers in Psychology, 2(NOV), 1-14. https://doi.org/10.3389/fpsyg.2011.00330

Turner, M. L., \& Engle, R. W. (1989). Is working memory capacity task dependent? Journal of Memory and Language, 28(2), 127-154. https://doi.org/10.1016/0749596X(89)90040-5

Tzourio-Mazoyer, N., Landeau, B., Papathanassiou, D., Crivello, F., Etard, O., Delcroix, N., ... Joliot, M. (2002). Automated Anatomical Labeling of Activations in SPM Using a Macroscopic Anatomical Parcellation of the MNI MRI Single-Subject Brain. NeuroImage, 15(1), 273-289. https://doi.org/10.1006/nimg.2001.0978

Uddin, L. Q., Supekar, K., Amin, H., Rykhlevskaia, E., Nguyen, D. A., Greicius, M. D., \& Menon, V. (2010). Dissociable Connectivity within Human Angular Gyrus and Intraparietal Sulcus: Evidence from Functional and Structural Connectivity. Cerebral Cortex, 20(11), 2636-2646. Retrieved from http://dx.doi.org/10.1093/cercor/bhq011

Ungerleider, L. (2002). Imaging Brain Plasticity during Motor Skill Learning. Neurobiology of Learning and Memory, 78(3), 553-564. https://doi.org/10.1006/nlme.2002.4091

Unsworth, N., \& Engle, R. W. (2007). On the division of short-term and working memory: An examination of simple and complex span and their relation to higher order abilities. Psychological Bulletin, 133(6), 1038-1066. https://doi.org/10.1037/0033-2909.133.6.1038

Vallar, G., \& Papagno, C. (2002). Neuropsychological impairments of short-term memory. Handbook of Memory Disorders. Chichester: Wiley.: John Wiley \& Sons.

Vartanian, O., Jobidon, M.-E., Bouak, F., Nakashima, A., Smith, I., Lam, Q., \& Cheung, B. (2013). Working memory training is associated with lower prefrontal cortex activation in a divergent thinking task. Neuroscience, 236, 186-194. https://doi.org/10.1016/j.neuroscience.2012.12.060

Ventura-Campos, N., Sanjuán, A., González, J., Palomar-García, M.-Á., RodríguezPujadas, A., Sebastián-Gallés, N., ... Ávila, C. (2013). Spontaneous brain activity predicts learning ability of foreign sounds. The Journal of Neuroscience: The Official Journal of the Society for Neuroscience, 33(22), 9295-9305. 
https://doi.org/10.1523/JNEUROSCI.4655-12.2013

von Bastian, C. C., \& Oberauer, K. (2014). Effects and mechanisms of working memory training: a review. Psychological Research, 78(6), 803-820. https://doi.org/10.1007/s00426-013-0524-6

Wager, T. D., \& Smith, E. E. (2003). Neuroimaging studies of working memory: A metaanalysis. Cognitive, Affective and Behavioral Neuroscience, 3(4), 255-274. Retrieved from http://www.scopus.com/inward/record.url?eid=2-s2.02342597739\&partnerID=40\&md5=d13bba90ed9b65ae16fd931 accf885b1

Wager, T. D., Sylvester, C.-Y. C., Lacey, S. C., Nee, D. E., Franklin, M., \& Jonides, J. (2005). Common and unique components of response inhibition revealed by fMRI. NeuroImage, $27(2)$ $323-340$. https://doi.org/10.1016/J.NEUROIMAGE.2005.01.054

Waldschmidt, J. G., \& Ashby, F. G. (2011). Cortical and striatal contributions to automaticity in information-integration categorization. NeuroImage, 56(3), 17911802. https://doi.org/10.1016/j.neuroimage.2011.02.011

Waris, O., Soveri, A., \& Laine, M. (2015). Transfer after Working Memory Updating Training, 1-19. https://doi.org/10.1371/journal.pone.0138734

Wass, C., Pizzo, A., Sauce, B., Kawasumi, Y., Sturzoiu, T., Ree, F., ... Matzel, L. D. (2013). Dopamine D1 sensitivity in the prefrontal cortex predicts general cognitive abilities and is modulated by working memory training. Learning \& Memory, 20(11), 617-627. https://doi.org/10.1101/lm.031971.113

Watanabe, Y., \& Funahashi, S. (2012). Thalamic mediodorsal nucleus and working memory. Neuroscience and Biobehavioral Reviews, 36(1), 134-142. https://doi.org/10.1016/j.neubiorev.2011.05.003

Waters, S. (1992). The Role of High-Level Speech Planning in Rehearsal : Evidence from Patients with Apraxia of Speech.

Weicker, J., Villringer, A., \& Thöne-Otto, A. (2016). Can impaired working memory functioning be improved by training? A meta-analysis with a special focus on brain injured patients. Neuropsychology, 30(2), 190-212. https://doi.org/10.1037/neu0000227

Westerberg, H., \& Klingberg, T. (2007). Changes in cortical activity after training of working memory - a single-subject analysis. Physiology and Behavior, 92(1-2), 186-192. https://doi.org/10.1016/j.physbeh.2007.05.041

Yamashita, M., Kawato, M., \& Imamizu, H. (2015). Predicting learning plateau of working memory from whole-brain intrinsic network connectivity patterns. Scientific Reports, 5, 7622. https://doi.org/10.1038/srep07622

Yan, C.-G., Cheung, B., Kelly, C., Colcombe, S., Craddock, R. C., Di Martino, A., ... Milham, M. P. (2013). A comprehensive assessment of regional variation in the impact of head micromovements on functional connectomics. NeuroImage , 76, 183201. https://doi.org/10.1016/j.neuroimage.2013.03.004

Yaple, Z., \& Arsalidou, M. (2018). N-back Working Memory Task: Meta-analysis of Normative fMRI Studies With Children. Child Development, $O(0)$. https://doi.org/10.1111/cdev.13080

Yeung, M. S. Y., Zdunek, S., Bergmann, O., Bernard, S., Salehpour, M., Alkass, K., ... Frisén, J. (2014). Dynamics of Oligodendrocyte Generation and Myelination in the Human Brain. Cell, 159(4), 766-774. https://doi.org/10.1016/J.CELL.2014.10.011

Zou, Q., Ross, T. J., Gu, H., Geng, X., Zuo, X.-N., Hong, L. E., ... Yang, Y. (2013). Intrinsic resting-state activity predicts working memory brain activation and behavioral performance. Human Brain Mapping, 34(12), 3204-3215. https://doi.org/10.1002/hbm.22136 
